\title{
Computational Strategies for Faster Combustion Simulations with Detailed Chemistry
}

Jose A. Escobar-Vargas

West Virginia University

Follow this and additional works at: https://researchrepository.wvu.edu/etd

\section{Recommended Citation}

Escobar-Vargas, Jose A., "Computational Strategies for Faster Combustion Simulations with Detailed Chemistry" (2014). Graduate Theses, Dissertations, and Problem Reports. 189.

https://researchrepository.wvu.edu/etd/189

This Dissertation is protected by copyright and/or related rights. It has been brought to you by the The Research Repository @ WVU with permission from the rights-holder(s). You are free to use this Dissertation in any way that is permitted by the copyright and related rights legislation that applies to your use. For other uses you must obtain permission from the rights-holder(s) directly, unless additional rights are indicated by a Creative Commons license in the record and/ or on the work itself. This Dissertation has been accepted for inclusion in WVU Graduate Theses, Dissertations, and Problem Reports collection by an authorized administrator of The Research Repository @ WVU.

For more information, please contact researchrepository@mail.wvu.edu. 


\title{
Computational Strategies for Faster Combustion Simulations with Detailed Chemistry
}

\author{
Jose A. Escobar-Vargas
}

\begin{abstract}
Dissertation submitted
to the Benjamin M. Statler College of Engineering and Mineral Resources at West Virginia University

in partial fulfillment of the requirements for the degree of

Doctor of Philosophy in

Mechanical Engineering
\end{abstract}

Ismail Celik, Ph.D., Chair

V'yacheslav Akkerman, Ph.D.

Christopher Atkinson, Sc.D.

Dady Dadyburjor, Ph.D.

Donald Ferguson, Ph.D.

Gregory Thompson, Ph.D.

Department of Mechanical and Aerospace Engineering

Morgantown, West Virginia

2014

Keywords: CFD, Combustion, Swirl Stabilized Combustion, Chemical Mechanism, Chemical Reactor Network, Stiff Equations

Copyright 2014 Jose A. Escobar-Vargas 


\title{
ABSTRACT \\ Computational Strategies for Faster Combustion Simulations with Detailed Chemistry
}

\author{
Jose A. Escobar-Vargas
}

Combustion of fossil fuels is still the biggest source of power generation in the world. However, pollutants released to the atmosphere from combustion represent a risk for human health and the environment. Hence it is desirable to design a combustor that produces the maximum useful thermal power output while keeping low concentration levels of harmful emissions such as $\mathrm{CO}$, P.M., $\mathrm{NO}_{\mathrm{x}}$, and $\mathrm{SO}_{\mathrm{x}}$. In the past, combustor design was aided by the compilation of large sets of experimental data and the development of empirical correlations which is an expensive process. Nowadays numerical simulations have become an important tool in the research and design of combustors. Numerical simulations allow the study of combustion systems under hazardous conditions and beyond their performance limits, and they are usually inexpensive and fast (compared to experiments). The main bottle-neck in combustion simulations is the accurate prediction of the concentration of the many species involved in combustion. Current computational fluid dynamic (CFD) simulations commonly use simplified versions of the chemical reaction mechanisms. But utilization of simplified chemical models comes with the associated inaccuracy while saving computational time.

In the present study the virtues of the chemical reactor network (CRN) approach are investigated and a new integration method is proposed to accelerate the calculation of species concentrations using reduced and detailed chemical mechanisms. Utilization of the CRN approach enabled the implementation of a detailed methane-air chemical mechanism that incorporates 53 chemical species and 325 reactions. The CRN approach was applied to two combustor configurations: a premixed methane-air swirl burner, and a non-premixed methane-air swirl burner. The CRN was built using results from the CFD simulations that were obtained using simplified chemical mechanisms with just one or two reactions. Numerical predictions of the premixed combustor behavior obtained using CRN simulations were compared with other CFD simulations that used mechanisms with more reactions and chemical species. The CRN results closely matched the CFD simulations with larger chemical mechanisms, the maximum relative difference of the predicted concentration for the major species (i.e. $\mathrm{O}_{2}, \mathrm{CO}_{2}, \mathrm{H}_{2} \mathrm{O}$, and $\mathrm{N}_{2}$ ) was $2.82 \%$ when compared to the CFD simulations. The calculation time of the CRN was greatly reduced, the maximum reduction of the CRN simulation took only one seventh of the computational time when compared with a CFD simulation. The CRN simulations of the non-premixed burner were also compared with experiments. Predicted spatial profiles of velocity, temperature, and mass fraction concentrations were compared with measurements. Results showed that the velocity and some mass fraction profiles matched the experimental measurements near the dump plane but it was found that downstream of the dump plane the temperature was overpredicted. Due to the temperature 
overprediction, the maximum difference was $250[\mathrm{~K}]$, the nitrogen oxide $(\mathrm{NO})$ concentration was overpredicted by 30 [ppm]. The relative difference of the predicted NO at the outlet of the combustor is $150 \%$ when compared with the experimental value.

Further, a novel integration method named log-time integration method (LTIM) was developed to calculate the solution of ideal reactors used in the CRN simulations. The integration method consists of the transformation of the time variable to the logarithmic space along with the use of variable time steps. The LTIM approach was applied to the solution of a perfectly stirred reactor (PSR) using a detailed chemical mechanism. PSR-LTIM results were compared with a commercial PSR code which is available in the CHEMKIN software package. The maximum relatively difference of the concentration of the species of interest was only $1 \%$. Calculated species concentration using the PSR-LTIM matched the results from CHEMKIN with comparable computational time, the computational time of the PSR-LTIM was 5.3 [s] and for CHEMKIN was 3 [s]. The integration method was compared to higher order integration methods available in the literature producing satisfactory results with less CPU time, the LTIM approach took one fifth of the computational time of a higher order integration method. The LTIM was also applied to the solution of a premixed one dimensional methane-air flame, FLAME-LTIM, where a mechanism incorporating nine chemical species and five global reactions mechanism was used. Calculated temperature and mass fraction profiles matched closely the results obtained using the equivalent commercial code CHEMKIN PREMIX. The relative temperature difference at the outlet of the domain was $0.5 \%$ and the maximum difference in the chemical specie concentration at the outlet of the domain was $13.2 \%$.

The outcome of the present research can be used to perform a rapid design analysis of gas turbines and similar combustors to achieve low levels of emissions.

Keywords: CFD, Combustion, Swirl Stabilized Combustion, Chemical Mechanism, Chemical Reactor Network, Stiff Equations. 
Dedicated to my mother

\section{María del Rosario Vargas Castillo $\dagger$}




\section{Acknowledgements}

I would like to sincerely thank my advisor, Dr. Ismail Celik for the opportunity to work with him and for his guidance and encouragement through this study. I really appreciate all his contributions, ideas, and founding to make my Ph.D. a productive and stimulating endeavor. His knowledge and hardworking philosophy are qualities that I aspire to achieve. Special thanks are to the members in my committee: Dr. V'yacheslav Akkerman, Dr. Christopher Atkinson, Dr. Dady Dadyburjor, Dr. Donald Ferguson, and Dr. Gregory Thompson for taking time from their busy schedule to review my work and for their useful comments. I am also grateful to the United States Department of Energy (USDOE) through the National Energy Technology Laboratory (NETL) that provided the founding for this work. I would also like to thank my former advisor, Dr. Abel Hernandez-Guerrero for his guidance through my undergraduate studies in Mexico and for his invaluable help with the application process for graduate school in the United States of America.

Also, I would like to thank my CFD Lab friends; Raju Pakalapati, Jaggu Nanduri, Alejandro Posada, Francisco Elizalde, Francisco Pino, Ertan Karaismail, Nihan Cayan, Sergio Escobar, Albio Gutierrez, Hayri Sezer, Sharad Ravinuthala, Orlando Ugarte, and Omar Meza for their friendship and advice during my Ph.D. Special thanks to Dr. Raju Pakalapati for his support and useful answers he kindly provided to my never ending questions.

Very special thanks to my parents Elias Escobar and Maria del Rosario Vargas and to my brothers and sisters; Leticia, Sergio, Araceli, David, Linda, and Alondra for their encouragement and the faith they put on me. Without your support this would have not been possible. 


\section{Table of Contents}

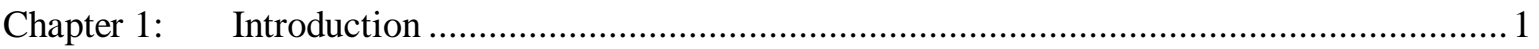

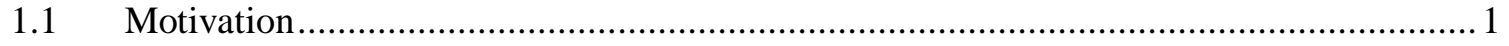

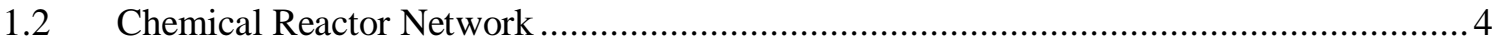

1.3 Log-Time Integration Method (LTIM) ..................................................................

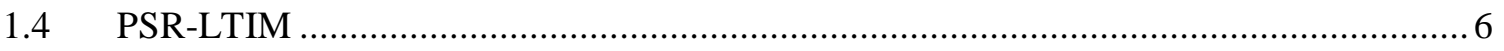

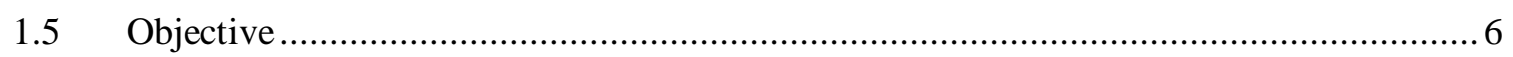

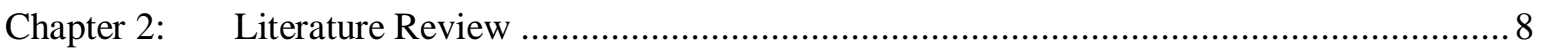

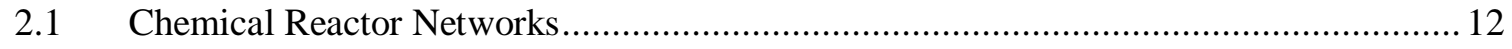

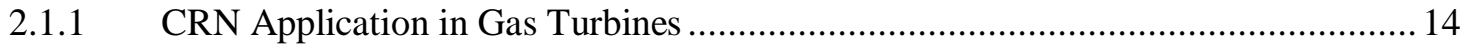

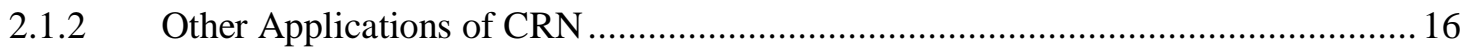

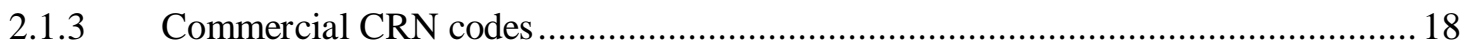

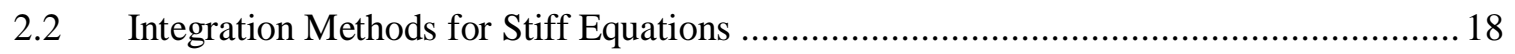

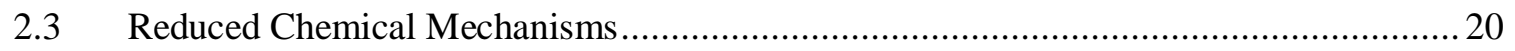

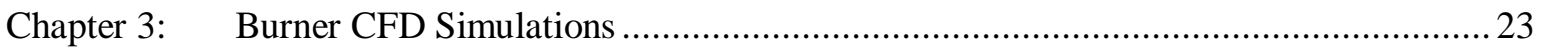

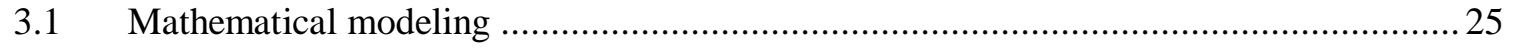

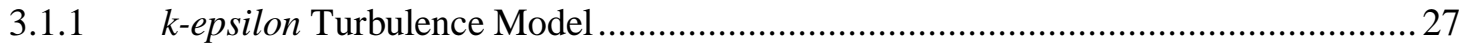

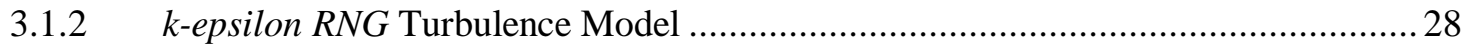

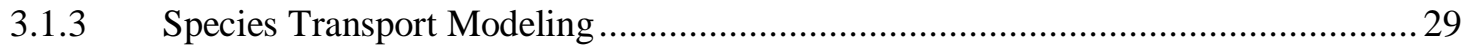

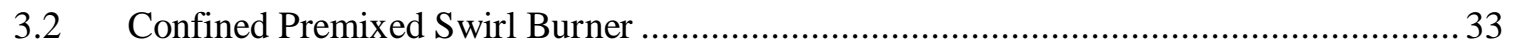

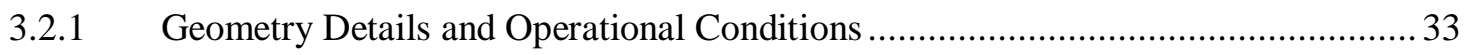

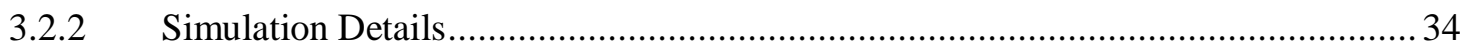

3.2.3 CFD Results for Non-Reacting Flow - NETL Burner ....................................... 37

3.2.4 CFD Results for Reacting Flow - NETL Burner............................................ 41

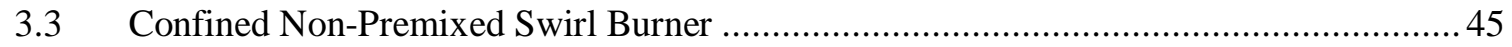

3.3.1 Geometry Details and Operational Conditions …........................................... 45

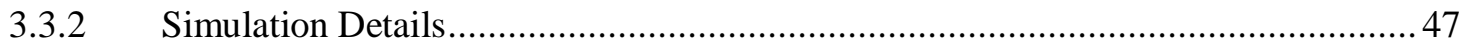

3.3.3 CFD Results for Reacting Flow - TECFLAM Burner......................................... 49

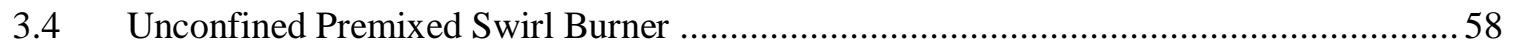

3.4.1 Geometry Details and Operational Conditions ...............................................5

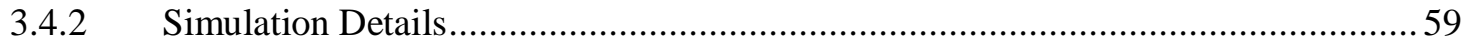

3.4.3 CFD Results - Unconfined Premixed Swirl Burner ............................................ 66 


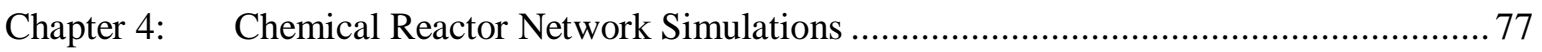

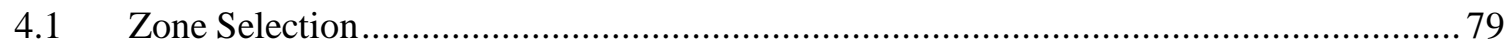

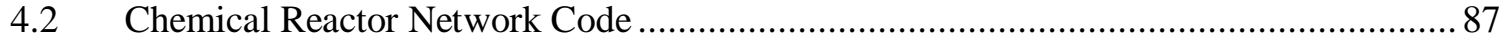

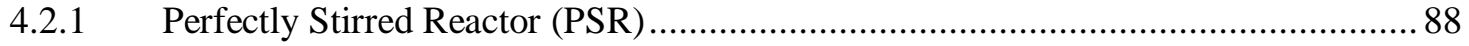

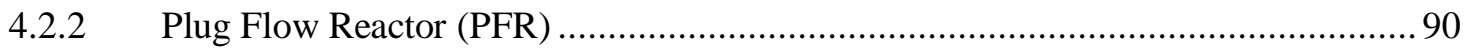

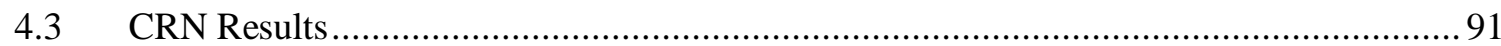

4.3.1 Domain Decomposition - NETL Burner ....................................................... 91

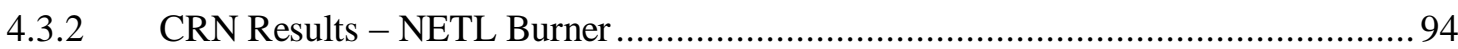

4.3.3 Domain Decomposition - TECFLAM Burner ................................................ 100

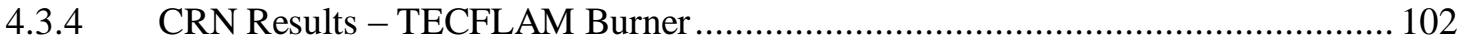

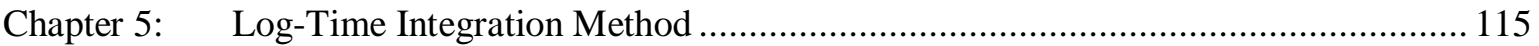

5.1 Zero Dimensional Transient Model Applications …............................................... 115

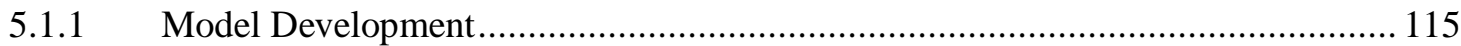

5.1.2 Zero Dimensional Transient Model - NO System............................................ 117

5.1.3 Zero Dimensional Transient Model - Methane-Air System .................................. 120

5.1.4 Zero Dimensional Transient Model - Propane-Air System .............................. 121

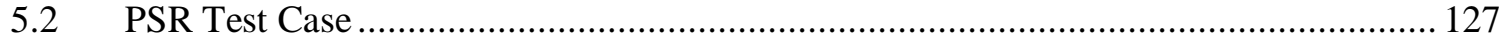

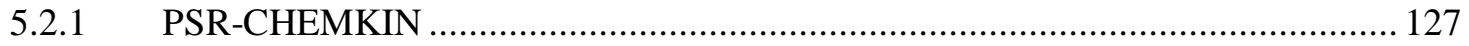

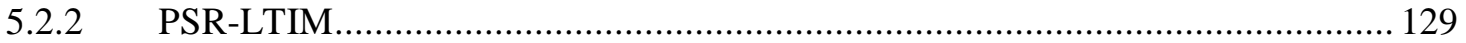

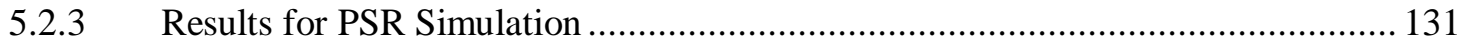

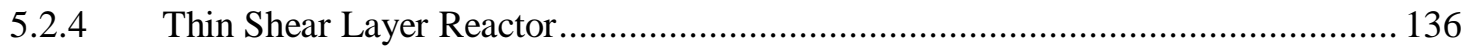

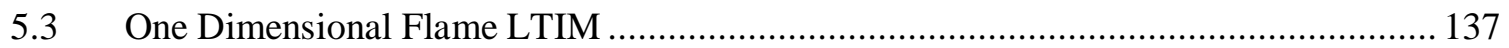

5.3.1 One Dimensional Flame LTIM Results at Fixed Temperature............................. 140

5.3.2 One Dimensional Flame LTIM Results at Variable Temperature. ........................ 142

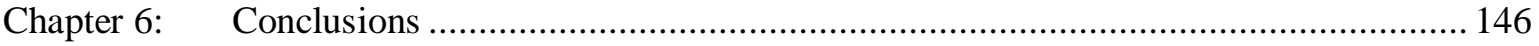

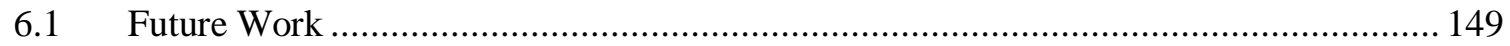

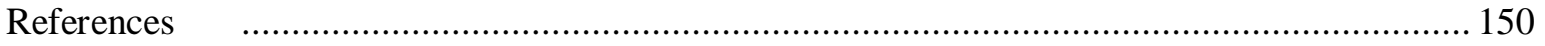

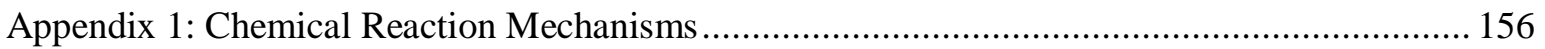

Appendix 2: Fifth Order Runge-Kutta Integration Method....................................................... 160

Appendix 3: k-epsilon RNG and RSM Turbulence Model Comparison................................... 162 


\section{List of Figures}

Figure 2.1. Dependence of the Number of Reactions and the Statistical Computational Cost in Commonly used Chemical Mechanisms (Lu, Law and Yoo, et al. 2009). 11

Figure 2.2. Zone Modeling of a Gas-Turbine Can Type Combustor (Swithenbank, Poll and Vincent 1973) .13

Figure 2.3. Zone Modeling of ALF-502 Gas Combustor (Rubins and Pratt 1991). 14

Figure 2.4. CRN Layout of a) Diffusion Combustor, and b) Premixed Combustor (Andreini and Facchini 2004). 15

Figure 2.5. Zone Modeling of the Single-Injector, Can-Type, GT Combustor (Novosselov 2006).15

Figure 2.6. a) Geometry of the Combustor, b) CRN Obtained by (Kanniche 2010). 16

Figure 2.7. a) Furnace Sketch, b) CRN developed by Falcitelli et al. (Falcitelli, Pasini and Tognotti 2002). 17

Figure 2.8. CRN Layout Created using Energico Software. 18

Figure 3.1. Swirl Burner Inlet Configurations. a) Annular Vaned Swirler, b) Tangential Flow Injection (Valera-Medina, et al. 2011). 24

Figure 3.2. Characteristic Flow Field of a Confined Swirl Flame (Meier, et al. 2000) 24

Figure 3.3. NETL Burner Sketch. 33

Figure 3.4. CFD Simulations of a) Three Dimensional Inlet Section used for Non-Reacting Flow Simulations and b) Two Dimensional Axisymmetric Combustor Section used for the Reacting Flow Simulation. 35 
Figure 3.5. NETL Burner Boundary Conditions

Figure 3.6. Grid Independence of Axial Velocity at Dump Plane, theta $=0$ [deg] .38

Figure 3.7. PIV Contour of Cold Flow Axial Velocity [m/s], theta $=0[\mathrm{deg}]($ Strakey 2010). 39

Figure 3.8. Comparison of the PIV Measurements and the Predicted Non-Reacting Flow Velocity Profiles a) Axial Velocity, b) Radial Velocity, and c) Tangential Velocity. .39

Figure 3.9. Averaged Velocity Profiles $[\mathrm{m} / \mathrm{s}]$ along Theta Direction, a) Axial Velocity, b) Radial Velocity, and c) Tangential Velocity. 41

Figure 3.10. Axial Velocity Contours $[\mathrm{m} / \mathrm{s}]$ from Three Dimensional Non-Reacting Flow Simulation at; a) 25 [mm] Upstream of the Dump Plane, b) Dump Plane. .41

Figure 3.11. Axial Velocity Contour [m/s] with Streamlines from Two Dimensional Axisymmetric Reacting Flow Simulations. One Step Global Mechanism. .42

Figure 3.12. Two Dimensional Axisymmetric RANS Simulations. a) Temperature Contours One Step Global Mechanism, b) Outlet Temperature Profile using Different Chemical Mechanisms.... 43

Figure 3.13. Outlet Mole Fraction Profile using Different Chemical Mechanisms. a) Water, b) Oxygen. 44

Figure 3.14. Schematic of the TECFLAM Burner (Meier, et al. 2000). 46

Figure 3.15. a) Sketch of the TECFLAM Burner, b) TECFLAM Computational Domain. 47

Figure 3.16. Measured Velocity Profiles at the Inlet of the TECFLAM Burner (Landenfeld, et al. 1998). a) Axial, b) Radial, and c) Tangential Velocity Components. 48 
Figure 3.17. TECFLAM Burner Simulation Contours, a) Axial Velocity and Streamlines, b)

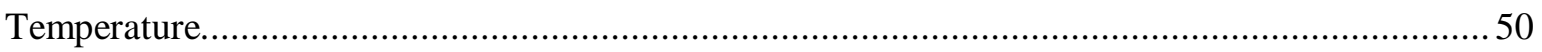

Figure 3.18. Axial Velocity Profiles. Circles - Experiments, Dash line - CFD Simulations..........51

Figure 3.19. Tangential Velocity Profiles. Diamonds - Experiments, Dash line - CFD Simulations.

Figure 3.20. Radial Velocity Profiles. Triangles - Experiments, Dash line - CFD Simulations. ... 52

Figure 3.21. Temperature Profiles. Left Triangles - Experiments, Dash line - CFD Simulations.. 54

Figure 3.22. Methane Mass Fraction Profiles. Diamonds - Experiments, Dash line - CFD

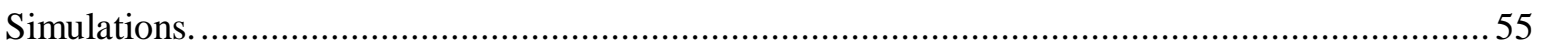

Figure 3.23. Oxygen Mass Fraction Profiles. Diamonds - Experiments, Dash line - CFD

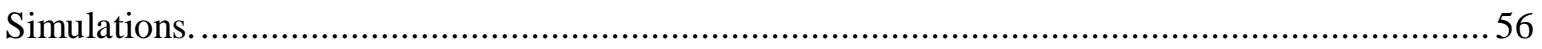

Figure 3.24. Carbon Dioxide Mass Fraction Profiles. Diamonds - Experiments, Dash line - CFD Simulations.

Figure 3.25. Water Vapor Mass Fraction Profiles. Diamonds - Experiments, Dash line - CFD Simulations.

Figure 3.26. Experimental Lab-Scale Swirl Stabilized Burner (Kim, Arghode and Gupta 2009)...59

Figure 3.27. Two Dimensional Sketch of the Unconfined Swirl Stabilized Burner (Gupta and Khalil 2013). 60

Figure 3.28. Computational Domains of the Unconfined Swirl Stabilized Burner. 61

Figure 3.29. Boundary Conditions for the Unconfined Swirl Stabilized Burner. 63 
Figure 3.30. a) Swirler, b) Computational Domain of the Inlet.

Figure 3.31. Non-Uniform Velocity Profiles. a) Scaled from NETL Burner, b) Scaled from Chigier and Beer (Chigier and Beer 1964), c) Experiments (Gupta and Khalil 2013).... .65

Figure 3.32. Axial Velocity $[\mathrm{m} / \mathrm{s}]$ Contours Non-Reacting Flow. a) Two Dimensional Axisymmetric with NETL Scaled Velocity Profiles, b) Experiments (Gupta and Khalil 2013)......67

Figure 3.33. Reacting Flow with Uniform Velocity. a) Temperature Contours $\left[{ }^{\circ} \mathrm{C}\right]$, b) Axial Velocity $[\mathrm{m} / \mathrm{s}]$ Contours and Streamlines. 68

Figure 3.34. Reacting Flow with Uniform Velocity Profiles. a) Temperature Profiles $\left[{ }^{\circ} \mathrm{C}\right]$, b) $\%$ Vol Oxygen, c) \% Vol Carbon Dioxide, d) Nitrogen Oxide [ppm].

Figure 3.35. Reacting Flow with Uniform Axial Velocity [m/s] Contours. a) CFD Simulations, b)

Experiments. .70

Figure 3.36. Reacting Flow Temperature Profiles. a) Uniform Velocity Profiles, b) Non-Uniform Velocity Profiles Scaled from Chigier and Beer (Chigier and Beer 1964), c) Non-Uniform Velocity from Gupta and Khalil (Gupta and Khalil 2013), d) Three Dimensional Domain using Chigier and Beer Velocity Profiles, e) Three Dimensional Domain with Swirler. .72

Figure 3.37. Reacting Flow Axial Velocity [m/s] Contours. a) Experiments, b) Uniform Velocity Profiles, c) Non-Uniform Velocity Profiles Scaled from Chigier and Beer (Chigier and Beer 1964), d) Non-Uniform Velocity from Gupta and Khalil (Gupta and Khalil 2013), e) Three Dimensional Domain using Chigier and Beer Velocity Profiles, f) Three Dimensional Domain with Swirler.....75

Figure 4.1. Mesh Reconstruction using MATLAB. 79

Figure 4.2. Zone Division of the CFD Domain. a) Complete Domain, b) Flame Region. 82 
Figure 4.3. Cell Neighbors.

Figure 4.4. Zone Boundaries. a) Complete Domain, b) Flame Region. 84

Figure 4.5. Example of a CRN Layout. 86

Figure 4.6. Flow Diagram of the CRN Computer Program. 87

Figure 4.7. Perfectly Stirred Reactor (PSR) concept. 89

Figure 4.8. PFR Driver Flow Diagram. .90

Figure 4.9. PFR Approximation Using Series of PSRs (Levenspiel 1962). .91

Figure 4.10. Confined Premixed Burner Predictions. a) Temperature [K] Contours, b) Mass Fraction of Carbon Dioxide Contours, c) Axial Velocity [m/s] Contours and Streamlines. .92

Figure 4.11. ID Cell Contours of the NETL Burner using CFD with Global Mechanism Data. a) Complete Domain, b) Flame Region. .93

Figure 4.12. Outlet Mole Fractions. CRN GRI 3.0, CFD ARM9. .94

Figure 4.13. Outlet Mole Fractions. CRN GRI 3.0, CFD ARM19. .96

Figure 4.14. Parallel Speedup Calculation Using Amdhal's Law.

Figure 4.15. Serial CPU Time. .99

Figure 4.16. CFD Solution Contours TECFLAM Burner. a) Temperature [K] Contours, b) Water Mass Fraction Contours, c) Axial Velocity [m/s] Contours and Streamlines. 101

Figure 4.17. ID Cell Contours TECFLAM Burner. a) $n_{\text {Temp }}=9, n_{H 2 O}=9$, b) $n_{\text {Temp }}=18, n_{H 2 O}=18$, c) $n_{\text {Temp }}=36, n_{H 2 O}=36$. 102 
Figure 4.18. Contour Reconstruction using Results from CRN, $n_{\text {Temp }}=18$ and $n_{H 2 O}=18$. a) Temperature $[\mathrm{K}], \mathrm{b})$ Water Mass Fraction. 103

Figure 4.19. Temperature Profiles. Left Triangles - Experiments, Dash line - CFD Simulations with Two Step Global Mechanism, Blue Circles - CRN Simulations 104

Figure 4.20. Methane Mass Fraction Profiles. Red Diamonds - Experiments, Dash line - CFD Simulations with Two Step Global Mechanism, Blue Circles - CRN Simulations. 105

Figure 4.21. Oxygen Mass Fraction Profiles. Red Diamonds - Experiments, Dash line - CFD Simulations with Two Step Global Mechanism, Blue Circles - CRN Simulations. 106

Figure 4.22. Carbon Dioxide Mass Fraction Profiles. Red Diamonds - Experiments, Dash line CFD Simulations with Two Step Global Mechanism, Blue Circles - CRN Simulations. 107

Figure 4.23. Water Mass Fraction Profiles. Red Diamonds - Experiments, Dash line - CFD Simulations with Two Step Global Mechanism, Blue Circles - CRN Simulations. 108

Figure 4.24. Nitrogen Oxide Mass Fraction Profiles. Blue Circles - CRN Simulations. 109

Figure 4.25. CRN Predicted NO Concentration TECFLAM Burner. 111

Figure 4.26. CRN CPU Time TECFLAM Burner. 113

Figure 5.1. a) Equation 5.5 Variation with Time [s] , b) Equation 5.5 Variation along Logarithmic Time Space. 117

Figure 5.2. Transient Solution of Reaction 5.6. a) LTIM, b) R.K. $5^{\text {th }}$ Order Method. 119

Figure 5.3. Transient Mass Fraction of Propane-Air Mechanism, $t_{\max }=1 \times 10^{-4}[\mathrm{~s}]$. a) Explicit Euler Constant $d t$, b) Explicit Euler Variable $d t$, c) LTIM. 125 
Figure 5.4. Transients of Carbon Monoxide and Nitrogen Oxide Mass Fractions from the PSR-

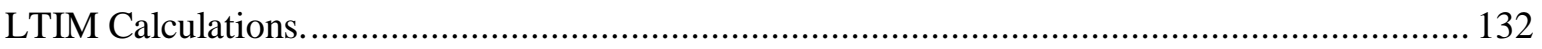

Figure 5.5. Calculated CPU Time of the PSR Calculations. ................................................ 135

Figure 5.6. One Dimensional Flame Program Flowchart................................................... 140

Figure 5.7. One Dimensional Flame LTIM Temperature Profile (Fixed). ............................... 141

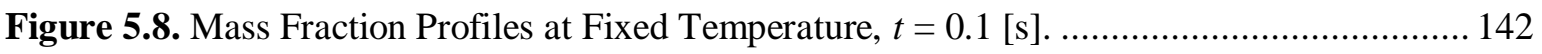

Figure 5.9. Temperature Profile Prediction. ....................................................................... 143

Figure 5.10. Species Mass Fraction Comparison............................................................ 144

Figure A.3.1. Temperature Profile Comparison, a) k-epsilon $R N G$, b) $R S M$ Turbulence Model. . 163

Figure A.3.2. \% Vol Carbon Dioxide Profile Comparison, a) k-epsilon $R N G$, b) $R S M$ Turbulence

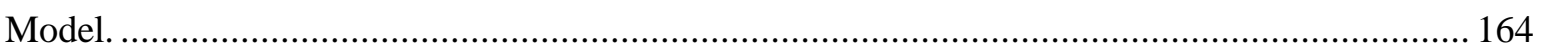

Figure A.3.3. \% Vol Oxygen Profile Comparison, a) k-epsilon RNG, b) RSM Turbulence Model. 164 


\section{List of Tables}

Table 3.1. Confined Premixed Swirl Burner Simulation Details. 35

Table 3.2. TECFLAM Geometry and Operational Conditions. 46

Table 3.3. Confined Non-Premixed Swirl Burner Simulation Details. 48

Table 3.4. Unconfined Premixed Swirl Burner Simulation Details. 66

Table 4.1. Mole Fraction Comparison. CFD ARM9, CRN GRI 3.0. 95

Table 4.2. CRN Extra Species. CFD ARM9, CRN GRI 3.0..... 95

Table 4.3. Mole Fractions Comparison. CFD ARM19, CRN GRI 3.0. 96

Table 4.4. Calculation Time for Case 1 NETL Burner. 97

Table 4.5. CRN Cases for TECFLAM Burner. 110

Table 5.1. ARM9 Mass Fractions. 120

Table 5.2. Summary of the Modified Eight-Step Kinetic Model. 121

Table 5.3. Propane Air Mechanism Summary. 126

Table 5.4. PSR Conditions. 131

Table 5.5. PSR-LTIM Predicted Mole Fractions of the Species of Interest. 133

Table 5.6. PSR-LTIM Predicted Mole Fractions Continued. 133

Table 5.7. Flame Speed Calculation for an Equivalence Ratio $=0.9$, and $P=1[\mathrm{~atm}]$. 145 
Table A.3.1. Effect of the Turbulence Model - Simulation Details. 


\section{Chapter 1: Introduction}

\subsection{Motivation}

Combustion of fossil fuels remains as the largest source of power generation in the U.S in 2014. However, pollutants released to the atmosphere from combustion units represent a risk for human health and the environment. In order to reduce the negative impact of fossil fuel combustion units the federal government has imposed controls for the regulated pollutants such as; $\mathrm{CO}, \mathrm{NO}_{\mathrm{x}}$, $\mathrm{O}_{3}, \mathrm{~Pb}$, particulate matter $(\mathrm{PM}), \mathrm{SO}_{\mathrm{x}}$. Environmental controls have led research to find innovative strategies to reduce the negative impact of combustion in the environment. In the past, combustion design was aided by the compilation of large sets of experimental data and the development of empirical correlations which made the design of new combustors an expensive enterprise. Nowadays numerical simulations have become an important tool in research and design of combustion systems. The main advantages of numerical simulations are: relatively low cost of development, speed (compared to experiments), and relative ease with which parametric studies can be conducted. Despite all the aforementioned advantages, simulation of combustion phenomena is far from accurate and detailed predictions. The difficulty of combustion simulation is due to the many physical processes that occur simultaneously such as turbulent fluid dynamics, chemical reactions, heat and mass transfer.

In order to accurately predict the combustion phenomena it is necessary to resolve essentially all characteristic temporal and spatial scales. The Direct Numerical Simulation (DNS) approach solves all scales in the flow but is computationally expensive. To illustrate the dimension of the problem, a three dimensional computer simulation of a non-premixed $\mathrm{CO} / \mathrm{H}_{2} / \mathrm{N}_{2}$-air jet flame reported by Hawkes (Hawkes, et al. 2005) with 12 species and 33 reactions used a computational mesh with 40 million cells. The calculation ran on 480 processors on the MPP2 HP-Itanium-2 cluster at the Pacific Northwest National Laboratory (PNNL). Approximately 50 days were 
required to complete the simulation. Despite the progress in parallel computing in the past decades, DNS of reacting systems is confined to cases with low turbulent Reynolds numbers $\left(\sim \operatorname{Re}<10^{4}\right.$ (Versteeg and Malalasekera 2007)) and chemically simple fuels due to the spatial and temporal restrictions. DNS of practical combustion burners is impossible with current computational tools.

The combustion research community has long used simplified models to study combustors for practical applications. Such models are mainly simplifications of the turbulence phenomena and the chemical mechanisms; these models will be discussed in the following chapters. However, detailed chemical mechanisms are necessary to study the consumption or production of the species involved in combustion phenomena to keep modeling errors to a minimum. Chemical mechanisms for common fuels contain a large number of chemical species and reactions (e.g. $\mathrm{CH}_{4}$ detailed mechanism contains 53 species and 325 reactions (Smith, et al. 1999)) making computer simulations virtually impossible due the excessive amount of Central Processing Unit (CPU) time needed to calculate the solution. The required amount of CPU time is large because of the following reasons:

1. Number of species. Determining the concentration of each one of the chemical species requires the solution of a partial differential equation.

2. Time scales. Combustion phenomena are characterized by having a wide range of time scales. Some species have a very small characteristic time scale (e.g. $\sim 10^{-6}-10^{-8}[\mathrm{~s}]$ ) while others have long time scales $\left(\sim 10^{-1}[\mathrm{~s}]\right)$ making the system of equations stiff and the solution very time consuming. The time scale of the chemical species is defined as $\tau_{i}=\left(Y_{i, \text { initial }} /\left(\dot{\omega}_{i} * M W_{i} / \rho\right)\right)^{-1}$. Where $Y_{i, \text { initial }}$ is the initial mass fraction of the specie $i$ [dimensionless], $\dot{\omega}_{i}$ is the net reaction rate of specie $i\left[\mathrm{~mol} /\left(\mathrm{cm}^{3} * \mathrm{~s}\right)\right], M W_{i}$ is the molecular weight of specie $i[\mathrm{~g} / \mathrm{mol}], \rho$ is the density of the mixture $\left[\left(\mathrm{g} / \mathrm{cm}^{3}\right)\right]$. 
3. Low pollutant concentration. Concentration of $\mathrm{NO}_{\mathrm{x}}$ for example is limited to tens of parts per million in volume (ppmv) in typical combustors which means that any assumption made in the model could have significant effect on the species at these very low concentrations.

The most restrictive of the points mentioned above is related to the time scales (stiff systems). Current numerical integration methods for stiff equations are computationally intensive and complex to program. A substantial effort has been put into resolving this issue in the past three decades. Two main areas can be mentioned:

I. Explicit integrators: This kind of integrator is accurate but requires the time step $d t$ to be smaller than the smallest characteristic time scale of the system otherwise the numerical prediction will diverge from the true solution. These integrators do not require internal iterations at every time step and most of them are self-starting.

II. Implicit integrators: Here relatively large time steps can be used. The drawback is that it is necessary to calculate the Jacobian matrix. The size of the matrix is proportional to the total number of species in the system and it has to be often recalculated. The requirement for calculating the Jacobian inversion makes this option impractical for systems with large number of species. Most of the implicit integrators require a good initial guess otherwise the solution will diverge from the true solution.

Another way to reduce the complexity of the problem is to reduce the size of the detailed chemical mechanisms using assumptions such as the quasi-steady state assumption (QSSA) and partial equilibrium (PE). These assumptions allow a reduction in the number of species considered for combustion simulations. This approach produces good results for species whose concentration is relatively high, however results are not as accurate for the so-called trace species. Trace species are, chemical species at a relatively low concentration and their actual concentration could be very 
different than its PE or QSSA concentration. Since prediction of the pollutant concentration is one of the main goals in combustion simulation, accurate calculation of trace species is necessary because some critical pollutants (e.g. $\mathrm{NO}_{\mathrm{x}}$ ) can be catalogued as trace species.

The present study proposes the utilization of the following tools to predict detailed chemistry composition in the shortest time as possible:

I. Chemical reactor network (CRN).

II. Log-time integration method (LTIM).

III. Perfectly stirred reactors (PSR) used with LTIM.

In what follows each of the topics are elaborated upon in detail.

\subsection{Chemical Reactor Network}

In this study it is proposed to use the chemical reactor network (CRN) concept in order to predict the emissions of methane-air burners using detailed chemical mechanisms. Utilization of detailed mechanisms is important to accurately predict the concentration of trace pollutants. Assumptions taken to reduce the size of detailed mechanism could greatly impact the prediction of species of interest. The CRN approach ( (Novosselov 2006), (Frassoldati, et al. 2005)) used in this study is described below.

First, computational fluid dynamics (CFD) simulations of the combustor are performed using a commercial CFD code (ANSYS-FLUENT). CFD simulations are performed using relatively computationally inexpensive models. For instance, turbulence modeling is simulated using RANS (Reynolds-Averaged Navier-Stokes) models and chemistry modeling is performed using reduced chemical mechanisms including global reaction mechanisms. 
Second, an in-house computer program is used to create the reactor network layout using the predictions from the CFD simulations as input. The program clusters computational cells into zones called reactors. The clustering depends mainly on the temperature field, the flow field and the concentration of a chemical specie of interest. The criterion to create these zones is that computational cells that share similar characteristics (e.g. temperature, oxygen mass fraction, water mass fraction, etc.) are clustered as one reactor. The computer program also defines the connectivity of these reactors using the mass flux between these zones. The result is a reactor network in which every reactor can be modeled as a simplified element for example a Perfectly Stirred Reactor (PSR) is a zero dimensional unit, or as a Plug Flow Reactor (PFR) which is a one dimensional unit.

The last step in the CRN approach is to model every reactor using a detailed chemical mechanism. Here a separate computer program reads the information from the zone clustering and performs the simulation for each one of the reactors using a detailed chemical mechanism. Simulations were performed using an iterative procedure and the results were compared with experiments or CFD simulations that used reduced mechanisms with more chemical species.

\subsection{Log-Time Integration Method (LTIM)}

An explicit, second order integration method is proposed for the integration of stiff equations. Stiff equations are characterized by large gradients $(\Delta \varphi / \Delta t, \varphi$ being any variable) preceded or followed by a region with small gradient. This novel method was developed by transforming the time variable into the logarithmic space. The transformation of the time variable into the logarithmic space can decrease the steepness in the gradients thus making the integration more tractable. Being an explicit scheme, this method is easy to implement in problems with a large number of species without the requirement of inverting any Jacobian matrix. The second order 
accuracy of the proposed method is achieved by the combination of the logarithmic transformation with the utilization of the modified Euler scheme.

The LTIM performance was compared against traditional integration methods such as the Explicit Euler and the fifth order Runge-Kutta method. This novel method is also implemented in the solution of a one dimensional methane-air laminar flame. Laminar flame speeds and mass fraction profiles were reported and compared against a commercial software, CHEMKIN PREMIX (Kee, et al. 1985).

\subsection{PSR-LTIM}

Finally, the LTIM was integrated into the key element of the CRN which is the PSR. The goal was to develop a fast computational method that can be applied to predict the composition of a mixture in a PSR. Predicted mixture composition using the PSR-LTIM element matched the values calculated using PSR-CHEMKIN (Glarborg, et al. 1986), and the computational time needed to calculate the solution is in the same order of magnitude. The PSR-LTIM represents an option that can be used to solve stiff systems of differential equations instead of the commercial PSRCHEMKIN computer code.

\subsection{Objective}

The goal of this study is to reduce the computational time of combustion simulations with detailed chemistry. The stated objective will be achieved by the implementation of the following steps:

1. Implementation of the chemical reactor network (CRN) concept in order to predict species concentrations using detailed chemical mechanisms.

2. Development of a new time integration method in order to reduce the time needed to solve stiff equation systems. 
3. Implementation of the integration method in idealized reactors; zero dimensional transient reactor, PSR, and one dimensional flame problem.

The outlined strategy will allow the prediction of the composition of a system in less time than a CFD simulation using reduced chemical mechanisms. The implementation of the CRN reduces the complexity (i.e. going from a three dimensional problem to a zero dimensional transient reactor), this reduction allows the implementation of detailed chemistry. The log-time integration method (LTIM) enables further simplicity and robustness without a need for commercial stiff system solvers.

The concepts mentioned above will be put into historical context in the literature review section, the implementation details will be discussed in the methodology section and finally results, discussion of the results, and conclusions will follow. 


\section{Chapter 2: $\quad$ Literature Review}

Combustion modeling of industrial burners is a challenging problem due to the physical phenomena that simultaneously occur during the combustion process, e.g. turbulent fluid dynamics, chemical reactions, heat and mass transfer. The coupling of the aforementioned phenomena produces a non-linear system which makes it extremely difficult to predict. Due to the complexity of the problem, the combustor design has evolved as a product of empirical cut and try process. Over many years, combustor design was aided by the compilation of large sets of experimental data and the development of empirical correlations. Odgers (Odgers 1975) presents the results of a questionnaire completed by combustion designers. Their response indicates a wide variety of design approaches and considerably lack of confidence in combustion theory.

The ability to design new combustors has improved in the past decades mainly because of the utilization of computers. With the increase of computer capabilities the simultaneous solution of all physical phenomena occurring in the burner was envisioned. However, this has not been achieved because of the wide range of spatial and temporal scales that are inherent to combustion phenomena and the large amount of computational cells needed to correctly solve the large number of differential equations involved.

Solution of the flow field is a complex task because the vast majority of modern combustors for industrial applications work under turbulent flow conditions. Turbulent flows are characterized by a velocity field which varies significantly and almost randomly both in space and time. Numerical solution of turbulent flows can be obtained by using the following approaches; Direct Numerical Simulation (DNS), Large Eddy Simulation (LES), and Reynolds Average NavierStokes (RANS) simulations. DNS consists in modeling of all length scales contained in the turbulent flow, in order to capture all scales it is necessary to use a large number of computational cells (proportional to the turbulent Reynolds and the Damköhler numbers) and time steps. Gicquel 
et al. (Gicquel, Staffelbach and Poinsot 2012) estimated the size of computational domain and the length of time the simulation has to run in order to resolve all the scales of the problem. According to Gicquel it is necessary to have $N$ grid points in each direction of the computational domain that must satisfy the following relationship; $\operatorname{Re}_{t} D a<\left(\frac{N}{Q}\right)^{2}$ for simulations using a reaction mechanism with one irreversible reaction. Here the turbulent Reynolds number is defined as $\operatorname{Re}_{t}=\frac{u^{\prime} l_{t}}{v}, u^{\prime}$ being the magnitude of the fluctuating part of the velocity, $l_{t}$ the characteristic turbulent length scale, and $v$ is the kinematic fluid viscosity. The Damköhler number is equal to the characteristic turbulent time scale divided by the characteristic chemical time scale $D a=\frac{\tau_{t}}{\tau_{c}}$. Finally $Q$ is the number of grid points in the thin reaction zone (of the order of 20 for simple chemical schemes). Besides resolving the characteristic time scales it is needed to resolve the temporal behavior of the problem. To properly calculate the time statistics of the problem it is necessary to perform at least $\mathrm{Re}_{t}^{3}<M$ ( $M$ number of time steps) time integrations. Due to the aforementioned conditions, DNS is still limited to simple small geometries and relatively simple fuels. DNS for three-dimensional flows with complex geometries, high turbulent Reynolds numbers and burning large molecule fuels which are usually found in industrial applications are out of today's present computer capabilities.

Another numerical simulation approach is large eddy simulation (LES). In LES the dynamics of the larger-scale flow structures (which are affected by the flow geometry and are not universal) are computed explicitly, while the influence of smaller scales (which have a universal character) is included by relatively simple models. In computational expense, LES lies between DNS and RANS. Nowadays combustion research is trending towards LES to predict burner 
performance. However, LES consumes a significant amount of computational time and most of the relations to model the small turbulent scales are still under development.

Finally, another approach which is commonly used for the prediction of turbulent flows is the Reynolds Average Navier-Stokes (RANS) simulations. RANS involves the solution of the averaged Navier-Stokes equations. The averaged velocity field is affected by the fluctuating velocities represented by extra terms that appear after the averaging of the original governing equations. The extra terms are known as the Reynolds stresses, and these terms are usually modeled using the Boussinesq approximation that states that the contribution of the momentum transfer from the turbulent eddies can be modeled using an eddy viscosity concept. The way the eddy viscosity is calculated depends on the selected turbulence model. Turbulent models vary in complexity from zero equation models (Prandtl mixing length) to up to seven extra equations (three dimensional Reynolds Stress Model (RSM)). The more detailed the turbulence model, the more computationally intensive it becomes, but usually the RANS approach is the least time consuming when compared to DNS and LES approaches.

Although turbulent flow prediction is a significant time consuming process in combustion simulations, the main bottle neck is due to the modeling of the chemical kinetics. Gaseous chemical reactions are modeled using the Arrhenius reaction rate model. The Arrhenius equation gives the rate for each reaction that is considered in the combustion mechanism as

$k=A * T^{\beta} * \exp \left(-\frac{E_{a c t}}{R T}\right)$

Equation 2.1 shows the non-linear dependence of the reaction rate with the temperature, $T$, and activation energy, $E_{a c t}$. The activation energy dictates which reactions proceed faster and which 
slower. As a result, it is common to have a system of equations with a wide range of time scales $\left(\sim 10^{-9}-\sim 10^{0}[\mathrm{~s}]\right)$, this causes stiffness in the equations.

Every reaction included in the simulation has a forward and reverse reaction rate. The collection of reactions that describe the consumption or production of the chemical species is called the chemical mechanism. A chemical mechanism could be as simple as a single reaction global mechanism to a detailed description of the chemistry phenomena with several thousand reactions as can be seen in Figure 2.1.

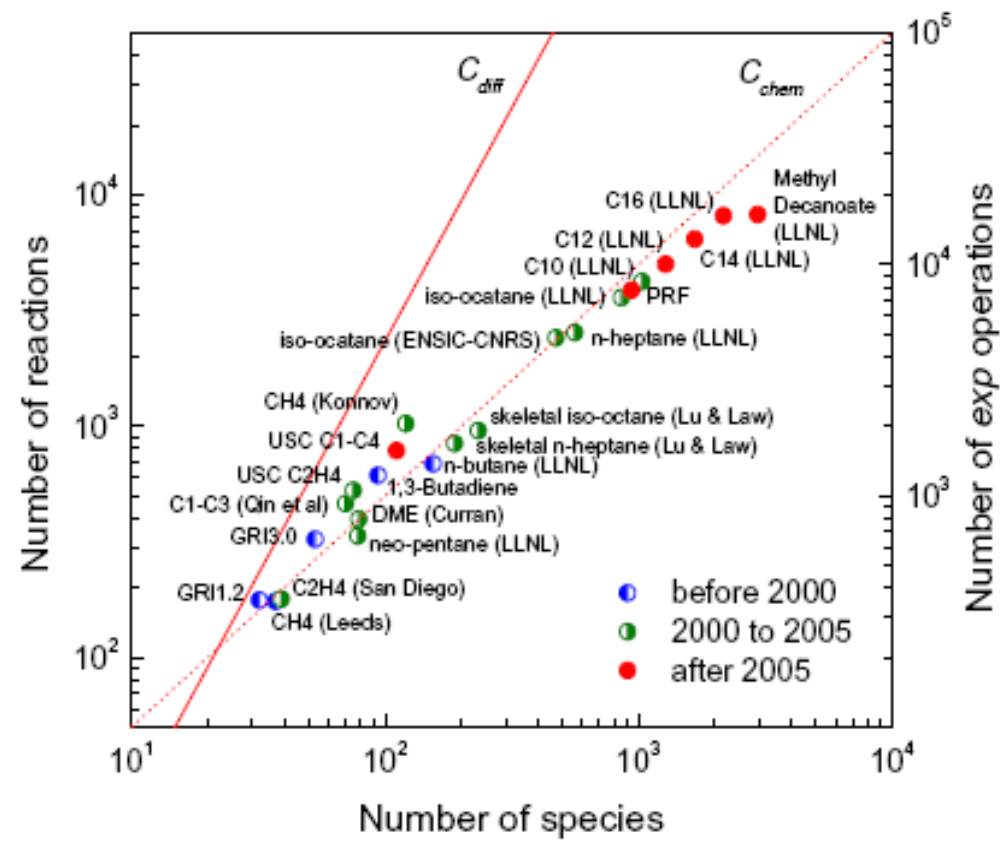

Figure 2.1. Dependence of the Number of Reactions and the Statistical Computational Cost in Commonly used Chemical Mechanisms (Lu, Law and Yoo, et al. 2009).

Figure 2.1 shows the number of exponential operations, in the evaluation of chemical rates and the mixture-averaged diffusion coefficient as a function of the number of chemical species in various detailed and skeletal mechanisms (Lu, Law and Yoo, et al. 2009). The evolution in size of detailed and reduced mechanisms over the past decade can also be seen in Figure 2.1. The size of the mechanisms tends to grow with the size of the fuel molecule; the size also grows with time as new discoveries in chemical kinetics are continuously made. Figure 2.1 also shows a comparison of 
the cost for the evaluation of the chemical source terms and the diffusion coefficient, $C_{\text {chem }}$ and $C_{\text {diff }}$ respectively, for various detailed and skeletal mechanisms. It can be seen that $C_{d i f f}$ is more computationally expensive than $C_{\text {chem }}$ because it scales quadratically with the number of species if detailed transport is involved. In order to reduce the computational cost of diffusion term calculation, a method of diffusive species bundling for mixture averaged model was developed by Lu and Law (Lu and Law, 2007). This model groups the species with similar diffusivities, such that the size of the binary diffusion coefficient matrix is reduced. It was found that n-heptane mechanism can be described by less than 20 species groups thus making the calculation of $C_{\text {diff }}$ much less computationally expensive than $C_{c h e m}$. It was reported by $\mathrm{Lu}$ et al. (Lu, Law and Yoo, et al. 2009) that $C_{\text {chem }}$ scales linearly with the number of species making this term the bottle neck of the combustion simulations.

\subsection{Chemical Reactor Networks}

The chemical reactor network (CRN) concept aids in the modeling of complicated combustion systems by reducing their complexity, i.e. it reduces a three dimensional flow plus chemistry problem to a network of elements which are zero dimensional (perfectly stirred reactor) or one dimensional (plug flow reactor).

The chemical reactor network concept was first proposed by Bragg (Bragg 1953). Bragg applied the CRN or zone modeling to the study of a combustion chamber. The combustion chamber was represented with two reactors; the flame region was represented with a perfectly stirred reactor (PSR), and the post-flame region was modeled with a plug flow reactor (PFR). Experimental verification of the model was carried by Longwell and Weiss (Longwell and Weiss 1955) in their back-mixed well stirred reactor near blow-out conditions where the back mixing of recirculation gas was assumed infinitely fast compared to the controlling chemical reaction rate. 
Numerical modeling of reactors such as PSR and PFR using detailed chemistry has been an active research subject in the past few decades. Significant efforts were put into the development of computer codes capable of modeling chemical kinetics along with other processes such as convection and diffusion of chemical species. As a result of these efforts, Kee et al. developed a computer code called CHEMKIN (Kee, et al. 1996). CHEMKIN can handle chemical mechanisms with a large number of species and reactions and be linked to other computer programs in order to simulate a PSR (Glarborg, et al. 1986) or a PFR (Kee, et al. 1985). The aforementioned computer codes are used to simulate the reactors created from the CRN approach.

Zonal combustion modeling was proposed by Swithenbank et al. (Swithenbank, Poll and Vincent 1973) as an improvement over combustion design using correlation parameters, and experimental testing. The reactor studied by Swithenbank was divided into zones represented by idealized reactor elements, such as PSRs, or PFRs as shown in Figure 2.2.

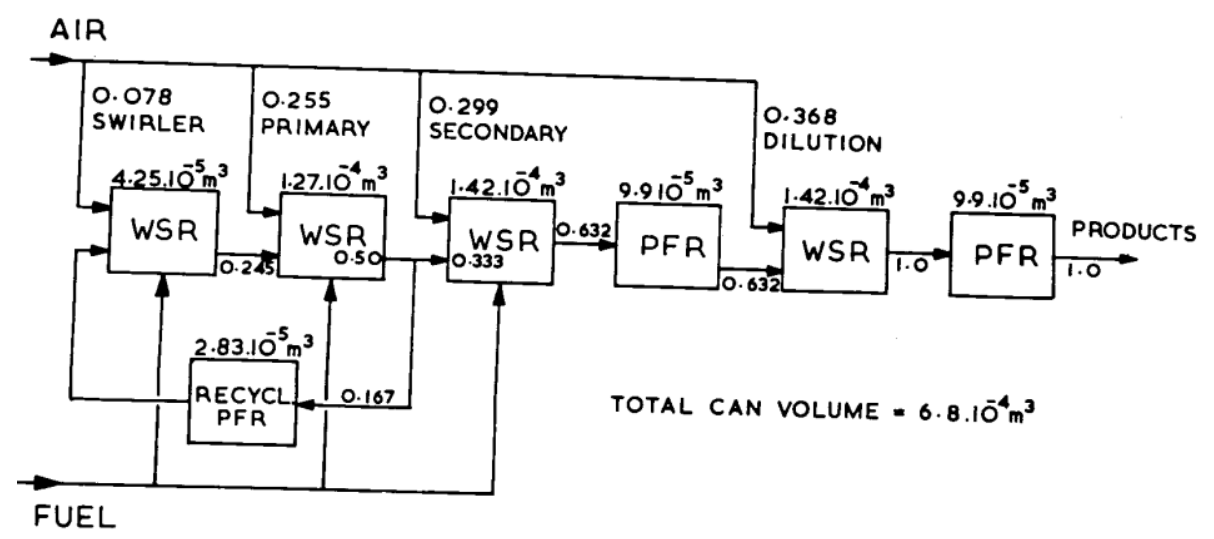

Figure 2.2. Zone Modeling of a Gas-Turbine Can Type Combustor (Swithenbank, Poll and Vincent 1973).

In this network the PSR is represented by the well stirred reactor (WSR). Swithenbank et al. classified the combustor zones depending on the existing mixing level. Zones with high mixing were defined as WSR and zones with low mixing as PFR. This layout allowed Swithenbank to predict the blow-off limits and the combustion efficiency of the system. 


\subsubsection{CRN Application in Gas Turbines}

Rubins and Pratt (Rubins and Pratt 1991) applied zone modeling to simulate an ALF-502 combustor, Figure 2.3. The author tested different possible configurations of the combustor with the goal of reducing carbon monoxide and $\mathrm{NO}_{\mathrm{x}}$ emissions. Their model was composed of PSR, PFR and MIX elements. MIX elements were used to model the later injections of air in the combustor, no chemical reactions occur in these elements only mixing. Rubins and Pratt first performed experiments in the combustor using water and a tracer with the objective to visualize the flow field. Flow visualization helped Rubins and Pratt to propose a zone model layout.

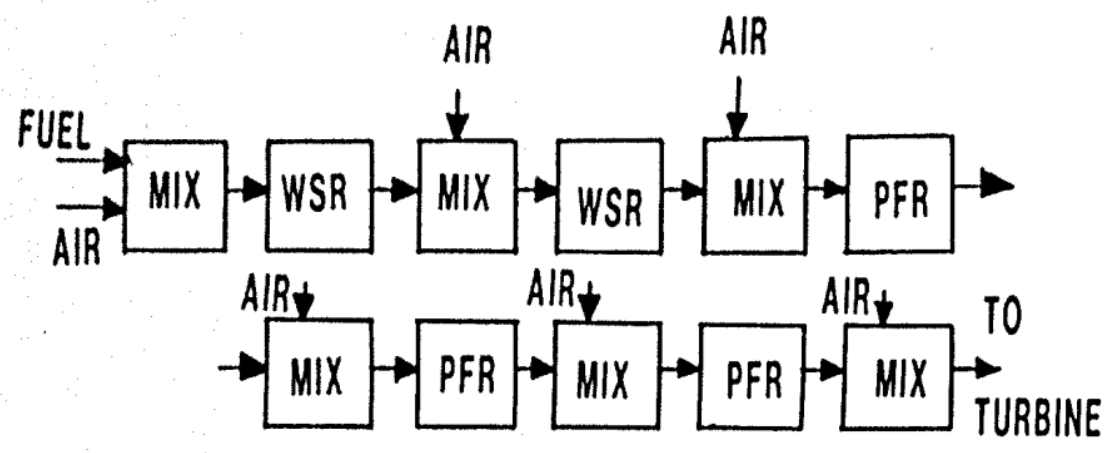

Figure 2.3. Zone Modeling of ALF-502 Gas Combustor (Rubins and Pratt 1991).

Andreini and Facchini (Andreini and Facchini 2004) developed a CRN in order to simulate diffusion flame combustors (MS7001F manufactured by GE) and premixed combustors (LM2500DLE manufactured by GE). Andreini and Facchini developed the CRN using only the combustor geometry and the observations of the flow field (it was not aided by computer simulation data). The CRN model was a collection of PSRs and PFRs and the final CRN computer code was embedded into a main computer code which simulated the entire power generation plant. Results obtained by Andreini and Facchini showed that the model was capable of capturing the $\mathrm{NO}_{\mathrm{x}}$ emissions trend when classical $\mathrm{NO}_{\mathrm{x}}$ reduction methods are used, such as steam or water injection. 
a)

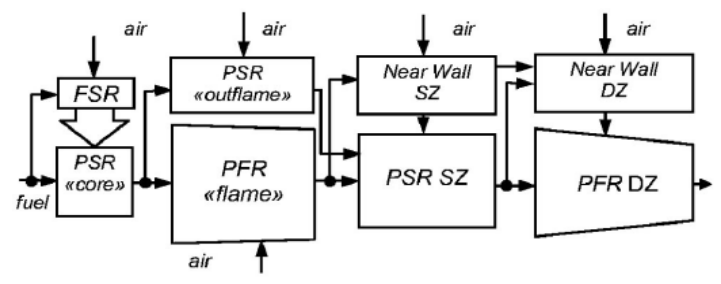

b)

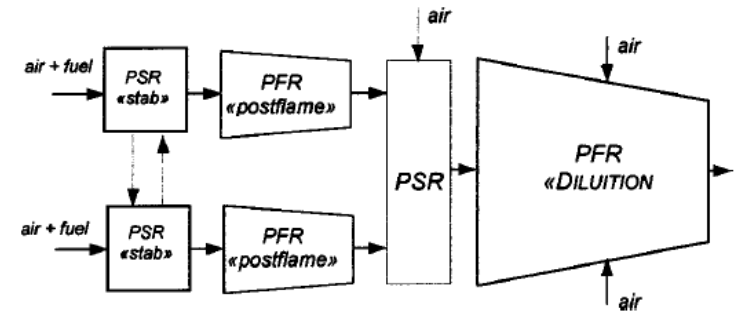

Figure 2.4. CRN Layout of a) Diffusion Combustor, and b) Premixed Combustor (Andreini and Facchini 2004).

Novosselov (Novosselov 2006) applied the CRN concept to model a generic, leanpremixed, single-injector, can-type combustor. The CRN layout was created using data from computer simulations. Novosselov used two variables to perform the clustering of computational cells; temperature and carbon monoxide mass fraction.

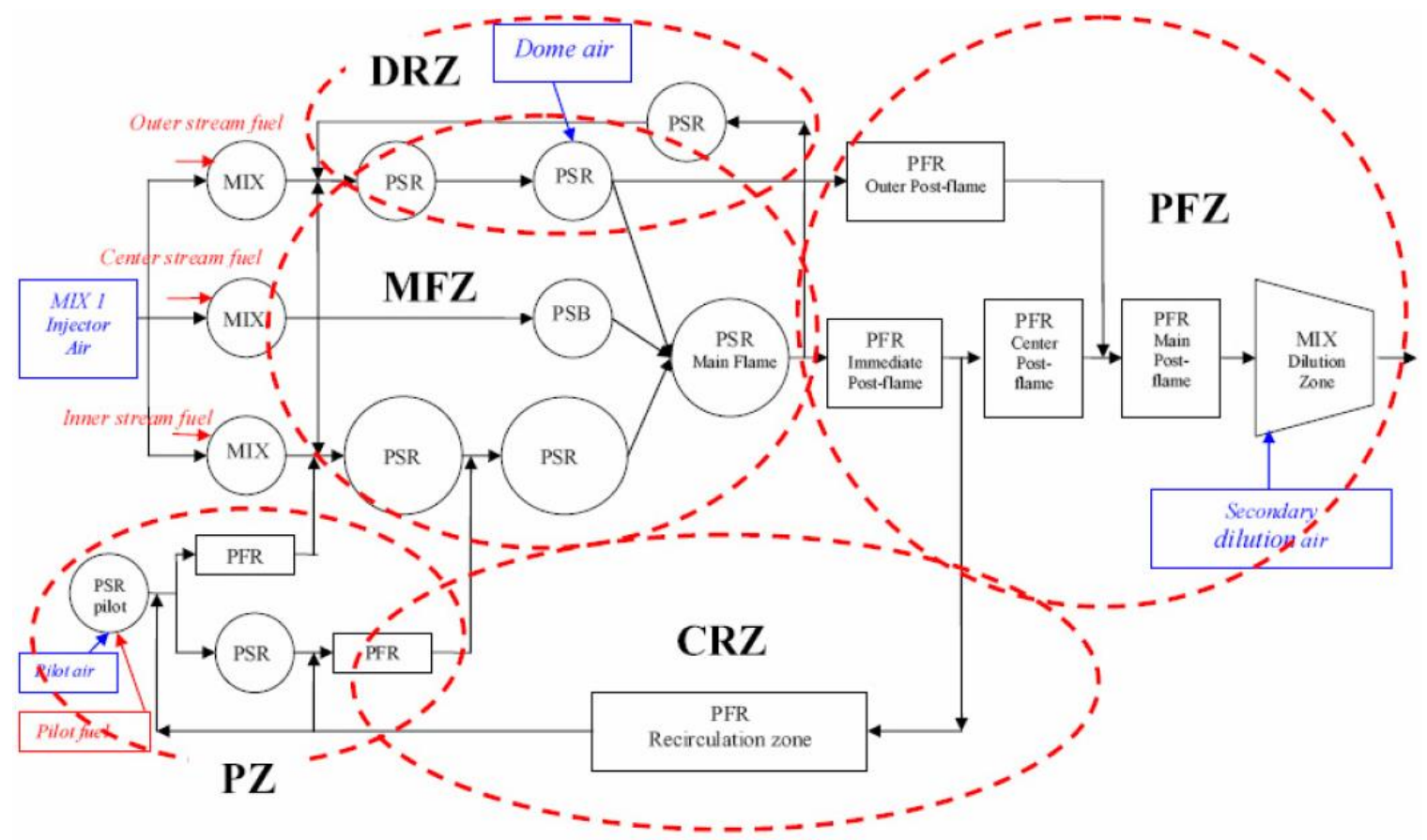

Figure 2.5. Zone Modeling of the Single-Injector, Can-Type, GT Combustor (Novosselov 2006).

The model was tested by comparing the predicted $\mathrm{NO}_{\mathrm{x}}$ against experimental results. Novosselov tuned his model by means of increasing or reducing the volume of zones in order to improve the agreement of the $\mathrm{NO}_{\mathrm{x}}$ predictions with experiments. 
Kanniche (Kanniche 2010) also developed a CRN with the results obtained from CFD simulations. Kanniche performed CFD simulations of a swirl non-premixed combustor using the RANS k-epsilon turbulence model and for the chemistry he used a one step global reaction. The clustering of computational cells was performed using temperature and equivalence ratio variables, and all zones were treated as PSR. The GRI 3.0 (Smith, et al. 1999) detailed mechanism was used to simulate the chemistry in the CRN. The main goal of this study was to investigate the effects of the ambient temperature and relative humidity on $\mathrm{NO}_{\mathrm{x}}$ emissions from a combustor. This work reported the following conclusions; $\mathrm{NO}_{\mathrm{x}}$ emissions increase with the increase of ambient temperature and they decrease with the increase of relative humidity. The predictions of the study were not verified against experimental data.

a)

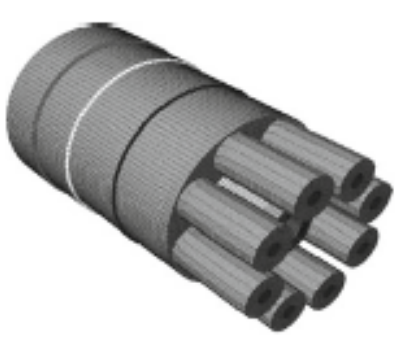

Figure 2.6. a) Geometry of the Combustor, b) CRN Obtained by (Kanniche 2010).

\subsubsection{Other Applications of CRN}

Falcitelli et al. (Falcitelli, Pasini and Tognotti 2002) created a CRN for a glass furnace using information from CFD simulations. Falcitelli divided the furnace into zones using two variables; temperature and equivalence ratio. A third variable, the angle of the velocity vector was employed to determine if the zone corresponded to a PSR or a PFR. The angle of the velocity vector was calculated for each one of the computational cells clustered in one zone of the network. 
If the standard deviation of the velocity angle of the zone was small, then the zone was considered as a PFR, or else the zone was considered as a PSR.

a)

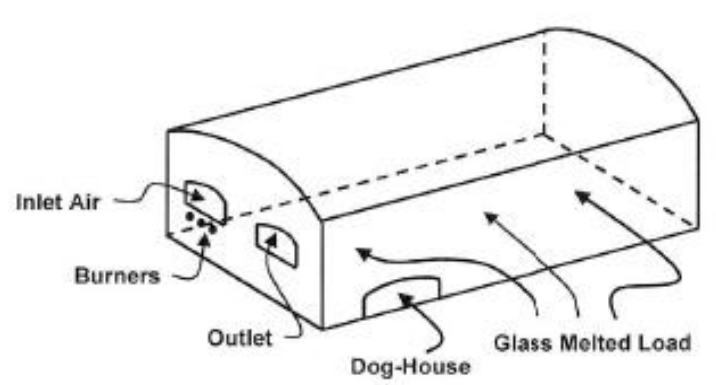

b)

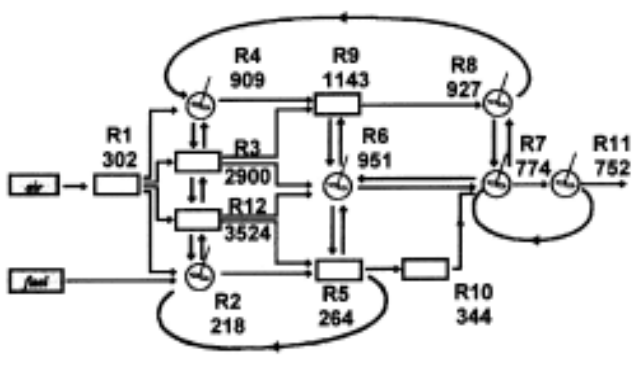

Figure 2.7. a) Furnace Sketch, b) CRN developed by Falcitelli et al. (Falcitelli, Pasini and Tognotti 2002).

Falcitelli et al. built a CRN which consisted of 12 reactors. The CRN was simulated using an in-house chemical mechanism, (Faravelli, et al. 1997), which consists of about 200 species and greater than 3000 reactions and the nitrogen sub-mechanism involves about 200 reactions and 40 species. It is reported that the simulation of the CRN took 75 [min] in a desktop computer (Pentium II). The reported $\mathrm{NO}_{\mathrm{x}}$ simulation values were in good agreement with the experimental values (< $2 \%$ error).

Niksa and Liu (Niksa and Liu 2002) also applied CRN in the computer modeling of furnaces. Niksa and Liu performed the zone division using the information from the characteristic chemical processes present in the combustion chamber, such as; main flame, recirculation zone, mixing layer, etc. Their model was able to depict all the important trends among the major intermediaries and products from a selection of different fuels. However important discrepancies were found in the concentration of gaseous species. The authors explained these differences as a result of the performance of the reaction mechanism used in the simulations. NO predictions were improved by tuning of the reaction mechanism. 


\subsubsection{Commercial $C R N$ codes}

Energico is a commercially available CRN software code developed by Reaction Design (Kee, et al., 2008). Energico creates the CRN using previously obtained CFD simulations results. Using cell information such as oxygen concentration and temperature Energico divides the CFD domain in zones and then using extra variables it describes each zone as PSR or PFR. Finally detailed chemistry simulations are performed in the CRN. This process is basically the same used by Falcitelli et al. (Falcitelli, Pasini and Tognotti 2002) and by Niksa and Lui (Niksa and Liu 2002).

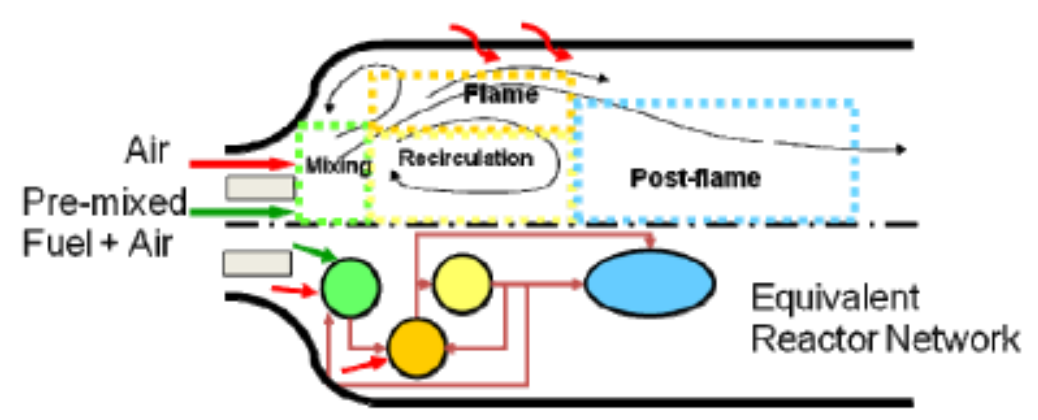

Figure 2.8. CRN Layout Created using Energico Software.

Energico relies on CHEMKIN software to solve the chemistry of the CRN. Energico was specially developed to predict $\mathrm{NO}_{\mathrm{x}}$, carbon monoxide, and unburnt hydrocarbons concentrations.

\subsection{Integration Methods for Stiff Equations}

An extensive description of numerical methods for the integration of stiff equations can be found in Oran and Boris (Oran and Boris 2001). The integration methods can be divided in four categories:

1 Implicit Methods. These methods require that at each time step several internal iterations must be performed in order to find the new value of the dependent variable. It is also necessary to invert the Jacobian matrix. Inversion of the Jacobian matrix usually needs $I^{3}$ steps, $I$ being the number of species, which makes it computationally expensive. The internal iterations for each time step 
require a good initial guess otherwise the solution will diverge from the true solution. The advantage of these methods is that relatively large time steps can be used. An example of this approach is the Newton method.

2 Backward Differentiation Formulas (or Explicit Methods). These methods are the most common methods for solving stiff systems. They do not require internal iterations every time step and most of them are self-starting. However these methods are constrained by the time step size. The most common methods are the backward Euler and Runge-Kutta methods.

3 Exponential Methods. These methods are based on the idea that exact solutions of stiff linear ordinary differential equations (ODEs) behave like a decaying exponential functions. Exponentials are poorly approximated by polynomials when the step size is larger than the smallest characteristic time scale of the system, utilization of exponential approximations should allow considerably larger time steps. These methods can be implicit or explicit.

4 Asymptotic Methods. For cases when the time step is large enough asymptotic solutions are employed. This is the case for problems where the decaying nature of the exponential relaxation modes makes large relative errors progressively less important because the correct equilibrium is being approached. These methods have an advantage over explicit or even implicit methods because they provide greater accuracy at large time steps. However, these methods are not conservative and it is necessary to re-scale the solution in order to maintain stability.

The main challenge for the numerical integration methods is to solve systems that have a wide range of characteristic time scales, stiff systems. All of the methods mentioned before fairly predict the solution of the system as $t \rightarrow \infty$, however transient evolution of the system is not well captured by implicit methods. Explicit methods have the capability to calculate more accurately the transient behavior of the system but they are constrained by small time steps. 
Recently, Gou et al. (Gou, et al. 2010) proposed a method that calculates the transient behavior of stiff systems. The method consists in clustering chemical species in three sets depending on its characteristic time scale. Solution of this system results in the independent integration of the species clusters. For example, small time scale species are integrated in the time interval given by $\tau_{\text {small }}<t<\tau_{\text {medium }}$, medium time scale species $\tau_{\text {medium }}<t<\tau_{\text {large }}$, and finally large time scales species $\tau_{\text {large }}<t<\tau_{\text {flow }}$. This method claims to reduce the computational time by not integrating the entire system of equations every time step.

Other available integration methods of stiff systems like the Runge-Kutta method require the evaluation of the right hand side, RHS, (e.g. sources) up to six times per time step ( $5^{\text {th }}$ order Runge-Kutta method (Cash and Karp 1990)). Multiple evaluation of the RHS increases the CPU time needed to calculate the solution of the system.

A significant effort in the development of algorithms for the solution of stiff systems was conducted at the Lawrence Livermore and Los Alamos National Laboratories. The product of this effort is a library of solution methods for stiff systems called ODEPACK (Hindmarsh 1982). The library contains several solution methods but most of them are variations of the GEAR method (Gear 1971).

\subsection{Reduced Chemical Mechanisms}

Another approach to speed up the solution of this problem is the utilization of one (or two) step global mechanisms, or reduced mechanisms [(Westbrook and Dryer 1981), (Mallampalli, Chen and Fletcher 1998), (Sung, Law and Chen 2001)]. It has to be mentioned that time saved by the utilization of these simplified mechanisms correspond only to the reduction of the total number of chemical species needed to simulate the system. 
One step or two steps global mechanisms (Westbrook and Dryer 1981) contain the chemical species whose concentration is the largest (e.g. methane, oxygen, nitrogen, water, carbon dioxide). These mechanisms are optimized to predict the heat release of the combustion process and also to calculate the laminar flame speed. Computer simulations using reduced mechanism can predict the adiabatic flame temperature, velocity and major species concentration fairly well. But they are limited in the sense that pollutants and trace species are not included (such as NO) in the mechanism. Nevertheless they are used in complex computer simulations where most of the computing power is used in the prediction of the flow field, for example in LES simulations.

Reduced mechanisms are more complete than global reaction mechanisms because they include some chemical species of interest, e.g. $\mathrm{NO}_{\mathrm{x}}$. However, reduced mechanisms failed to predict concentration of pollutants under certain conditions as was demonstrated in a previous work (Escobar, et al. 2011). In this work the $\mathrm{NO}_{\mathrm{x}}$ production for methane-air flame was calculated using two different detailed mechanisms, GRI 3.0 mechanism (Smith, et al. 1999) and San Diego mechanism (UCSD-mech 2012), and two reduced mechanisms ARM19 and ARM9. For a mixture with an equivalence ratio equal to 0.8 (equivalence ratio is defined as the ratio of the fuel-tooxidizer ratio to the stoichiometric fuel-to-oxidizer ratio) and a short residence time in the combustor, it was found that ARM9 was not able to calculate the $\mathrm{NO}_{\mathrm{x}}$ produced by the prompt pathway mainly because this mechanism assumes the concentration of the HCN chemical specie to be in steady state. Despite the limitations of the reduced mechanism, ARM9 is one of the most robust chemical mechanisms for methane-air combustion simulation and is commonly used as an initial step in the numerical simulations solution procedure.

The present study focused on the development of an approach to numerically predict the variables of interest in combustion (e.g. velocity, temperature, chemical species concentration) faster than commonly used methods. In order to achieve this goal different burner set ups were studied using relatively inexpensive computational models (RANS along with one step global 
mechanisms). Then the CRN method was applied in order to predict the chemical species concentration using a detailed chemical mechanism. Finally a novel integration method was implemented in the solution of the main component of the CRN, the perfectly stirred reactor (PSR). Solution of the PSR using this novel integration method was compared with the solution obtained using a commercial software (CHEMKIN). The aforementioned approach is explained in detail in the following chapters along with the presentation of results and discussions.

This study hopes to contribute in the acceleration of combustion simulations by the development of a novel integration method and its application in the main component of the CRN, the perfectly stirred reactor. A library of computer programs with applications to chemical reactions was developed using the LTIM and it is presented in the current work. Finally, an assessment of the computational time needed by a CRN simulation using detailed mechanism (Smith, et al. 1999), CFD simulation using ARM9 (Mallampalli, Chen and Fletcher 1998), and CFD simulation using ARM19 (Sung, Law and Chen 2001) was performed. 


\section{Chapter 3: $\quad$ Burner CFD Simulations}

The first stage of the present study is to perform numerical simulations using a relatively inexpensive computational model (RANS), and one step global mechanisms or reduced chemical mechanisms to model the chemical reactions. In the present study numerical simulations were performed for swirl stabilized combustors. Here the flame stabilization is achieved by imposing a tangential velocity component to the inlet flow and then allowing the flow to go through a sudden expansion. Swirl stabilization is used in combustion applications because the swirling helps to obtain a short flame which improves the homogenization of the mixture and the stabilization of the flame (Frassoldati, et al. 2005). The shape of the flow field in the swirl combustors can be characterized by means of a non-dimensional variable called swirl number. The swirl number is the ratio of axial flow of the tangential component of the momentum to total axial momentum. The dimensionless swirl number is defined as follows.

$$
S=\frac{\int_{0}^{R_{i n}} \rho U W r^{2} d r}{R_{\text {in }} \int_{0}^{R_{i n}} \rho U^{2} r d r}
$$

Where $R_{i n}$ is the inlet radius, $\rho$ is the fluid density, $U$ is the mean axial velocity, $W$ is the mean tangential velocity, and $r$ is the radial distance. The tangential component of the inlet flow is usually applied in combustion applications using two approaches; 1) annular vaned swirler, 2) tangential flow injection. 
a)

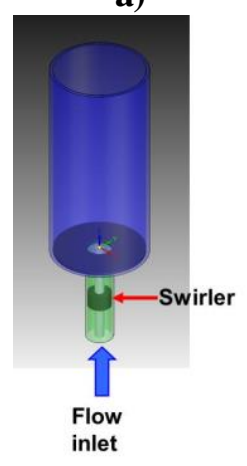

b)

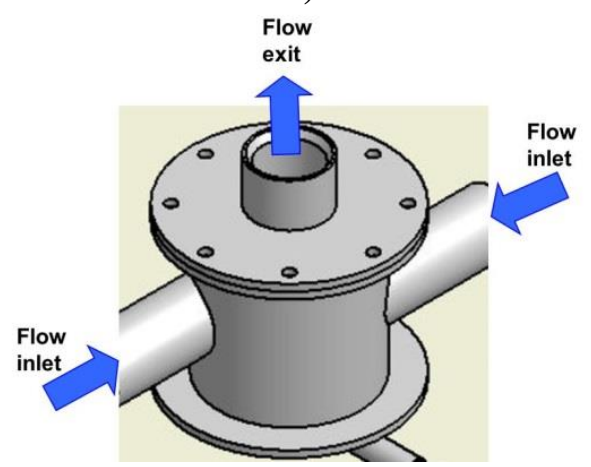

Figure 3.1. Swirl Burner Inlet Configurations. a) Annular Vaned Swirler, b) Tangential Flow Injection (Valera-Medina, et al. 2011).

Two of the burner configurations (NETL, and unconfined burner) used in the present study had a blade swirler to add the tangential velocity component to the flow. Assuming uniform velocity profiles Equation 3.1 can be written in terms of the swirl blade angle, $\alpha_{\text {blade }}$. The resulting swirl number is referred to as the geometric swirl number.

$S_{\text {geom }}=\frac{2}{3} \tan \left(\alpha_{\text {blade }}\right)$

The definition in Equation 3.2 is commonly used when the information about the velocity profiles is not available (Lilley 1977). The flow field is greatly affected by the value of the swirl number, if the swirl number is large enough a central recirculation zone appears. At high swirl numbers $(S>0.6)$ the flow field can be divided into three main zones as seen in Figure 3.2.

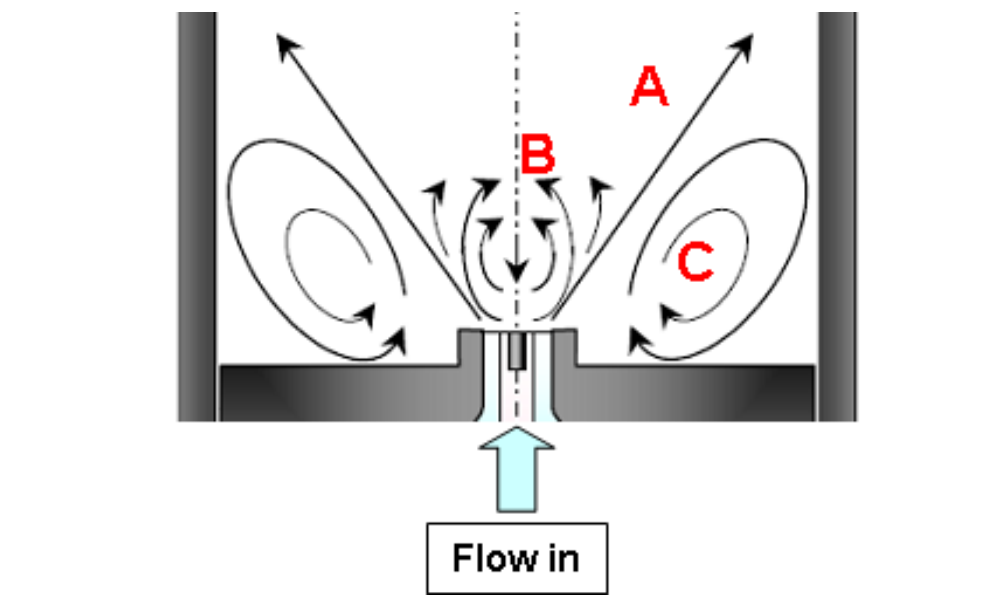

Figure 3.2. Characteristic Flow Field of a Confined Swirl Flame (Meier, et al. 2000). 
The mixing zone (A), shown in Figure 3.2, is where the exothermic reactions mainly take place, the central recirculation zone also known as the main recirculation zone (B) appears when the swirl number is $S>0.6$. The recirculation zone is where the hot intermediate products are transported and the combustion process is completed, and the outer recirculation zones (also known as the secondary recirculation zone) are characterized by containing the burnt gas (C) (Frassoldati, et al. 2005).

Computer simulations of swirl stabilized flames were performed in the present study using a commercial CFD software (ANSYS-FLUENT). Three different burners were studied in order to test the applicability of the chemical reactor network. The burner geometry and simulation details are given in the following sections.

\subsection{Mathematical modeling}

The reacting flow processes that occur in the combustor can be described using the following steady state governing equations: conservation of mass, a scalar transport equation for each one of the chemical species, conservation of momentum, and energy conservation. The steady conservative forms of the governing equations are written as follows:

\section{Continuity Equation}

$\nabla \cdot \rho \mathbf{v}=0$

Where $\rho$ is the density of the mixture, and $\mathbf{v}$ is the velocity vector.

\section{Momentum equation}

$\nabla \cdot \rho \mathbf{v v}=-\nabla P+\nabla \cdot\left(\mu \nabla \mathbf{v}-\rho \overline{\mathbf{v}^{\prime} \mathbf{v}^{\prime}}\right)+\rho \mathbf{g}$

$P$ represents the pressure, $\mu$ is the mixture viscosity, $\mathbf{v}^{\prime}$ is the fluctuating component of the velocity, and $\mathbf{g}$ is the gravity vector. The mixture viscosity was calculated using the ideal gas mixing law 
model and the viscosity of the specie $j$ was calculated using the kinetic theory model. Details of the ideal gas mixing law and the kinetic theory models for the calculation of the viscosity can be found in the user's guide of ANSYS-FLUENT (ANSYS-FLUENT, User's Guide 2013).

\section{Energy equation}

$\nabla \cdot\left(\mathbf{v} \rho c_{p} T\right)=\nabla \cdot\left(\lambda \nabla T-\sum_{j}^{K} h_{j} \boldsymbol{J}_{j}-\rho c_{p} \overline{\mathbf{v}^{\prime} T^{\prime}}\right)+S_{h}$

Where $c_{p}$ is the specific heat of the mixture, $\lambda$ is the thermal conductivity of the mixture, $h_{j}$ is the enthalpy of specie $j, \boldsymbol{J}_{j}$ is the diffusion flux vector of specie $j, K$ is the total number of chemical species, $T^{\prime}$ is the temperature fluctuation, the term $S_{h}$ represents the rate of heat release due the production or consumption of the chemical species. All physical properties are temperature dependent. The calculation of the specific heat of the specie $j\left(c_{p, j}\right)$ was performed using piecewise polynomials and the thermal conductivity $\left(\lambda_{j}\right)$ was calculated using the kinetic theory model. The mixture specific heat $\left(c_{p}\right)$ and the mixture thermal conductivity was calculated using the mixing law and the ideal gas mixing law respectively. Details of the models used to calculate the physical properties in the CFD simulations can be found in the user's guide of ANSYS-FLUENT (ANSYSFLUENT, User's Guide 2013). The variation of, $\rho$, is related to the change of temperature and composition of the flow mixture and is calculated using the ideal gas law.

Equations 3.4, and 3.5 have extra terms related to the turbulence fluctuations (velocity

$\rho \overline{\mathbf{v}^{\prime} \mathbf{v}^{\prime}}$ and temperature $\left.\rho c_{p} \overline{\mathbf{v}^{\prime} T^{\prime}}\right)$. These terms appear after the Reynolds averaging process is applied. The Boussinesq hypothesis is used to evaluate the turbulence related terms as follows.

$-\rho \overline{\mathbf{v}^{\prime} \mathbf{v}^{\prime}}=\mu_{t}\left[2 * S-\frac{2}{3} \nabla \cdot \mathbf{v I}\right]+\frac{2}{3} \rho k$ 
$\boldsymbol{S}=\frac{1}{2}\left(\nabla \mathbf{v}+\nabla \mathbf{v}^{T}\right)$

$\rho c_{p} \overline{\mathbf{v}^{\prime} T^{\prime}}=\lambda_{t} \nabla T$

Where $\mu_{t}$, and $\lambda_{t}$ represent the turbulent molecular viscosity and the turbulent thermal conductivity respectively, $\nabla \mathbf{v}^{T}$ is the transpose of the velocity gradient, $\boldsymbol{I}$ is the unit tensor, and $k$ is the kinetic energy. The calculation of the turbulent viscosity and turbulent thermal conductivity depends on the closure approach used to model turbulence. The closure models used in this study are discussed in the following sections.

\subsection{1 k-epsilon Turbulence Model}

In this study two closure approaches were used; the $k$-epsilon turbulence model and the $k$ epsilon RNG (Re-Normalisation Group) turbulence model. The k-epsilon model (Launder and Spalding 1972) solves the progress of the turbulent kinetic energy in the system $(k)$ and the turbulent dissipation rate (epsilon) by adding two extra equations to the problem.

$\nabla \rho \mathbf{v} k=\nabla \cdot\left[\left(\mu+\frac{\mu_{t}}{\sigma_{k}}\right) \nabla k\right]+G_{k}+G_{b}-\rho \epsilon$

$\nabla \rho \mathbf{v} \epsilon=\nabla \cdot\left[\left(\mu+\frac{\mu_{t}}{\sigma_{\epsilon}}\right) \nabla \epsilon\right]+C_{1, \epsilon} \frac{\epsilon}{k}\left(G_{k}+C_{3, \epsilon} G_{b}\right)-C_{2, \epsilon} \rho \frac{\epsilon^{2}}{k}$

Where $G_{k}$ term is related to the production of turbulence and $G_{b}$ term includes the buoyancy

effects. A detailed description of the turbulence production and buoyancy effects terms can be found in the theory guide of FLUENT (ANSYS-FLUENT, Theory Guide 2009). The values of the 
coefficients used in Equations 3.8 and 3.9 are the standard values recommended by the theory guide of FLUENT software. These values are valid for most of the turbulent flow problems.

The turbulent viscosity and thermal conductivity can be calculated using the following relationships.

$\mu_{t}=\rho C_{\mu} \frac{k^{2}}{\epsilon}$

$k_{t}=\frac{c_{p} \mu_{t}}{P r_{t}}$

Where $C_{\mu}=0.0845$, and the turbulent Prandtl number $P r_{t}=0.85$ are constants.

\subsection{2 k-epsilon RNG Turbulence Model}

The k-epsilon RNG (Re-Normalisation Group) model was also used in a second set of simulations. The k-epsilon $R N G$ is similar to the standard $k$-epsilon model but includes the effect of swirl in the turbulence, and an analytical expression for the calculation of the turbulent Prandtl number.

$\nabla \rho \mathbf{v} k=\nabla \cdot\left[\alpha_{k} \mu_{e f f} \nabla k\right]+G_{k}+G_{b}-\rho \epsilon$

$\nabla \rho \mathbf{v} \epsilon=\nabla \cdot\left[\alpha_{\epsilon} \mu_{e f f} \nabla \epsilon\right]+C_{1, \epsilon} \frac{\epsilon}{k}\left(G_{k}+C_{3, \epsilon} G_{b}\right)-C_{2, \epsilon} \rho \frac{\epsilon^{2}}{k}-R_{\epsilon}$

Where $\alpha_{k}$ and $\alpha_{\epsilon}$ are the inverses of the effective Prandtl number for $k$ and $\epsilon$, respectively. The values of the constants used in the $R N G$ formulation vary from the values used in the standard $k$ epsilon model. One of the important differences is the calculation of the turbulent viscosity, the 
theory behind of the $R N G$ (or renormalization) method results in a differential equation for the turbulent viscosity shown next.

$d\left(\frac{\rho^{2} k}{\sqrt{\epsilon \mu}}\right)=1.72 \frac{\hat{v}}{\sqrt{\hat{v}^{3}-1+C_{v}}} d \hat{v}$

Where $\hat{v}=\frac{\mu_{e f f}}{\mu}$ and $C_{v}=100$. A detailed description of the $k$-epsilon $R N G$ turbulence model can be found in the theory guide of FLUENT software (ANSYS-FLUENT, Theory Guide 2009) and in Orzag et al. (Orszag, et al. 1993).

\subsubsection{Species Transport Modeling}

The evolution of the flow composition in the system is modeled by the species transport equation. FLUENT software solves $N-1$ partial differential equations, where $N$ is the total number of chemical species included in the simulation. The steady state species transport equation is shown next.

$\nabla \cdot\left(\rho \mathbf{v} Y_{i}\right)=-\nabla \cdot \boldsymbol{J}_{i}+R_{i}$

Where $Y_{i}$ is the mass fraction, $\boldsymbol{J}_{i}$ is the mass diffusion flux, and $R_{i}$ is the net rate of production or consumption of the specie $i$.

The mass diffusion flux is modeled using the Fick's law including turbulent mass fluxes

$\boldsymbol{J}_{i}=-\left(\rho D_{i, m}+\frac{\mu_{t}}{S c_{t}}\right) \nabla Y_{i}$

Where $S c_{t}$ is the turbulent Schmidt number which is equal to $S c_{t}=\frac{\mu_{t}}{\rho D_{t}}=0.7$. 
The net rate of production or consumption of the specie $i, R_{i}$, is determined by considering the rate contribution of each reaction in the chemical mechanism used.

$$
R_{i}=M W_{i} \sum_{j=1}^{N_{\text {reac }}} R_{i, j}
$$

The $R_{i, j}$ rate calculation depends on the turbulence-chemistry approach used. The selection of the model also depends on the kind of chemical mechanism used, e.g. one step global reaction mechanism or multi-step reaction mechanism and if the combustion is premixed or non-premixed. Premixed combustion refers to the case where the fuel and oxidizer are mixed before entering the combustion chamber whereas the non-premixed refers to the opposite case. The current study presents three cases where two of them are premixed and one is non-premixed. Due to these conditions two turbulence chemistry models were selected, 1) Finite Rate/Eddy-Dissipation, and 2) Eddy-Dissipation Concept. These models are briefly discussed next:

1.- Finite Rate/Eddy-Dissipation. This model is a combination of two approaches, the finite rate model assumes the reaction is controlled by the chemical kinetics and uses the average temperature and concentration of the species to calculate the rate. The rate of consumption or production of the species is given by the following expression.

$R_{i, j}=\left(v_{i, j}^{P}-v_{i, j}^{R}\right)\left(k_{f, j} \prod_{m=1}^{N_{s p}} c_{m, j}^{\alpha_{m, j}^{R}}-k_{r, j} \prod_{m=1}^{N_{s p}} c_{m, j}^{\alpha_{m, j}^{P}}\right)$

where $v_{i, j}^{P}, v_{i, j}^{R}$ are the stoichiometric coefficients of the specie $i$ in the reaction $j$, the superscripts $P$ and $R$ represent the product or reactant side of the reaction, $c_{m, j}$ is the molar concentration of the specie $m$ in the reaction $j$ and $\alpha_{m, j}$ is the reaction order of the specie $m$ in reaction $j$. The reaction rate constant $k$ is calculated using the Arrhenius equation. 


$$
k_{f, j}=A_{j} T^{\beta_{j}} \exp \left(-\frac{E_{a, j}}{R T}\right)
$$

The variables that appear in the rate expression, $A_{j}, \beta_{j}$, and the activation energy $E_{a, j}$ are obtained from experimental measurements or from theoretical values from ab-initio calculations. As shown in Eq. 3.18 the rate is composed of the forward and reverse reaction rates, and the reverse rate is usually obtained from the following expression.

$$
K_{j}=\frac{k_{f, j}}{k_{r, j}}
$$

Where $K_{j}$ is the equilibrium constant of the reaction $j$.

On the other hand, the Eddy-Dissipation model assumes that mixing of the species controls the rate of the reaction (if it mixes, it reacts). The reaction rate calculation is modified to take into account the degree of mixing of the reactants and the products as it is shown in Equations 3.21, and 3.22 .

$$
\begin{aligned}
& R_{i, j}^{R}=v_{i, j} \rho A_{R, j} \frac{\epsilon}{k} \min \left(\frac{Y_{R}}{v_{R, j} M W_{R}}\right) ; j=1, \ldots, N_{\text {tot_reac }} \\
& R_{i, j}^{P}=v_{i, j} \rho A_{P, j} \frac{\epsilon}{k} \frac{\sum_{P} Y_{P}}{\sum_{m=1}^{N} v_{m, j} M W_{m}}
\end{aligned}
$$

where $A_{R}$ and $A_{P}$ are empirical constants.

The Finite Rate/Eddy-Dissipation model uses the smallest rate calculated either by the finite rate or the eddy dissipation model, $\min \left(R_{i, j}, R_{i, j}^{R}, R_{i, j}^{P}\right)$. However, this method is not suitable 
for multi-step chemical mechanisms. It ignores the difference in the reaction rate of different reactions, when the controlling step is the mixing rate of the species. For this reason it should be used for one or two step reaction mechanisms only. A detailed description of the Eddy-Dissipation model can be found in Magnussen and Hjertager (Magnussen and Hjertager 1977).

2.- Eddy-Dissipation Concept (EDC). This method was developed by Magnussen (Magnussen, 1981) and it assumes that the reactions take place in the fine scales which are related to the characteristic turbulent length and time scale. The turbulent length and time scales are calculated using the following expressions

$\xi^{*}=C_{\xi}\left(\frac{v \epsilon}{k^{2}}\right)^{\frac{1}{4}}$

$$
\tau^{*}=C_{\tau}\left(\frac{\nu}{\epsilon}\right)^{\frac{1}{2}}
$$

Where $C_{\xi}, C_{\tau}$ are constants and $v$ is the kinematic viscosity. Combustion in the turbulent fine scales is assumed to occur as that in a constant pressure reactor with initial conditions taken as the current specie concentrations and temperature values stored in the computational cell. The consumption or production rates of chemical species are calculated using the finite rate model along with the Arrhenius rates; the flow residence time is given by the characteristic turbulent time scale. The reaction proceeds in each computational cell until the calculated residence time is reached, the integration is performed using a tabulation integration method better known as ISAT (Pope 1997). Finally the rate of the specie $i$ is modified in order to include the effect of the turbulence in the chemical reaction. 
$R_{i}=\frac{\rho\left(\xi^{*}\right)^{2}}{\tau^{*}\left\lfloor 1-\left(\xi^{*}\right)^{3}\right\rfloor}\left(Y_{i}^{*}-Y_{i}\right)$

$Y_{i}^{*}$ is the fine-scale mass fraction of the specie $i$ after reacting over the time $\tau^{*}$. EDC implementation details are explained in more detail in the theory guide of FLUENT (ANSYSFLUENT, Theory Guide 2009).

\subsection{Confined Premixed Swirl Burner}

\subsubsection{Geometry Details and Operational Conditions}

This burner will be referred in the present study as the NETL (National Energy Technology Laboratory) burner. This is a laboratory scale methane-air burner located at NETL-Morgantown. NETL burner is an atmospheric swirl combustor (closed-open configuration) which consists of a quartz tube that confines the flame and also provides optical access. The quartz tube has an inner diameter of $80.5[\mathrm{~mm}]$ and is $230[\mathrm{~mm}]$ in length. The inlet nozzle and center body, which provides anchor for the swirler and flame, has a diameter of $21.8[\mathrm{~mm}]$ and $8.8[\mathrm{~mm}]$, respectively. The end of the center body and the dump plane are co-planar. A vane type swirler with a geometric swirl number $\left(S_{\text {geom }}\right)$ equal to 0.88 is placed $50[\mathrm{~mm}]$ upstream of the dump plane. Figure 3.3 shows the combustor setup and the swirler geometry.

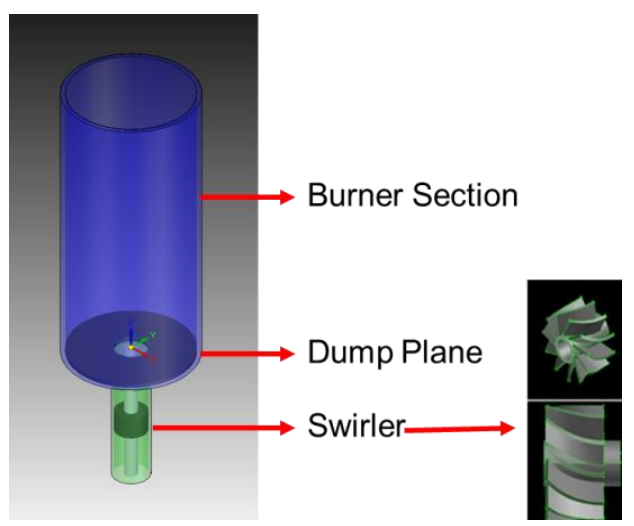

Figure 3.3. NETL Burner Sketch. 
An adiabatic dump plane was used for the present simulations and the total flow rate was set to a constant of 100 [slpm] which gave a Reynolds number based on the hydraulic diameter of 4,385. Simulations were performed using methane as fuel.

\subsubsection{Simulation Details}

Numerical simulations were performed in two steps:

- First, the cold flow simulations were calculated using a three dimensional geometry which included the inlet nozzle, the swirler, and part of the combustor chamber. Simulation results were used to characterize the three dimensional flow inside the combustor. Velocity profiles were extracted at a line located in the middle of the distance between the swirler and the dump plane, Figure 3.4 a).

- Second, the reacting flow was simulated using a two dimensional axisymmetric geometry. The geometry included a small portion of the inlet nozzle and the combustion chamber. Profiles of the three velocity components extracted from the non-reacting three dimensional simulations were used as inlet boundary conditions for the two dimensional axisymmetric simulations. 
a)

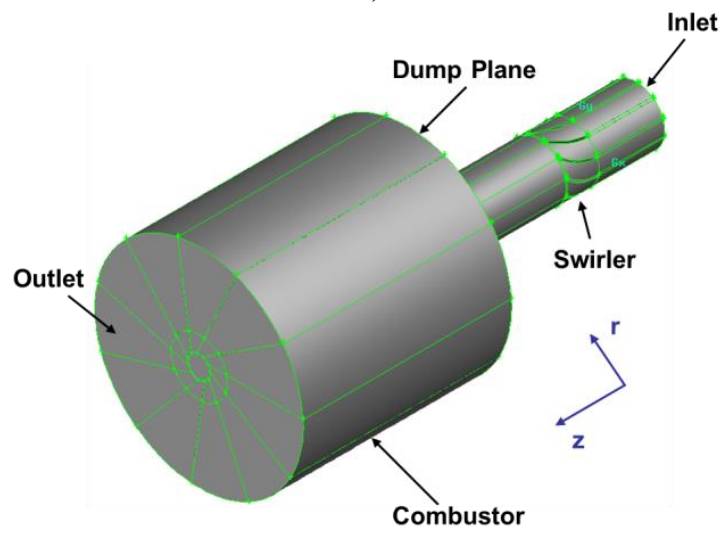

b)

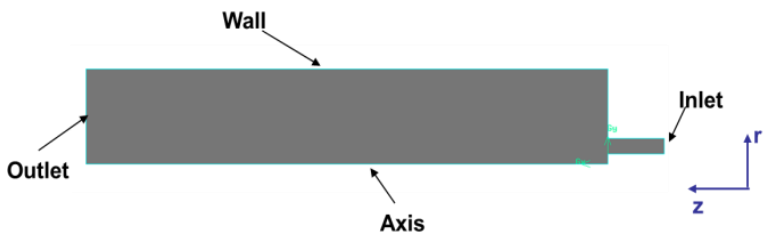

Figure 3.4. CFD Simulations of a) Three Dimensional Inlet Section used for Non-Reacting Flow Simulations and b) Two Dimensional Axisymmetric Combustor Section used for the Reacting Flow Simulation.

Figure 3.4 shows the CFD geometries used, a) three dimensional non-reacting flow simulation and b) two dimensional axisymmetric simulations. The three dimensional inlet section has 800,000 hexahedral cells while the two dimensional axisymmetric section has approximately 48,000 triangular cells.

Table 3.1. Confined Premixed Swirl Burner Simulation Details.

\begin{tabular}{|c|c|c|}
\hline & Non-Reacting Flow Simulation & Reacting Flow Simulation \\
\hline Computational Domain & Three Dimensional & Two Dimensional Axisymmetric \\
\hline Time Formulation & Steady State & Steady State \\
\hline Pressure-Velocity Coupling & SIMPLE & PIMPLE \\
\hline Pressure Discretization Scheme & PRESTO & $2^{\text {nd }}$ Order Upwind \\
\hline $\begin{array}{c}\text { Convective Term Discretization } \\
\text { Scheme }\end{array}$ & $2^{\text {nd }}$ Order Upwind & $k$-epsilon $R N G$ \\
\hline Turbulence Model & $k$-epsilon $R N G$ & $\begin{array}{c}\text { a) One Step Global Mechanism } \\
\text { b) ARM9 } \\
\text { c) ARM19 }\end{array}$ \\
\hline $\begin{array}{c}\text { Chemical Mechanism } \\
\text { Nurbulence Chemistry Interaction } \\
\text { Model }\end{array}$ & N/A & Eddy-Dissipation Concept (EDC) \\
\hline Chemical Species Integration & N/A & ISAT \\
\hline
\end{tabular}

The three dimensional cold flow simulations were performed using the k-epsilon $R N G$ model, while the two dimensional combustion simulations were performed using the k-epsilon RNG turbulence model along with the Eddy-Dissipation Concept (EDC) for the turbulence-chemistry interaction. 
Chemical reactions were modeled using three chemical mechanisms, 1) one step global mechanism (Westbrook and Dryer 1981), 2) a 9 species - 5 reactions reduced mechanism (ARM9) (Mallampalli, Chen and Fletcher 1998), and 3) a 19 species - 15 reactions reduced mechanism ARM19 (Sung, Law and Chen 2001). Second order upwind discretization was used for all equations along with the SIMPLE algorithm for the velocity-pressure coupling.

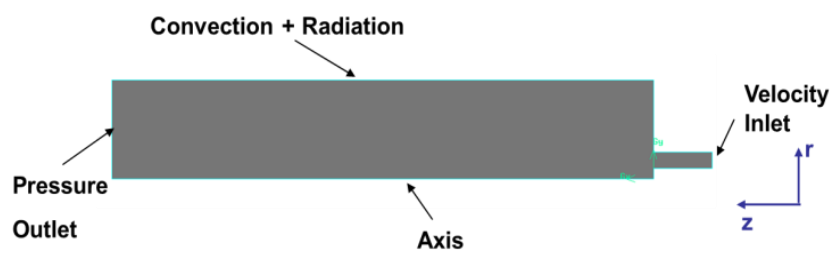

Figure 3.5. NETL Burner Boundary Conditions.

Boundary conditions used in the two dimensional case are shown in Figure 3.5. On the combustor wall a mixed thermal boundary condition is used to include heat loss due to convection and radiation as the quartz tube combustor is not cooled. A heat transfer coefficient of $40\left[\mathrm{~W} / \mathrm{m}^{2} \mathrm{~K}\right]$ was used and heat transfer due to radiation was calculated using the following expression.

$q_{\text {rad }}=\varepsilon_{\text {ext }} \sigma\left(T_{\infty}^{4}-T_{w}^{4}\right)$

where $\varepsilon_{\text {ext }}$ is the emissivity of the external wall, in this case it was set as $\varepsilon_{e x t}=1.0, \sigma$ is the StefanBoltzman constant, and $T_{\infty}$ and $T_{w}$ are the ambient and wall surface temperatures, respectively. The thermal conductivity of the combustor wall is set to $1.92[\mathrm{~W} / \mathrm{m} * \mathrm{~K}]$ to match that of quartz (Thermal Properties of Fused Quartz 2014) and the rest of the walls are considered to be adiabatic.

The inlet boundary composition was set to an equivalence ratio equal to 0.8 of methane-air mixture. The equivalence ratio is defined as follows 
Eq.rat. $=\frac{\left(\frac{m_{\text {oxidizer }}}{m_{\text {fuel }}}\right)_{\text {stoic }}}{\left(\frac{m_{\text {oxidizer }}}{m_{\text {fuel }}}\right)_{\text {actual }}}$

The subscript stoic refers to stoichiometric conditions. The stoichiometric mixture of a hydrocarbon fuel reacting with air can be calculated using the following reaction

$C_{x} H_{y}+a\left(O_{2}+3.76 N_{2}\right) \rightarrow x C O_{2}+\left(\frac{y}{2}\right) H_{2} O+3.76 a N_{2}$

The variable $a$ in Equation 3.28 is calculated from $a=x+y / 4$. In the present study methane is used as fuel $\left(\mathrm{CH}_{4}\right)$ which yields $a=2$. The mass of oxidizer shown in Equation 3.27 is calculated for the stoichiometric mixture in the following fashion, $m_{\text {oxidizer }}=a *\left(M W_{O 2}+3.76 * M W_{N 2}\right)$ where $M W_{i}$ is the molecular weight of the specie $i$.

Finally, the inlet volumetric flow rate of 100 [slpm] was set giving a Reynolds number based on the hydraulic diameter equal to $\left(\operatorname{Re}_{D h}\right)=4,385$.

\subsubsection{CFD Results for Non-Reacting Flow - NETL Burner}

A grid convergence study was performed for the three dimensional cold flow simulations using three different mesh resolutions. Approximately 400,000 cells, 800,000 cells, and 1.6 million computational cells were used (Mesh 1, 2, and 3 respectively). Figure 3.6 shows the calculated axial velocity profiles at the dump plane for the non-reacting flow simulation. 


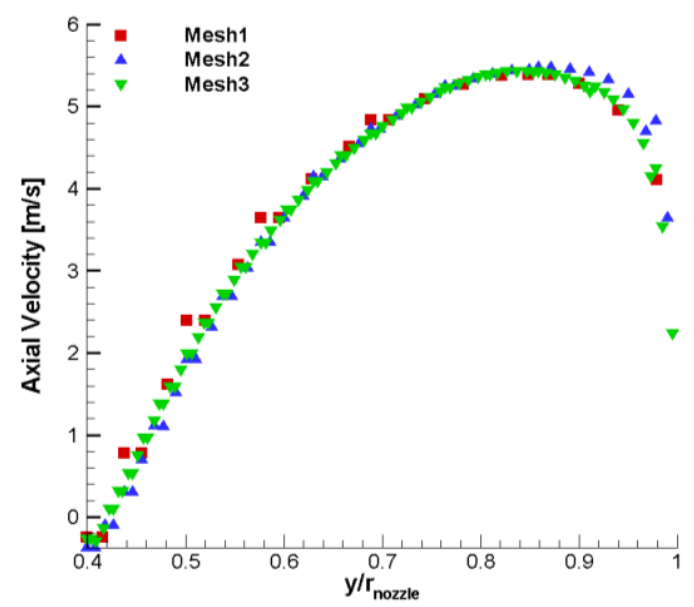

Figure 3.6. Grid Independence of Axial Velocity at Dump Plane, theta $=0$ [deg].

Similar axial velocity profiles were obtained from the three meshes used as it can be seen in Figure 3.6 showing that nearly a grid independent solution was achieved. The shape of the axial velocity profile shows the maximum value near the outer wall $\left(y / r_{\text {nozzle }}=1\right)$ in contrast to the classical velocity profile where the maximum is at the center of the annulus for the case of zero swirl. The distortion of the axial velocity profile from the typical turbulent velocity profile is a function of the swirl number (Chigier and Beer 1964).

Experimental Particle Image Velocimetry (PIV) data (see measured axial velocity contour in Figure 3.7) was used to assess the numerical predictions. Strakey (Strakey 2010) facilitated the experimental results which were obtained for the same non-reacting flow conditions. 


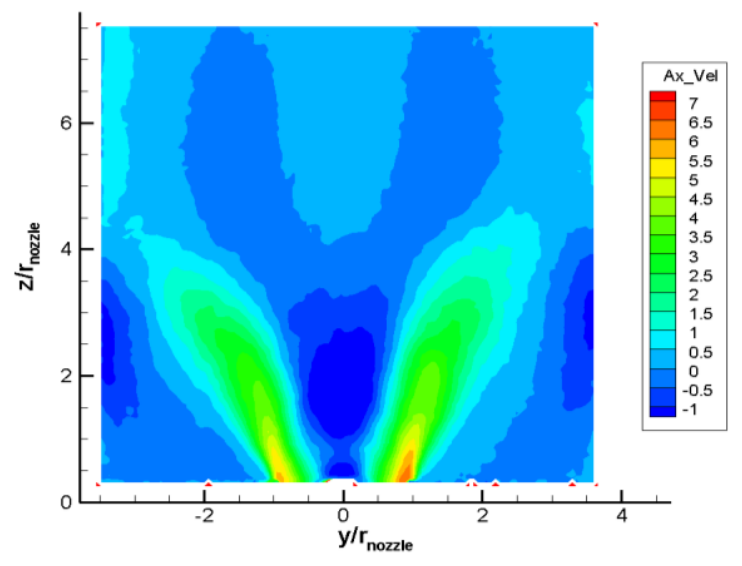

Figure 3.7. PIV Contour of Cold Flow Axial Velocity [m/s], theta $=0$ [deg] (Strakey 2010).

Figures 3.8 a) and $3.8 \mathrm{~b}$ ) show the comparison of the axial and radial velocity profiles obtained from the numerical simulations (Mesh 2) with the experimental values at 1.5 [mm] downstream of the dump plane. Results suggest a good qualitative and a reasonable quantitative agreement with the experiments. Figure 3.8 c) shows a comparison of the tangential velocity profiles at $1.0[\mathrm{~mm}]$ downstream the dump plane. For peak tangential velocity, there is an approximate $25 \%$ difference between the experiments and the computations. It is to be noted that the line profiles are captured at a particular axial-radial plane of the three dimensional simulations upstream of the dump plane (theta $=0[\mathrm{deg}])$.

a)

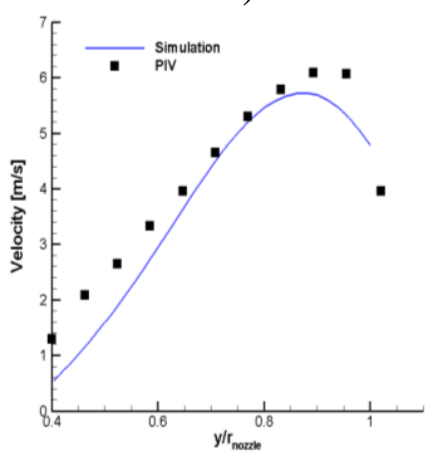

b)

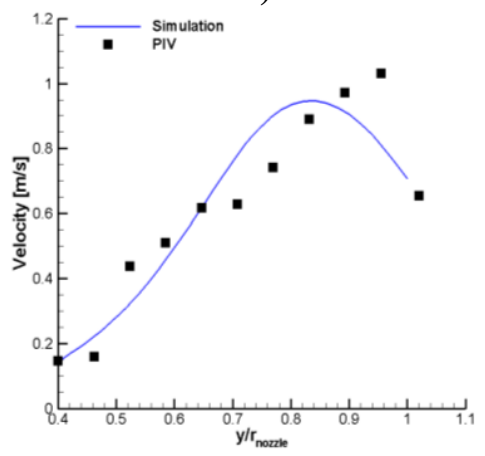

c)

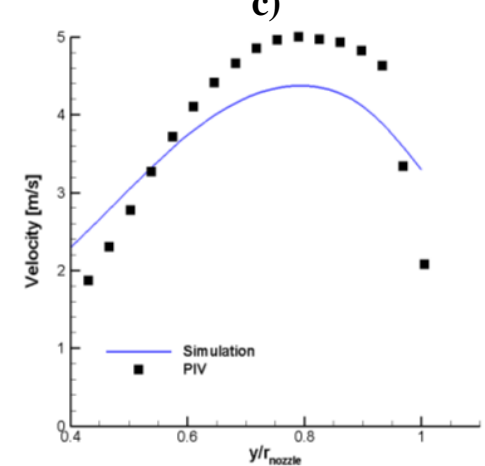

Figure 3.8. Comparison of the PIV Measurements and the Predicted Non-Reacting Flow Velocity Profiles a) Axial Velocity, b) Radial Velocity, and c) Tangential Velocity. 
Figure 3.9 shows predicted velocity profiles plotted at different theta locations along the tangential direction. These velocity profiles were taken in the middle of the distance between the swirler and the dump plane, information from these profiles was used as boundary condition in the two dimensional axisymmetric simulations. From Figure 3.9 it can be seen that there is some scatter in the values of the axial, radial and tangential velocities. Velocity profiles obtained from the three dimensional non-reacting flow simulations were averaged along theta direction (see Figure 3.9) and the average flow profiles were used as inlet boundary conditions for the two dimensional axisymmetric reacting flow simulations (Escobar, et al. 2010). These profiles were extracted from a radial-tangential plane located at $25[\mathrm{~mm}]$ upstream the dump plane and averaged along the eight lines equally spaced in the theta direction.
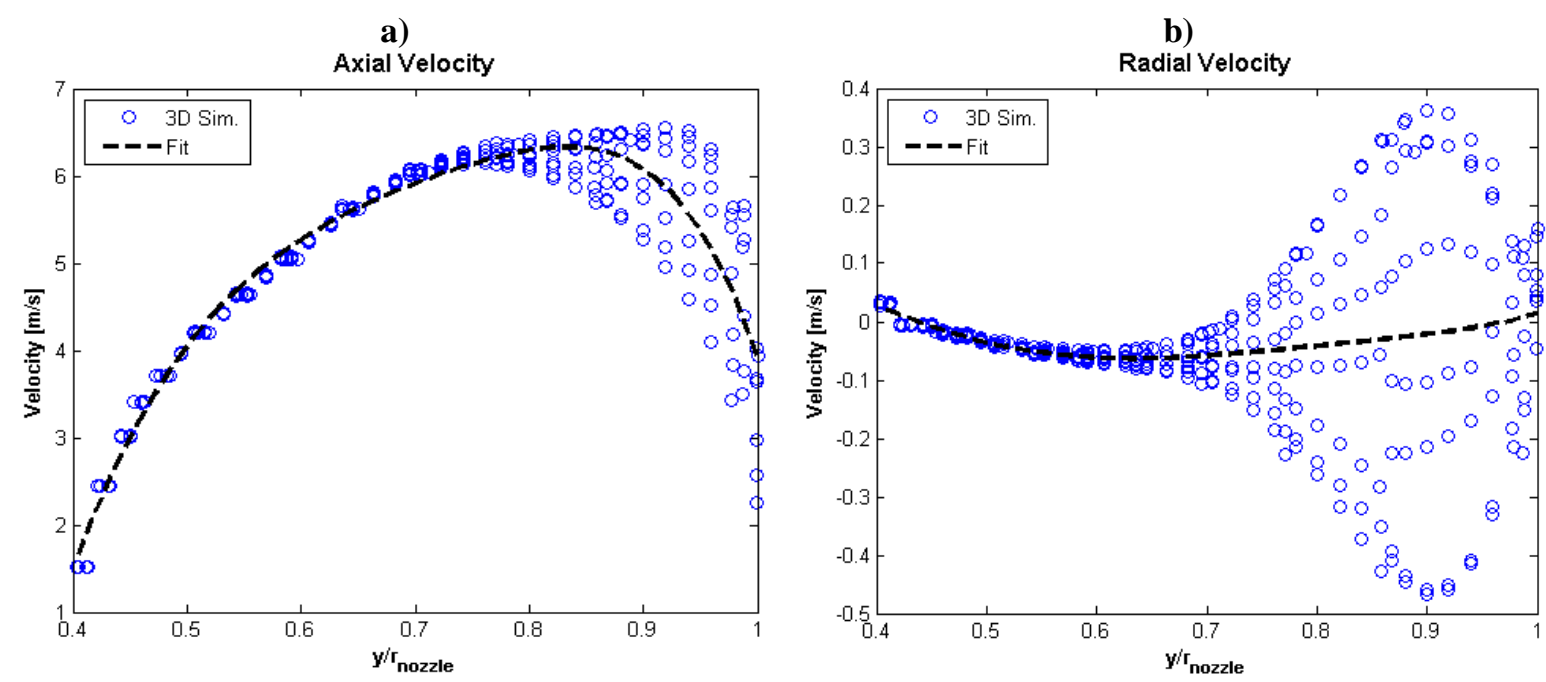


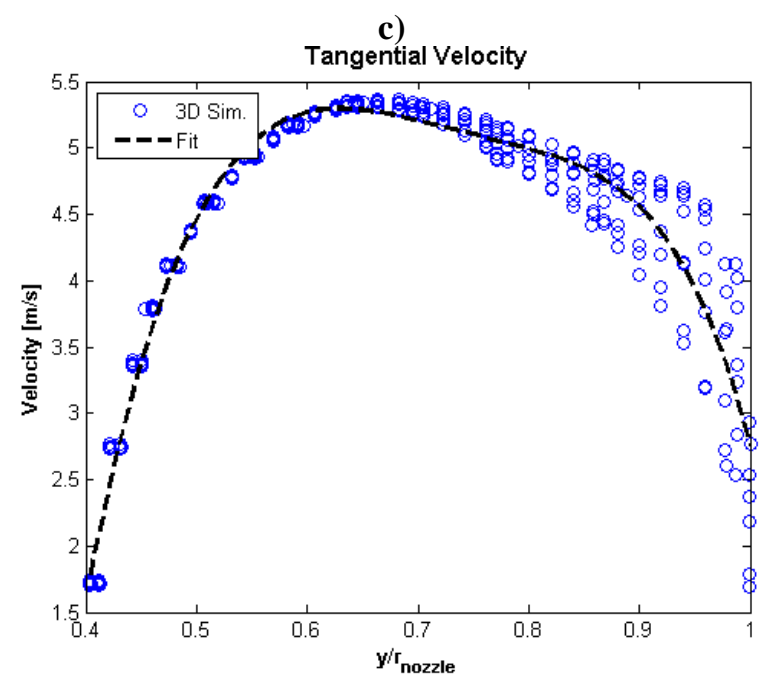

Figure 3.9. Averaged Velocity Profiles $[\mathrm{m} / \mathrm{s}]$ along Theta Direction, a) Axial Velocity, b) Radial Velocity, and c) Tangential Velocity.

Figure 3.10 a) shows the lobed nature of the velocity flow field due to the swirler located at $25[\mathrm{~mm}]$ upstream of the dump plane, midway between the swirler and the dump plane, and at the dump plane Figure 3.10 b).

a)

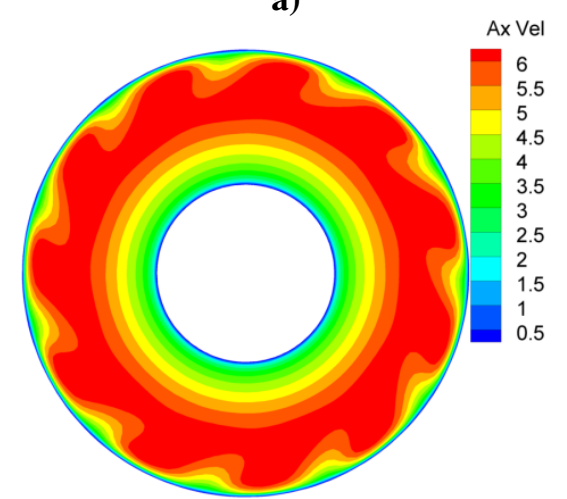

b)

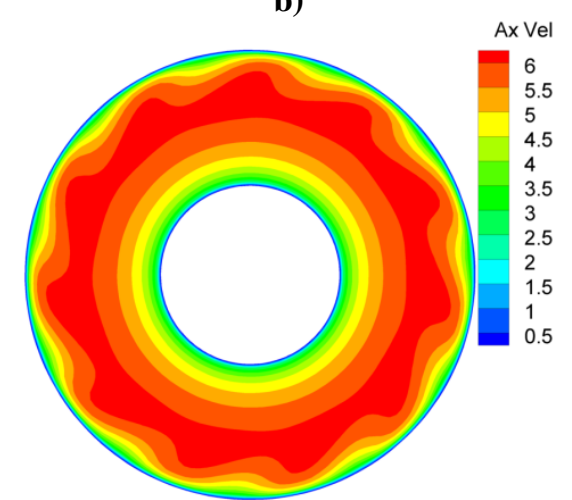

Figure 3.10. Axial Velocity Contours $[\mathrm{m} / \mathrm{s}]$ from Three Dimensional Non-Reacting Flow Simulation at; a) 25 [mm] Upstream of the Dump Plane, b) Dump Plane.

\subsubsection{CFD Results for Reacting Flow - NETL Burner}

Reacting flow simulations were performed with three chemical mechanisms for a methaneair flame with an equivalence ratio equal to 0.8 . Figure 3.11 shows the axial velocity contour with stream lines for the reacting flow case in which the chemistry was modeled using a one step global 
mechanism. Results show two recirculation zones; one close to the axis of the domain (main recirculation zone) and another on the top left corner of the burner (secondary recirculation zone). The main recirculation zone is produced by the vortex breakdown, which is a typical feature of the flow field for swirl flows with high swirl numbers $(S>0.6)$ (Syred and Beer 1974). The general structure of the velocity field remained nearly the same for the three cases with different chemical mechanisms.

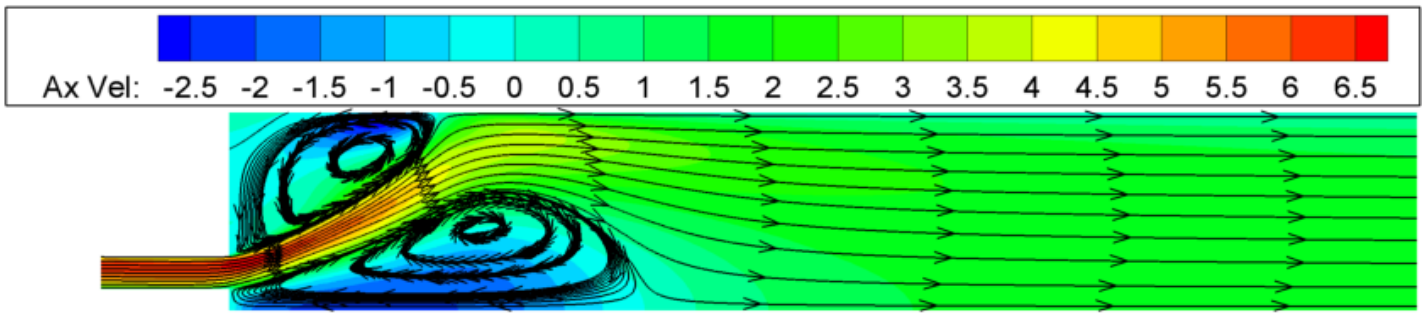

Figure 3.11. Axial Velocity Contour $[\mathrm{m} / \mathrm{s}$ ] with Streamlines from Two Dimensional Axisymmetric Reacting Flow Simulations. One Step Global Mechanism.

The temperature contour obtained using the one step global mechanism is shown in Figure 3.12 a). The maximum temperature region is located at the main recirculation zone which is expected because of the high mixing of the combustion products. The temperature on the secondary recirculation zone is not as high as in the main recirculation zone because of the heat flux lost from the burner to the surroundings. 
a)

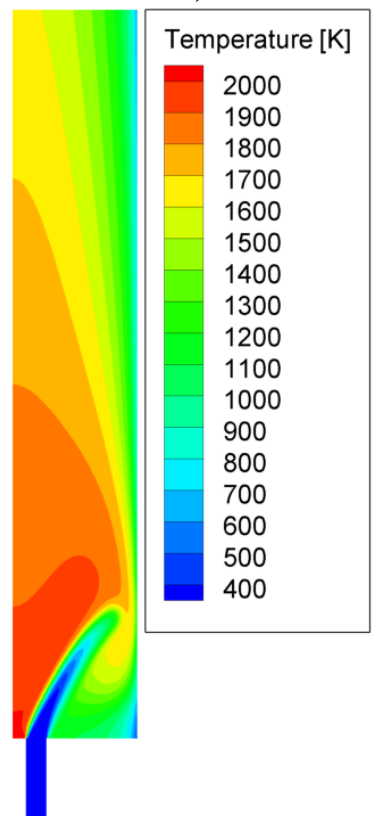

b)

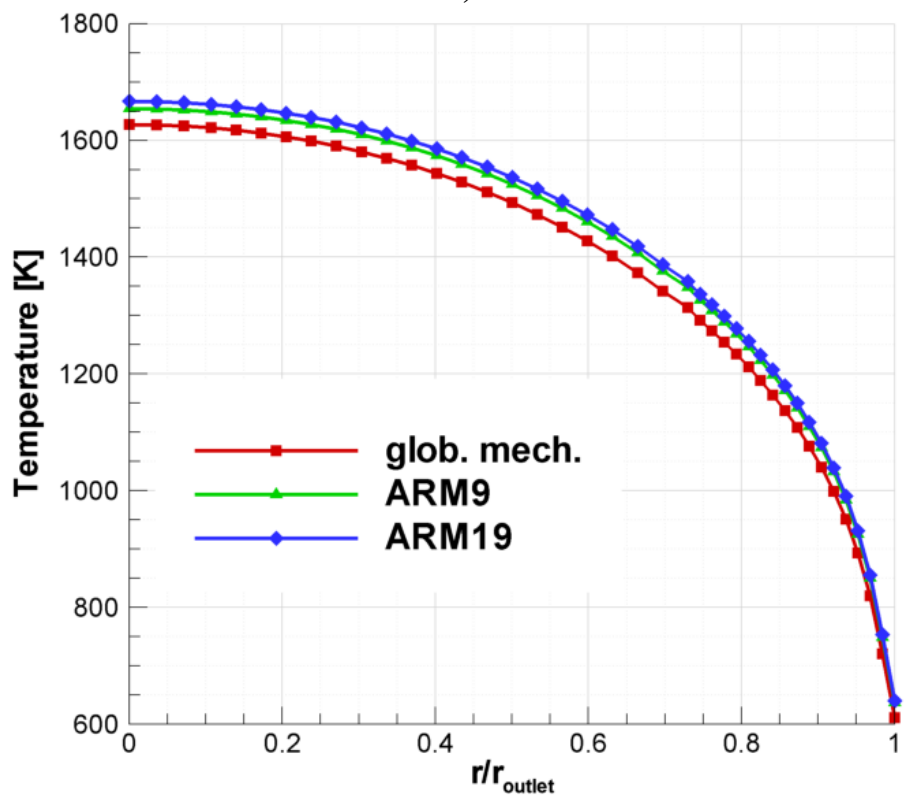

Figure 3.12. Two Dimensional Axisymmetric RANS Simulations. a) Temperature Contours One Step Global Mechanism, b) Outlet Temperature Profile using Different Chemical Mechanisms.

The temperature profiles at the outlet of the NETL burner are shown in Figure $3.12 \mathrm{~b}$ ). In the profile picture, the location $r / r_{\text {outlet }}=0$ is the symmetry axis and $r / r_{\text {outlet }}=1$ is the quartz wall. ARM19 predictions calculated the highest temperature at the center of the domain (symmetry line), the difference with the global mechanism is about $2.44 \%$ according to Equation 3.29.

$\operatorname{Diff} f_{T, \%}=a b s\left[\frac{\left.T_{A R M 19}\right|_{r / r_{\text {outlet }}=0}-\left.T_{\text {glob.mech. }}\right|_{r / r_{\text {outlet }}=0}}{\left.T_{\text {glob.mech. }}\right|_{r / r_{\text {outlet }}=0}}\right] * 100$

Figure 3.13 a) and b) shows the predicted water and oxygen mole fraction profiles located at the outlet of the burner. 
a)

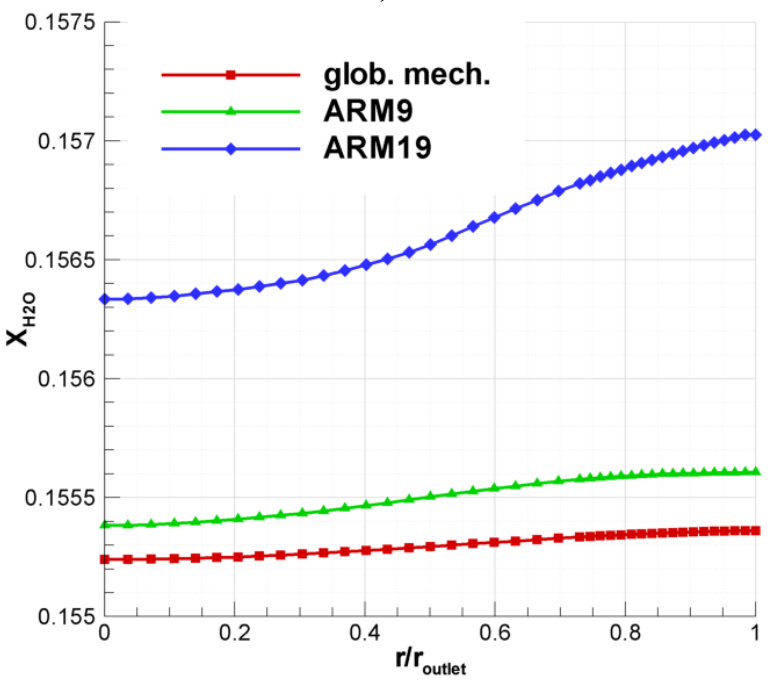

b)

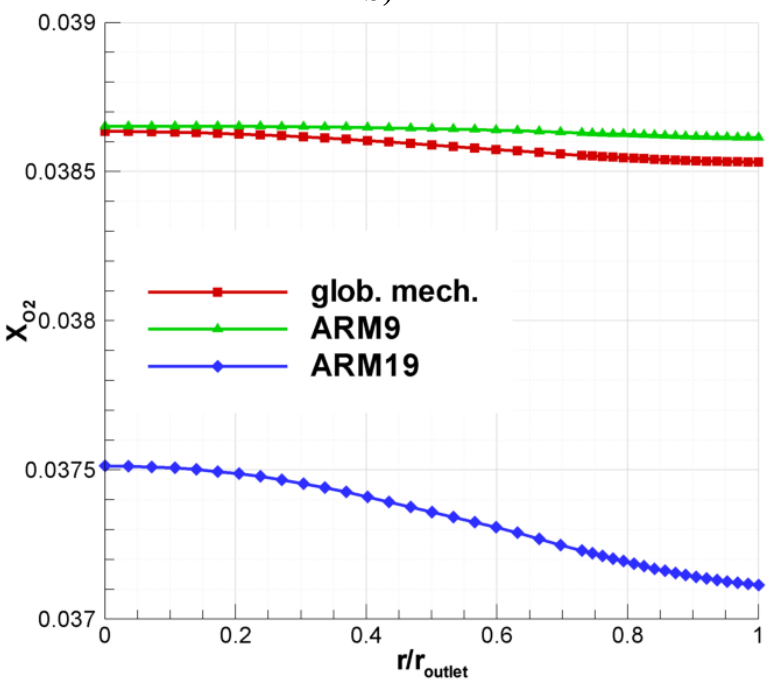

Figure 3.13. Outlet Mole Fraction Profile using Different Chemical Mechanisms. a) Water, b) Oxygen.

Variation of the water and oxygen mole fraction at the outlet of the burner is not significant. Predicted profiles for using these chemical models do not show a significant variation neither for temperature nor mass fraction for the major species. The maximum difference in the water mole fraction is $1.07 \%$, Equation 3.30, and for oxygen is $4.03 \%$, Equation 3.31 .

$$
\begin{aligned}
& \operatorname{Diff}_{X_{H_{2} O}, \%}=\operatorname{abs}\left[\frac{\left.X_{H_{2} O, A R M 19}\right|_{r / r_{\text {outlet }}=1}-\left.X_{\mathrm{H}_{2} \text { O, glob.mech. }}\right|_{r / r_{\text {outlet }}=1}}{\left.X_{\mathrm{H}_{2} \text { O, glob.mech. }}\right|_{r / r_{\text {outlet }}=1}}\right] * 100 \\
& \operatorname{Diff}_{X_{O 2}, \%}=\operatorname{abs}\left[\frac{\left.X_{O_{2}, A R M 19}\right|_{r / r_{\text {outlet }}=1}-\left.X_{O_{2}, A R M 9}\right|_{r / r_{\text {outlet }}=1}}{\left.X_{O_{2}, A R M 9}\right|_{r / r_{\text {outlet }}=1}}\right] * 100
\end{aligned}
$$

Chemical species can be considered to be major when its concentration (mole fraction) is higher than $1 \times 10^{-3}$. However significant differences are present for minor species (when concentration is lower than $1 \times 10^{-3}$ mole fraction) as it will be discussed in detail in Chapter 4 . 


\subsection{Confined Non-Premixed Swirl Burner}

The chemical reactor network was tested for a different burner set up in which the fuel and oxidizer were fed separately; non-premixed combustor. A swirl stabilized non-premixed methaneair burner, TECFLAM burner, was experimentally characterized by Schmittel et al. (Schmittel, et al. 2000). This burner was selected because of the large amount of experimental data available. The experimental data includes the following; temperature profiles, axial, radial, tangential velocity profiles, measured oxygen, water, carbon monoxide, and carbon dioxide concentration profiles.

\subsubsection{Geometry Details and Operational Conditions}

The TECFLAM burner has a water cooled combustion chamber with an internal diameter $D_{C}=0.5[\mathrm{~m}]$ and a height $L=1.2[\mathrm{~m}]$. The combustion chamber has two inlets Figure 3.14, the fuel annulus has an inner diameter of $D_{i, f u e l}=0.02[\mathrm{~m}]$ and outer diameter of $D_{o, f u e l}=0.026[\mathrm{~m}]$, the air annulus is defined by inner diameter of $D_{i, \text { air }}=0.03[\mathrm{~m}]$ and outer diameter of $D_{o, \text { air }}=0.06[\mathrm{~m}]$. The tangential velocity component was imposed by a swirler located upstream of the dump plane of the combustion chamber. The chamber has a bluff body at the center $D_{\text {bluff }}=0.02[\mathrm{~m}]$ which helps produce a large scale recirculation zone located at the center of the chamber. The recirculation helps to improve the fuel-air mixing process and results in a shorter flame. 


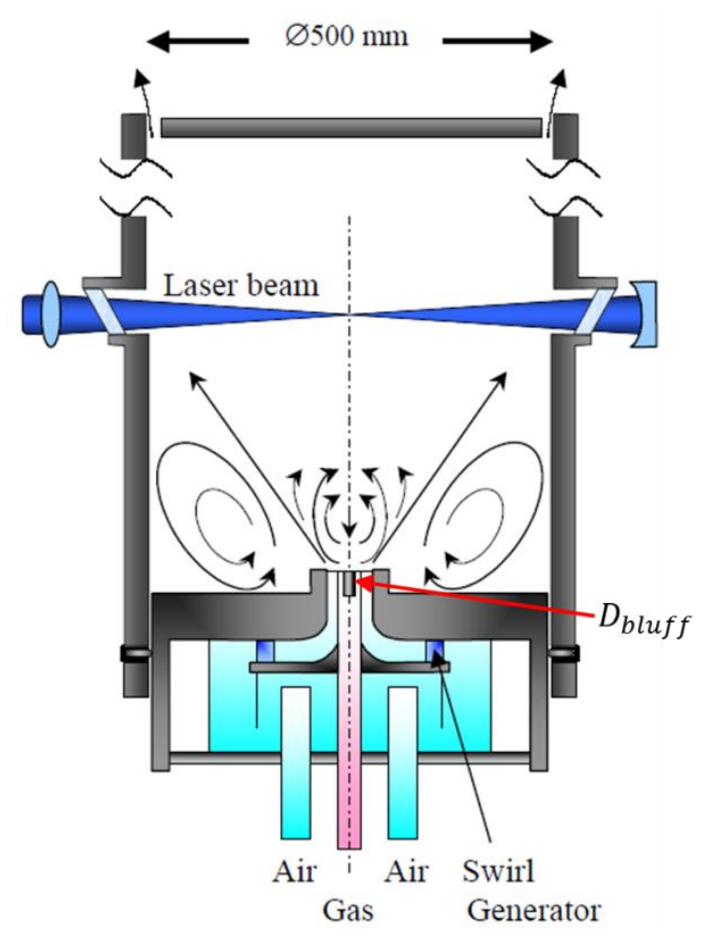

Figure 3.14. Schematic of the TECFLAM Burner (Meier, et al. 2000).

The TECFLAM burner has two main recirculation zones as it can be seen in Figure 3.14. A main recirculation zone is located at the center of the domain and is mainly generated by the sudden expansion of the flow. The tangential component of the flow is imposed upstream the dump plane by a swirl generator. The side walls are water cooled. Table 3.2 summarizes the burner geometry and the operational conditions used in this study.

Table 3.2. TECFLAM Geometry and Operational Conditions.

\begin{tabular}{|c|c|c|}
\hline Fuel Annulus & $D_{\text {inner }}=0.02[\mathrm{~m}]$ & $D_{\text {outer }}=0.026[\mathrm{~m}]$ \\
\hline Air Annulus & $D_{\text {inner }}=0.03[\mathrm{~m}]$ & $D_{\text {outer }}=0.06[\mathrm{~m}]$ \\
\hline Combustor Chamber Annulus & $D_{\text {inner }}=0.47[\mathrm{~m}]$ & $D_{\text {outer }}=0.5[\mathrm{~m}]$ \\
\hline Combustor Chamber Length, $L$ & $1.2[\mathrm{~m}]$ & \\
\hline Fuel Inlet Bulk Velocity & $21[\mathrm{~m} / \mathrm{s}]$ & \\
\hline Air Inlet Bulk Velocity & $23[\mathrm{~m} / \mathrm{s}]$ & \\
\hline Equivalence Ratio & 0.833 & \\
\hline Swirl Number, $S$ & 0.9 & \\
\hline Pressure & $1[\mathrm{~atm}]$ & \\
\hline Inlet Temperature & $300[\mathrm{~K}]$ & \\
\hline
\end{tabular}




\subsubsection{Simulation Details}

Two dimensional axisymmetric simulations were performed using ANSYS-FLUENT. The computational domain consisted of 71,428 cells. The computational domain used is shown in Figure 3.15 .
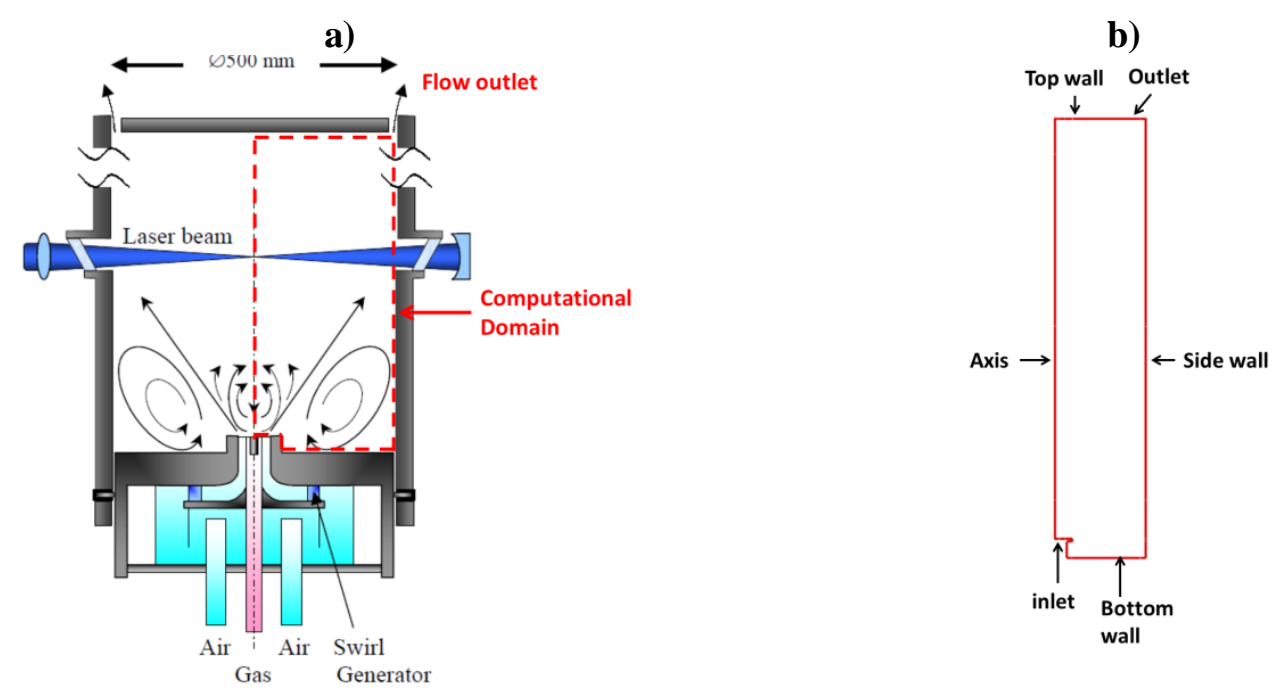

Figure 3.15. a) Sketch of the TECFLAM Burner, b) TECFLAM Computational Domain.

The dashed red line showed in Figure 3.15 a) represents the physical domain that was used in the CFD simulation. Figure 3.15 b) shows the simulated computational domain, here the inlet section has two annulus, the inner and outer annulus which supplied fuel and air respectively. Velocity inlet profiles from experiments were prescribed at the fuel and air inlets. The bottom and top wall were treated as adiabatic and no-slip boundaries. Side wall was set as a constant temperature $\left(T_{\text {wall }}=1073[\mathrm{~K}]\right)$, no-slip boundary. The outlet was set as an outflow boundary condition, finally the center line was set as an axis boundary condition. 
Table 3.3. Confined Non-Premixed Swirl Burner Simulation Details.

\begin{tabular}{|c|c|}
\hline & Reacting Flow Simulation \\
\hline Computational Domain & Two Dimensional Axisymmetric \\
\hline Time Formulation & Steady State \\
\hline Pressure-Velocity Coupling & SIMPLE \\
\hline Pressure Discretization Scheme & PRESTO \\
\hline Convective Term Discretization Scheme & $k$-epsilon \\
\hline Turbulence Model & Two Step Global Mechanism \\
\hline Chemical Mechanism & Finite Rate/Eddy-Dissipation \\
\hline Turbulence Chemistry Interaction Model & ISAT \\
\hline Chemical Species Integration &
\end{tabular}

The prediction of the flow field is highly sensitive to the shape of the inlet velocity profiles.

Previous studies ((Frassoldati, et al. 2005), (Chigier and Beer 1964)) and also the experience obtained in this study tells that the size and shape of the recirculation regions are greatly influenced by the shape of the inlet velocity profiles. A swirl number with the same inlet mass flow can be achieved by using different shapes of the velocity profiles but the predicted flow field could be very different.

In order to accurately predict the flow field, the velocity profiles used at the inlet of the domain are taken from experiments $1[\mathrm{~mm}]$ downstream of the dump plane. Cold flow simulations were not performed for this burner because of the availability of experimentally measured inlet velocity profiles. The inlet velocity profiles are shown in Figure 3.16.

a)

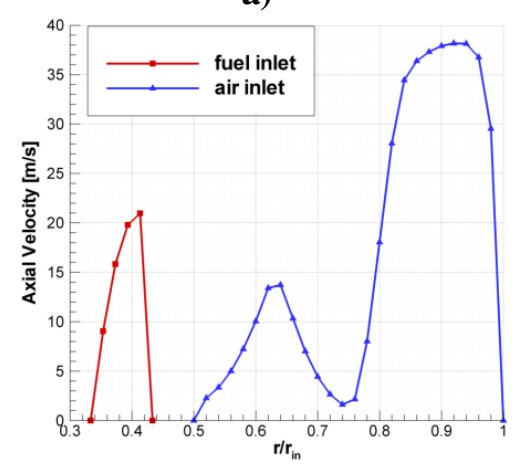

b)

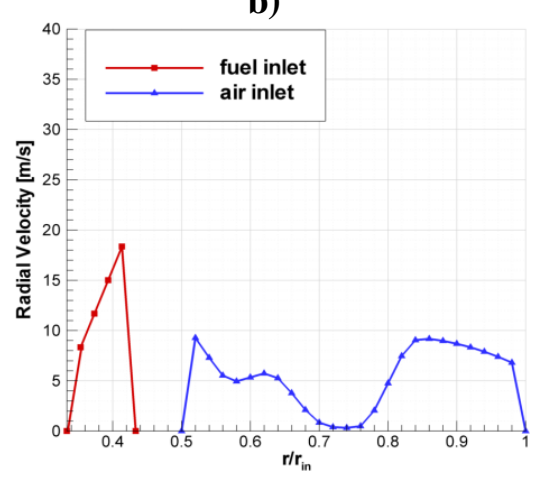

c)

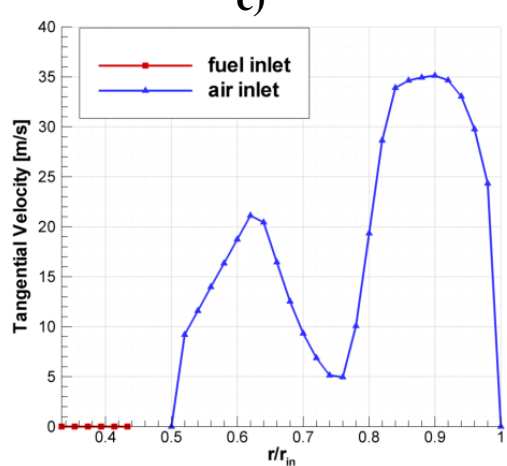

Figure 3.16. Measured Velocity Profiles at the Inlet of the TECFLAM Burner (Landenfeld, et al. 1998). a) Axial, b) Radial, and c) Tangential Velocity Components. 
The turbulence quantities were set using the turbulence intensity and the hydraulic diameter $(8 \%, 30[\mathrm{~mm}]$ for air inlet, $5 \%$ and $6[\mathrm{~mm}]$ for fuel). These values were adapted from Frassoldati (Frassoldati, et al. 2005).

\subsubsection{CFD Results for Reacting Flow - TECFLAM Burner}

Due the high non-linear nature of the problem a special solution strategy was followed to ignite the fuel-air mixture. The solution strategy is described next; 1) a numerical simulation of the non-reacting (isothermal) mixture was performed in order to obtain the major flow structures (such as the main recirculation zone), 2) chemical reactions were activated and the temperature of the gas inlet was set to a high value $\left(T_{\text {fuel,air }}=1500[\mathrm{~K}]\right)$ at this high temperature the chemical reactions were set in motion, 3) the gas inlet temperature was decreased until the experimental value was reached $\left(T_{\text {fuel,air }}=300[\mathrm{~K}]\right)$ taking attention to prevent the flame from extinguishing. It was not possible to ignite the mixture if this procedure was not followed. Once the temperature of the gas inlets was $300[\mathrm{~K}]$ the simulation was considered converged until the scaled residuals of the governing equations reached a minimum value of $1 \times 10^{-6}$, and the monitors of the axial velocity, mass fraction of carbon monoxide, and temperature measured close to the dump-plane and the outlet of the domain did not change significatively (less than $1 \%$ change with respect the value of the same quantity from the previous iteration). A detailed explanation of the scaled residuals calculation can be found in the section 28.15.1.1 in the FLUENT's users guide (ANSYS-FLUENT, User's Guide 2013).

Simulation results were compared with experimental data measured by (Landenfeld, et al. 1998), (Bockle, Kazenwadel and Schulz 2000). Natural gas was used as fuel in the experiments but in the current simulations it was approximated as $96 \%$ (vol) $\mathrm{CH}_{4}, 1.8 \% \mathrm{CO}_{2}$, and $\mathrm{N}_{2}$ as balance (Frassoldati, et al. 2005). 
a)

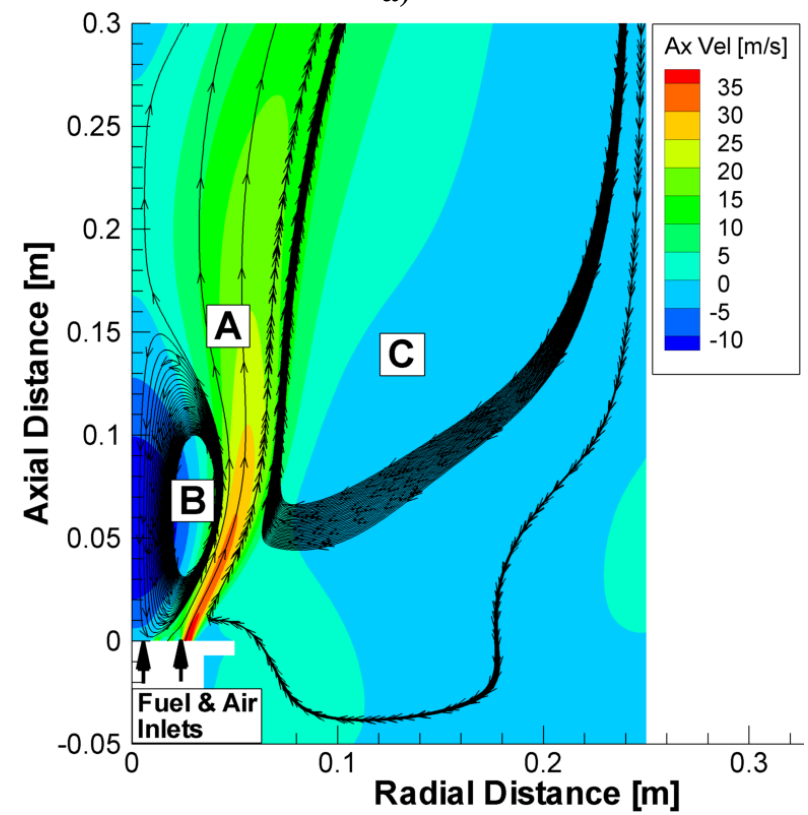

b)

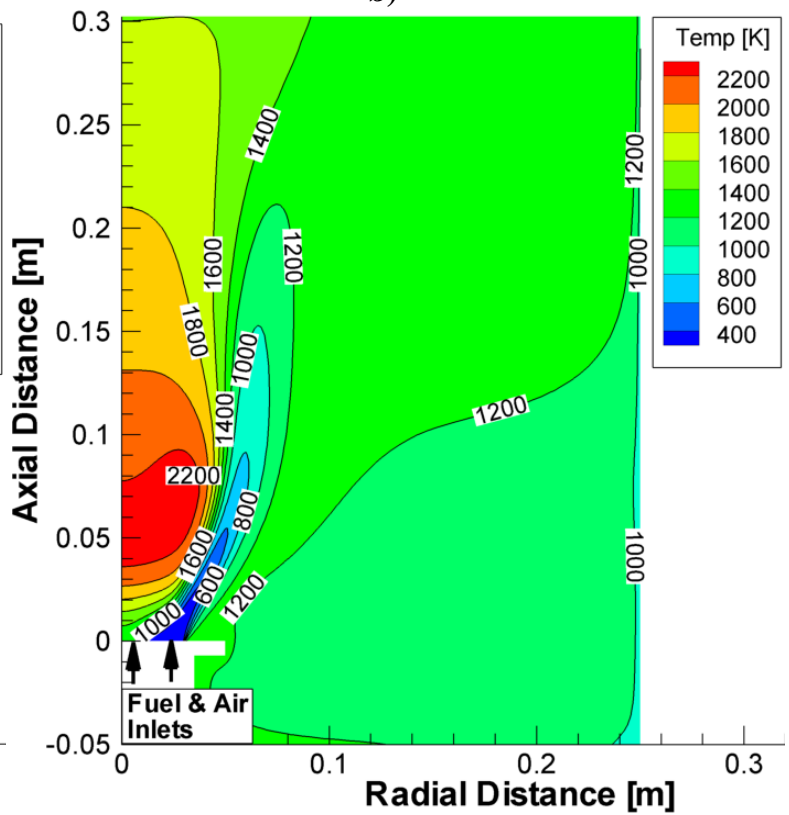

Figure 3.17. TECFLAM Burner Simulation Contours, a) Axial Velocity and Streamlines, b) Temperature.

Numerical predictions of the axial velocity for a region near the gas inlet are shown in Figure 3.17 a). The velocity field has the expected features for a flow with a swirl number $S>0.6$; flows with large swirl numbers have a main central recirculation zone (B) which can be detected by the negative values of the axial velocity, a mixing zone (A), and for confined flows there is a secondary recirculation zone (C) near the wall. The predicted flame has the characteristic tulip shape of swirl stabilized flames with vortex breakdown as shown in Figure $3.17 \mathrm{~b}$ ). The main central recirculation zone has the highest temperature of the domain because it is here where the combustion products recirculate and the combustion process is completed; exothermic reactions mainly take place at the mixing zone (A), and the secondary recirculation zone $(\mathrm{C})$ is composed of burnt products. The temperature in this region depends on the thermal boundary condition prescribed at the wall. 

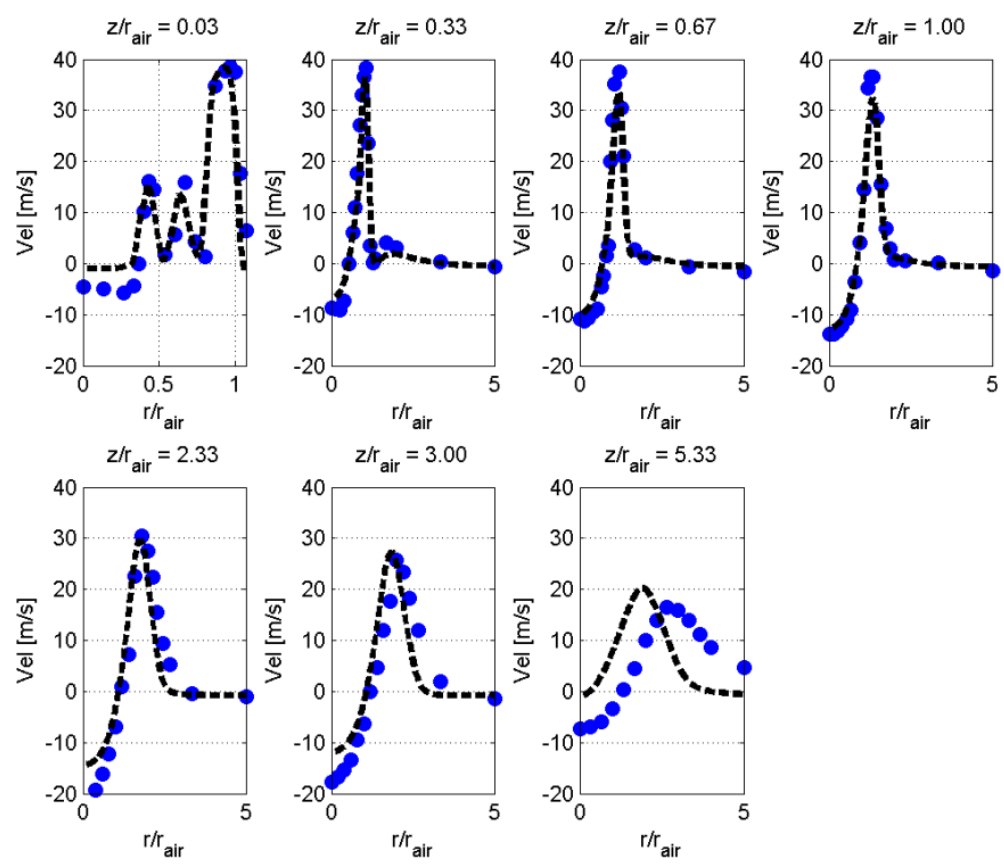

Figure 3.18. Axial Velocity Profiles. Circles - Experiments, Dash line - CFD Simulations.
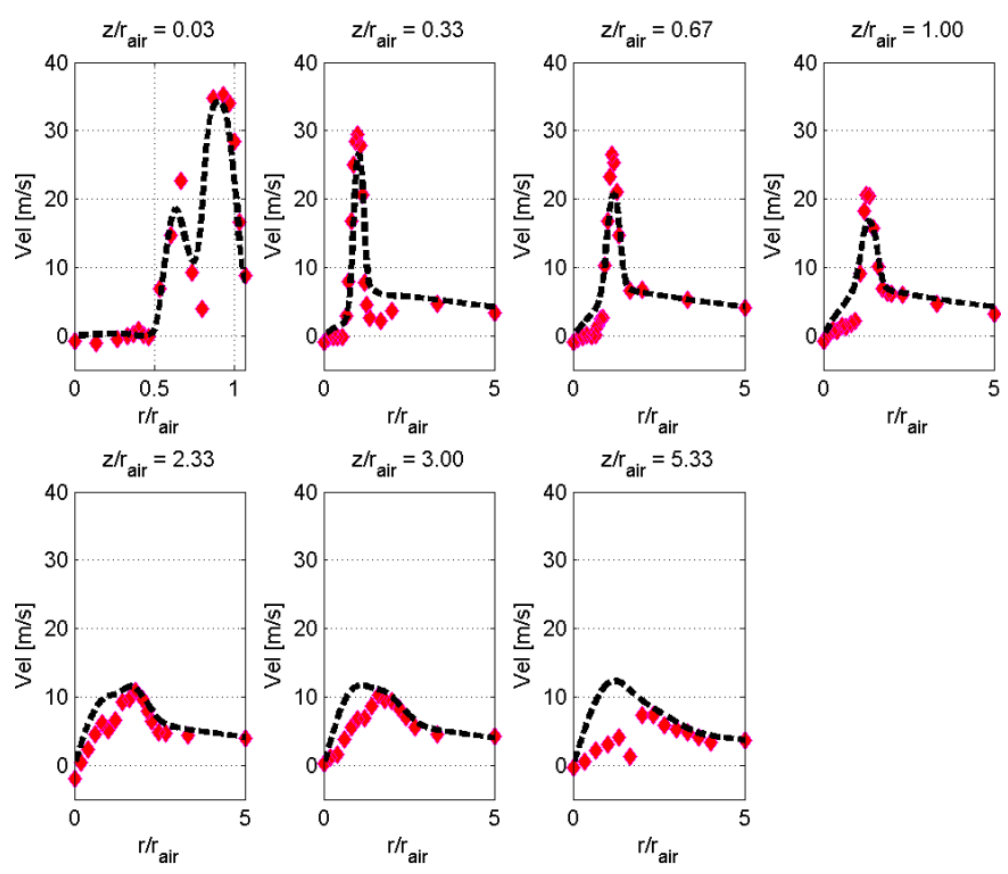

Figure 3.19. Tangential Velocity Profiles. Diamonds - Experiments, Dash line - CFD Simulations. 

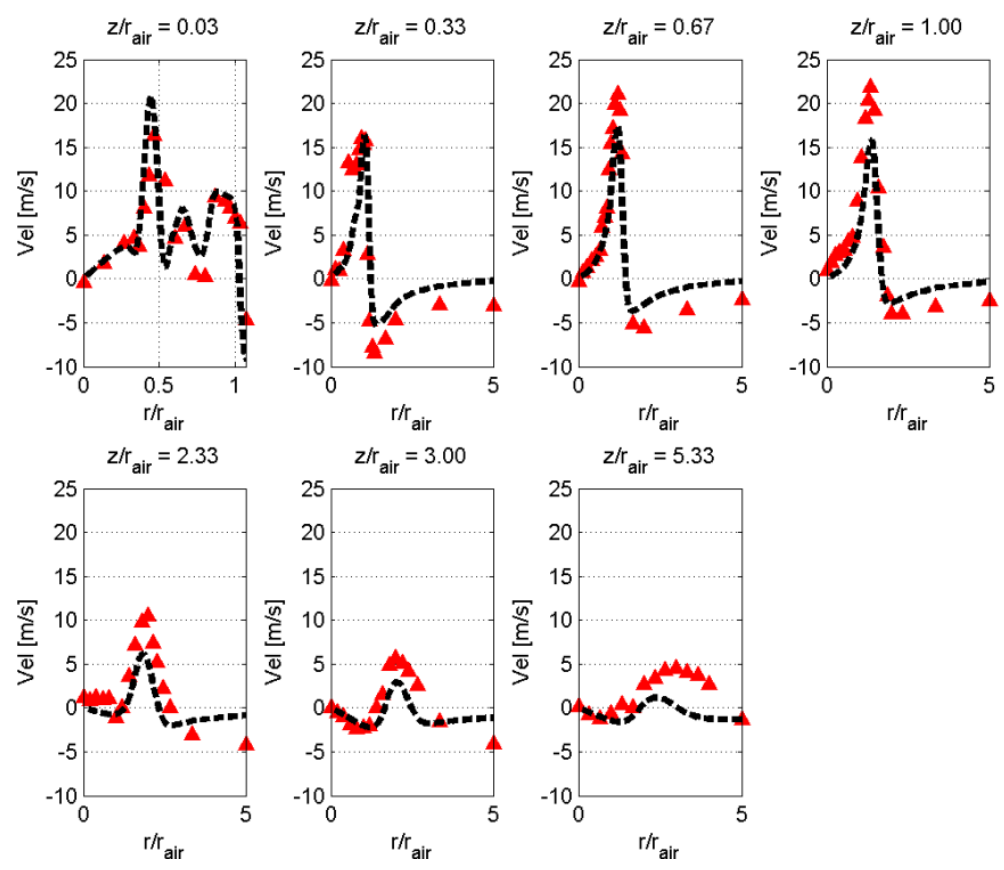

Figure 3.20. Radial Velocity Profiles. Triangles - Experiments, Dash line - CFD Simulations.

Validation of the predicted velocity field was performed using the data obtained from the Laser Doppler Velocimetry (LDV) measurements of the mean velocity components (Landenfeld, et al. 1998). Axial, tangential, and radial velocity components were measured along the radial direction at different stations located in the streamwise direction of the flow. An estimate of the statistical error concerning the mean velocity values was reported to be 5\% (Landenfeld, et al. 1998). In the present study the radial direction was normalized with respect the outer radius of the air annulus inlet $\left(r_{\text {air }}=0.03[\mathrm{~m}]\right)$.

Axial velocity profiles are shown in Figure 3.18, the first station located at $1[\mathrm{~mm}]\left(z / r_{\text {air }}=\right.$ 0.03 ) from the dump plane shows negative axial velocity values that indicate the presence of the central recirculation zone for both experiments and numerical simulations but the experiments indicate that near the axis $\left(r / r_{a i r}=0\right)$ the recirculation is stronger (more negative) than predicted results. The next three stations $\left(z / r_{\text {air }}=0.33,0.67\right.$, and 1.00$)$ showed a better match near the axis but the difference between the experiments and simulation increase again further downstream and 
the last station shows the maximum value from experiments is shifted to the axis as compared to predictions.

Predicted tangential velocity profiles (Figure 3.19) also follow the trend in the measured values. There is a difference in the maximum value of the tangential velocity from the second to the last station but the values near the axis matched the experimental values closely. In the same fashion as in the axial velocity profile, the maximum of the predicted profile is shifted towards the axis.

Radial velocity profiles shown in Figure 3.20 closely match the experimental values near the axis of the domain, and then further downstream the numerical simulations underestimate the maximum value of the radial velocity. The largest difference is seen at the last downstream station.

Simulations show that the main characteristic of the profiles were captured and fairly good agreement was obtained with measurements, but there are differences in the peak values of the velocity profiles. This inaccuracy can in part be attributed to the utilization of the standard k-epsilon turbulence model that assumes isotropic behavior of the Reynolds stress tensor, which is generally not the case in swirled flames. A possible improvement to the simulations could be the implementation of a different turbulence model, e.g. the Reynolds Stress Model (RSM). The RSM does not assume the Reynolds stress tensor to be isotropic; the RSM solves a transport equation for each of the components of the tensor which for a two dimensional simulation means the addition of five extra partial differential equations. The implementation of the RSM turbulence model does not always yield results that are clearly superior to the simpler models (such as the standard k-epsilon) but the computational time is exponentially increased (ANSYS-FLUENT, Theory Guide 2009), see Appendix 3. The goal of the current simulations was to reduce the computational time needed to predict behavior of a combustor. For this reason the most inexpensive turbulence model that could give satisfactory predictions was employed. The standard k-epsilon model was used for the same 
experimental set up by Frassoldatti (Frassoldati, et al. 2005) and the predicted results matched the experimental measurements fairly well.
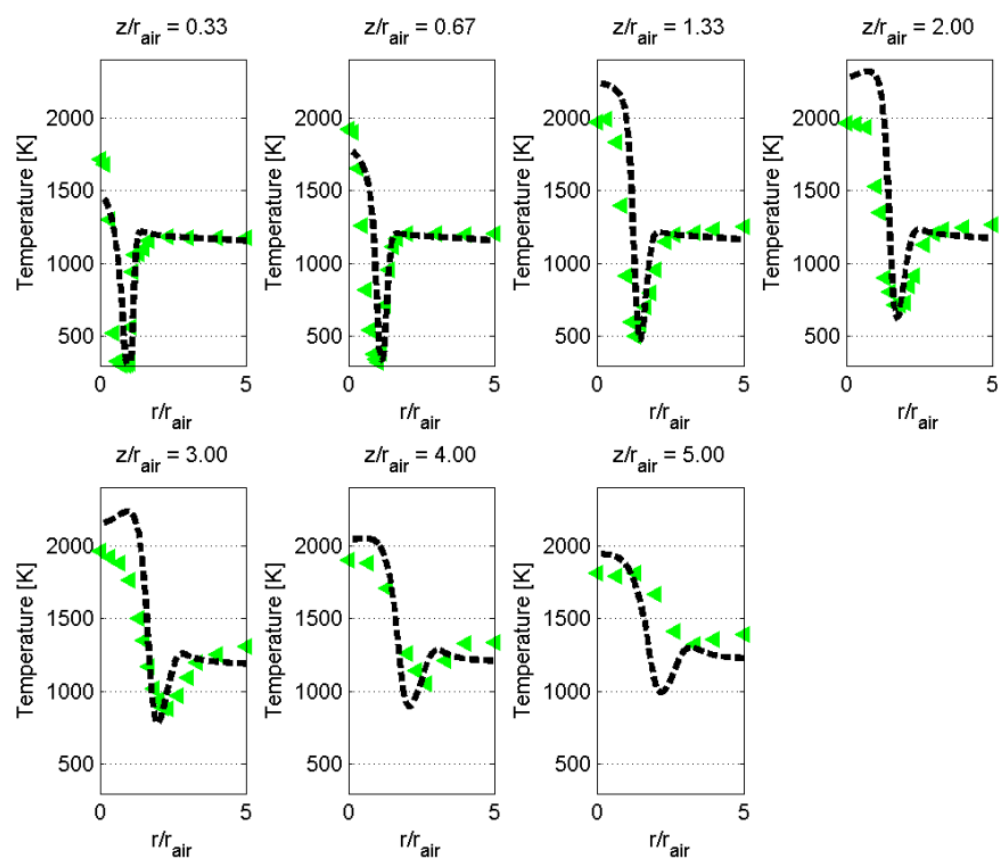

Figure 3.21. Temperature Profiles. Left Triangles - Experiments, Dash line - CFD Simulations.

Figure 3.21 shows the measurements of the mean temperature, the accuracy achieved for the mean temperature measurements was reported to be between 2-3\% (Meier, et al. 2000). Numerical simulations were able to capture the temperature trends that were measured in experiments (Figure 3.21). The calculated temperature profiles showed a hotter region near the axis with a colder section immediately after the axis (moving in the radial direction), this cold region is produced by the fresh incoming mixture. The third region that forms in the radial direction for all the stations depicts the secondary recirculation zone, this region is not as hot as the main recirculation zone because it is affected by the cold wall $\left(T_{\text {wall }}=1073[\mathrm{~K}]\right)$. Temperature predictions showed that near to the dump plane $\left(z / r_{\text {air }}=0.33\right.$, and 0.67$)$ the calculated temperature close to the axis is lower than experiments but the predictions match the experiments away from the symmetry axis. However, numerical predictions have higher temperatures compared to experiments 
for the rest of the stations further downstream. The discrepancies in temperature calculations can be partially attributed to the simplifications introduced by the chemical kinetic model used. Two step reaction mechanism influences the final temperature results mainly because the model neglects some dissociation reactions that might reduce the temperature due to its endothermic nature. The only dissociation reaction included in the model is the carbon dioxide decomposition into carbon monoxide and oxygen. The chemical mechanisms used in the present study are shown in the Appendix 1 section.
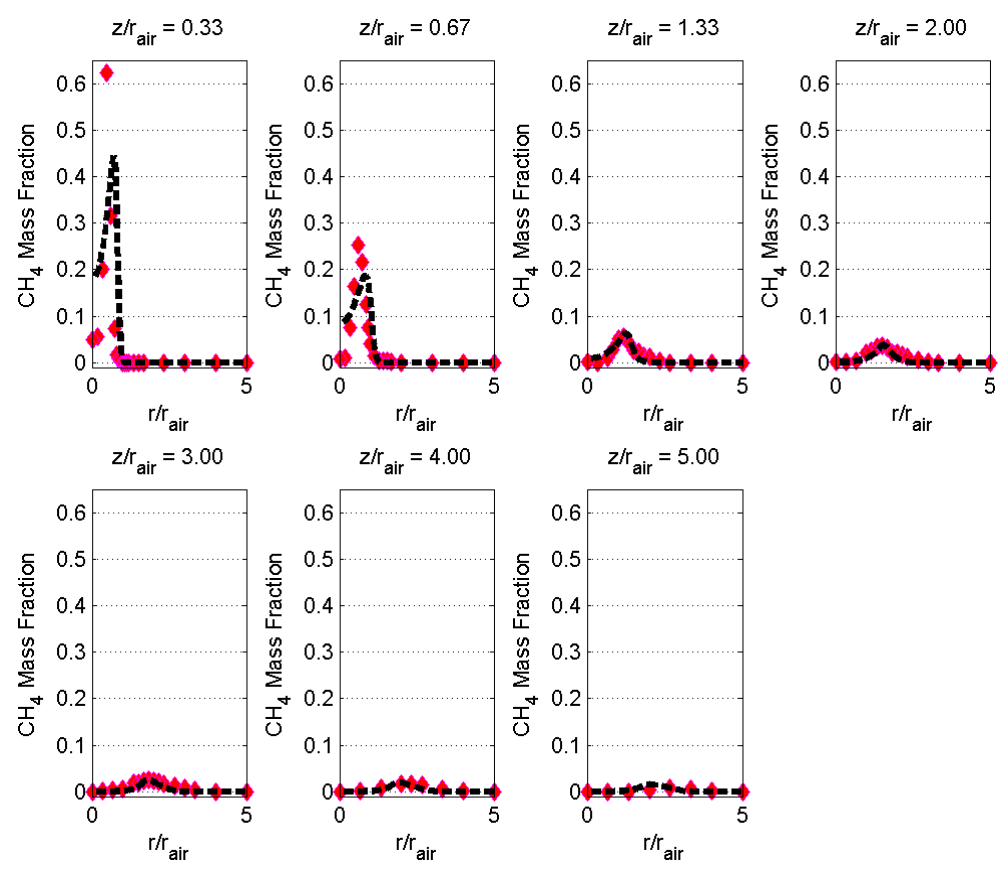

Figure 3.22. Methane Mass Fraction Profiles. Diamonds - Experiments, Dash line - CFD Simulations.

Mass fraction measurements were reported by Meier (Meier, et al. 2000), Meier reported an accuracy of the mean values of nitrogen to be $2 \%, 4 \%$ for carbon dioxide, and a smaller accuracy for the rest of the species (values were not reported for the other species). However, Meier reported the accuracy for the single pulse Raman measurements of the water mass fraction to be 5-7\%, 12$15 \%$ for oxygen, $2 \%$ for methane, and $20 \%$ for carbon monoxide when the mole fraction was near 
0.02 and $50 \%$ when the mole fraction was near to 0.02 . According to the authors the accuracy of the mass fraction mean values is expected to be higher than the single pulse Raman measurements.

Mass fraction profiles of methane are shown in Figure 3.22. Predicted methane mass fraction near the dump plane $\left(z / r_{\text {air }}=0.33\right.$, and 0.67$)$ shows lower values than the recorded experimental measurements. The predictions overestimated mixing near the dump plane, methane appears to be "diluted" with respect the concentration measured in the experiments. The agreement between the predictions and the experiments improves further downstream. On the other hand, the predicted mass fraction of oxygen (Figure 3.23) matches the experiments near the axis with the maximum mass fraction located at the same position as the experiments. Predictions deviate from the experiments as the mixture moves downstream and a large noticeable discrepancy in the results is seen at the last station $\left(z / r_{a i r}=5.0\right)$.
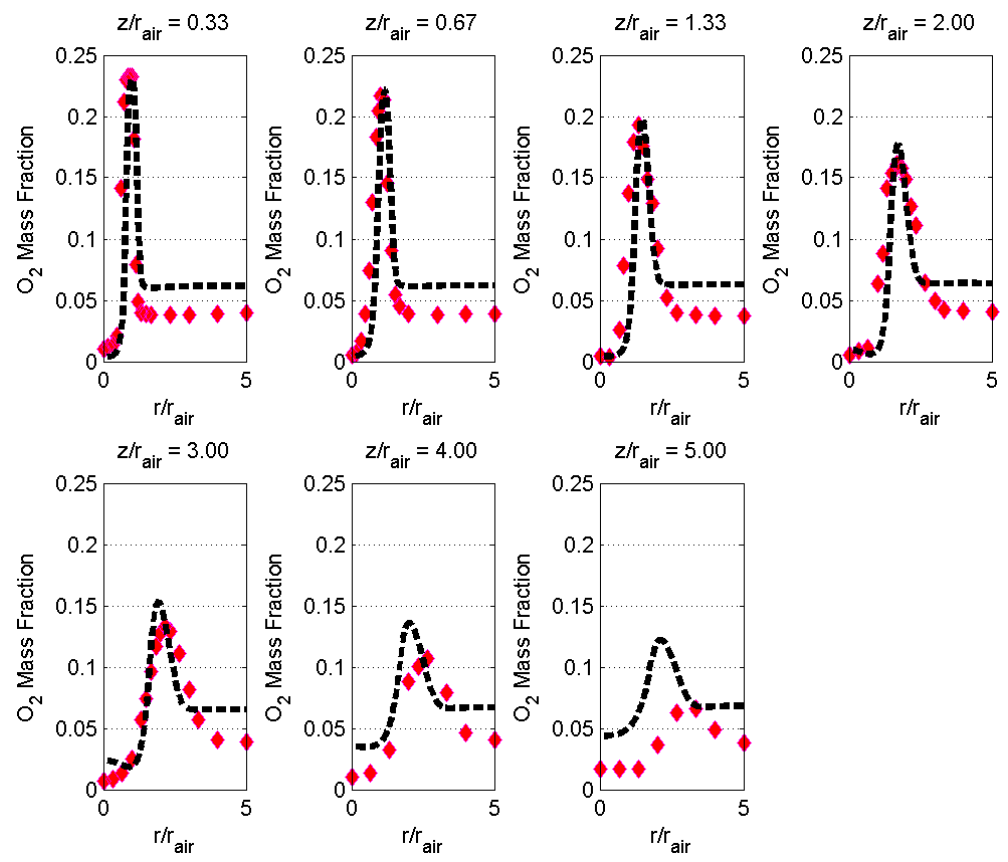

Figure 3.23. Oxygen Mass Fraction Profiles. Diamonds - Experiments, Dash line - CFD Simulations. 

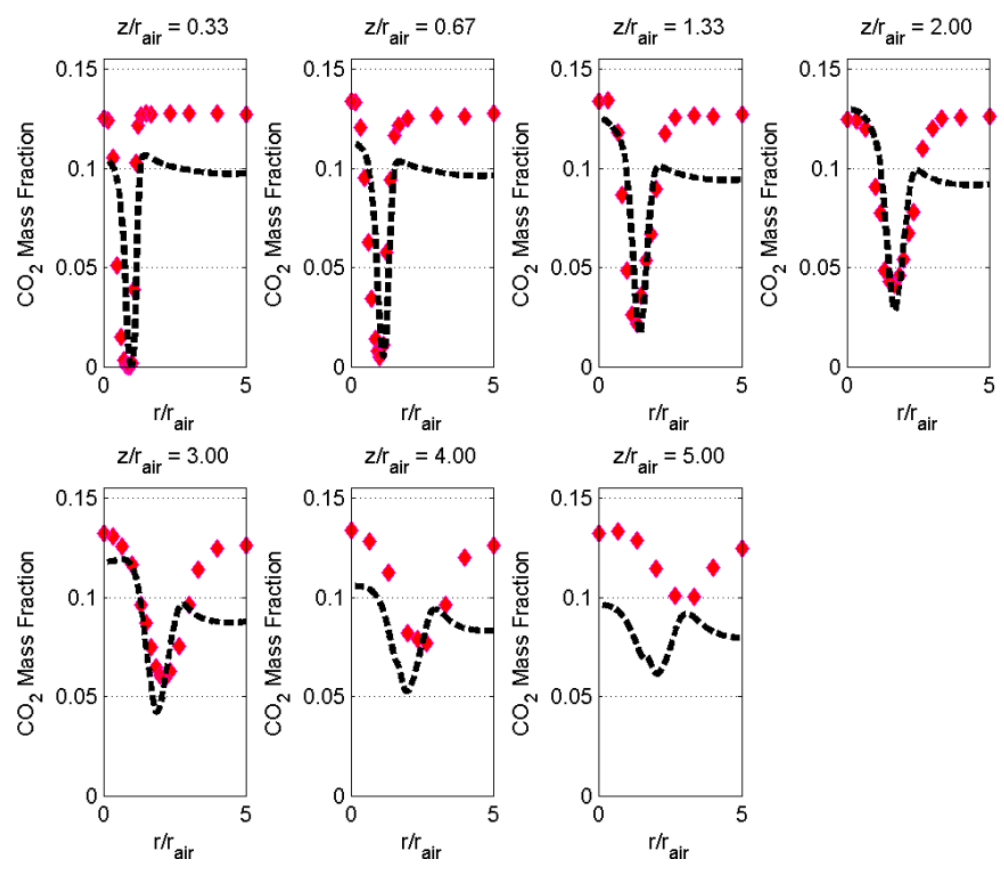

Figure 3.24. Carbon Dioxide Mass Fraction Profiles. Diamonds - Experiments, Dash line - CFD Simulations.
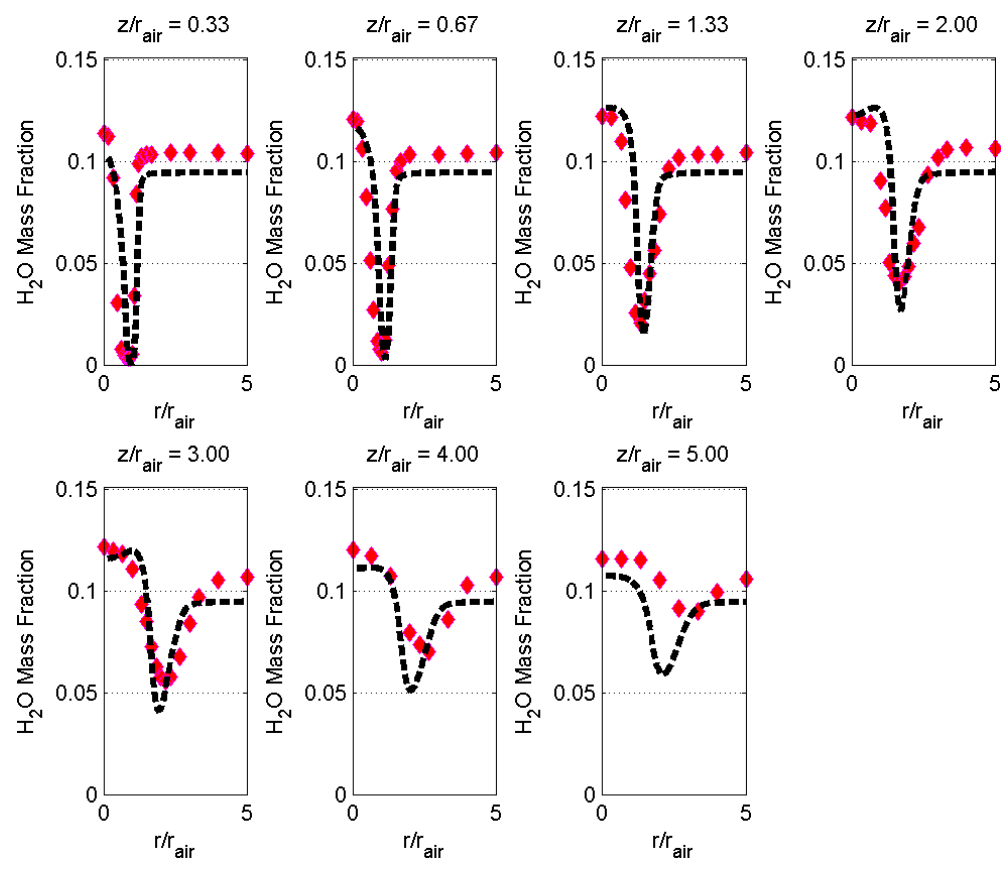

Figure 3.25. Water Vapor Mass Fraction Profiles. Diamonds - Experiments, Dash line - CFD Simulations. 
Carbon dioxide and water vapor mass fraction profiles (Figure 3.24, and 3.25) followed similar trends as the temperature profiles. The higher temperature near the axis means that the highest concentration for carbon dioxide and water was at this location because it was here where the combustion products recirculated and the combustion process was completed. The overall trend of the mass fractions of carbon dioxide and water is in agreement with experiments. There was a significant difference between the experiments and the simulations especially at the last measurement station.

\subsection{Unconfined Premixed Swirl Burner}

An unconfined premixed methane-air swirl stabilized burner was also studied. Being an unconfined geometry poses a challenge to implement appropriate boundary conditions that will allow entrainment of the surrounding fluid to the flame region. Predictions obtained using RANS were compared with experimental results that were previously reported in the literature. The experimental data of axial velocity contours, temperature, oxygen, carbon dioxide, and NO profiles were previously reported by Kim et al. (Kim, Arghode and Gupta 2009). The problem setup is discussed next along with the numerical simulations.

\subsubsection{Geometry Details and Operational Conditions}

The experimental set up for this burner is shown in Figure 3.26. A laboratory-scale unconfined premixed methane-air swirl stabilized burner was studied. The burner is open to the room and the inlet reactants enter to the swirler at room temperature. 


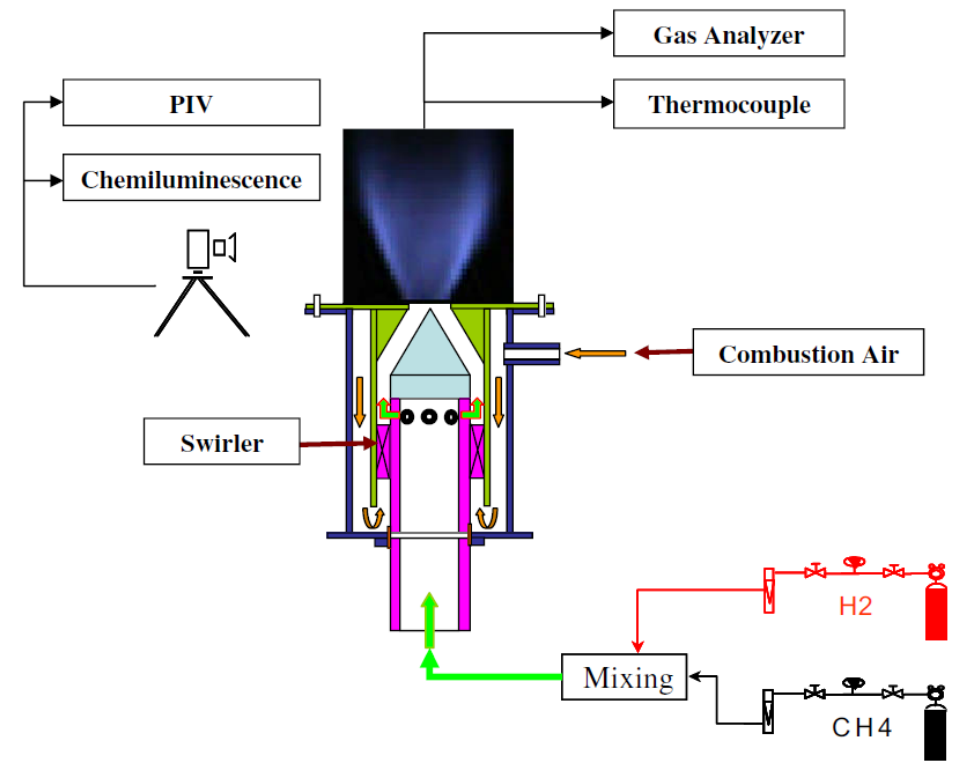

Figure 3.26. Experimental Lab-Scale Swirl Stabilized Burner (Kim, Arghode and Gupta 2009).

For the case studied in the present work only methane was used as fuel with an equivalence ratio of Eq.rat. $=0.717$. The swirl was applied by means of a coaxial swirler with a blade angle of $45[\mathrm{deg}]$ that produces a geometric swirl number of $0.67\left(S_{\text {geom }}=0.67\right)$. The gas exit located at the dump plane has a diameter of $D_{\text {dump }}=20$ [mm]. The mass inlet flow used corresponds to 142 [slpm] of air and the balance of methane that satisfies the equivalence ratio.

\subsubsection{Simulation Details}

A two dimensional sketch of the burner is shown in Figure 3.27. 


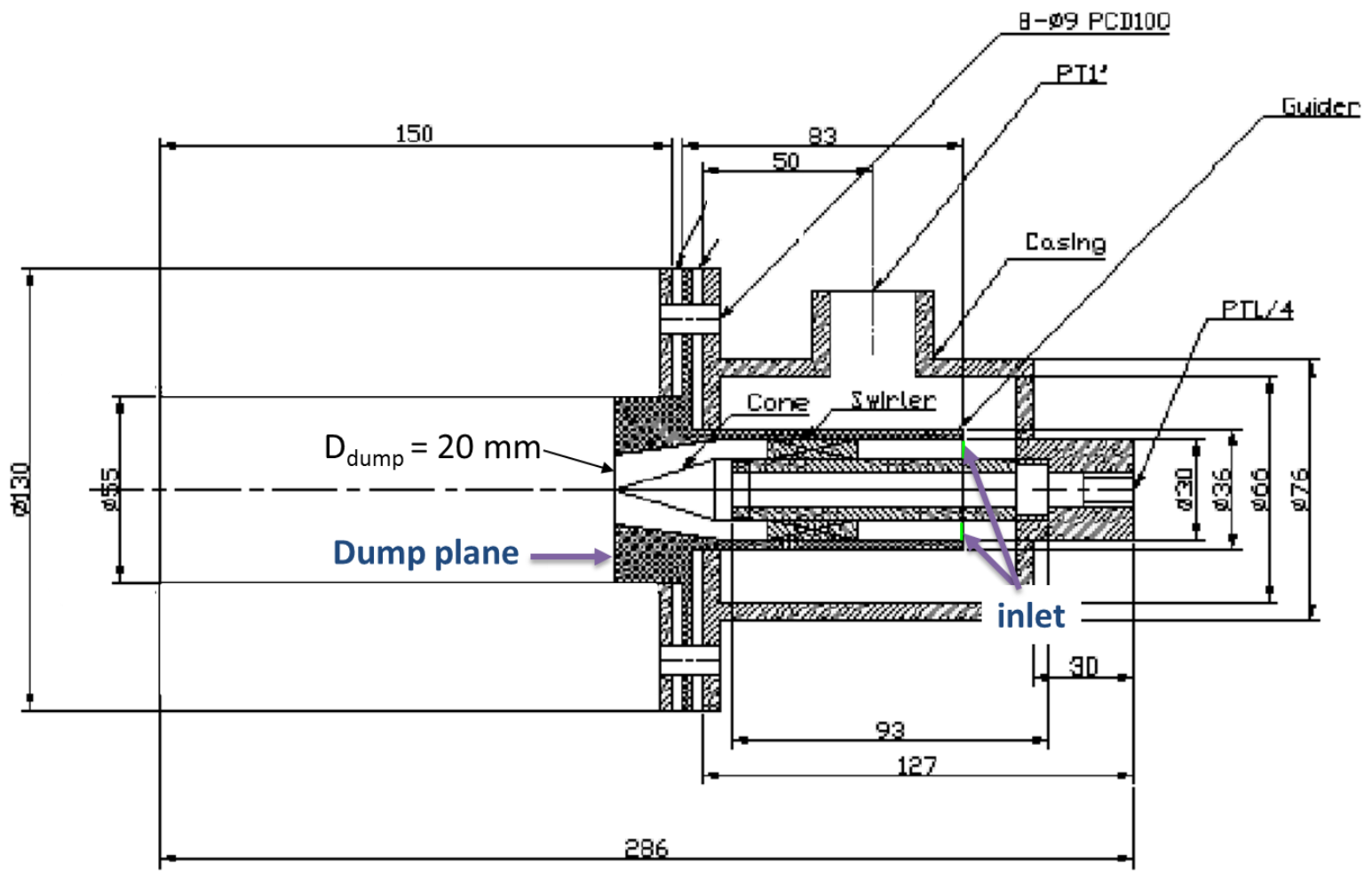

Figure 3.27. Two Dimensional Sketch of the Unconfined Swirl Stabilized Burner (Gupta and Khalil 2013).

Figure 3.27 shows the dimensions in $[\mathrm{mm}]$ of the unconfined swirl burner. The mixed reactants enter to the annular section marked as inlet in the Figure 3.27. Reactants flow through the blade swirler to enter a convergent section that is named as cone in the sketch. Finally the reactants are dumped into the room where they expand due to the tangential momentum $(S>0.6)$ that the flow carries. A negative pressure gradient in the axial direction is created producing a recirculation zone.

Several computational domains were used for the simulation of this burner:

1. Two dimensional axisymmetric domain with the lateral contour of the burner.

2. Three dimensional domain without the swirler.

3. Three dimensional domain with the swirler section included. 
a)

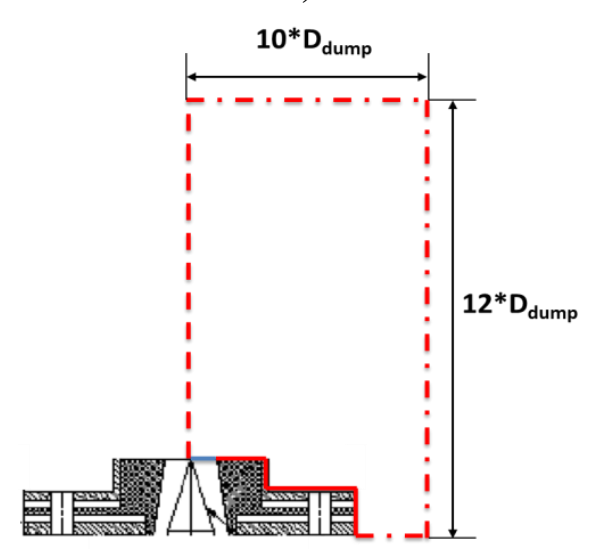

c)

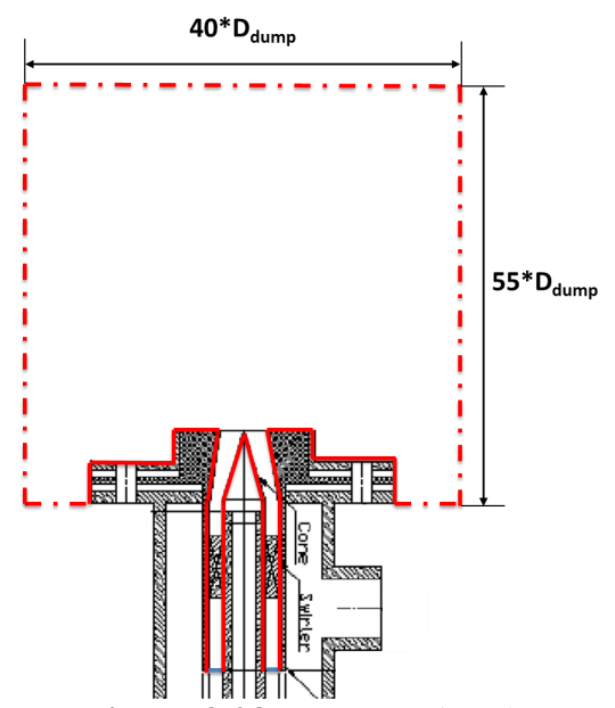

b)

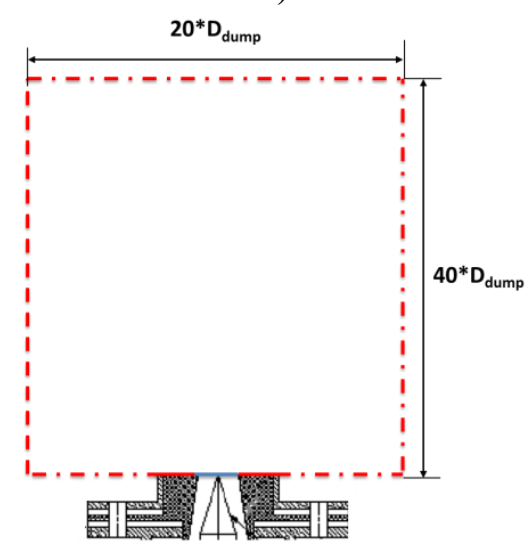

Figure 3.28. Computational Domains of the Unconfined Swirl Stabilized Burner.

Figure 3.28 shows different computational domains used to simulate the unconfined swirl burner configuration. The computational domains created started from the most simple geometry in which the swirler was not included and only a two dimensional axisymmetric domain was used, Figure 3.28 a). Predictions using the domain from Figure 3.28 a) did not produce satisfactory results because the flame thickness was not properly predicted, for this reason a three dimensional domain was considered. Figure 3.28 b) shows the dimensions used in the three dimensional simulation, the value of $20 * D_{\text {dump }}$ is the diameter dimension and the length of the channel is $40 * D_{\text {dump }}$. Three dimensional simulations slightly improved the results but they did not fairly match the experiments, the temperature difference between the CFD predictions and the experimental 
measurements was $42\left[{ }^{\circ} \mathrm{C}\right]$ near the axis for the first measuring station $(z=7.62[\mathrm{~mm}])$ but then the difference increased to $416\left[{ }^{\circ} \mathrm{C}\right]$ near the center for the last measuring station $(z=22.9[\mathrm{~mm}])$. Finally a three dimensional domain in which the inlet section was included in the simulation, the parts added in the simulation were the annular inlet, the swirler, and the convergent section located just before the dump plane. The reason why the simulations were not started from the complete three dimensional domain including the swirler was because the information about the geometry was not available in the paper that contains the experimental data (Kim, Arghode and Gupta 2009). Thanks to the kind help from Professor Ashwani Gupta and his graduate student Ahmed Khalil from the University of Maryland (Gupta and Khalil 2013) who provided a sketch with the dimensions of the inlet section and the details of the swirler, it was possible to simulate the entire burner. The boundary conditions employed for each of the simulated domains are shown in Figure 3.29 . 
a)

Pressure

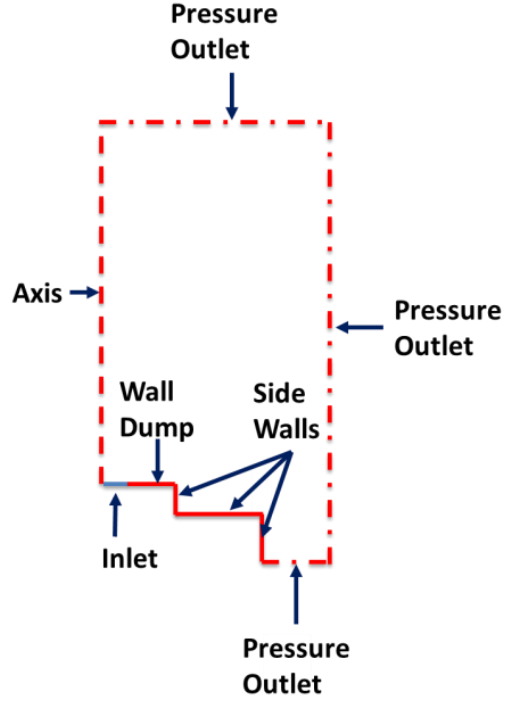

b)

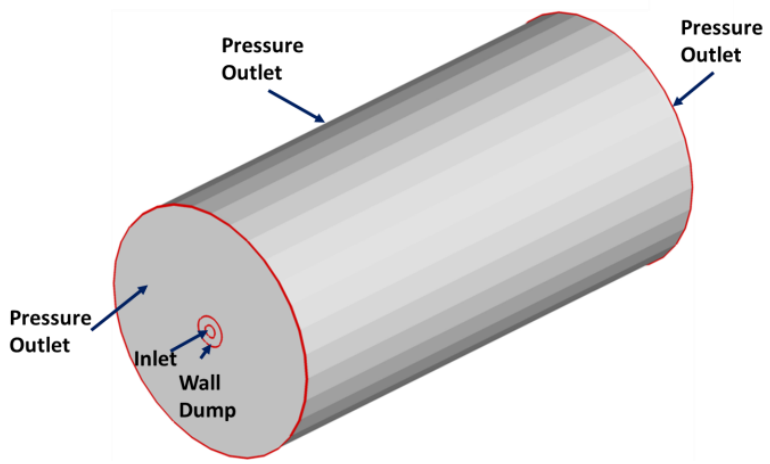

c)

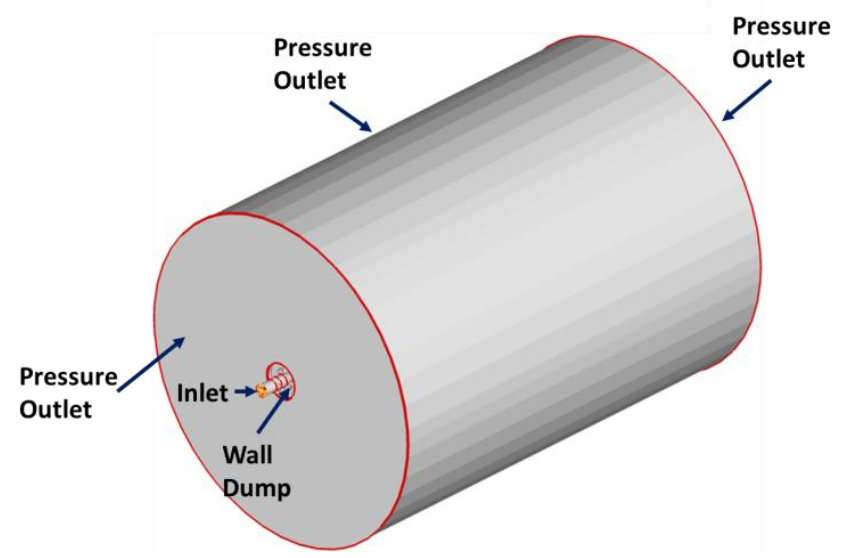

Figure 3.29. Boundary Conditions for the Unconfined Swirl Stabilized Burner.

Simulations were performed using uniform and non-uniform velocity profiles at the inlet, a detailed discussion of the shape of the velocity profiles will be given later in this chapter. A nonslip velocity boundary condition was prescribed at the region named Wall Dump, and Side Walls along with adiabatic wall condition. The two dimensional axisymmetric domain 3.29 a) included a line that was defined as the domain axis. It must be mentioned that the case shown in Figure 3.28 a) used the same boundary conditions depicted in Figure 3.29 a). Pressure outlet boundary conditions were used for the bottom, side, and top boundaries. Pressure outlet boundary condition allowed the entrainment of the surrounding fluid, if outflow boundary condition was used then no entrainment 
was present in the problem. This is basically because the outflow boundary condition extrapolates the velocity and pressure values from the interior of the domain but it always re-scales the velocity values in order to satisfy the mass balance of the problem. If incoming flow is going through the outflow boundary condition then the simulation becomes unstable unless the amount of entrained mass is specified beforehand which is not possible because it is not known a priori. The pressure was set equal to the atmospheric pressure of the room.

The swirler included in the three dimensional domain is shown in Figure $3.29 \mathrm{c}$ ), it was included in order to have a better calculation of the recirculation zone located downstream the dump plane. Figure 3.30 shows the details of the swirler.

a)

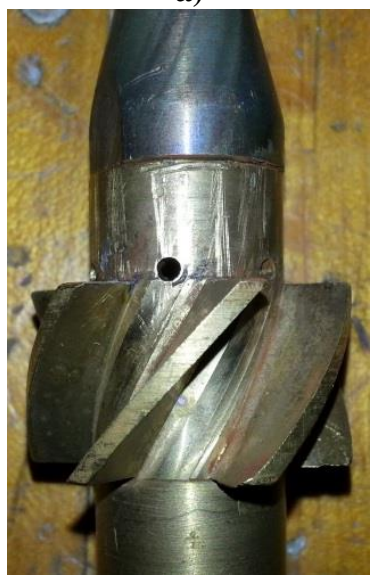

b)

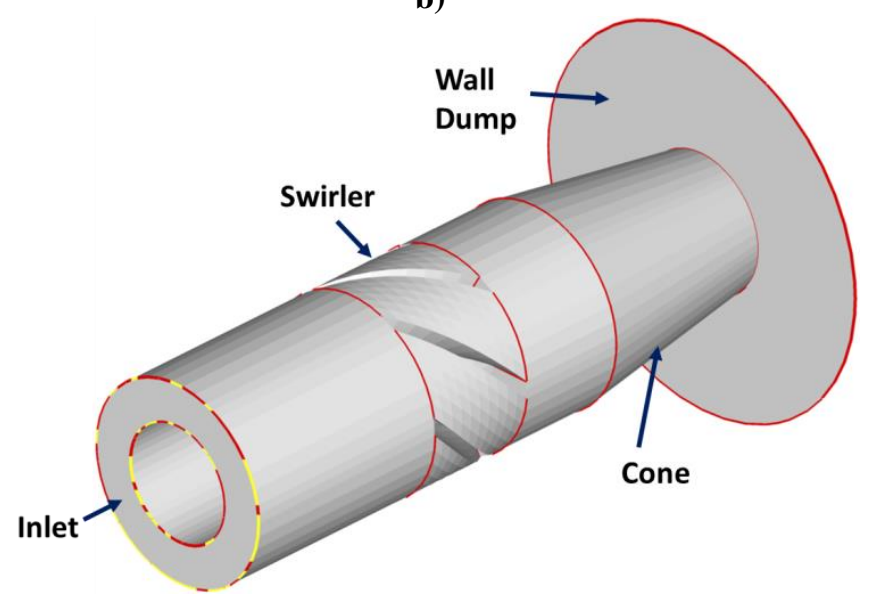

Figure 3.30. a) Swirler, b) Computational Domain of the Inlet.

Figure 3.30 a) is a picture of the 45 [deg] blade swirler. The swirler consists of; six blades, each blade with a thickness of $\sim 2.6[\mathrm{~mm}], D_{\text {outer, blade }}-D_{\text {inner,blade }}=12[\mathrm{~mm}]$, the length in the axial direction is $16.5[\mathrm{~mm}]$. The computational domain generated from the inlet section is shown in Figure $3.30 \mathrm{~b}$ ); here a uniform velocity profile was prescribed at the inlet surface. As it can be seen from Figure $3.30 \mathrm{~b}$ ) the swirler is included and downstream of the swirler there is a convergent section denoted as cone. 
When the initial simulations were performed no information of the velocity profiles at the dump plane was available, for this reason the shape of the velocity profiles was assumed. Uniform and non-uniform velocity profiles were specified at the dump plane (with the exception of the three dimensional domain that includes the swirler, the velocity profiles at the dump plane were calculated as part of the solution). The non-uniform velocity profiles were designed in such a way that the total mass coming into the domain matched the experiments and the tangential component was modified in order to satisfy the same swirl number as reported for the experiments.

The velocity values for the uniform velocity profiles are the following; $U_{\text {axial }}=7.8[\mathrm{~m} / \mathrm{s}], U_{\text {tangential }}$ $=7.8[\mathrm{~m} / \mathrm{s}]$. Non-uniform velocity profiles are shown next.

a)

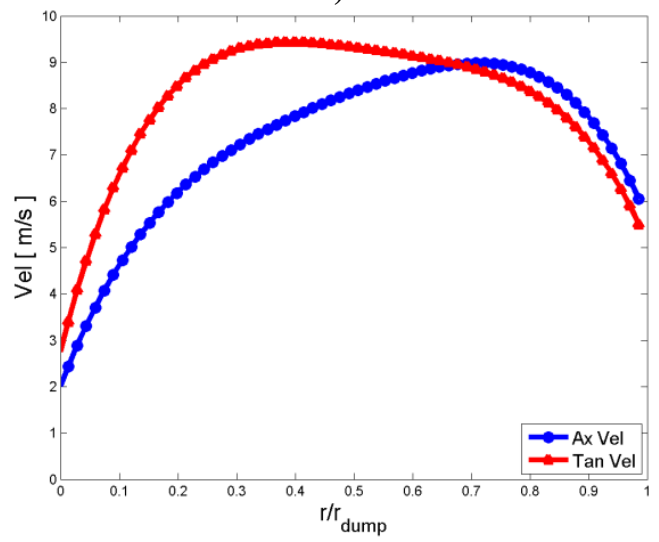

c)

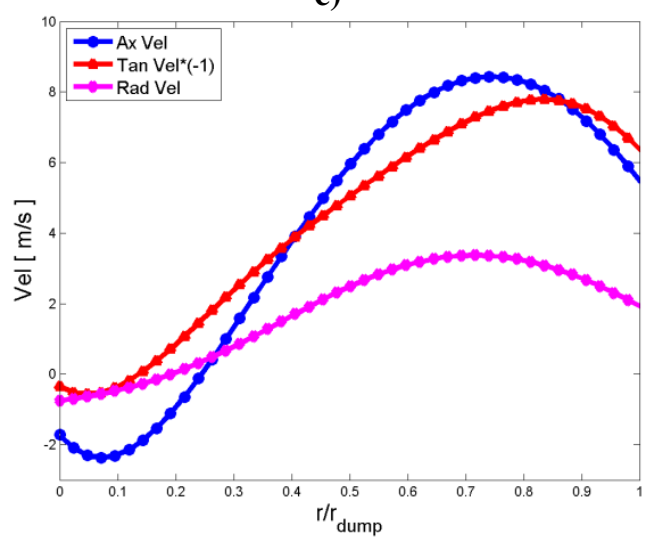

b)

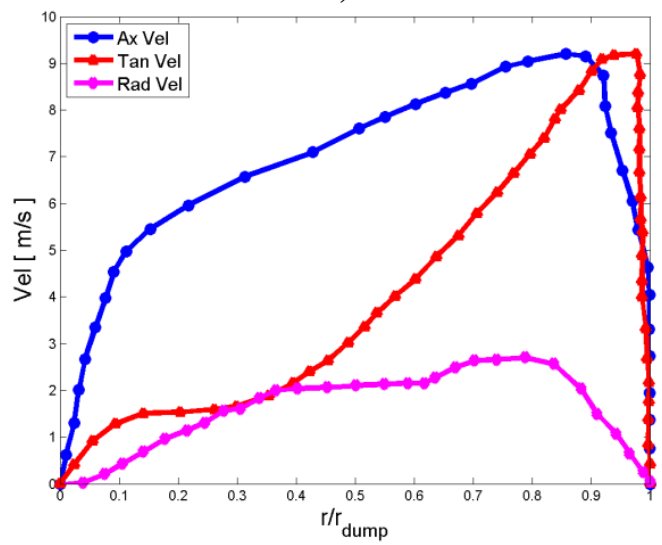

Figure 3.31. Non-Uniform Velocity Profiles. a) Scaled from NETL Burner, b) Scaled from Chigier and Beer (Chigier and Beer 1964), c) Experiments (Gupta and Khalil 2013). 
Profiles shown in Figure 3.31 a) were created using the shape of the profiles from Section 3.2 (NETL burner), the axial velocity profiles were scaled in order to match the same mass flow and the tangential velocity profiles to match the swirl number $\left(S_{\text {geom }}=0.67\right)$. No radial component was prescribed in this set up. Another set of profiles were assumed in order to include the effect of the convergent section that is located between the swirler and the dump plane (cone), Figure 3.31 b). A similar problem setup was reported by Chigier and Beer (Chigier and Beer 1964), using the reported shapes the profiles were scaled in order to satisfy the constraints of mass flow and swirl number. The radial velocity component was included following the profile shape reported by Chigier and Beer. Finally a set of measured profiles (Gupta and Khalil 2013) was used as the inlet boundary condition, Figure $3.31 \mathrm{c}$ ).

Table 3.4. Unconfined Premixed Swirl Burner Simulation Details.

\begin{tabular}{|c|c|}
\hline Computational Domain & Reacting Flow Simulation \\
\hline Time Formulation & $\begin{array}{c}\text { i) Two Dimensional Axisymmetric } \\
\text { ii) Three Dimensional }\end{array}$ \\
\hline Pressure-Velocity Coupling & Steady State \\
\hline Pressure Discretization Scheme & SIMPLE \\
\hline Convective Term Discretization Scheme & PRESTO \\
\hline Turbulence Model & $k$-epsilon $R N G$ \\
\hline Chemical Mechanism & a) One Step Global Mechanism \\
& b) ARM9 \\
\hline Turbulence Chemistry Interaction Model & Eddy-Dissipation Concept (EDC) \\
\hline Chemical Species Integration & ISAT \\
\hline
\end{tabular}

Table 3.4 shows more details on the numerical model used in the simulations. The three dimensional domain corresponds to the cases shown in $3.28 \mathrm{~b}$ ), and c) and $3.29 \mathrm{~b}$ ) and c). Several more case simulations were performed but only the most relevant are reported in Table 3.4.

\subsubsection{CFD Results - Unconfined Premixed Swirl Burner}

Non-reacting flow simulations were performed using non-uniform profiles scaled from the results obtained from the NETL burner. The numerical domain consisted of 85,513 non-uniform cells, mesh resolution concentrated in the region where the flame and the recirculation region are 
located. Results are compared with measured PIV data (Gupta and Khalil 2013). Results are shown in Figure 3.32.

a)

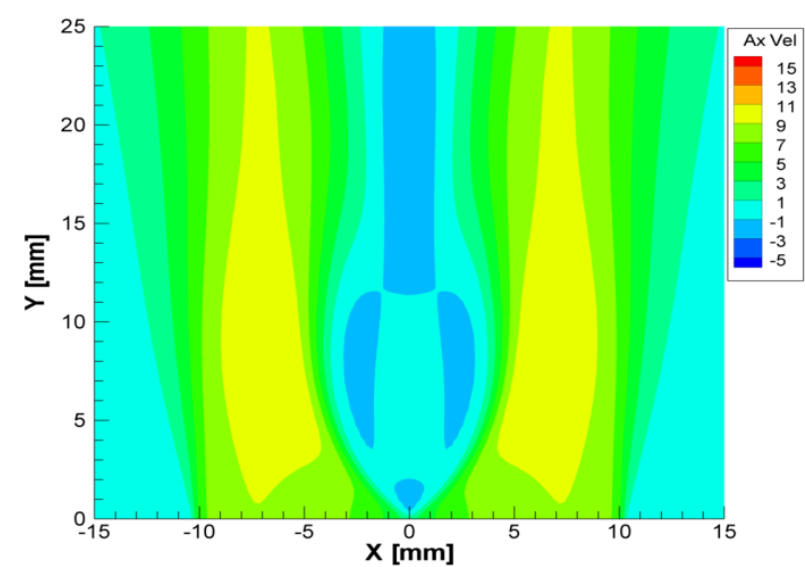

b)

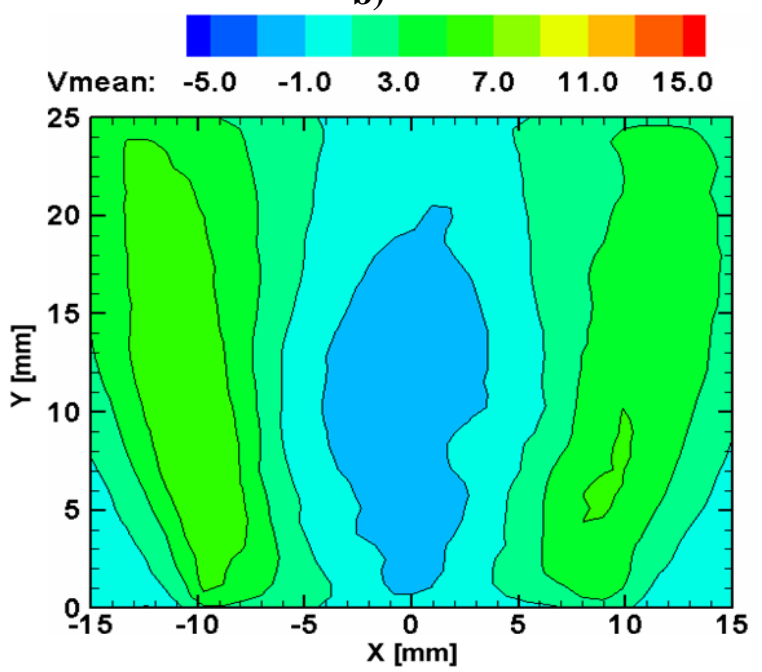

Figure 3.32. Axial Velocity [m/s] Contours Non-Reacting Flow. a) Two Dimensional Axisymmetric with NETL Scaled Velocity Profiles, b) Experiments (Gupta and Khalil 2013).

In Figure 3.32 the $X$-direction represents the radial direction in Cartesian coordinates and the $Y$-direction is the equivalent of the axial direction. In order to have a better perspective the two dimensional axisymmetric results were mirrored along the axis. Predicted velocity contours using the non-uniform velocity profiles scaled from the NETL burner from Section 3.2 did not yield good results as compared to the PIV measurements (Figure 3.32). The predicted contours did not match the shape of the recirculation area from the experiments; the axial velocity was higher around the recirculation region. Due to the mismatch between the predictions and the experiments it was decided to use a uniform velocity profile at the inlet.

The specified velocity values for the uniform velocity are the following; $U_{\text {axial }}=7.8[\mathrm{~m} / \mathrm{s}]$, $U_{\text {tangential }}=7.8[\mathrm{~m} / \mathrm{s}]$. A reacting flow simulation was performed using the reduced methane-air mechanism ARM9 (Mallampalli, Chen and Fletcher 1998). The simulation was performed on the same mesh that was previously described. Results are shown in Figure 3.33. 
a)

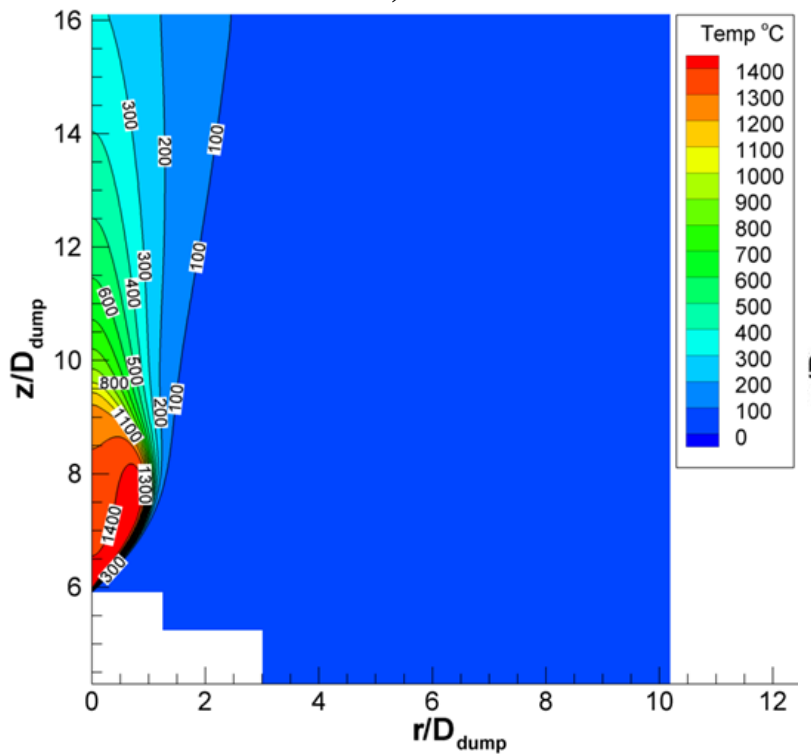

b)

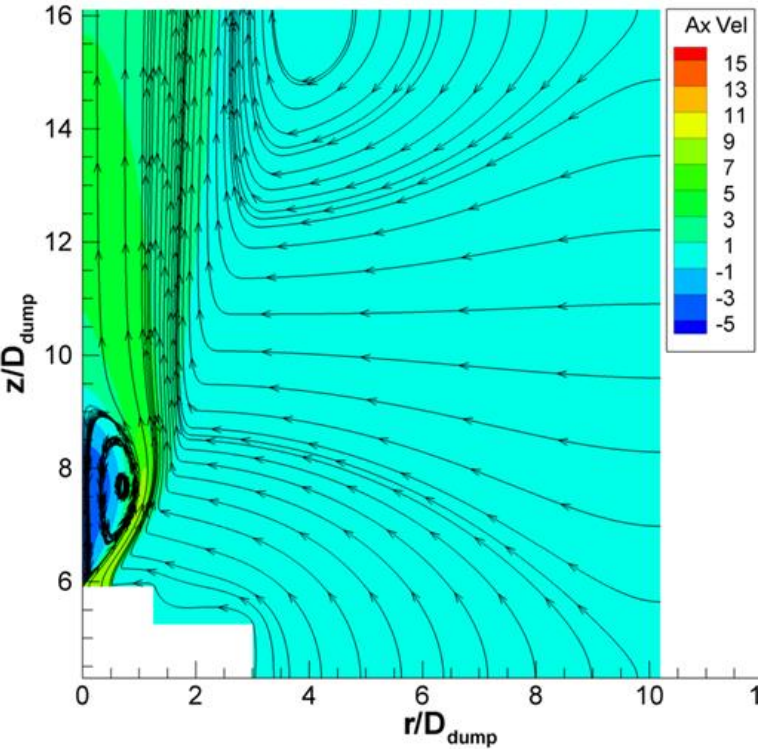

Figure 3.33. Reacting Flow with Uniform Velocity. a) Temperature Contours $\left[{ }^{\circ} \mathrm{C}\right]$, b) Axial Velocity $[\mathrm{m} / \mathrm{s}]$ Contours and Streamlines.

Reacting flow simulations using uniform velocity profiles produced a flame with the expected tulip shape that is characteristic of swirl flames when the central recirculation region is present (Figure 3.33 a)). Figure $3.33 \mathrm{~b}$ ) shows the streamlines and the axial velocity contours. It can be seen how the simulation was able to capture the entrainment of the surrounding fluid in the flame. Pressure outlet boundary conditions specified at the bottom, side and top boundaries allowed the entrainment, but similar simulations using the outflow boundary condition did not capture the entrainment. 
a)

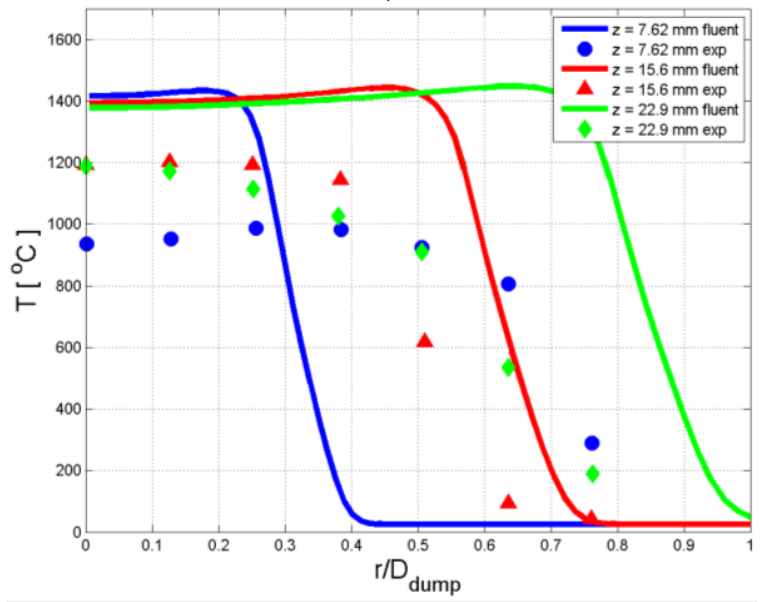

c)

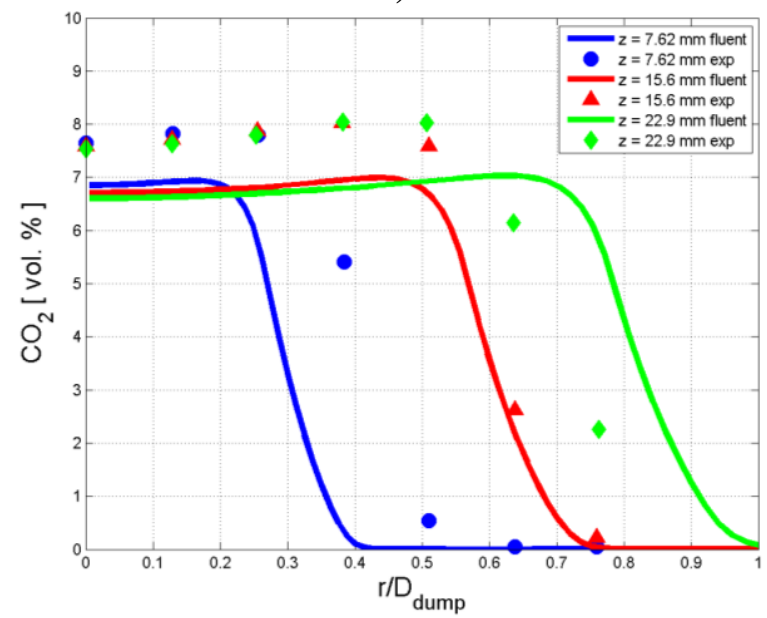

b)

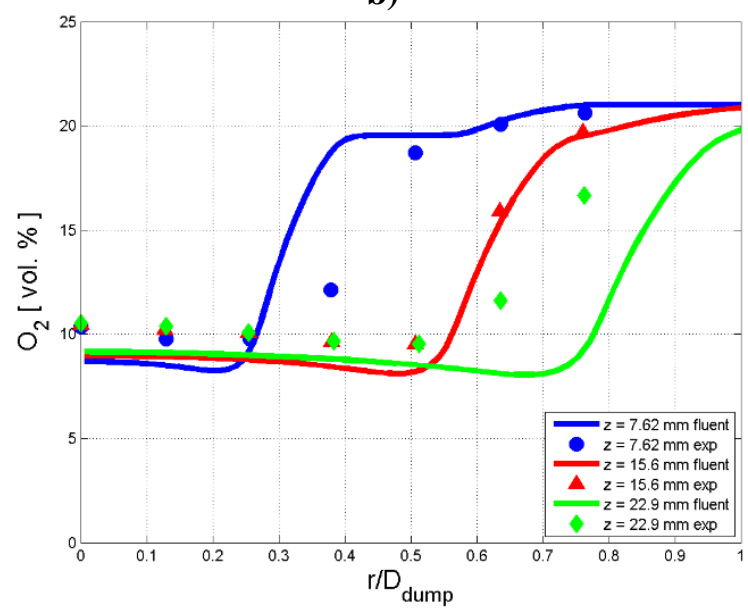

d)

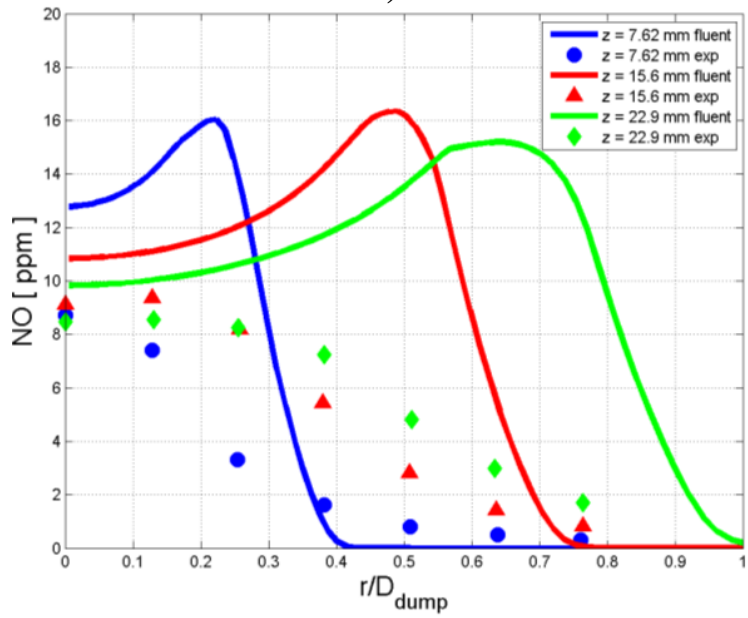

Figure 3.34. Reacting Flow with Uniform Velocity Profiles. a) Temperature Profiles $\left[{ }^{\circ} \mathrm{C}\right]$, b) \% Vol Oxygen, c) \% Vol Carbon Dioxide, d) Nitrogen Oxide [ppm].

Temperature profiles (Figure 3.34 a)) show that the CFD simulation over predicts the flame temperature at the axis $\left(r / D_{\text {dump }}=0\right)$ and the flame area is not predicted correctly. The predicted flame region is narrower near the dump plane (station located at $z=7.62[\mathrm{~mm}]$ ) but further downstream it becomes wider than the experiments (stations located at $z=15.6$, and $z=22.9$ $[\mathrm{mm}])$. Species volume fractions calculated based on a dry analysis are also compared with experiments (Figures 3.34 b) and c)). Volume percentage concentration (volume percentage is equal to the mole fraction multiplied by 100) of oxygen and carbon dioxide captured experimental trend but the predicted profiles are seen to be shifted when compared with experiments (Figures 
$3.34 \mathrm{~b}$ ), and c)). Finally the concentration of NO in parts per million [ppm] is shown in Figure 3.34 d) where the predicted profiles have a maximum value which is not located on the axis of symmetry as compared to the experimental data.

a)

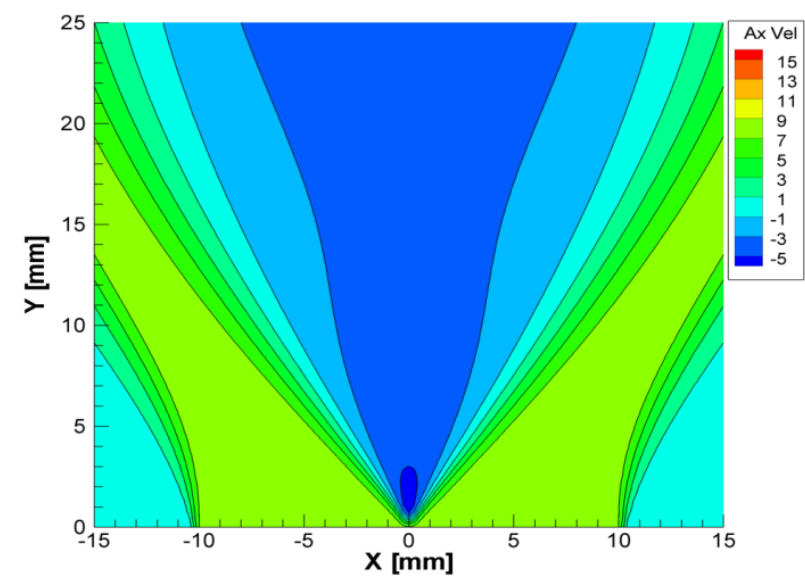

b)

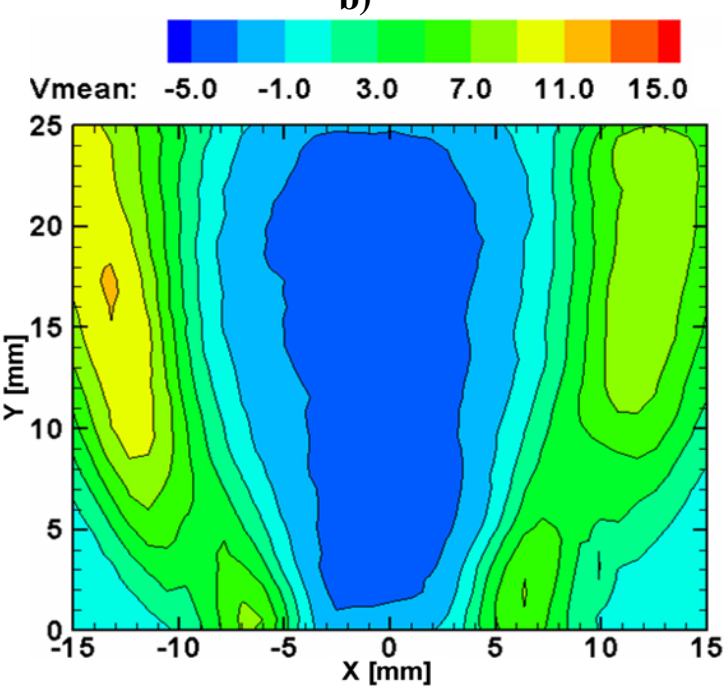

Figure 3.35. Reacting Flow with Uniform Axial Velocity [m/s] Contours. a) CFD Simulations, b) Experiments.

The source of the discrepancy between the predicted temperature and species profiles can be explained by observing the axial velocity contours in Figure 3.35. The central recirculation zone shape was not properly captured by the CFD simulations; the contours showed that the predicted recirculation zone is smaller than what was measured in experiments. Also the shape of the experimental contours shows that the area of negative axial velocity located at $y=0[\mathrm{~mm}]$ extends from $x=-5$ to $5[\mathrm{~mm}]$ in contrast the predicted contours have non negative axial velocity values only at $x=0[\mathrm{~mm}]$.

The results showed the importance of the shape of the inlet velocity profiles in the solution of the temperature and the species concentrations. Chigier and Beer (Chigier and Beer 1964) reported that the swirl number is not the best way to describe swirling flows because it is an integral 
quantity and any properly scaled velocity profiles combination may produce the same swirl number albeit with a significantly different shape of the recirculation zone.

The effect of the inlet velocity profiles is summarized in Figure 3.36.

a)

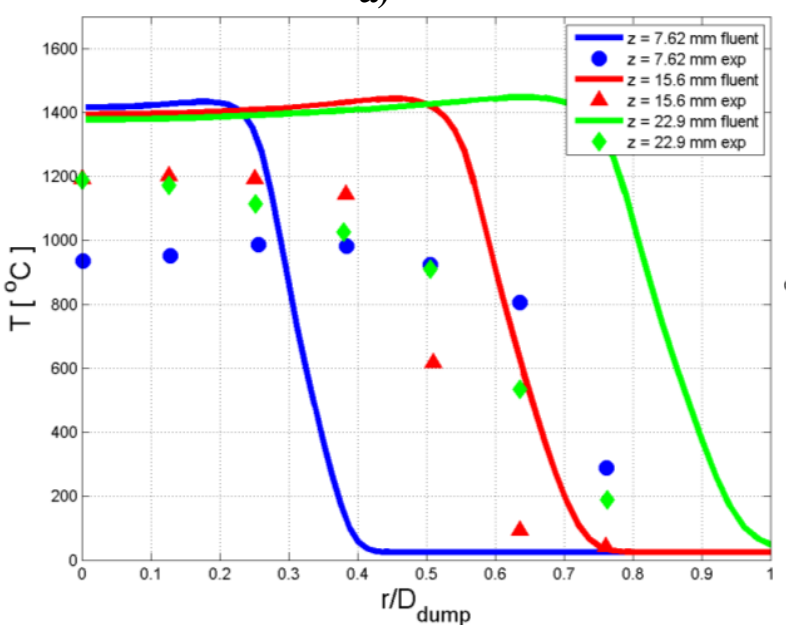

c)

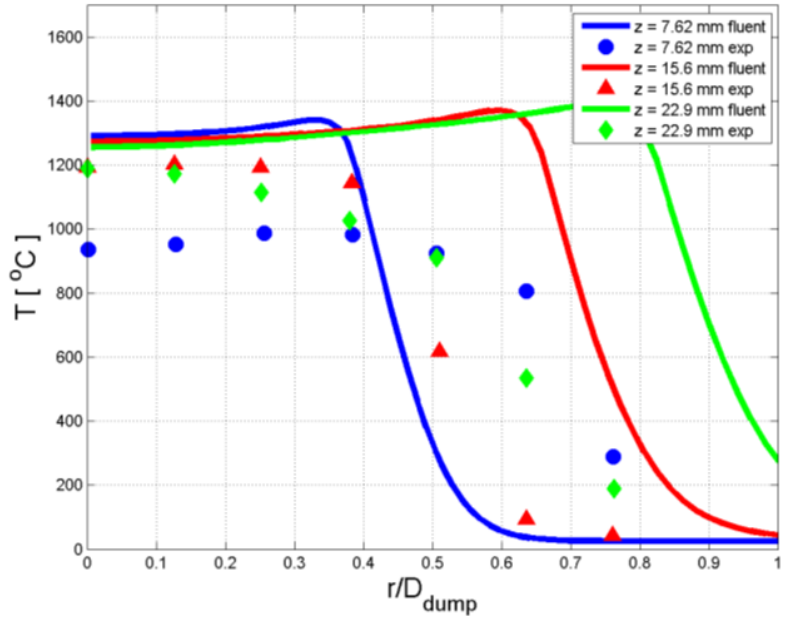

b)

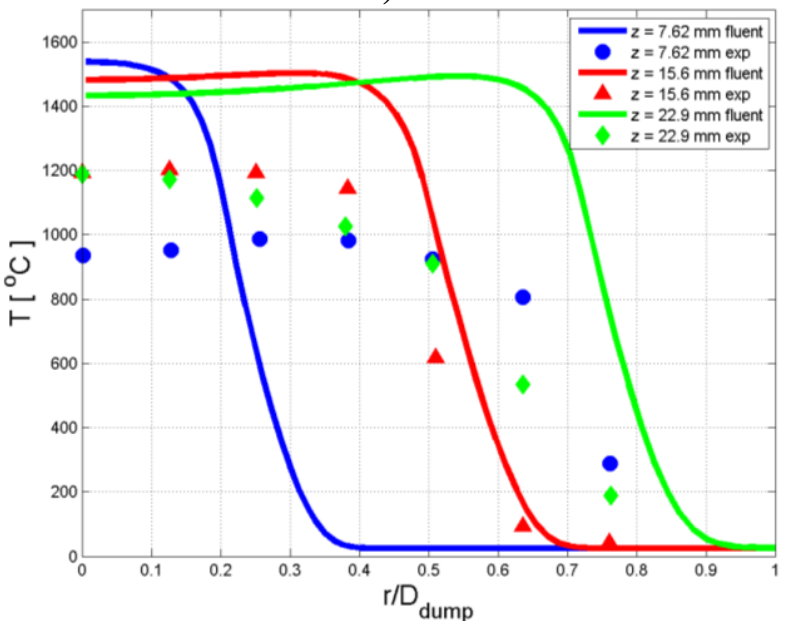

d)

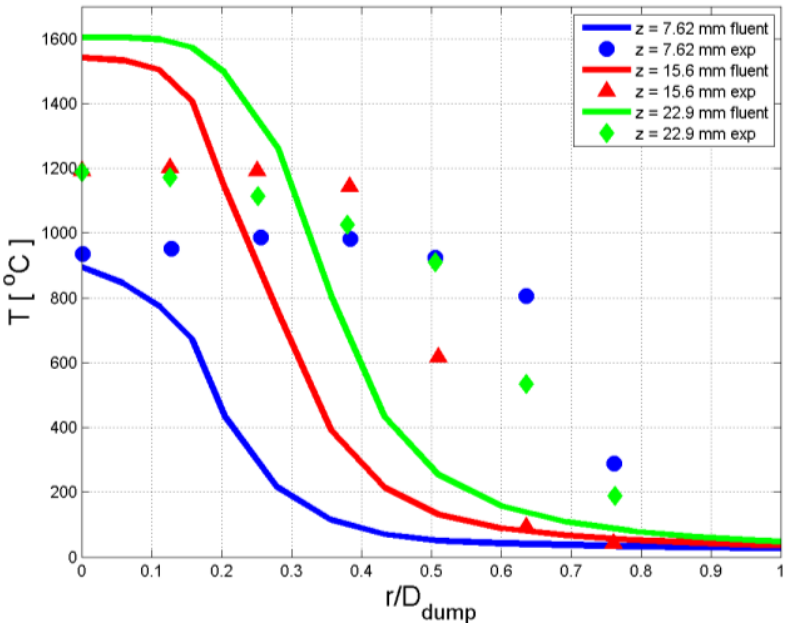


e)

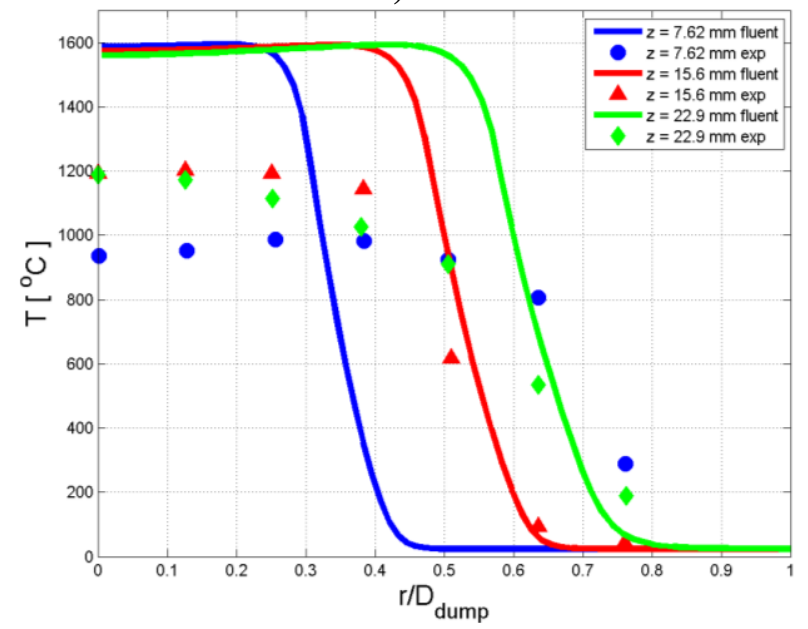

Figure 3.36. Reacting Flow Temperature Profiles. a) Uniform Velocity Profiles, b) Non-Uniform Velocity Profiles Scaled from Chigier and Beer (Chigier and Beer 1964), c) Non-Uniform Velocity from Gupta and Khalil (Gupta and Khalil 2013), d) Three Dimensional Domain using Chigier and Beer Velocity Profiles, e) Three Dimensional Domain with Swirler.

Temperature profiles shown in Figure 3.36 a) were obtained when the inlet profile was uniform as previously discussed.

Figure 3.36 b) shows the predicted temperature profiles that were obtained from a simulation that used the scaled velocity profiles from Chigier and Beer (Chigier and Beer 1964). Chigier and Beer studied a similar configuration as the one reported in the experiments from Kim et al. (Kim, Arghode and Gupta 2009). Here the swirl component of the flow was produced by a coaxial vane swirler and had a convergent region just before the dump plane (similar to the set up in Figure $3.30 \mathrm{~b}$ )). These profiles were scaled in order to match the mass flow and the reported swirl number. The final velocity profiles are shown in Figure $3.31 \mathrm{~b}$ ). Results in Figure $3.36 \mathrm{~b}$ ) showed that the temperature was overpredicted near the axis in a greater amount than the case shown in Figure 3.36 a) and the trend of the temperature remained the same, the flame is narrower at station $y=7.62[\mathrm{~mm}]$ and wider in the subsequent stations.

Experimentally measured velocity profiles provided by Professor Gupta (Gupta and Khalil 2013) were used as inlet velocity profiles in the results shown in Figure 3.36 c). As it can be seen 
from Figure $3.31 \mathrm{c}$ ), the profiles have negative values for the three velocity components near the dump plane. Figure $3.36 \mathrm{c}$ ) shows that temperature over prediction near the axis is the smallest of all the rest of calculations (around $400\left[{ }^{\circ} \mathrm{C}\right]$ ) but the trend of the profiles remained same.

Three dimensional simulations using the domain described in Figure $3.29 \mathrm{~b}$ ) were calculated without including the swirler generator. Non-uniform velocity profiles were prescribed in the inlet area of the domain. Profiles shown in Figure $3.31 \mathrm{~b}$ ) were applied on the inlet surface. The three dimensional computational domain was composed of 114,048 cells but still the grid resolution is coarser than the two dimensional axisymmetric case. Reported results in Figure $3.36 \mathrm{~d}$ ) showed the temperature at station $z=7.62[\mathrm{~mm}]$ closely matches the experimental value but the reported values at the two other stations show that the temperature is overpredicted by an average of $350\left[{ }^{\circ} \mathrm{C}\right]$. The temperature at the first station is significantly lower than what was calculated using the same inlet velocity profiles as in the two dimensional axisymmetric domain (Figure 3.36 b)). The reduction of temperature is due the coarser mesh used in the three dimensional domain. The utilization of coarse meshes in numerical simulations increases the amount of numerical diffusion. The numerical diffusion (or false diffusion) is a source of error in CFD calculations (Versteeg and Malalasekera 2007); the error introduced in the three dimensional calculation made the predicted temperature of the first station lower than the experiments but the calculated profiles of temperature were overpredicted further downstream. The difference between the maximum temperature of the first station with the last station is the largest in the three dimensional coarse mesh case than in the rest of the two dimensional cases.

Figure 3.36 e) shows the temperature profiles obtained using the approach described next. First, a non-reacting flow simulation of the three dimensional domain that includes the inlet swirler and the cone region was performed, Figures $3.28 \mathrm{c}$ ) and $3.29 \mathrm{c})$. The non-reacting flow simulations were calculated using the steady state approach along with the standard k-epsilon model. Profiles 
located at the dump plane were extracted and fitted with a polynomial following the same procedure described in Section 3.2.3. Velocity profiles from non-reacting simulations were imposed at the inlet region of the two dimensional axisymmetric domain described in Figures 3.28 a) and 3.29 a). Predicted temperature profiles showed that the temperature near the domain axis is over predicted by $700\left[{ }^{\circ} \mathrm{C}\right]$ for the first station $(z=7.62[\mathrm{~mm}])$. The temperature profiles showed the same trend as in the other numerical simulations but could not capture what was measured in the experiments.

In summary, the closest prediction to the experiments is obtained in the case shown in Figure $3.36 \mathrm{c})$. This case used the experimentally measured velocity profiles at the inlet. However the trend of the temperature could not be captured, the predicted flame is narrower in the simulations for the first station $(z=7.62[\mathrm{~mm}])$ but wider for the other two stations. Nevertheless Figure $3.36 \mathrm{c}$ ) shows the importance of the shape of the inlet velocity profiles. It is seen that even with the axial velocity profile that match the mass flow rate and the tangential velocity profile that produced the desired swirl number, the shape of the recirculation area, the temperature field, nor the species concentrations, could not be captured as measured in experiments.

a)

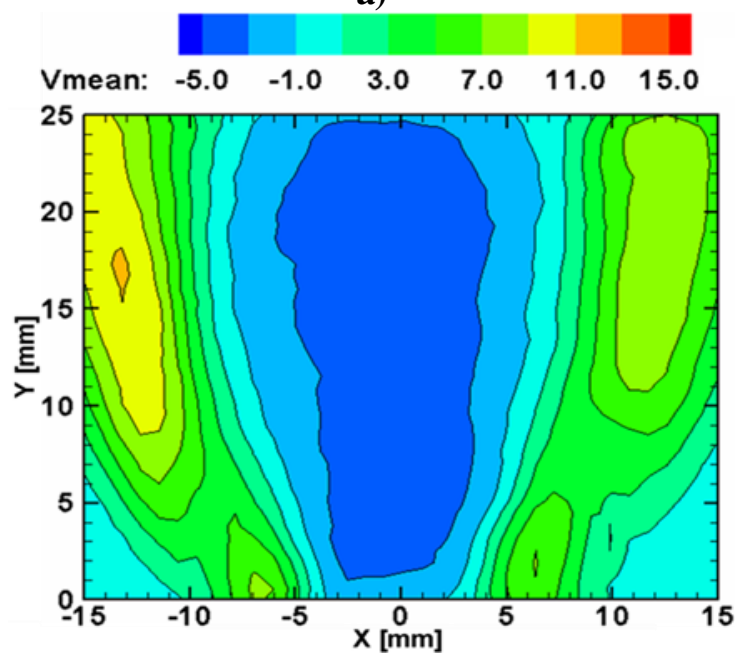

b)

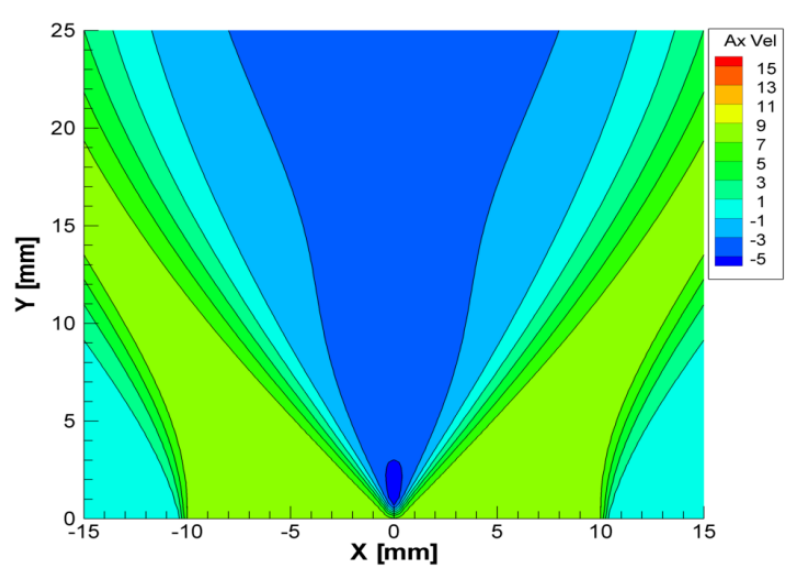


c)

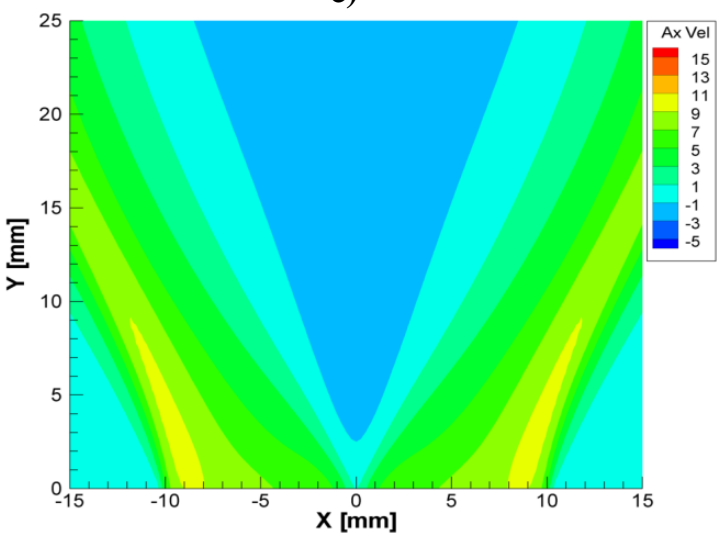

e)

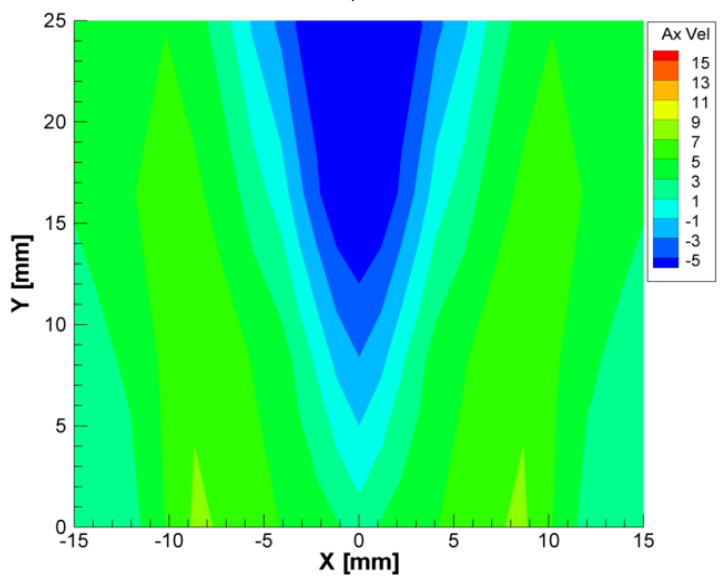

d)

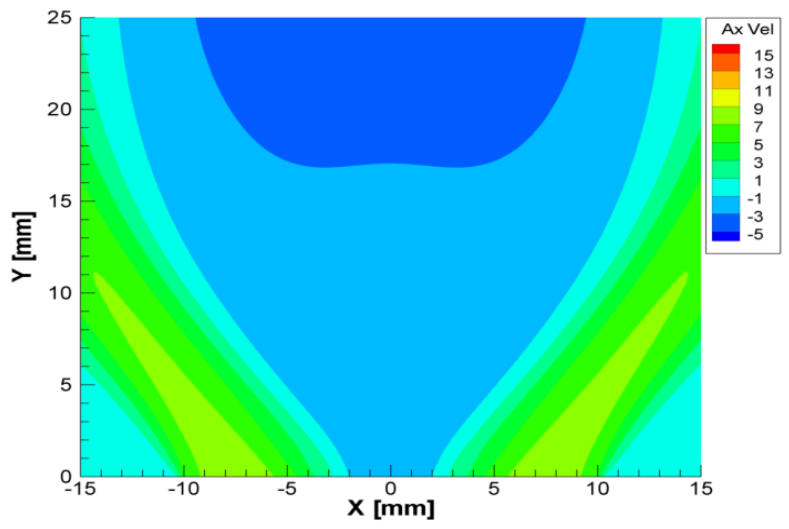

f)

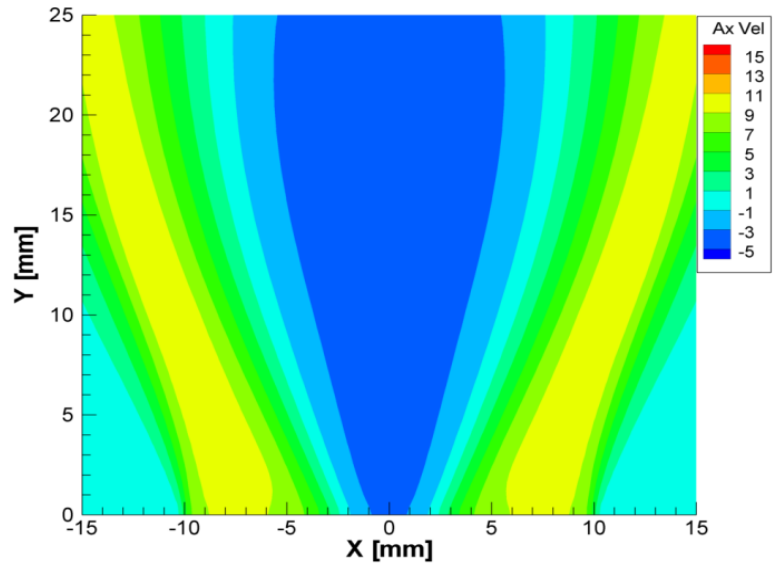

Figure 3.37. Reacting Flow Axial Velocity [m/s] Contours. a) Experiments, b) Uniform Velocity Profiles, c) Non-Uniform Velocity Profiles Scaled from Chigier and Beer (Chigier and Beer 1964), d) Non-Uniform Velocity from Gupta and Khalil (Gupta and Khalil 2013), e) Three Dimensional Domain using Chigier and Beer Velocity Profiles, f) Three Dimensional Domain with Swirler.

It can be seen from the axial velocity contours shown in Figure 3.37 d) that negative axial velocity contour at $y=0[\mathrm{~mm}]$ extends the most of all the predicted contours but not as much as in the experiments. The recirculation zone in $3.37 \mathrm{~d}$ ) is wider compared to experiments in the downstream direction. It was not possible to predict the flame with enough accuracy in the aforementioned simulations. Several more simulations were performed without significant improvement in the temperature and velocity predictions. The additional simulations included the utilization of different turbulence models (e.g. RSM, k-omega SST) and different higher order discretization schemes of the convective terms (e.g. QUICK, MUSCL). Grid refinement and coarsening was also performed but no significant improvement was achieved. 
The only approach that was not used for the simulation was the Large Eddy Simulations (LES), this method was not employed because of the excessive amount of time needed to calculate the solution (around weeks) which stands against the main objective of the present study which is to reduce the calculation time of combustion phenomena. Due to the fact that the numerical results did not match the trend of the measured temperature profiles these results were not used in the chemical reactor network calculations. The application of the CRN approach for the previous cases are presented in the Chapter 4. 


\section{Chapter 4: $\quad$ Chemical Reactor Network Simulations}

The main goal of the chemical reactor network $(\mathrm{CRN})$ is to simplify the domain of the combustor in zones that share similar conditions, these zones are called reactors. Current CFD solution approach consists in the simultaneous solution of momentum, energy, turbulence, and species transport equations. As explained in the Introduction chapter the main bottle neck in combustion simulations is the chemistry modelling. Even for methane which is one of the most commonly used fuels, CFD solution becomes impractical to simulate using detailed chemistry with current computational resources. The GRI 3.0 detailed mechanism (Smith, et al. 1999) is composed of 325 reactions and 53 chemical species. This means that 53 partial differential equations (one scalar transport equation for each of the chemical species) must be added to the problem in order to simulate detailed chemistry. Adding detailed chemistry to CFD simulations is limited to simple fuels (e.g. hydrogen) and to very simple problem geometries and low Reynolds numbers. In order to reduce the problem complexity, reduced chemistry mechanisms have been developed. These models start with the assumption that a limited number of chemical species play an important role in the problem. This assumption is partially true and depends on what the goal of the simulation is. For example, two step global mechanism for methane that includes six chemical species $\left(\mathrm{CH}_{4}, \mathrm{O}_{2}\right.$, $\mathrm{H}_{2} \mathrm{O}, \mathrm{CO}_{2}, \mathrm{CO}, \mathrm{N}_{2}$ ) can satisfactory predict the heat release of the system, but if the goal is to predict the pollutant emissions, this mechanism cannot be used because it does not include NO specie. A popular NO mechanism for methane-air flames is the ARM9 (Mallampalli, Chen and Fletcher 1998) (ARM stands for Augmented Reduced Mechanism) which contains nine chemical species $\left(\mathrm{CH}_{4}, \mathrm{O}_{2}, \mathrm{H}_{2} \mathrm{O}, \mathrm{CO}_{2}, \mathrm{CO}, \mathrm{N}_{2}, \mathrm{H}_{2}, \mathrm{OH}, \mathrm{NO}\right)$. The ARM9 is a reduced version of the GRI 2.1 detailed mechanism which was modified assuming that the rest of the chemical species are in equilibrium or quasi steady state. A sensitivity calculation was performed in order to assess that the chosen chemical species do predict the system heat release and products composition. CPU time needed to perform CFD simulations using the ARM9 mechanism increases considerably compared 
to that of the global step mechanisms, mostly due to the integration of the chemical source terms. However other CFD applications may require a better prediction of the concentration of NO. For the cases in which the prompt NO pathway formation is dominant (usually at low fuel-air equivalence ratio) the ARM9 may not give accurate predictions because it was optimized to predict the thermal NO but not the NO production via the prompt pathway; the HCN chemical specie is critical for the calculation of prompt NO but the ARM9 assumes it to be in equilibrium (Turns 1996) leading to incorrect predictions (Escobar, et al. 2010). In order to tackle the aforementioned problem Sung et al. (Sung, Law and Chen 2001) developed the ARM19 reduced mechanism. This mechanism contains 19 chemical species $\left(\mathrm{CH}_{4}, \mathrm{O}_{2}, \mathrm{H}_{2} \mathrm{O}, \mathrm{CO}_{2}, \mathrm{CO}, \mathrm{N}_{2}, \mathrm{H}_{2}, \mathrm{OH}, \mathrm{NO}, \mathrm{H}, \mathrm{HO}_{2}, \mathrm{H}_{2} \mathrm{O}_{2}\right.$, $\left.\mathrm{CH}_{3}, \mathrm{CH}_{2} \mathrm{O}, \mathrm{C}_{2} \mathrm{H}_{2}, \mathrm{C}_{2} \mathrm{H}_{4}, \mathrm{C}_{2} \mathrm{H}_{6}, \mathrm{HCN}, \mathrm{NH}_{3}\right) \mathrm{HCN}$ being a part of the solution. ARM19 mechanism performs better for prompt NO calculation but the computational time increases dramatically. A simple calculation using ARM9 may take only one day, on the other hand a solution using ARM19 may take up to one week. Moreover the complexity that is added by the extra species and assumptions involved in the ARM19 mechanism makes it extremely difficult to achieve a converged solution.

Due to excessive computational time needed for CFD solutions that employ detailed chemical mechanisms, the $\mathrm{CRN}$ was introduced to reduce the computational time. The CRN approach is based on the simplification of the physical domain using ideal reactor models. For example the computational domain can be divided into zones that share the same temperature and composition in terms of some selected species. The zones are also called reactors and can be simulated as an ideal zero dimensional domain, or one dimensional domain. In order to perform the zone division, it is necessary to know how the flow, temperature and species concentration vary inside the combustor. The characterization of the combustor can be performed using the results from CFD simulations. Temperature, flow and composition contours can be obtained from CFD results. In order to accelerate the simulations the present study used relatively simple computational 
models such as two dimensional axisymmetric geometries, RANS to model the turbulent flow, and the implementation of simple chemical mechanisms to characterize the burner. The information obtained from the CFD model was used as input in a MATLAB program designed to cluster the computational cells into regions that share similar values of the control variables. Control variables are chosen by the user and usually correspond to the temperature and the concentrations of a selected chemical species. The volume of each region was calculated by adding the volume of every computational cell included in the reactor. An average temperature and concentration value was calculated for each reactor using the information from the CFD results. The connectivity of the reactor zones was given by the sign of the mass flux. Then the inlet boundary conditions are applied and the results are calculated using an iterative procedure. A detailed description of the process is given in the following sections.

\subsection{Zone Selection}

In order to create a network it is necessary to read the results from CFD simulations and cluster the computational cells into regions. The computer cell clustering was achieved using the CFD mesh information. The information needed from the computational mesh is; shape of the computational cells (triangles, rectangles, etc.), position of the nodes, and the connectivity between elements (lines). This information was obtained from the mesh files. Mesh files were imported into MATLAB and reconstruction of the mesh was achieved using an in-house computer program.

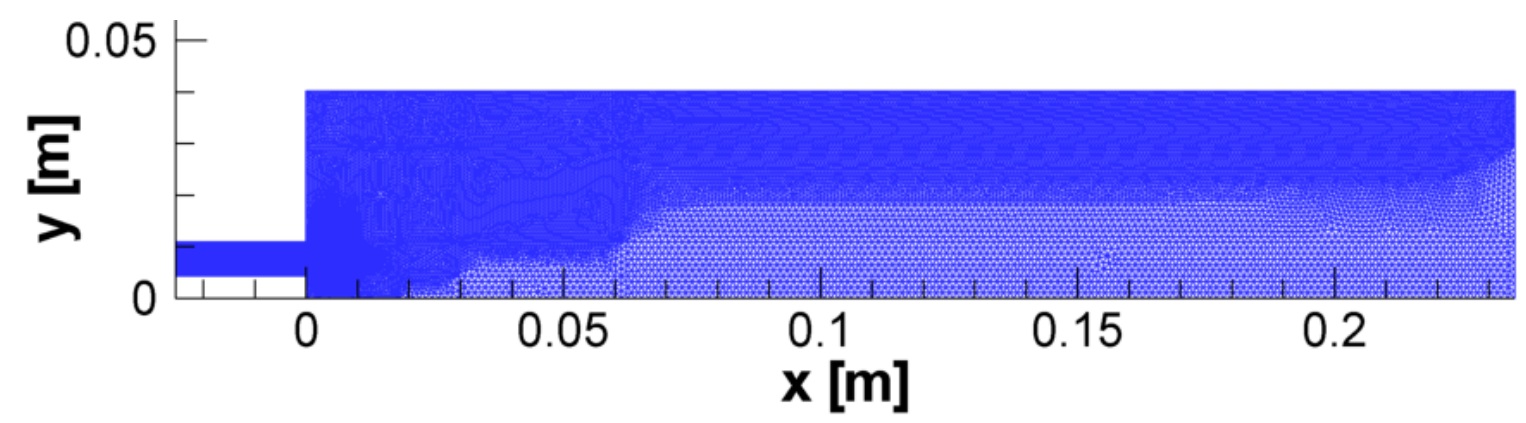

Figure 4.1. Mesh Reconstruction using MATLAB. 
Figure 4.1 shows the mesh reconstruction of the CFD domain using MATLAB, which will be referred to CRN-DIV from now on. Once the domain was reconstructed then the information from CFD results can be loaded. Temperature, velocity, composition, and mass flux between cells were written in files that were loaded in the CRN-DIV program. The values were then attached to the corresponding computational cell. At least two control variables can be defined by the user to build the CRN. The levels were defined by equally spaced ranges using

$\Delta \operatorname{Var} r_{1}=\frac{\operatorname{Var}_{1, \max }-\operatorname{Var}_{1, \min }}{n_{\text {var } 1}}$

Where $\operatorname{Var}_{1}$ is the control variable \#1, $n_{\text {varl }}$ is the number of levels defined by the user. The subscript max corresponds to the maximum value of the variable \#1 in the entire computational domain, and $\min$ corresponds to the minimum value, finally $\Delta \operatorname{Var}_{1}$ is the calculated relative difference. Then an array of length $n_{v a r l}$ is created for variable \#1. The same procedure is applied for the rest of the control variables.

In order to create a CRN that represents the combustor behavior it was necessary to properly select the control variables. Temperature was selected as one of the control variables due to the dependence of the chemical reaction to it. Because the utilization of the temperature variable is not enough to properly divide the combustor into several regions, for example it is possible to find similar temperature values near the flame and in the post flame region but these are two different zones because the chemical reactions are more intense near the flame than in the post flame region. Due this it was necessary to select a second control variable. In the present study it was decided to use the mass fraction of one of the combustion products, the selection of the chemical specie depended on the species included in the chemical mechanism used in the CFD simulation. It was necessary to select a chemical specie that clearly divided the reactant inlet section, the flame region, and the post flame region. In the present study carbon dioxide was 
selected for the NETL burner case but water mass fraction was selected for the TECFLAM burner case. Water was selected for the TECFLAM burner because this configuration had walls that were maintained at relatively high temperature $\left(T_{\text {wall }}=1073[\mathrm{~K}]\right)$, this temperature allowed the dissociation of carbon dioxide into carbon monoxide making the correct division of the burner domain more difficult. On the other hand, water mass fraction stayed almost constant in the post flame region.

The zone separation is performed by looping through all the computational cells in the domain and identifying the cells that fall in the levels of the control variables previously defined by the user as follows:

$$
\begin{aligned}
& \text { Looping from } k=1 \text { to } n_{\text {cells, CFD }} \\
& \text { Looping from } i=1 \text { to } n_{\text {varl }}-1 \\
& \text { If } \operatorname{Var}_{1}(i)<\operatorname{Var}_{C F D}(k)<=\operatorname{Var}_{1}(i+1) \\
& \operatorname{Looping}_{\operatorname{from} j=1 \text { to }} n_{\text {var } 2} \\
& \text { If } \operatorname{Var}_{2}(j)<\operatorname{Var}_{C F D}(k)<=\operatorname{Var}_{2}(j+1) \\
& \text { id_cell } \\
& \text { Var,CFD } \\
& \text { (k) }=(i-1) * n_{\text {Varl }}+j
\end{aligned}
$$

Equation 4.2 shows that if the value of the CFD variable falls in one of the intersections of the two control variables a value of id_cell variable is assigned to the cell. This process is performed for all the computational cells loaded from the CFD simulation. Cells with identical id_cell values are clustered in the same reactor. An example of the cell clustering is shown in Figure 4.2. 
a)

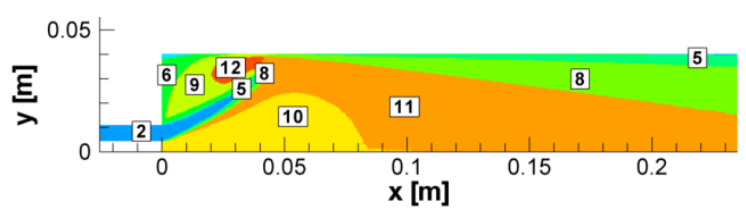

b)

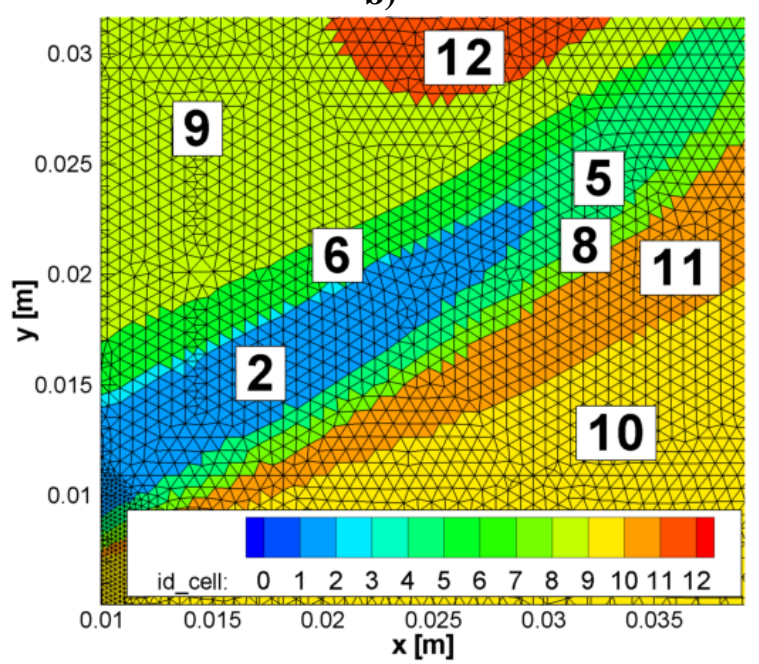

Figure 4.2. Zone Division of the CFD Domain. a) Complete Domain, b) Flame Region.

Each color in Figure 4.2 corresponds to a different zone or reactor. For each reactor the total volume is calculated by adding the volume of each cell contained in the zone. Reactor averaged value of temperature and composition are calculated using a volume weighted formulation using Equation 4.3.

$\phi_{\text {avg }}=\frac{\sum_{k=1}^{N_{\text {react }}} \operatorname{Vol}_{k} \phi_{k}}{\text { Vol }_{\text {react }}}$

Where $N_{\text {react }}$ is the total number of computational cells in one reactor, $\operatorname{Vol}_{k}$ is the volume of the $k$ computational cell, $\phi_{k}$ is the variable of interest in the $k$ cell, and $V_{o l} l_{\text {react }}$ is the total volume of the reactor.

Once the reactors are identified the next step is to loop over all the computational cells and check if the neighbor cells have the same id_cell value. If two neighbor cells have a different id_cell value they are marked as boundaries. In order to perform this operation it is necessary to know the connectivity between the computational cells (i.e. the neighbors of each computational cell); this information is contained in the previously loaded mesh files. 


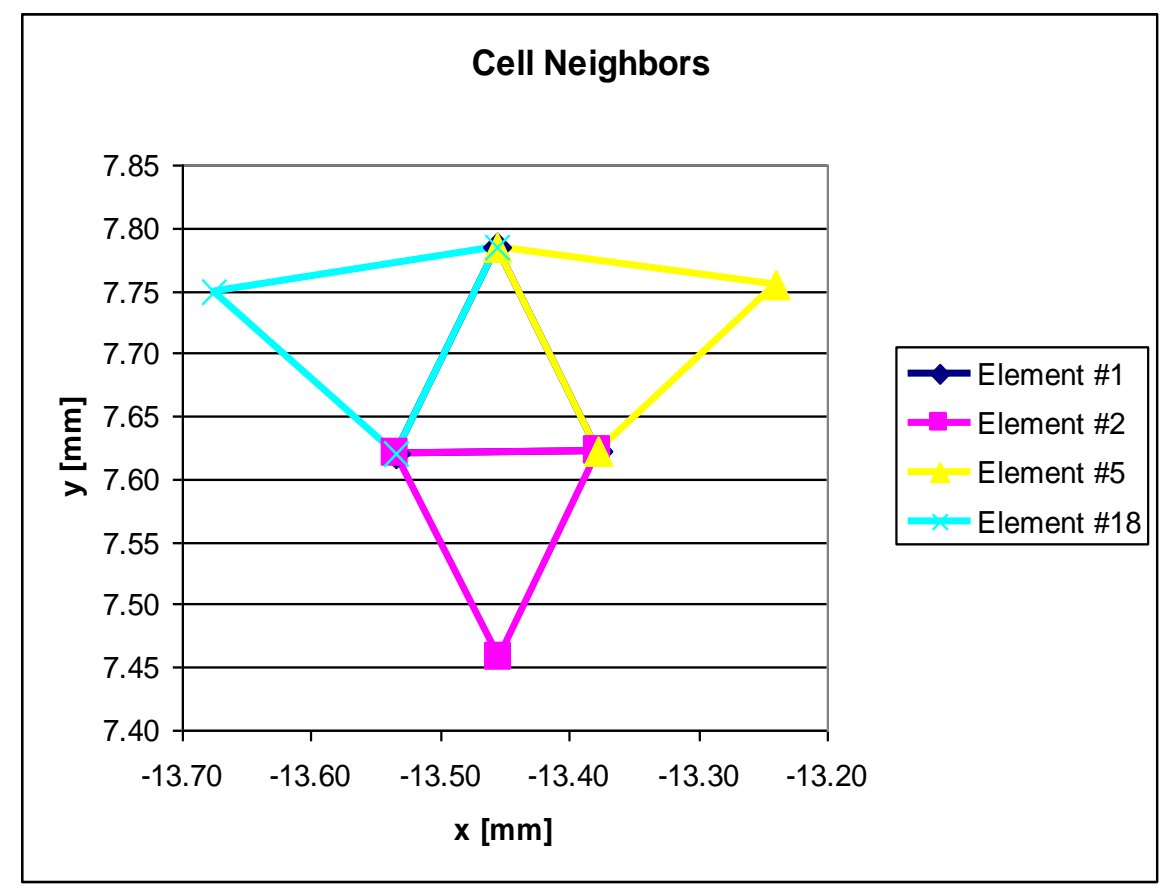

Figure 4.3. Cell Neighbors.

Figure 4.3 shows an example of the boundary marker process. The process starts by setting the pivot cell, Element \#1, then the CRN-DIV program searches in the loaded mesh data for the neighboring cells and retrieves the results, Elements \#2, \#5, and \#18. CRN-DIV checks the id_cell value of the pivot cell and its neighbors if one of the neighbors shown in Figure 4.3 does not have the same id_cell value as the pivot cell then the two cells are marked as being a boundary. As an example marked boundaries are shown in Figure 4.4 (red elements). 
a)

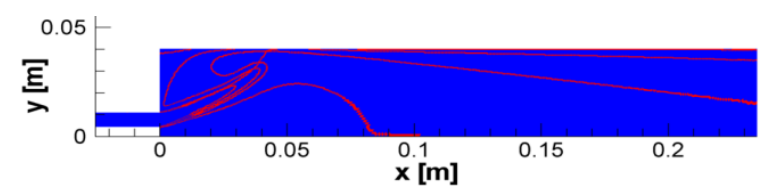

Figure 4.4. Zone Boundaries. a) Complete Domain, b) Flame Region. b)

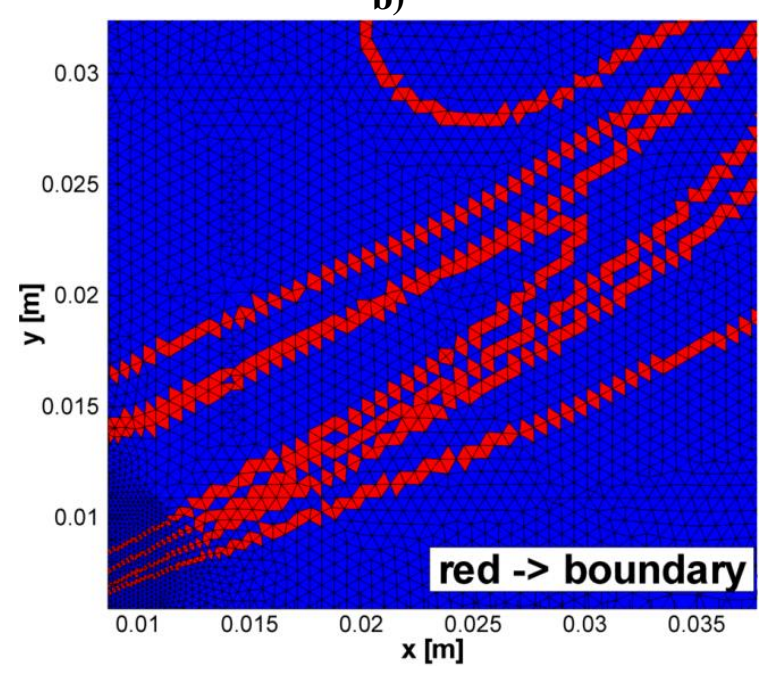

Mass flux between boundaries can be calculated with the boundary information. Once the boundaries were identified then the mass flux through the cell face is calculated using a built-in function in ANSYS-FLUENT called F_FLUX. As an alternative Equation 4.4 was also considered.

$\dot{m}_{\text {convective }}=\rho_{\text {face }} \vec{V}_{\text {face }} \cdot d \vec{A}_{\text {face }}$

It was found that the F_FLUX produces a better mass balance than results obtained using Equation 4.4. F_FLUX calculates the mass flux with more accuracy because ANSYS-FLUENT saves the variables at the cell center. When Equation 4.4 was used the velocity was interpolated from the cell center to the cell face using a harmonic average but this interpolation proved to be problematic because the velocity used in FLUENT and in any finite volume solver depends on the discretization scheme used. In order to calculate the correct face velocity it is necessary to interpolate the velocity from the cell centers using the convective discretization scheme. For the current calculations a $2^{\text {nd }}$ order upwind scheme is used. This process turned out to be not trivial because of the non-uniform computational mesh used in the simulations. On the other hand the F_FLUX function takes into account the aforementioned details giving a better calculation of the 
cell mass flux. Another important detail found was that the best results of the cell flux were achieved when the simulation not only converged to the desired scaled residuals (in this case lower than $10^{-5}$ ) but the values monitored at selected critical points did not change significatively (less than $1 \%$ change with respect the value of the same quantity from the previous iteration). Temperature, velocity, and mass fraction of at least one species at selected points were closely monitored. Once the residuals were lower than the desired value and the monitors were flat, the solution was considered converged. F_FLUX calculations gave better results when the two conditions were met and not only that the residuals were lower than the desired value.

The total mass flux between two neighbor reactors was calculated from

$\dot{m}_{i-j}=\sum_{k=1}^{N_{\text {boundary }, i-j}} \dot{m}_{i-j, k}$

Where $\dot{m}_{i-j}$ is the mass flux between reactors $i$ and $j, N_{\text {boundary, } i-j}$ is the total number of computational cells in the boundary between the reactors $i$ and $j$, and $\dot{m}_{i-j, k}$ is the single cell mass flux between reactors $i$ and $j$.

The CRN-DIV program produces two data files, one contains the total number of reactors with their respective volumes, and averaged quantities of temperature and composition. The second data file has the mass flux information between reactors along with the direction of the mass transfer. With the information from the second data file it is possible to know how the reactors connect and the direction of the mass flux. An example CRN is shown in Figure 4.5. 


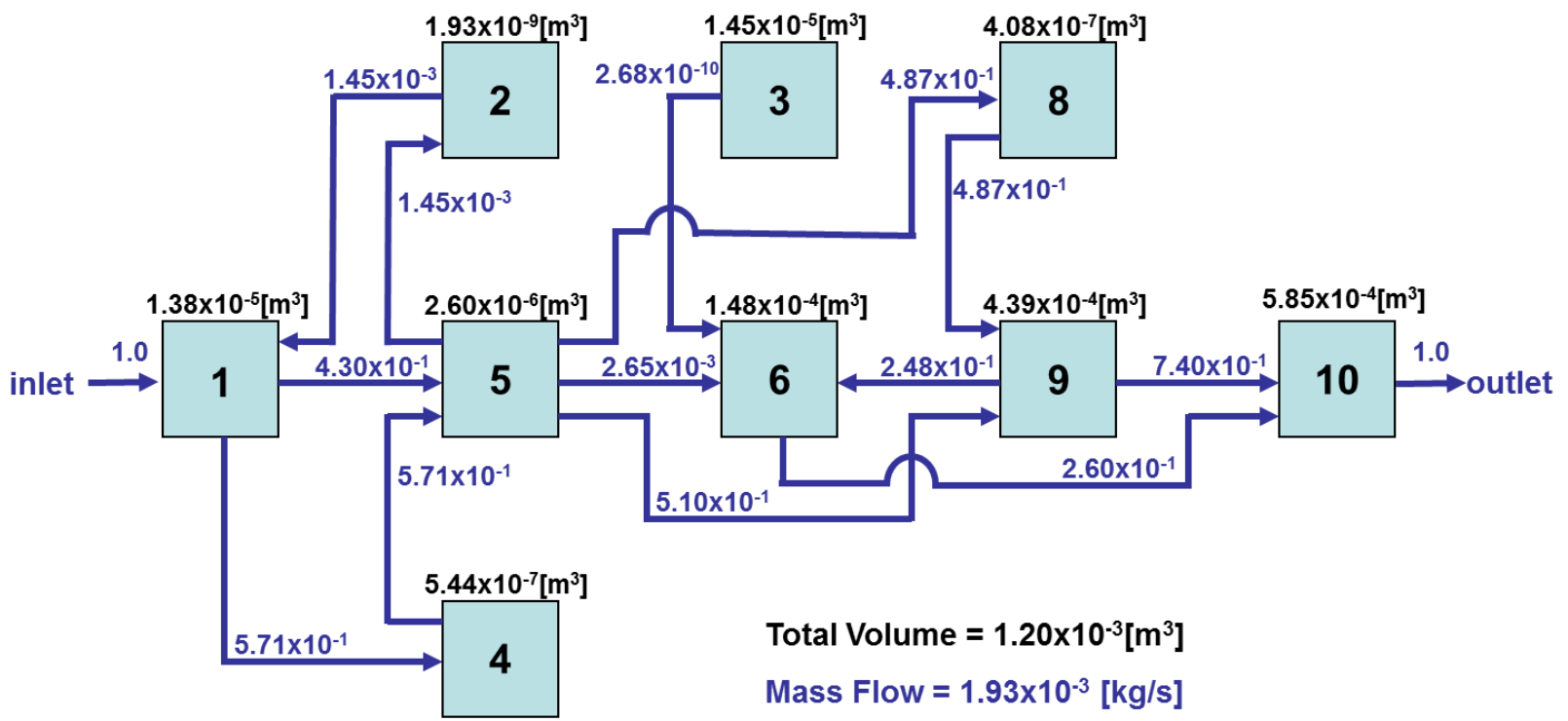

Figure 4.5. Example of a CRN Layout.

Figure 4.5 shows a CRN layout that corresponds to the NETL-Burner. The details of the zone division for the NETL burner are discussed in sections 4.3.1 and 4.3.2 of the present study. Each square in Figure 4.5 represents a simplified reactor and its volume is given at the top of each element, the summation of the volumes of the reactors was equal to the total volume of the combustor. The connection of the network was given by the direction of the mass flux. Figure 4.5 shows the values of the fluxes which were normalized with the total mass flux that enters into the combustor. It can be seen that the mass was conserved for the CRN but for reactor 3, here there was only mass going out however this value was extremely small $\left(\sim 10^{-10}\right)$ and did not significantly affect the total mass balance (mass imbalance $\sim 10^{-8} \%$ ). What is not given by the CRN-DIV code is the inlet composition and temperature. This information is prescribed separately and will be discussed in the next section. In some cases the domain outlet is composed of several reactors. In this situation the outlet compositions were calculated as a weighted area average of the zones located at the outlet using Equation 4.6. 


$$
X_{j, \text { outlet }}=\sum_{\text {reactor }}^{N_{\text {oultet }}} \frac{\text { area }_{\text {reactor }}}{\text { area }_{\text {outlet, } \text {,ombustor }}} * X_{j, \text { reactor }}
$$

Where $X_{j}$ is the mole fraction of the specie $j, N_{\text {outlet }}$ is the total number of reactors located at the domain outlet, area $_{\text {reactor }}$ is the area of the outlet covered by a zone reactor, and area $_{\text {outlet,combustor }}$ is the total area outlet of the combustor domain.

\subsection{Chemical Reactor Network Code}

In order to simulate the CRN a computer program was developed in FORTRAN. The code simulates each reactor of the network using a Perfectly Stirred Reactor (PSR) or a Plug Flow Reactor (PFR), each reactor will be briefly explained later in this chapter. The flow diagram of the CRN program is in Figure 4.6.

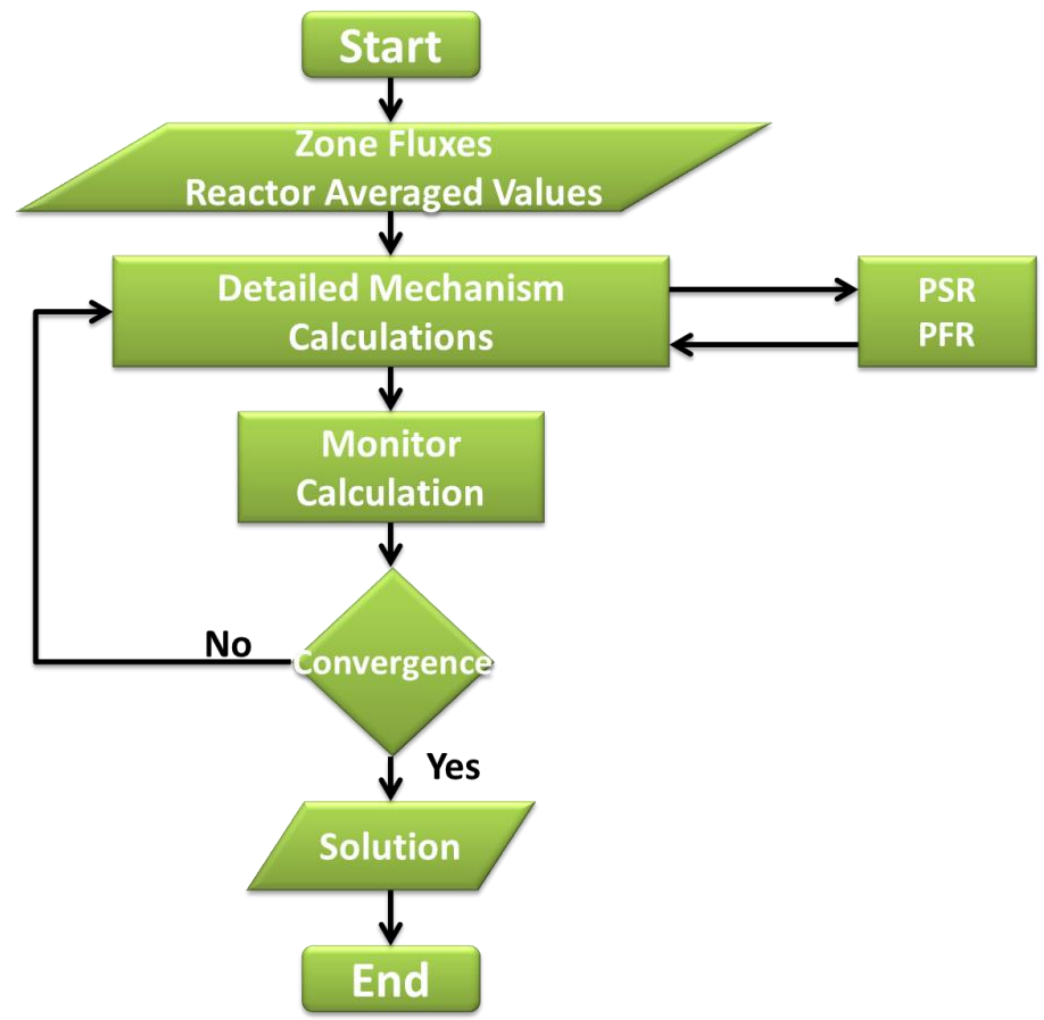

Figure 4.6. Flow Diagram of the CRN Computer Program. 
The CRN program reads the output data created in the CRN-DIV program. The inlet composition that enters to each one of the reactors is calculated as a weighted average from

$X_{j, \text { pivot_in }}=\sum_{k=1}^{N_{\max , i n}} \frac{\dot{n}_{k}}{\dot{n}_{t o t}} X_{j, k}$

Where $N_{\max , \text { in }}$ is the maximum number of reactors that deliver mass to the pivot reactor, $\dot{n}_{k}$ is the mole flow rate delivered by the $k$ neighbor reactor, $\dot{n}_{t o t}$ is the total molar flow rate that enters into the pivot reactor, $X_{j, k}$ is the mole fraction of the specie $j$ in the $k$ reactor.

The inlet composition and temperature were set equal to the boundary conditions of the problem. Each reactor is simulated at constant temperature, the temperature was set equal to the average temperature. The reactor composition was sent as input along with the temperature and the volume to the external programs that solved the PSR or PFR. The GRI 3.0 detailed mechanism was used in the solution of the PSR or PFR. The predicted concentrations were used to calculate the inlet composition using Equation 4.7. The present simulations used the mole fraction of NO, water, and carbon monoxide as monitors. The solution procedure of the CRN is an iterative process that was repeated until convergence was achieved.

Simulation of the CRN was performed at constant temperature because one of the main assumptions of the CRN is that the extra species included in the detailed mechanism are "minor" species and they do not affect significatively the temperature or the velocity field obtained from the CFD model.

\subsubsection{Perfectly Stirred Reactor (PSR)}

The perfectly stirred reactor (PSR) model assumes that the species are so well mixed that there are no spatial gradients inside the reactor. This means that the temperature and composition 
are the same at any location inside the reactor. For this reason this reactor can be treated as a zero dimensional model. A diagram of the PSR is shown in Figure 4.7

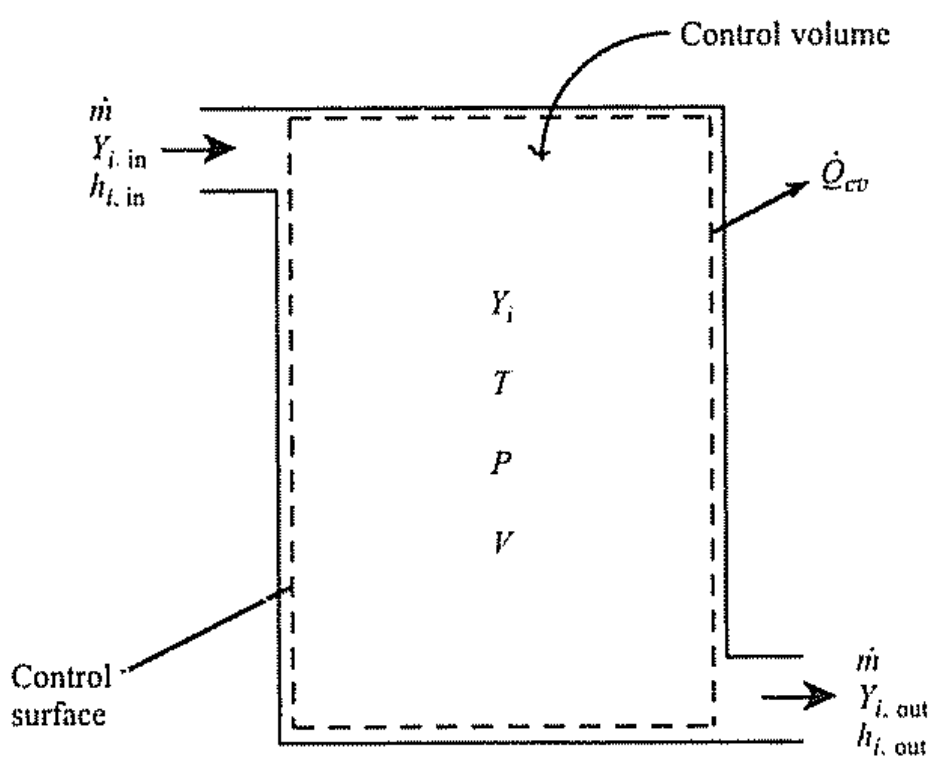

Figure 4.7. Perfectly Stirred Reactor (PSR) concept.

The relevant input parameters for the PSR are: the inlet mass flow rate $\dot{m}$, the inlet composition $Y_{\text {in }}$, the reactor temperature $T$, and the reactor volume $V$.

The steady state specie transport equation of the PSR is given by

$\dot{\omega}_{j} M W_{j} V+\dot{m}\left(Y_{j, \text { in }}-Y_{j, \text { out }}\right)=0$

Where $\dot{\omega}_{j}$ is the net production rate of specie $j$, and $M W_{j}$ is the molecular weight of specie $j$.

The mass inlet flow rate, $\dot{m}$, of the reactor was set equal to the calculated value from the CRN-DIV program. Being at constant temperature without heat loss it is not necessary to solve the energy equation for a PSR. Solution of Equation 4.8 is performed using the modified Newton method which is explained in detail in Glarborg, et al. (1986) and Turns (1996). 


\subsubsection{Plug Flow Reactor (PFR)}

It is necessary to include the PFR model in the combustor simulation especially in the postflame region where the flow is usually one dimensional and the gradients of temperature are mainly one dimensional as well. In order to simulate this component of the network it was decided to use the approach given in Levenspiel (Levenspiel 1962) which consists of the utilization of a series of PSRs. This was accomplished by the discretization of the PFR domain into several regions, each one simulated by one PSR. The simulation of the PFR was accomplished through the development of a driver code written in FORTRAN. The driver inputs the inlet conditions in the PSR code and then runs the calculations followed by the collection of the results which are used as inlet conditions for the next PSR. The flowchart of the driver PFR code is depicted in Figure 4.8.

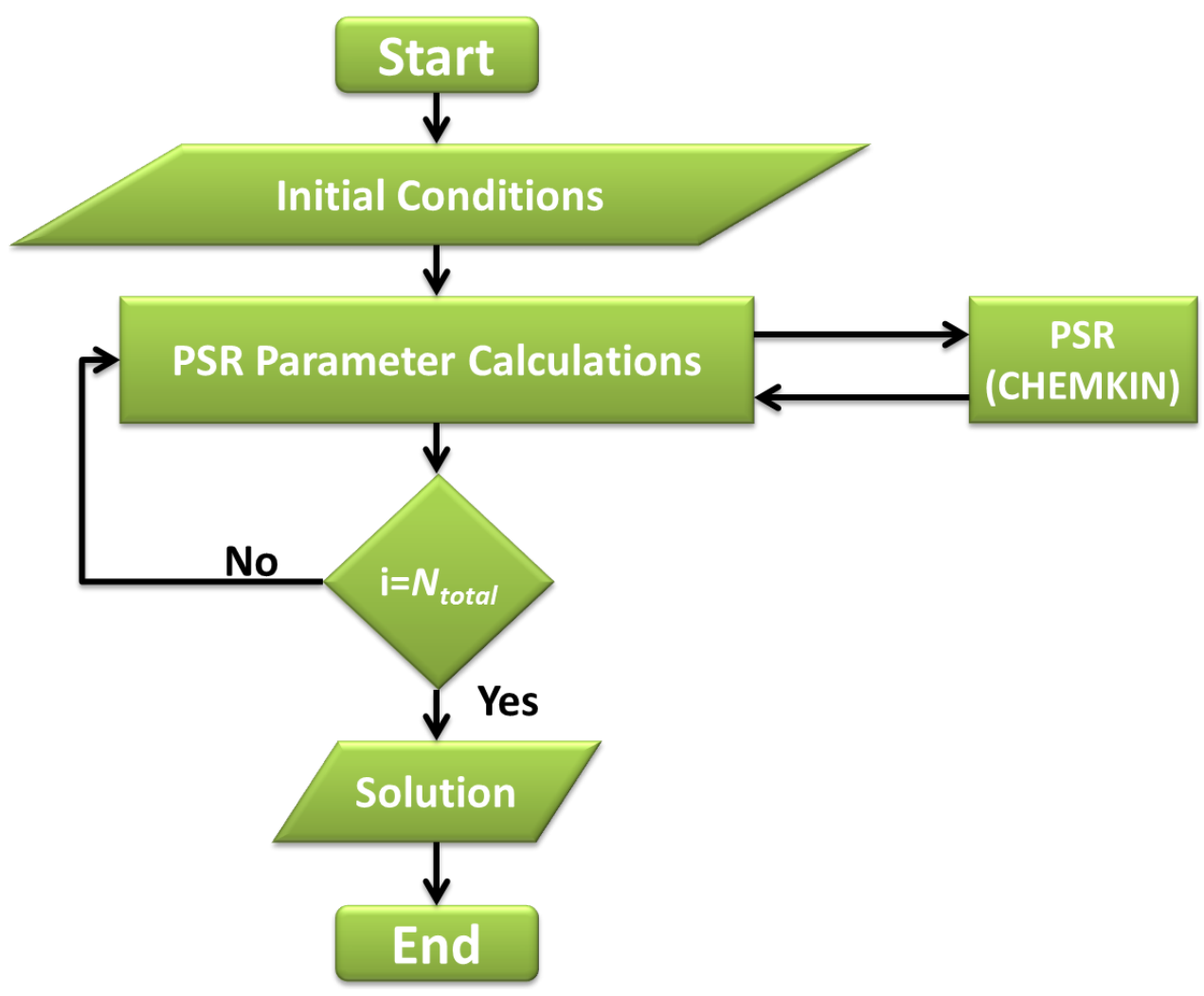

Figure 4.8. PFR Driver Flow Diagram. 
$N_{\text {total }}$ is the total number of PSRs that were used to simulate the PFR domain. In order to correctly represent the PFR it is necessary to use several PSRs in series. Since the PFR domain is discretized in several PSR regions the trend of the predicted species concentration should converge to the PFR profile as the number of PSRs $N_{\text {total }} \rightarrow \infty$.

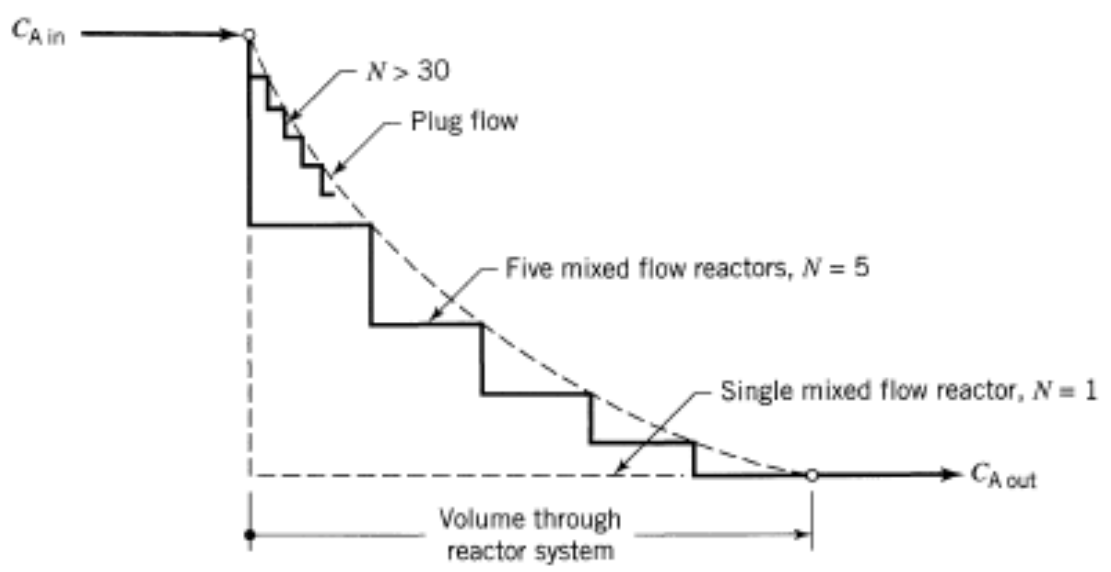

Figure 4.9. PFR Approximation Using Series of PSRs (Levenspiel 1962).

Figure 4.9 shows the trend comparison of a $A$ specie using the current approach. In order to approximate the PFR with a finite number of PSRs a "reactor independence" study was performed. The study consists in monitoring species concentrations at the outlet of the domain and verify that further increase in $N_{\text {total }}$ does not significantly affect the predicted concentrations (less than 5\% change).

\subsection{CRN Results}

\subsubsection{Domain Decomposition - NETL Burner}

Results from CFD simulations were loaded in the CRN-DIV program. The computational domain was divided according to two control variables; temperature and carbon dioxide mass fraction. These two variables were selected because they help to characterize the burner zones in regions such as inlet, flame, recirculation, and post-flame. The CRN was created using CFD simulation results from the simplest chemical mechanism, i.e. a one step global reaction that 
contains five chemical species $\left(\mathrm{CH}_{4}, \mathrm{O}_{2}, \mathrm{CO}_{2}, \mathrm{H}_{2} \mathrm{O}, \mathrm{N}_{2}\right)$. The contours of the control variables are shown in Figure 4.10.

a)

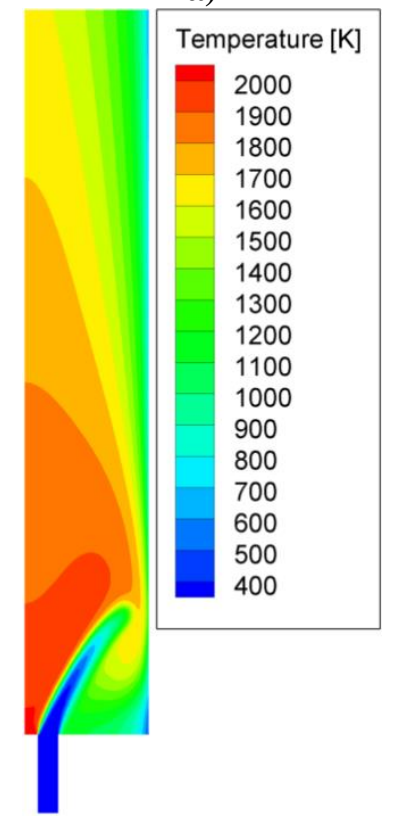

b)

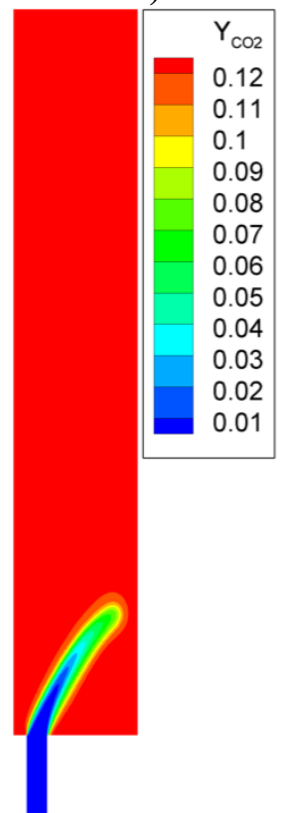

c)

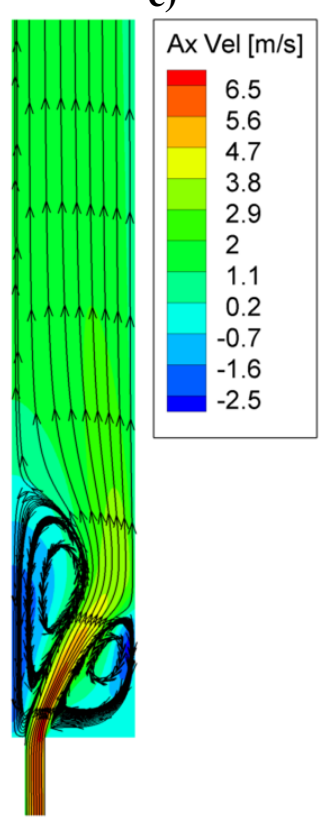

Figure 4.10. Confined Premixed Burner Predictions. a) Temperature [K] Contours, b) Mass Fraction of Carbon Dioxide Contours, c) Axial Velocity [m/s] Contours and Streamlines.

Equally spaced levels were chosen for the temperature and carbon dioxide control variables. Streamlines (Figure $4.10 \mathrm{c}$ )) show that the flow becomes one directional after the main recirculation zone allowing the implementation of a PFR model in this region. The rest of the reactors were marked as PSRs because the flow in these reactors is not clearly unidirectional. The id_cell contours are shown in Figure 4.11. 
a)

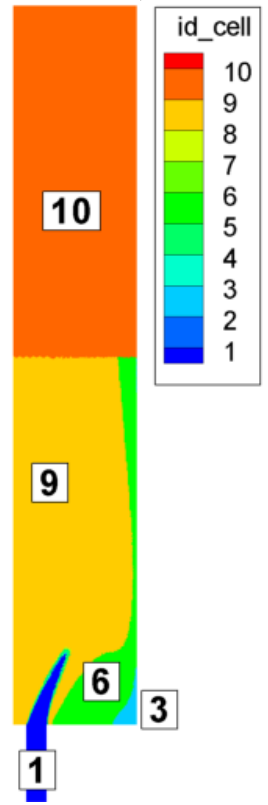

b)

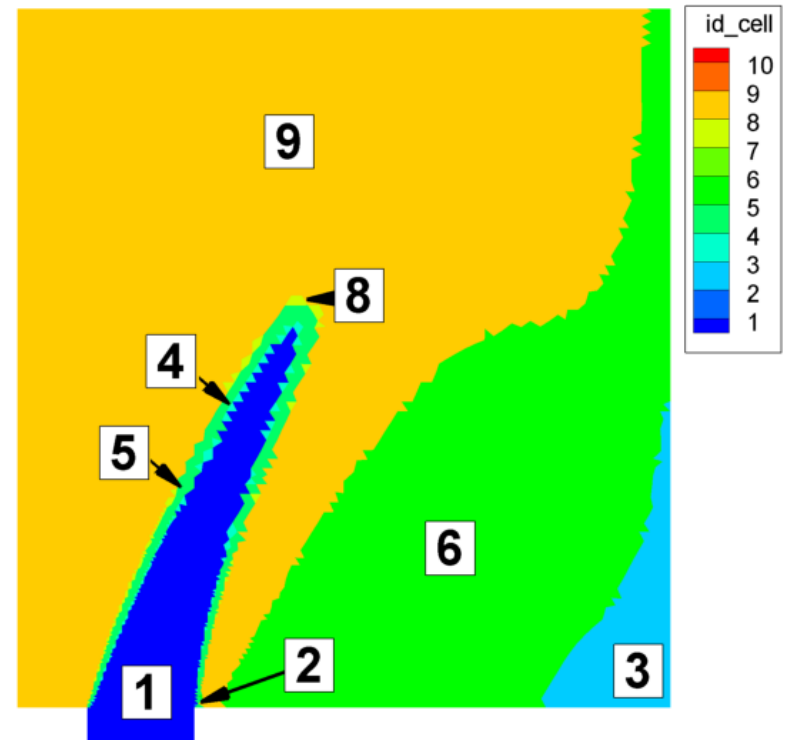

Figure 4.11. ID Cell Contours of the NETL Burner using CFD with Global Mechanism Data. a) Complete Domain, b) Flame Region.

The created CRN consisted of ten reactors. The average temperature, average composition and reactor volume were calculated using the CRN-DIV code. 


\subsubsection{CRN Results - NETL Burner}

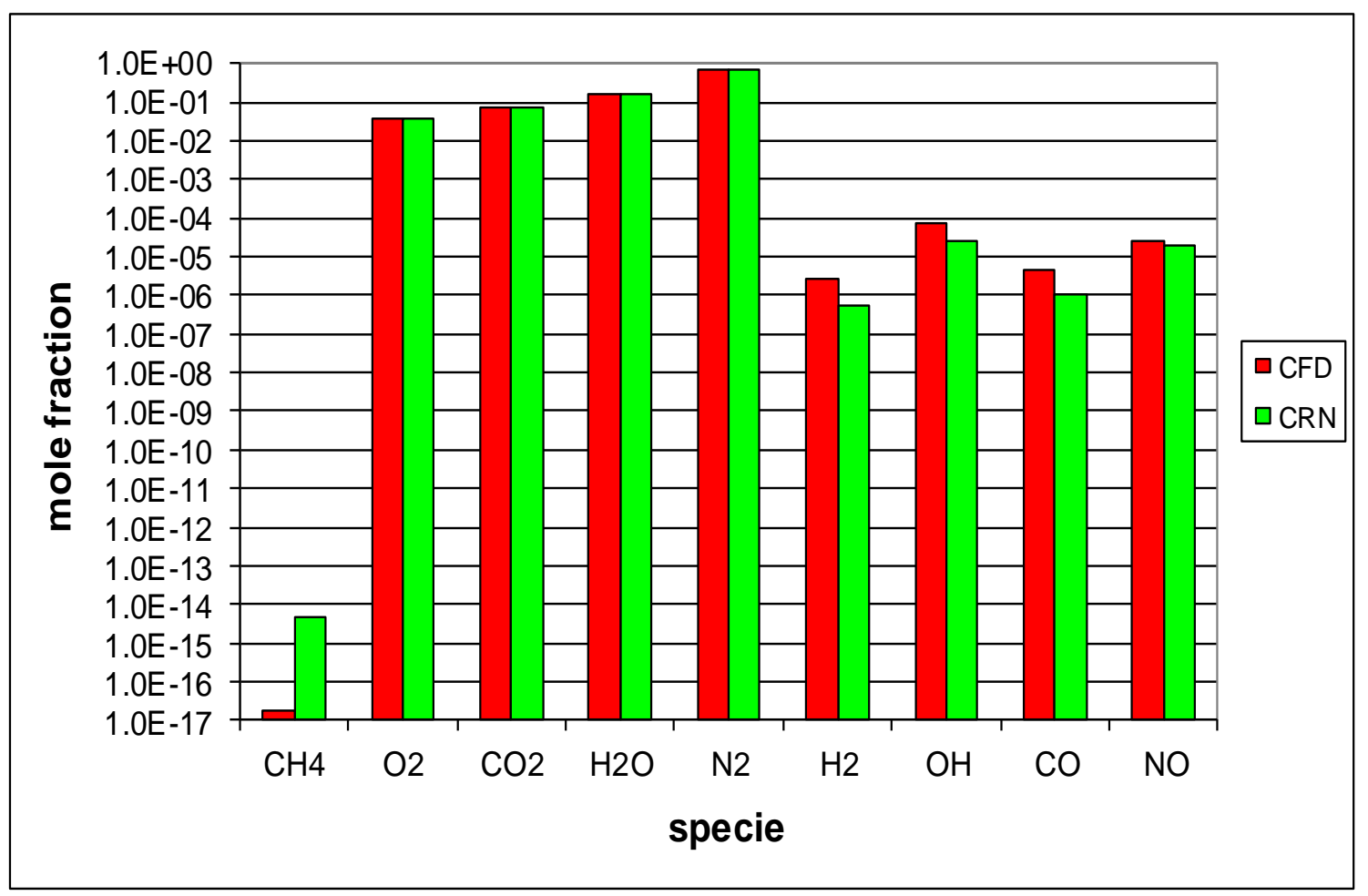

Figure 4.12. Outlet Mole Fractions. CRN GRI 3.0, CFD ARM9.

Figure 4.12 shows the results obtained using the CRN method with the detailed chemistry. The CFD input data used in the CRN calculation was obtained using the one step global mechanism. CFD results showed in Figure 4.12 were obtained using the ARM9 mechanism. CRN results are close to the CFD simulation values as it can be seen in Figure 4.12. The difference in concentration of the $\mathrm{CRN}$ results can be attributed to the fact that the composition in the combustor involves more chemical species while keeping the mass in the reactor the same. That is the summation of the mole fraction must be equal to one. Table 4.1 shows the numerical values of the species shown in Figure 4.12. 
Table 4.1. Mole Fraction Comparison. CFD ARM9, CRN GRI 3.0.

\begin{tabular}{|c|c|c|}
\hline Specie & CFD ARM9 & CRN GRI 3.0 <- CFD + glob. mech. \\
\hline $\mathbf{C H}_{\mathbf{4}}$ & $1.745 \mathrm{E}-17$ & $4.470 \mathrm{E}-15$ \\
\hline $\mathbf{O}_{\mathbf{2}}$ & $3.880 \mathrm{E}-02$ & $3.870 \mathrm{E}-02$ \\
\hline $\mathbf{C O}_{\mathbf{2}}$ & $7.729 \mathrm{E}-02$ & $7.770 \mathrm{E}-02$ \\
\hline $\mathbf{H}_{\mathbf{2}} \mathbf{O}$ & $1.550 \mathrm{E}-01$ \\
\hline $\mathbf{N}_{\mathbf{2}}$ & $1.553 \mathrm{E}-01$ & $7.280 \mathrm{E}-01$ \\
\hline $\mathbf{H}_{\mathbf{2}}$ & $7.285 \mathrm{E}-01$ & $5.530 \mathrm{E}-07$ \\
\hline $\mathbf{O H}$ & $2.721 \mathrm{E}-06$ & $2.720 \mathrm{E}-05$ \\
\hline $\mathbf{C O}$ & $7.559 \mathrm{E}-05$ & $1.050 \mathrm{E}-06$ \\
\hline $\mathbf{N O}$ & $4.480 \mathrm{E}-06$ & $2.020 \mathrm{E}-05$ \\
\hline
\end{tabular}

Mole fractions calculated from CRN match closely the CFD results of primary or major species such as; $\mathrm{O}_{2}, \mathrm{CO}_{2}, \mathrm{H}_{2} \mathrm{O}$, and $\mathrm{N}_{2}$. Differences in the calculated values are relatively larger for $\mathrm{H}_{2}, \mathrm{OH}, \mathrm{CO}$, and NO. But even when the difference is seemingly significant, the absolute numbers show that the difference in the CO mole fraction is $\sim 3.4 \times 10^{-6}(3.4[\mathrm{ppm}])$ and for NO is $\sim 6 \times 10^{-6}(6$ [ppm]). The predicted results using the CRN and CFD approaches are fairly similar. Another important aspect of the CRN is that it provides a concentration value for the extra chemical species that are not available in the CFD simulations. The extra species are shown in Table 4.2.

Table 4.2. CRN Extra Species. CFD ARM9, CRN GRI 3.0.

\begin{tabular}{|c|c|c|c|c|c|}
\hline $\mathbf{H}$ & $6.240 \mathrm{E}-09$ & $\mathbf{N}$ & $1.310 \mathrm{E}-15$ & HCNO & $6.180 \mathrm{E}-09$ \\
\hline $\mathbf{O}$ & $3.050 \mathrm{E}-07$ & $\mathbf{N H}$ & $1.190 \mathrm{E}-13$ & HOCN & $5.220 \mathrm{E}-10$ \\
\hline $\mathbf{H O}_{\mathbf{2}}$ & $4.310 \mathrm{E}-08$ & $\mathbf{N H}_{\mathbf{2}}$ & $2.090 \mathrm{E}-11$ & $\mathbf{H N C O}$ & $2.820 \mathrm{E}-09$ \\
\hline $\mathbf{H}_{\mathbf{2}} \mathbf{O}_{\mathbf{2}}$ & $1.020 \mathrm{E}-08$ & $\mathbf{N H}_{\mathbf{3}}$ & $1.010 \mathrm{E}-09$ & $\mathbf{N C O}$ & $4.430 \mathrm{E}-12$ \\
\hline $\mathbf{C H}_{\mathbf{3}}$ & $9.620 \mathrm{E}-16$ & $\mathbf{N N H}$ & $5.490 \mathrm{E}-15$ & & \\
\hline $\mathbf{C H}_{\mathbf{2}} \mathbf{O}$ & $1.840 \mathrm{E}-15$ & $\mathbf{N O}_{\mathbf{2}}$ & $9.550 \mathrm{E}-08$ & & \\
\hline $\mathbf{C H}_{\mathbf{3}} \mathbf{O H}$ & $5.760 \mathrm{E}-16$ & $\mathbf{N}_{\mathbf{2}} \mathbf{O}$ & $1.860 \mathrm{E}-07$ & & \\
\hline $\mathbf{C}_{\mathbf{2}} \mathbf{H}_{\mathbf{2}}$ & $6.400 \mathrm{E}-13$ & $\mathbf{H N O}$ & $4.840 \mathrm{E}-12$ & & \\
\hline $\mathbf{C H}_{\mathbf{2}} \mathbf{C O}$ & $3.450 \mathrm{E}-14$ & $\mathbf{C N}$ & $7.410 \mathrm{E}-16$ & & \\
\hline $\mathbf{H C C O H}$ & $4.100 \mathrm{E}-11$ & $\mathbf{H C N}$ & $1.810 \mathrm{E}-10$ & & \\
\hline
\end{tabular}

The following chemical species were not included because their mole fraction was less than $1 \times 10^{-16}$ which falls outside the precision of the computer; $\mathrm{C}, \mathrm{CH}, \mathrm{CH}_{2}, \mathrm{CH}_{2}(\mathrm{~S}), \mathrm{HCO}, \mathrm{CH}_{2} \mathrm{OH}$, $\mathrm{CH}_{3} \mathrm{O}, \mathrm{C}_{2} \mathrm{H}, \mathrm{C}_{2} \mathrm{H}_{3}, \mathrm{C}_{2} \mathrm{H}_{4}, \mathrm{C}_{2} \mathrm{H}_{5}, \mathrm{C}_{2} \mathrm{H}_{6}, \mathrm{HCCO}, \mathrm{H}_{2} \mathrm{CN}, \mathrm{HCNN}, \mathrm{Ar}, \mathrm{C}_{3} \mathrm{H}_{7}, \mathrm{C}_{3} \mathrm{H}_{8}, \mathrm{CH}_{2} \mathrm{CHO}, \mathrm{CH}_{3} \mathrm{CHO}$.

In order to assess the results of the accuracy of the CRN results they were compared to the CFD results which used the ARM19 mechanism. 


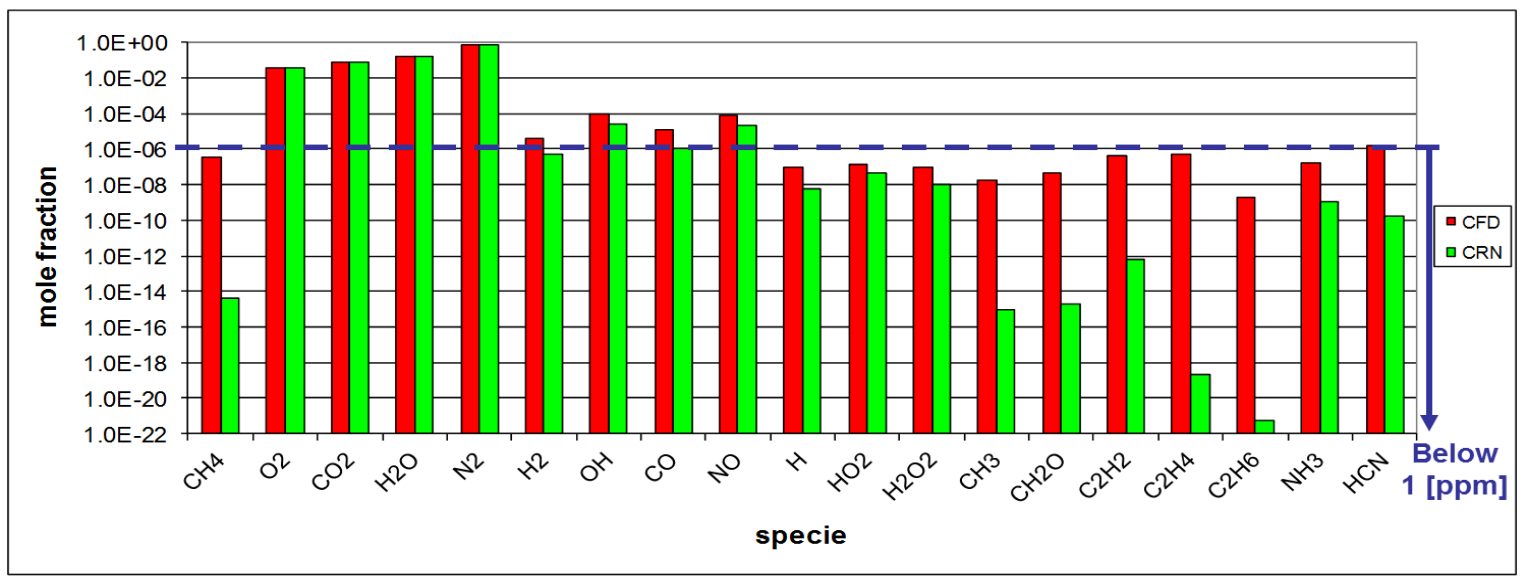

Figure 4.13. Outlet Mole Fractions. CRN GRI 3.0, CFD ARM19.

Figure 4.13 shows that the CFD ARM19 results are relatively close to the CRN results when the specie concentration is above the $1[\mathrm{ppm}]$ level $\left(1 \times 10^{-6}\right)$, when the specie concentration is lower than the $1[\mathrm{ppm}]$ level difference becomes significant. Table 4.3 contains the numerical values of the concentrations shown in Figure 4.13.

Table 4.3. Mole Fractions Comparison. CFD ARM19, CRN GRI 3.0.

\begin{tabular}{|c|c|c|}
\hline Specie & CFD ARM19 & CRN GRI 3.0 <- CFD + glob. mech. \\
\hline $\mathbf{C H}_{\mathbf{4}}$ & $3.427 \mathrm{E}-07$ & $4.470 \mathrm{E}-15$ \\
\hline $\mathbf{O}_{\mathbf{2}}$ & $3.764 \mathrm{E}-02$ & $3.870 \mathrm{E}-02$ \\
\hline $\mathbf{C O}_{\mathbf{2}}$ & $7.842 \mathrm{E}-02$ & $7.770 \mathrm{E}-02$ \\
\hline $\mathbf{H}_{\mathbf{2}} \mathbf{O}$ & $1.550 \mathrm{E}-01$ \\
\hline $\mathbf{N}_{\mathbf{2}}$ & $1.565 \mathrm{E}-01$ & $7.280 \mathrm{E}-01$ \\
\hline $\mathbf{H}_{\mathbf{2}}$ & $7.273 \mathrm{E}-01$ & $5.530 \mathrm{E}-07$ \\
\hline $\mathbf{O H}$ & $3.877 \mathrm{E}-06$ & $2.720 \mathrm{E}-05$ \\
\hline $\mathbf{C O}$ & $8.925 \mathrm{E}-05$ & $1.050 \mathrm{E}-06$ \\
\hline $\mathbf{N O}$ & $1.252 \mathrm{E}-05$ & $2.020 \mathrm{E}-05$ \\
\hline $\mathbf{H}$ & $8.237 \mathrm{E}-05$ & $6.240 \mathrm{E}-09$ \\
\hline $\mathbf{H O}$ & $9.766 \mathrm{E}-08$ & $4.310 \mathrm{E}-08$ \\
\hline $\mathbf{H}_{\mathbf{2}} \mathbf{O}_{\mathbf{2}}$ & $1.365 \mathrm{E}-07$ & $1.020 \mathrm{E}-08$ \\
\hline $\mathbf{C H}_{\mathbf{2}}$ & $8.730 \mathrm{E}-08$ & $6.400 \mathrm{E}-13$ \\
\hline $\mathbf{C H}_{\mathbf{2}} \mathbf{O}$ & $1.663 \mathrm{E}-08$ & $1.840 \mathrm{E}-15$ \\
\hline $\mathbf{C}_{\mathbf{2}} \mathbf{H}_{\mathbf{2}}$ & $4.445 \mathrm{E}-08$ & $6.400 \mathrm{E}-13$ \\
\hline $\mathbf{C}_{\mathbf{2}} \mathbf{H}_{\mathbf{4}}$ & $4.380 \mathrm{E}-07$ & $2.24 \mathrm{E}-19$ \\
\hline $\mathbf{C}_{\mathbf{2}} \mathbf{H}_{\mathbf{6}}$ & $5.452 \mathrm{E}-07$ & $5.61 \mathrm{E}-22$ \\
\hline $\mathbf{N H}_{\mathbf{3}}$ & $1.944 \mathrm{E}-09$ & $1.01 \mathrm{E}-09$ \\
\hline $\mathbf{H C N}$ & $1.708 \mathrm{E}-07$ & $1.81 \mathrm{E}-10$ \\
\hline
\end{tabular}

Comparison between the CRN and CFD ARM19 shown in Table 4.3 follows the same trend as Table 4.1. Predicted mole fraction of species; $\mathrm{O}_{2}, \mathrm{CO}_{2}, \mathrm{H}_{2} \mathrm{O}$, and $\mathrm{N}_{2}$ is similar for both 
approaches but the difference becomes relatively significant for the rest of the species. The concentration obtained from CRN was one tenth smaller than the CFD predicted value for $\mathrm{CO}$ and one fourth smaller than the CFD prediction for NO.

\section{Computational Time}

The aforementioned calculations were performed in the CFD AMP computational cluster. The cluster is composed of 64 Intel Xeon processors each @ $2.33[\mathrm{GHz}]$. CFD simulations were always performed in parallel which means that the workload of the calculations was divided in several processors, and for the CRN the calculation were always performed in one processor (serial calculation). The calculation time is shown in Table 4.4.

Table 4.4. Calculation Time for Case 1 NETL Burner.

\begin{tabular}{|c|c|c|}
\hline Case & Number of Processors & Real Time [hrs] \\
\hline CFD with glob. mech. & 8 & 3.44 \\
\hline CFD with ARM9 & 4 & 30.13 \\
\hline CFD with ARM9 + CFD with ARM19 & 4 & $30.13+75.4=105.53$ \\
\hline CFD with glob. mech. + CRN GRI 3.0 & 1 & $3.44+0.3=3.74$ \\
\hline
\end{tabular}

The real time column in Table 4.4 is the actual time that took the computer to complete the calculation of the problem. It was not needed to parallelize the CRN program because the number of reactors is considerably less than the number of computational cells in the CFD simulations. CFD calculations were performed in eight or four processors due the availability of computer resources at the time when calculations were computed. Due the non-linearity present in the ARM19 it is necessary to start the calculations with a good initial condition otherwise the simulation could diverge or the flame could extinguish. The CFD simulations with ARM19 took 75.4 [hrs] to complete but because the ARM9 was used as the initial condition the time that took for the ARM9 solution to converge was added to the ARM19 calculations. In the same fashion the CRN calculations needed the solution of the CFD global reaction in order to have the necessary information to build the reactor network so the time needed to the CFD with global mechanism to converge was added to the CRN calculations. Table 4.4 shows that the $\mathrm{CRN}$ calculations needed a 
tenth of the real time of the CFD ARM9 simulation and around one twenty eighth of the real time of the CFD ARM19 simulation. But the time comparison using the real time and not taking into account the parallelization of the calculations is unfair. One way to have a level ground for all calculations is to perform calculations using the same number of processors, this implies that the CRN will have to be written in parallel. CRN parallelization is outside of the scope of the current study and might be pursued in the future. Another option will be to run all calculations in just one processor but the time required to complete the calculations will be too long and possible processor overload issues might arise. Instead Amdahl's law (Gustafson 1988) was used to convert the real time needed by the parallel simulations to the time it will be required to complete the same calculations using only one processor. Amdahl's law is used to estimate how much the computer calculations will speedup if parts of the program were parallelized as a function of the number of processors. The speedup is calculated using Equation 4.9.

speedup $=\frac{s+p}{s+p / N}$

Where $s$ is the percentage of the code that is computed in series and $p$ is the percentage calculated using parallelization, $N$ is the number of processors used. For simplicity the total time is set to 1 $(s+p=1)$. Assuming that the percentage of the FLUENT code works in parallel is $95 \%(p=0.95)$ because the parts of the code that needed to be serial are basically to read the boundary conditions and the operational conditions, the majority of the work is usually performed in parallel (coefficient calculation, solution loop, etc.). With 95\% percentage the calculated speedup using the Amdhal's law as a function of the number of processors is shown in Figure 4.14. 


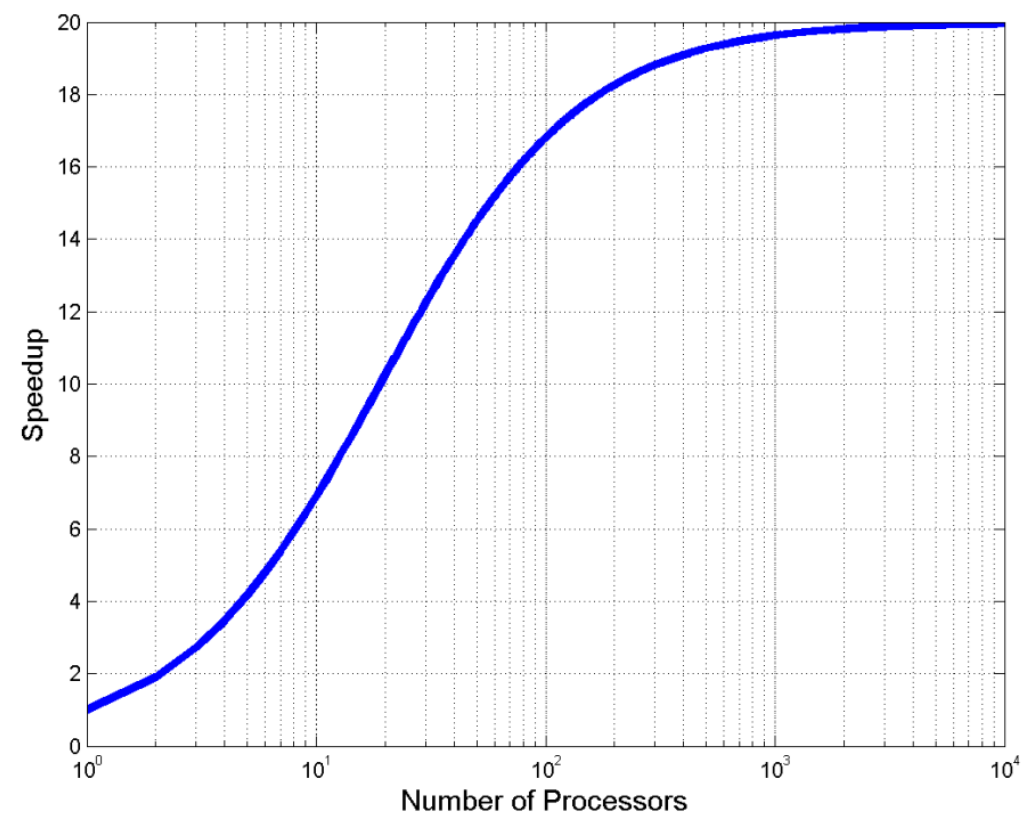

Figure 4.14. Parallel Speedup Calculation Using Amdhal's Law.

Figure 4.14 shows that the maximum speed up for a code with $95 \%$ in parallel is 20 with respect to the serial version. Using the data for four and eight processors it was found that the speedup is 3.47 and 5.92 respectively, the speedup was multiplied by the real time from Table 4.4 and the time of the calculation as if it was performed in just one processor was found.

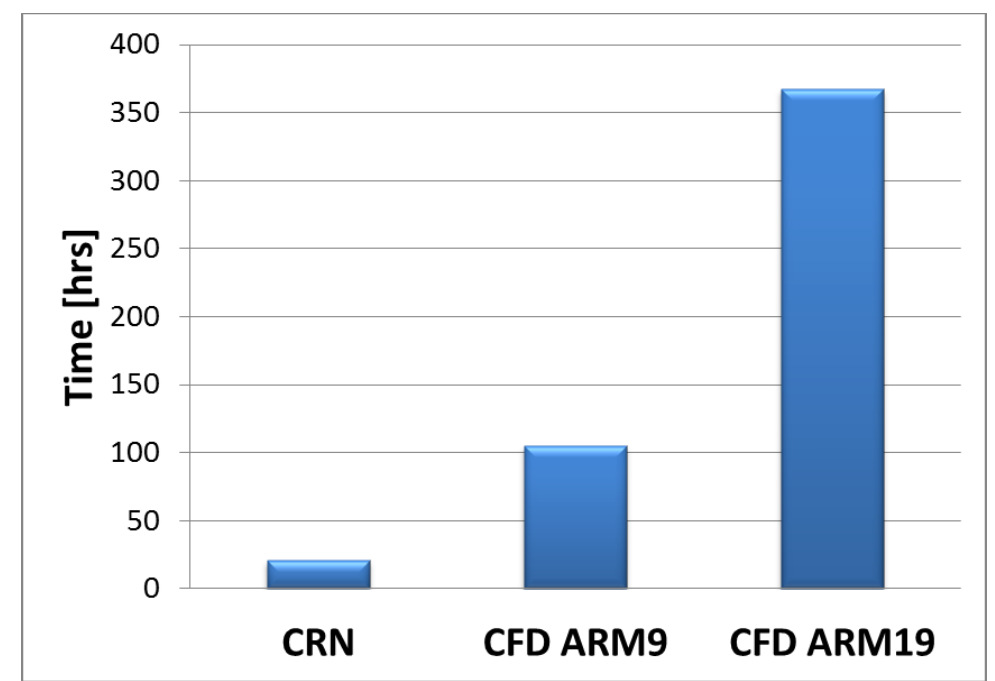

Figure 4.15. Serial CPU Time. 
Results showed that the time needed to complete the simulation using the CRN approach is one fifth of the time required for the CFD ARM9 calculations and one seventeenth of the time required for the CFD ARM19 calculations.

It must be stressed that the calculation of the serial time is merely an approximation because the exact percentage of the ANSYS-FLUENT software that works in serial when the parallel option is activated is not known. Moreover there are other hardware related bottle necks that can affect the speedup. In this regard the results in Figure 4.15 should be viewed as an informed estimation.

CRN calculations had shown to be faster than CFD with more complete chemical mechanisms. In order to assess the accuracy of the calculations with experiments another problem setup was used because no species concentration measurements were available for the current case at the time of the calculation. Plus it provided the opportunity to test the $\mathrm{CRN}$ for a different burner configuration.

\subsubsection{Domain Decomposition - TECFLAM Burner}

The CRN approach was also applied for the non-premixed TECFLAM burner (Landenfeld, et al. 1998). The CRN-DIV program used as input the results from the CFD with a two step global mechanism simulations presented in Section 3.2. Temperature and water mass fraction were used as control variables to create the reactor. CFD results of the temperature and water mass fraction are shown in Figures 4.16 a) and b) for the entire burner domain. Temperature and mass fraction were selected as control variables because it was clear to separate the burner in several characteristic regions namely; flame, mixing, and post flame regions. Due to the nature of the flow all the reactors created by in the CRN-DIV program were marked as PSRs because it was observed that the flow did not form a region where it is clearly one dimensional. Figure $4.16 \mathrm{c}$ ) shows the streamlines and the axial velocity contours of the flow inside the domain. Besides the recirculation zones located at 
the flame region and on the side wall there are two extra recirculations. These recirculations appear due the constriction of the outlet by the top wall. The appearance of additional recirculation zones does not allow the flow in the post-flame region to become unidirectional as in the previous burner (NETL burner). For this reason all of the reactors in the CRN calculations were identified as PSRs. Nevertheless, additional calculations of the reactor network were performed where the region near the outlet was marked as a PFR but these simulations produced unsatisfactory results when compared with the experiments.

a)

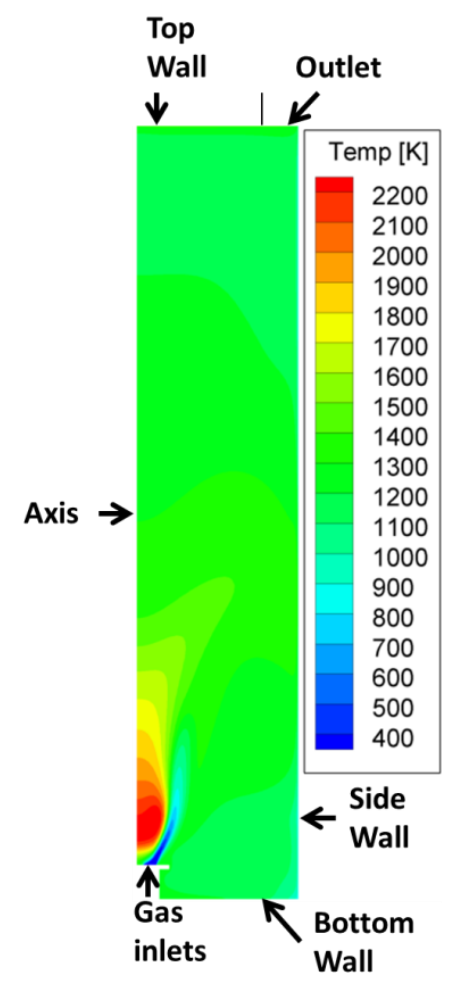

b)

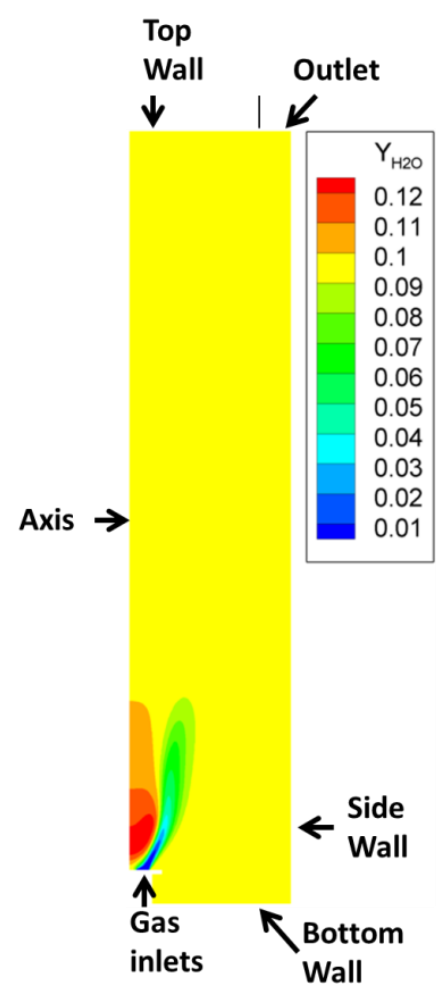

c)

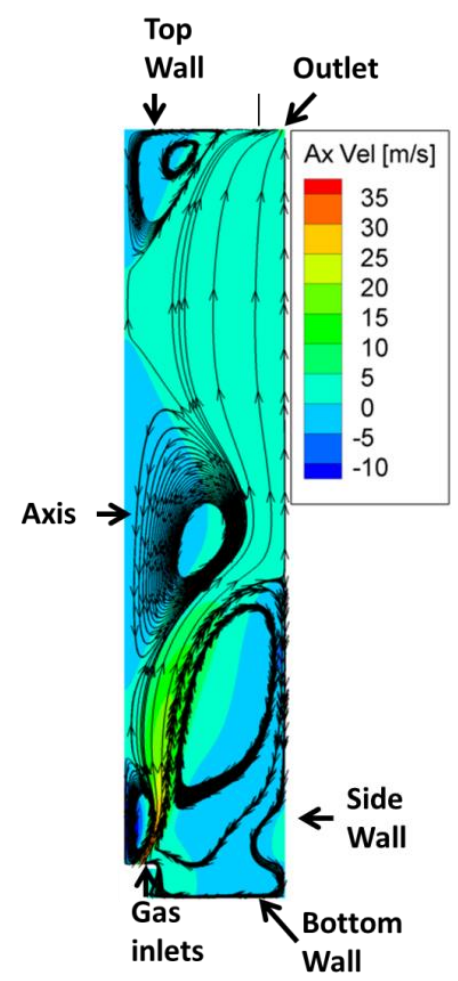

Figure 4.16. CFD Solution Contours TECFLAM Burner. a) Temperature [K] Contours, b) Water Mass Fraction Contours, c) Axial Velocity [m/s] Contours and Streamlines. 
a)

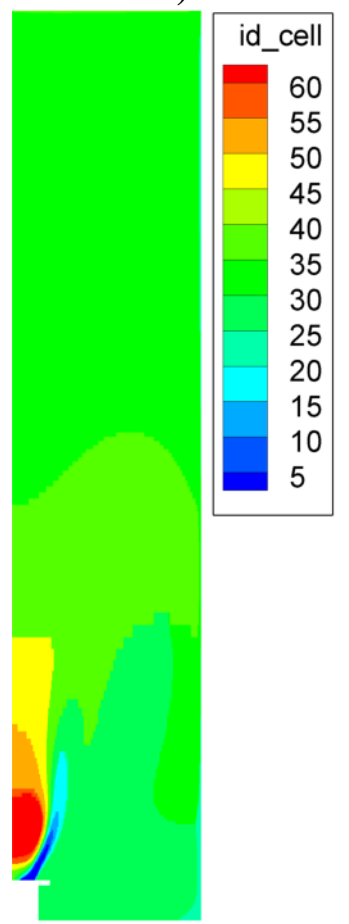

b)

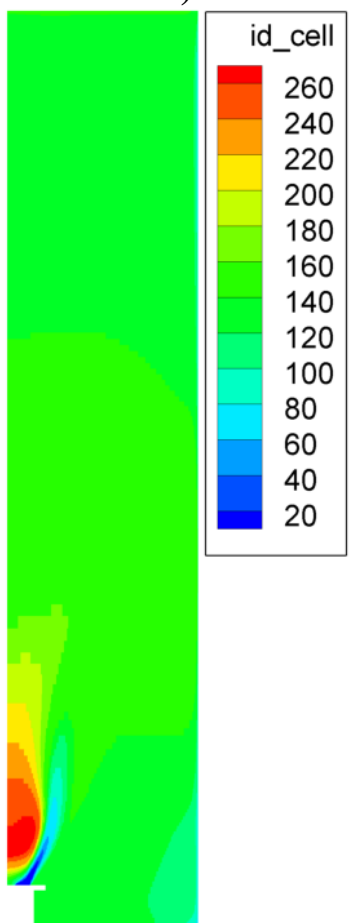

c)

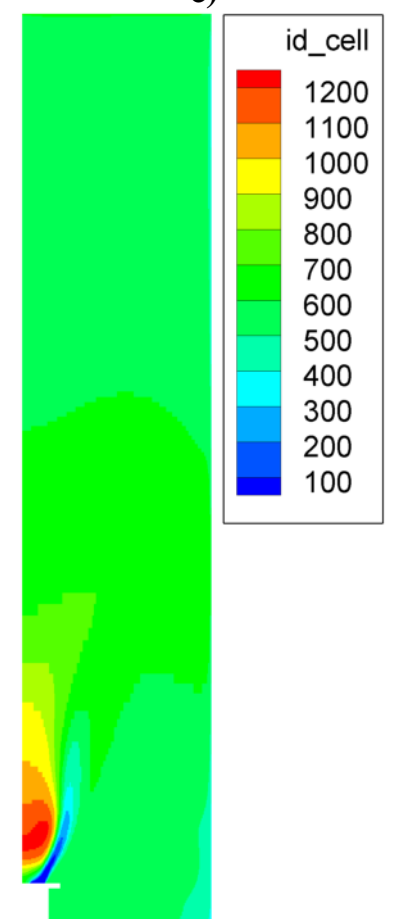

Figure 4.17. ID Cell Contours TECFLAM Burner. a) $n_{T e m p}=9, n_{H 2 O}=9$, b) $n_{T e m p}=18, n_{H 2 O}=18$, c) $n_{\text {Temp }}=36, n_{\mathrm{H} 2 \mathrm{O}}=36$.

Figure 4.17 shows the resulting zones when the levels of temperature and the water mass fraction variables were defined with nine levels. The total number of reactors were; 26, 79, and 268 respectively. The total number of reactors is less than the total number of combinations possible, $n_{\text {Temp }} * n_{H 2 O}$, because there were no computational cells that fell in some of the bins. Figure 4.17 shows that with increasing the number of bins the divided reactor resembles the temperature field from the CFD simulations.

\subsubsection{CRN Results - TECFLAM Burner}

\section{Contour Reconstruction}

CRN predictions of the TECFLAM burner were performed using the methane-air mechanism GRI 3.0. Results from the CRN simulation represent the concentration for a reactor in a specific region of the burner, this information was used to re-construct the burner domain in order 
to compare against the measured species mass fraction profiles. The contour reconstruction was performed using the mesh information, the id_cell value and the results from the CRN simulation. Contour reconstruction was performed by a MATLAB program that created the CFD mesh, then it looped through all the cells of that mesh and checked the id_cell value, finally the program looked for the CRN results of the id_cell and pasted them into the two dimensional mesh.

a)

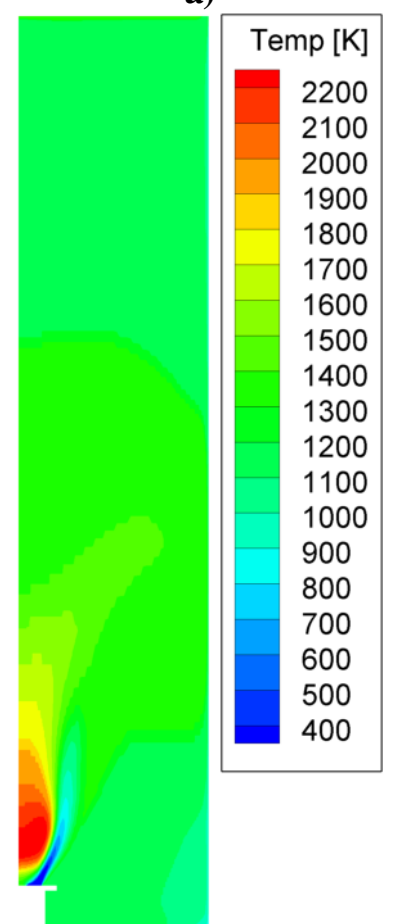

b)

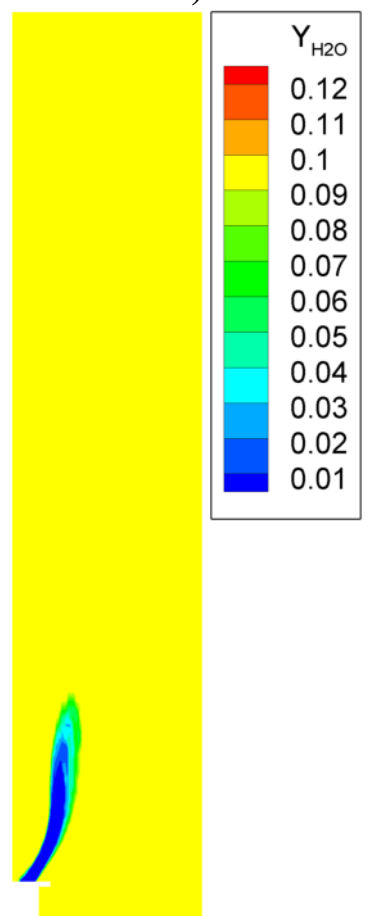

Figure 4.18. Contour Reconstruction using Results from CRN, $n_{\text {Temp }}=18$ and $n_{H 2 O}=18$. a) Temperature [K], b) Water Mass Fraction.

Figure 4.18 a) shows the temperature contour reconstruction using data from the CRN simulation $n_{\text {Temp }}=18$ and $n_{H 2 O}=18$. When Figure 4.18 a) is compared with 4.16 a) it can be seen that two contours matched very well, this means that the reconstruction was successful. Figure 4.18 b) shows the reconstruction of the water mass fraction contour when compared with $4.16 \mathrm{~b}$ ) the concentration of water mass fraction decreased at the central recirculation zone and in the post flame region. A detailed comparison of the CFD, CRN, and experiments is shown in Figure 4.19. The energy equation was not solved in the CRN simulations for this reason the CFD and CRN 
temperature values follow the same trend as shown in Figure 4.19. CFD simulations were performed using a two step global mechanism which contains the following chemical species; $\mathrm{CH}_{4}$, $\mathrm{O}_{2}, \mathrm{H}_{2} \mathrm{O}, \mathrm{CO}_{2}, \mathrm{CO}, \mathrm{N}_{2}$.
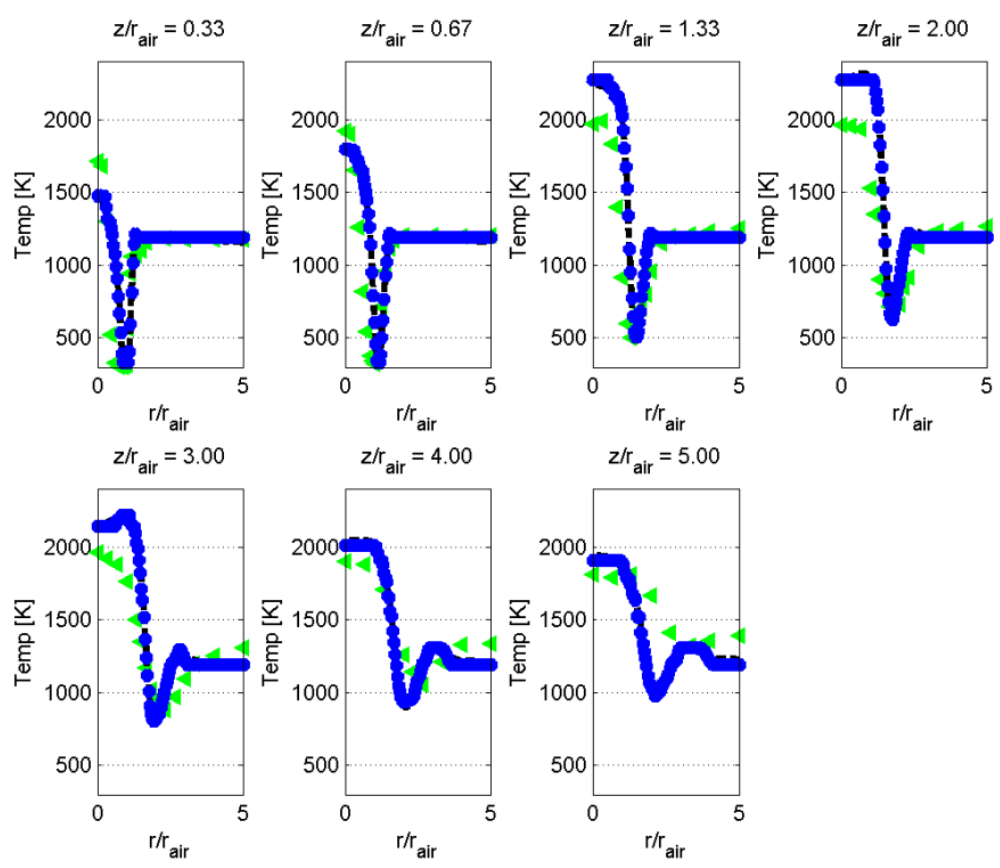

Figure 4.19. Temperature Profiles. Left Triangles - Experiments, Dash line - CFD Simulations with Two Step Global Mechanism, Blue Circles - CRN Simulations. 

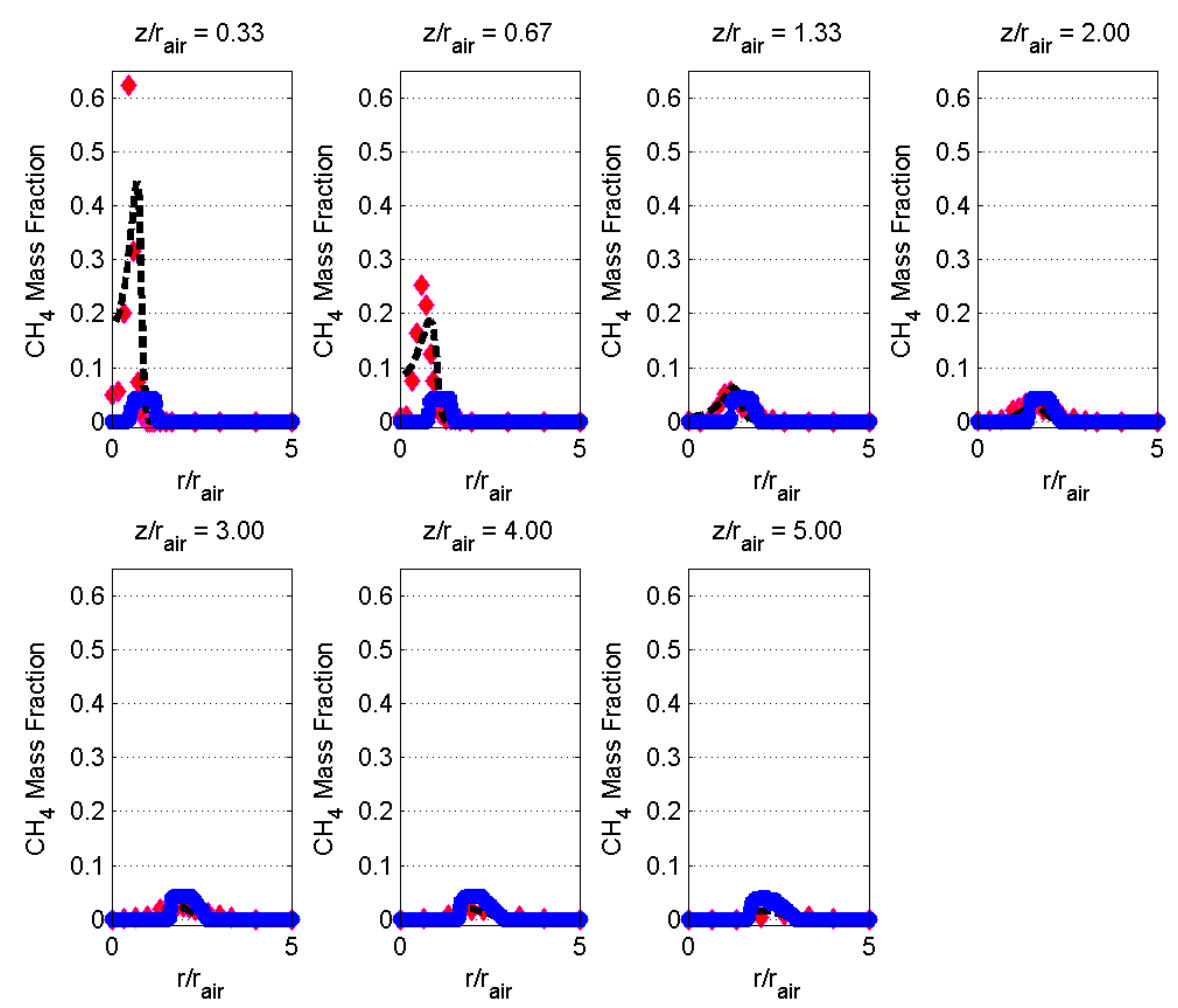

Figure 4.20. Methane Mass Fraction Profiles. Red Diamonds - Experiments, Dash line - CFD Simulations with Two Step Global Mechanism, Blue Circles - CRN Simulations.

CRN methane profiles at station $z / r_{\text {air }}=0.33$, and $z / r_{\text {air }}=0.67$ are lower than the experimental and the CFD results (Figure 4.20). The low concentration of methane is because the reactors obtained from the CRN division are relatively bigger than the CFD cells, the reactor that is located at the inlet of the domain covers both the methane and air inlets. The methane concentration near the dump plane is the average value of both air and methane inlets. The methane mass fractions in the subsequent stations where the combustion was establish, high temperature region, is closer to the measurements and the CFD simulations. 

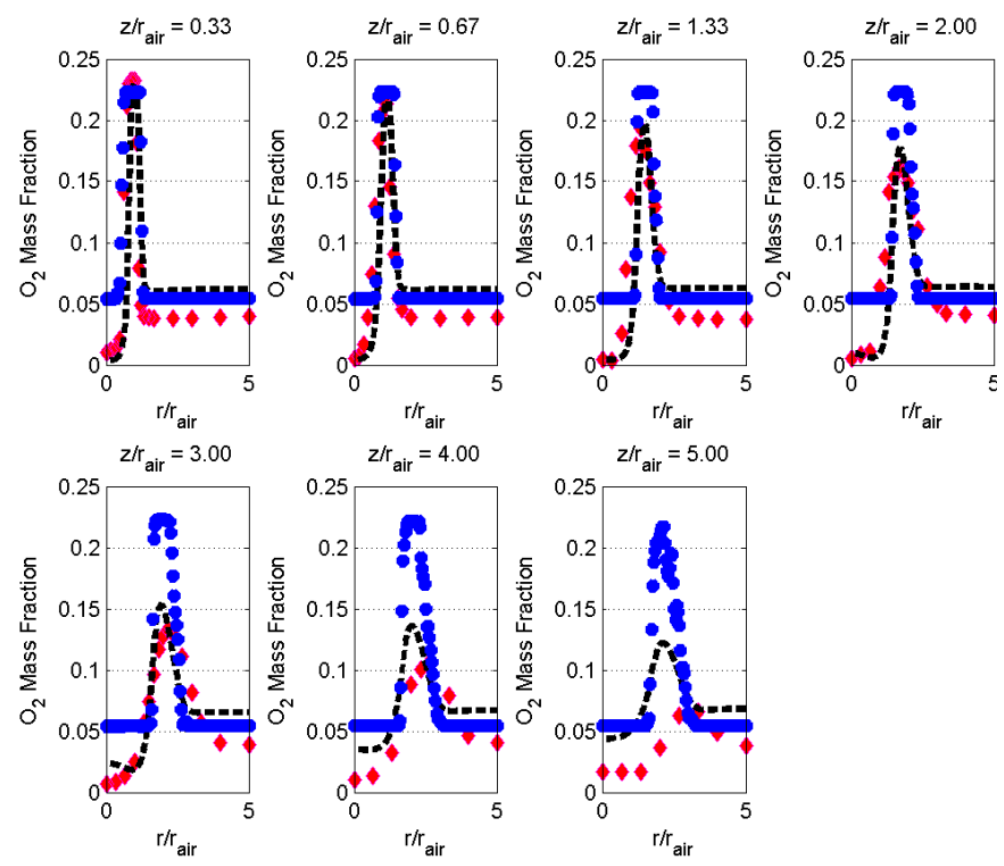

Figure 4.21. Oxygen Mass Fraction Profiles. Red Diamonds - Experiments, Dash line - CFD Simulations with Two Step Global Mechanism, Blue Circles - CRN Simulations.

The inlet value of the oxygen mass fraction in the CRN predictions extends longer than the experiments as shown in Figure 4.21. The extension of the inlet oxygen value deeper in the domain could be an indication that the reaction in the CRN calculations proceeds slower. On the other hand, CRN results are closer to the experiments when moving away from the axis, $r / r_{\text {air }}=5$. This region is close to a recirculation zone, recirculation zones have relatively large resident times that allow the reaction to proceed to completion. 

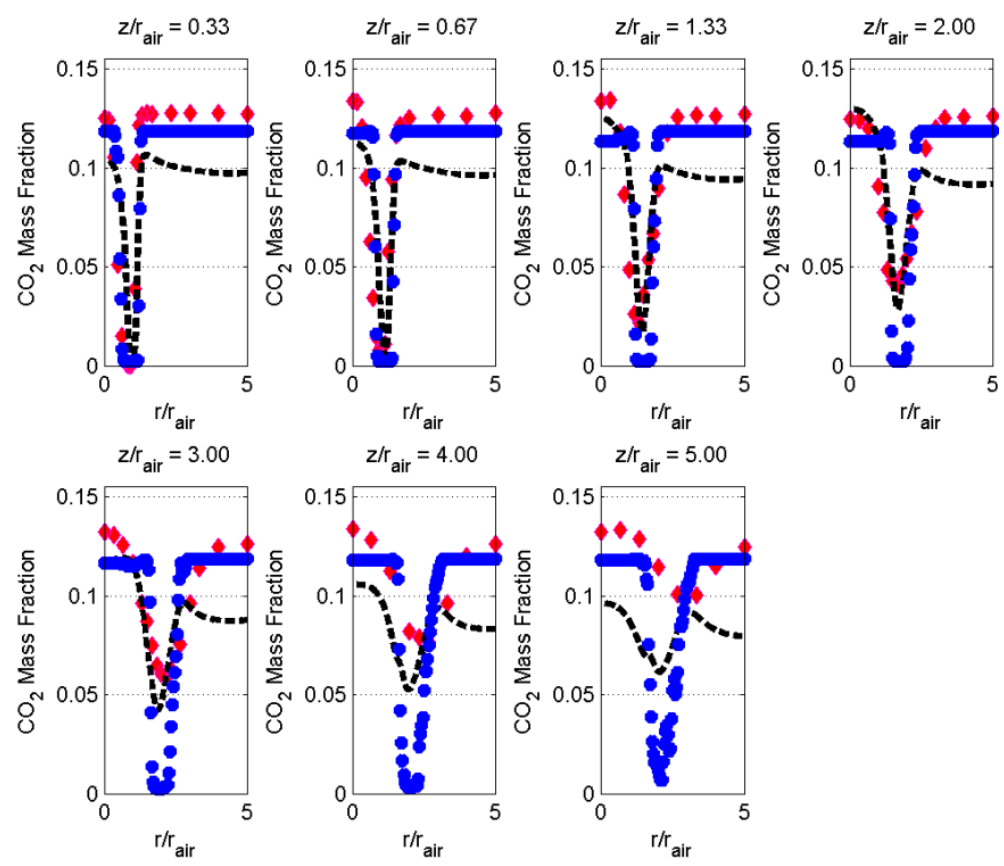

Figure 4.22. Carbon Dioxide Mass Fraction Profiles. Red Diamonds - Experiments, Dash line CFD Simulations with Two Step Global Mechanism, Blue Circles - CRN Simulations.

Predicted CRN carbon dioxide mass fractions are closer to the experiments than the CFD predictions as shown in Figure 4.22. However the CRN results also show that the spatial variation of the carbon dioxide concentration is lower than what was measured following a similar trend as the oxygen concentrations. 

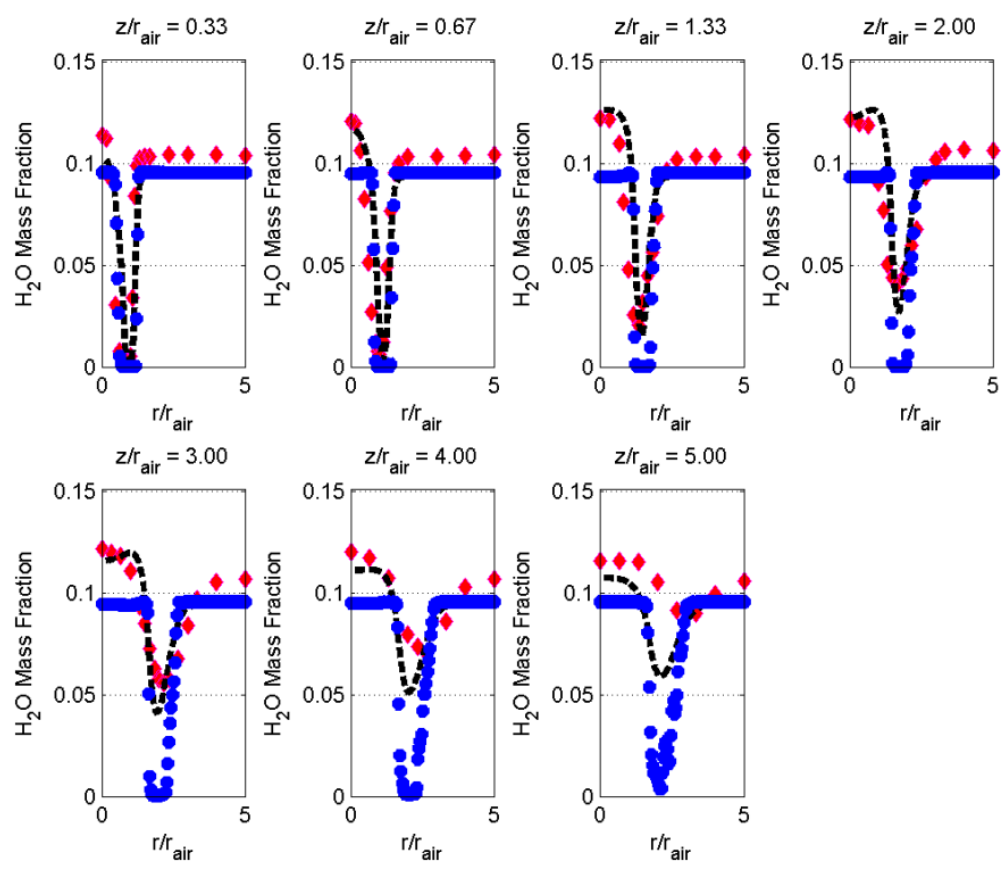

Figure 4.23. Water Mass Fraction Profiles. Red Diamonds - Experiments, Dash line - CFD Simulations with Two Step Global Mechanism, Blue Circles - CRN Simulations.

Water mass fractions calculated using the CRN show (Figure 4.23) a similar value in the regions, $r / r_{\text {air }}=0$ and $r / r_{\text {air }}=5$; this value is closer to the concentration at adiabatic flame condition at this equivalence ratio $Y_{H 2 O \text {,ad_temp }}=0.1$. The same lower variation of the mass fraction is seen for the region that is affected by the inlet mixture. The relatively large size of the CRN reactors meant that the locations near or in the recirculation zones were biased to the conditions where the residence time is large enough that the reactions proceed to near completion. 

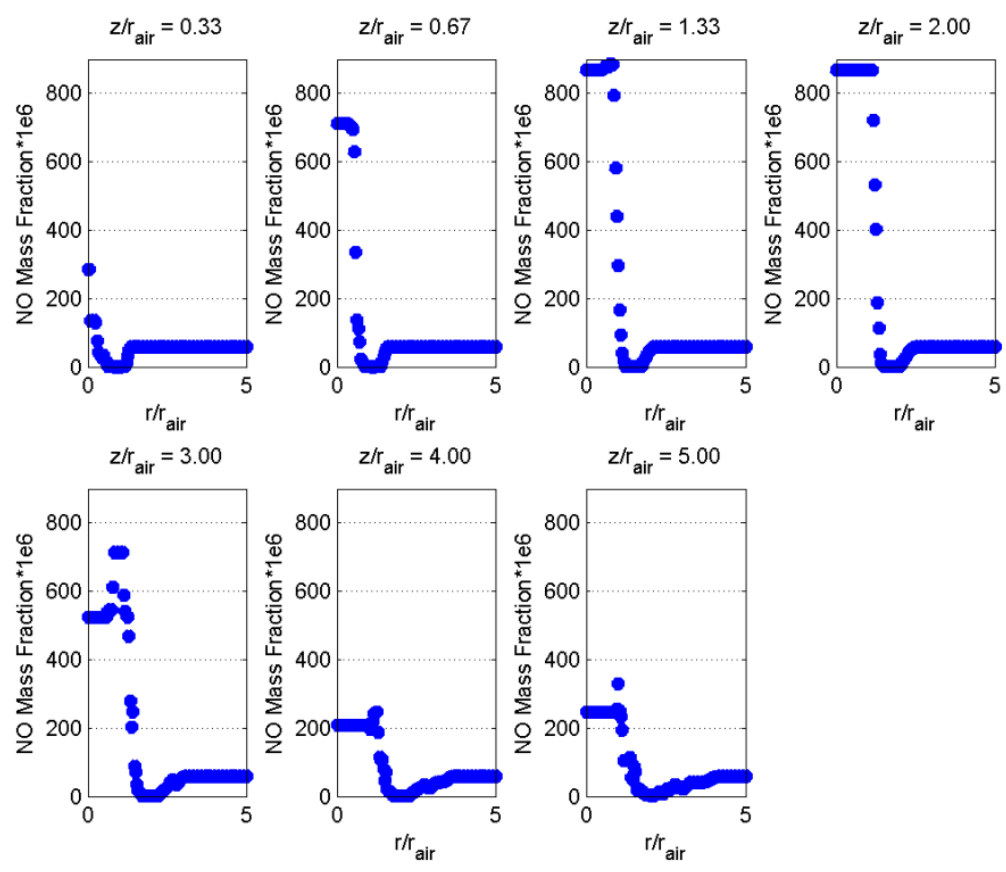

Figure 4.24. Nitrogen Oxide Mass Fraction Profiles. Blue Circles - CRN Simulations.

A feature of the CRN is the availability of extra species that are very difficult to measure experimentally or that were not included in the chemistry model used in CFD simulations because of the computing restrictions. Figure 4.24 shows the reconstructed profile of NO calculated using the CRN approach. The experiments did not include the profile measurements of NO; only the average value of $\mathrm{NO}$ at the outlet of the domain was reported. CFD calculations were performed with a two step global mechanism that did not include NO calculation either. Other reaction mechanisms include the NO calculation like the ARM9, however this mechanism was optimized for premixed combustion and could not be used for this non-premixed problem. Nevertheless in the current study a simulation using the ARM9 mechanism was performed but it was not possible to obtain a converged solution.

Reconstructed profiles using the CRN must be used with caution. It is necessary to remember that these profiles are defined in relatively large zones or reactors. Profiles can be informative but they are averaged values, in order to have an accurate representation it will be 
necessary to use more reactors. But using more reactors basically will take the total number of CRN reactors to the same scale as the computational cells used in CFD. A large number of reactors will increase the time of the calculation making the $\mathrm{CRN}$ approach computationally more expensive than the CFD simulation.

\section{Effect of the Number of Reactors}

In order to assess the effect of the number of reactors used in CRN several cases were simulated. The number of reactors was increased and the area averaged NO concentration was calculated and compared with the reported experimental value (Schmittel, et al. 2000). Table 4.5 shows the cases used to performed the calculations, all cases used equally spaced control variable levels calculated using Equation 4.1.

Table 4.5. CRN Cases for TECFLAM Burner.

\begin{tabular}{|c|c|c|c|}
\hline Case & $\boldsymbol{n}_{\text {Temperature }}$ & $\boldsymbol{n}_{\text {H2O }}$ & $\boldsymbol{n}_{\text {reactors }}$ \\
\hline 1 & 9 & 9 & 26 \\
\hline 2 & 18 & 18 & 79 \\
\hline 3 & 36 & 36 & 268 \\
\hline 4 & 40 & 40 & 317 \\
\hline 5 & 45 & 45 & 399 \\
\hline 6 & 50 & 50 & 467 \\
\hline 7 & 55 & 55 & 556 \\
\hline
\end{tabular}

The predicted NO concentration was reported in a dry basis and corrected at $3.8 \%$ volume of oxygen as reported in the experiments. The correction was performed using the following expression obtained from Turns (Turns 1996).

$$
X_{\mathrm{NO}, \mathrm{dry}-3.8 \% \mathrm{volO}_{2}}=X_{\mathrm{NO}, \mathrm{wet}} \frac{N_{\text {mix }, \text { wet }}}{N_{\text {mix }, \text { dry-3.8\%volO }}}
$$

Where $X_{N O \text {,wet }}$ is the predicted NO mole fraction in the mixture that contains water. $N_{m i x}$ are defined as follows. 


$$
\begin{aligned}
& N_{\text {mix,dry }}=4.76\left[\frac{x+\left(1-X_{O_{2, d r y}}\right) * \frac{y}{4}}{1-4.76 * X_{O_{2, d r y}}}\right]-\frac{y}{4} \\
& N_{\text {mix,wet }}=4.76\left[\frac{x+\left(1+X_{O_{2, \text { wet }}}\right) * \frac{y}{4}}{1-4.76 * X_{O_{2, \text { wet }}}}\right]+\frac{y}{4}
\end{aligned}
$$

where $x$ and $y$ are the subscripts of the hydrocarbon fuel molecule shown in Equation 3.28. $X_{O 2, d r y}$ is the dry mole fraction of the oxygen concentration $\left(X_{O 2, d r y}=0.038\right)$ and $X_{O 2, \text { wet }}$ is the oxygen concentration in the mixture that includes water.

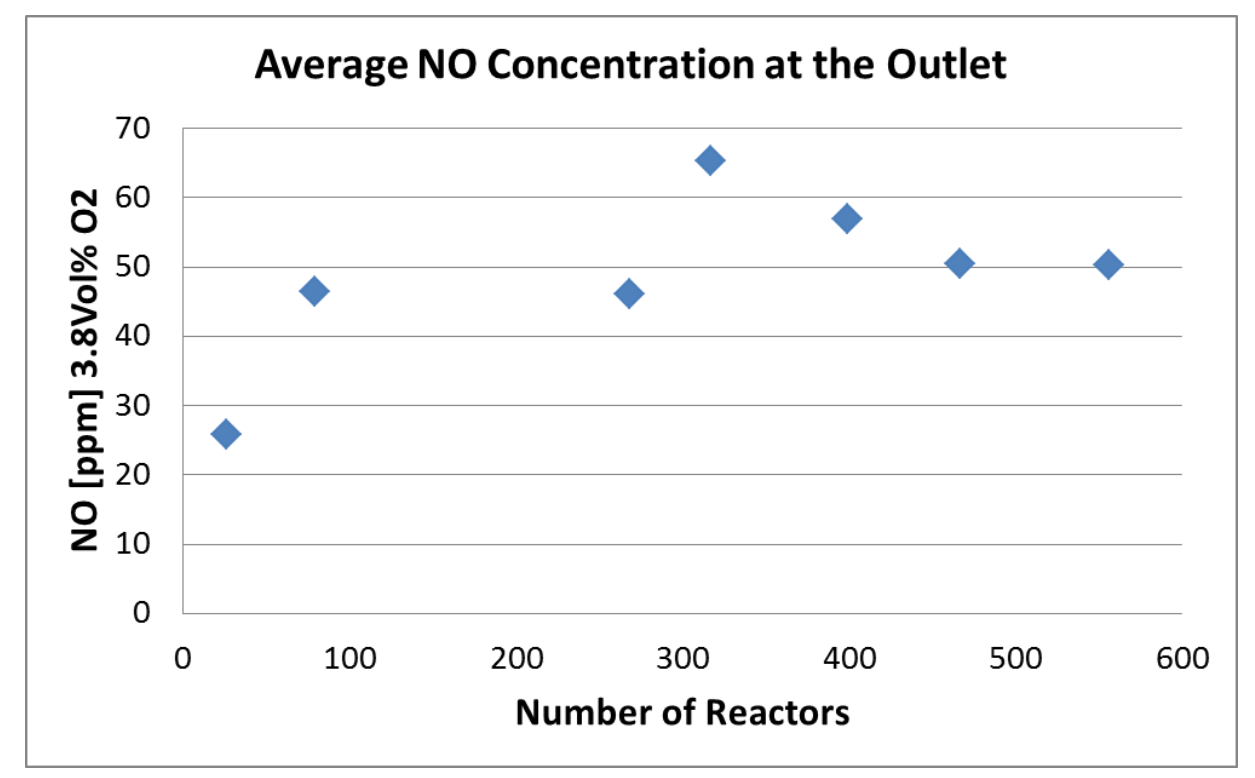

Figure 4.25. CRN Predicted NO Concentration TECFLAM Burner.

The corrected concentration in [ppm] of $\mathrm{NO}$ at the outlet of the burner as calculated from CRN is shown in Figure 4.25. The variation of the outlet concentration can be explained as follows: the calculated concentration is low with a small number of reactors because the temperature in each zone is relatively low meaning that the production of NO is less, however as the zone division became finer, there were zones with high temperatures increasing the predicted NO, but as the zone 
division became finer the size (or volume) of the reactors decreased given less time to the mixture to react. Nevertheless the predicted concentration at the outlet of the reactor is converges to circa 50 [ppm]. This concentration overpredicts the measured NO concentration $\sim 20$ [ppm]. It was expected to have an over prediction of NO because the CFD results overpredicted the temperature as shown in Figure 3.21. It has been reported that above $1800[\mathrm{~K}]$ the production of NO is mainly due to the thermal pathway (Turns 1996). The predicted CFD temperatures were approximately 250 [K] higher than the experimental values. The results of the CRN approach explored in the present study greatly depend on the CFD results. Due to the utilization of simple models in the predicted fields the results were overestimated.

A previously reported CRN study produced better agreement to the experimental NO concentration by modifying the volume of the reactors of the CRN or by defining custom average temperatures. Such techniques were not applied in this study because it was clear that the temperature was overpredicted and alterations to the reactor volume of the temperature will be fictitious. 
The CPU time needed to calculate the solution of the CRN is shown in Figure 4.26.

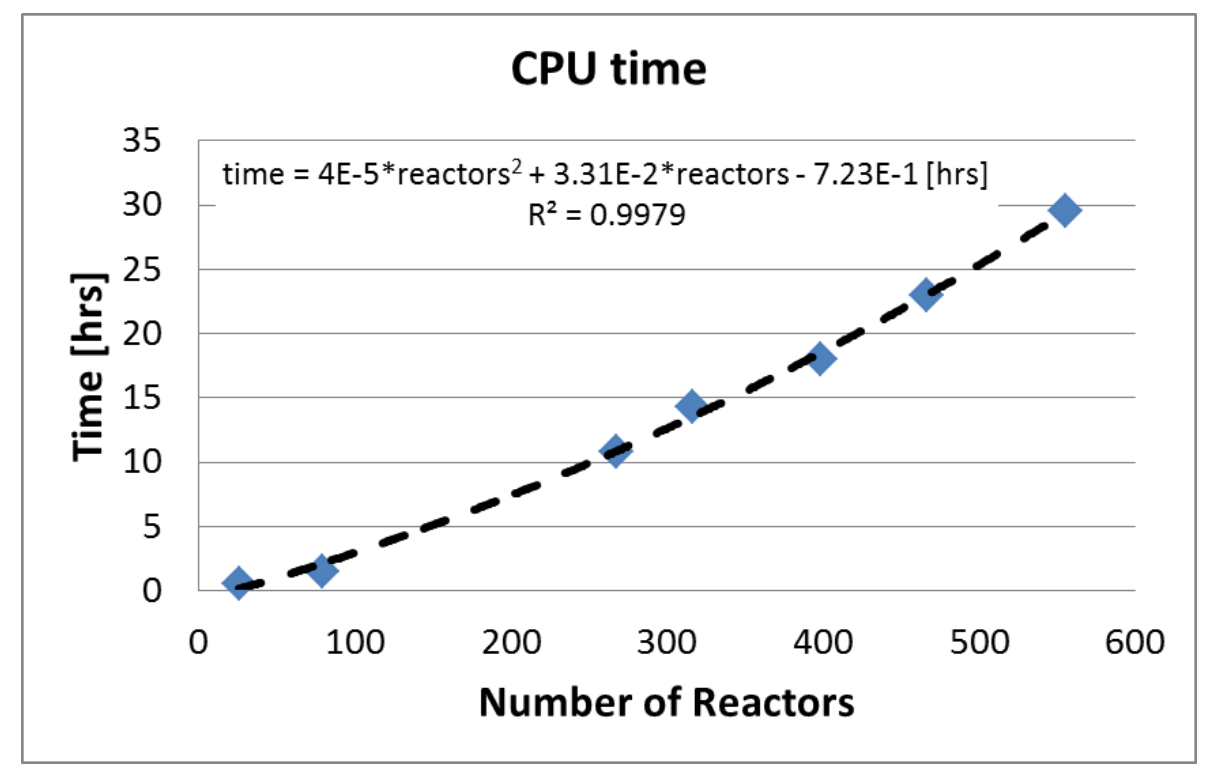

Figure 4.26. CRN CPU Time TECFLAM Burner.

The CPU time increases with the number of reactors as shown in Figure 4.26. Results from Figure 4.25 showed that the NO concentration converges to the value of 50 [ppm] which is close to the value obtained with 79 reactors. So the minimum time necessary to calculate the solution is 1.5 [hrs].

The CFD solution needed 35 [hrs] running in four processors to achieved a converged solution. The solution took this amount of time because of the solution procedure that requires to start the solution with a very high temperature inlet $\left(T_{\text {in }}=1500[\mathrm{~K}]\right)$ and then it has to be gradually decreased until it reaches the actual value $\left(T_{i n}=300[\mathrm{~K}]\right)$. The equivalent serial time needed to achieve the solution using Amdhal's law is approximately 121 [hrs]. According to the procedure used in Section 4.4.2. the total time to obtain the CRN solution is the time needed by the CFD simulation (using relatively simple models) plus the CPU time needed by the CRN, $t_{\text {total }}=122.5$ [hrs]. 
It was not possible to compare the CFD solution of this problem with the CFD results using the ARM9 reduced mechanism because of numerical issues. These issues arouse because the ARM9 was optimized to work with premixed combustion. The present simulation of the TECFLAM burner is non-premixed this means that there is a wide range of fuel-air equivalence ratios in the simulations for which the ARM9 was tuned. 


\section{Chapter 5: $\quad$ Log-Time Integration Method}

A novel integration scheme was applied to reduce the simulation time needed by the PSRs and PFRs. Solution of the PSRs and PFRs was performed using the PSR code available in CHEMKIN (Glarborg, et al. 1986). The PSR-CHEMKIN computer program predicts the species concentration using the damped Newton method, the solution method uses as initial condition the equilibrium composition at the given pressure and temperature. In order to accelerate the calculations a novel solution method was proposed, namely the Log-Time Integration Method (LTIM) (Escobar, et al. 2012). A detailed description of this method along with its applications is provided next.

\subsection{Zero Dimensional Transient Model Applications}

\subsubsection{Model Development}

A transient zero dimensional problem is used to explain the development of the LTIM. The integration method was applied to the rate equation for a chemical species given by

$$
\frac{d\left[\rho Y_{i}\right]}{d t}=M W_{i} \omega_{i}
$$

Where $Y_{i}$ is the mass fraction of the specie $i, \rho$ is the mixture density, $\omega_{i}$ is the source term (production or consumption) of the specie $i$, and $M W_{i}$ is the molecular weight of the specie $i$.

A non-dimensional time variable is defined in terms of the chemical reaction characteristic time, $t_{\text {chem }}$. 


$$
t^{*}=\frac{t}{t_{\text {chem }}}
$$

In order to use large time steps during the integration, the non-dimensional time was transformed into the logarithmic space as follows.

$$
\tau=\ln \left(\frac{\alpha t^{*}+t_{r e f}^{*}}{\alpha}\right)
$$

Substituting the logarithmic time variable into the governing Equation 5.1 yields.

$$
\frac{d\left[\rho Y_{i}\right]}{d \tau}=t_{\text {chem }}\left(\frac{\alpha t^{*}+t_{r e f}^{*}}{\alpha}\right) M W_{i} \dot{\omega}_{i}
$$

In this study it was found that $\alpha=1$ produces satisfactory results, $t_{r e f}^{*}$ must be always equal (or greater) to 1 in order to avoid singularities or negative values from Equation 5.3. Al calculations presented in reported in this study were performed with $t_{r e f}^{*}=1$.

Solution of Equation 5.4 was performed using the second order modified Euler method (Celik 2008). The method consists of evaluation of the right hand side of Equation 5.4 at the $n$ (old time step) and $n+1 / 2$ time levels. The advantage of transforming the time variable into a logarithmic space is that the rate of change of the function decreases in the logarithmic space allowing the use of larger time steps. This can be seen using the following example. 


$$
y=\exp (-2000 * t)+\exp (-2 * t)+1
$$

Equation 5.5 is plotted in Figures 5.1 a) and 5.1 b). It is seen that the transformation into the logarithmic space 5.1b) is favorable because the large slope near time $=0[\mathrm{~s}]$ was decreased when compared to the real time space 5.1 a).

a)

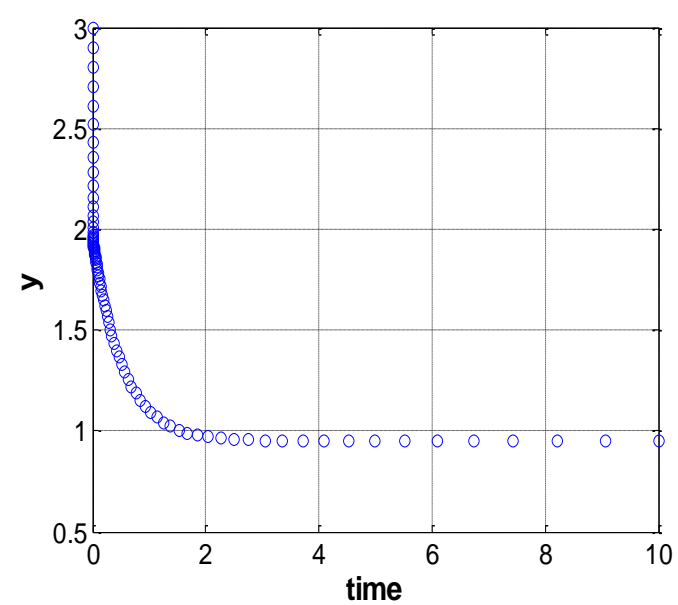

b)

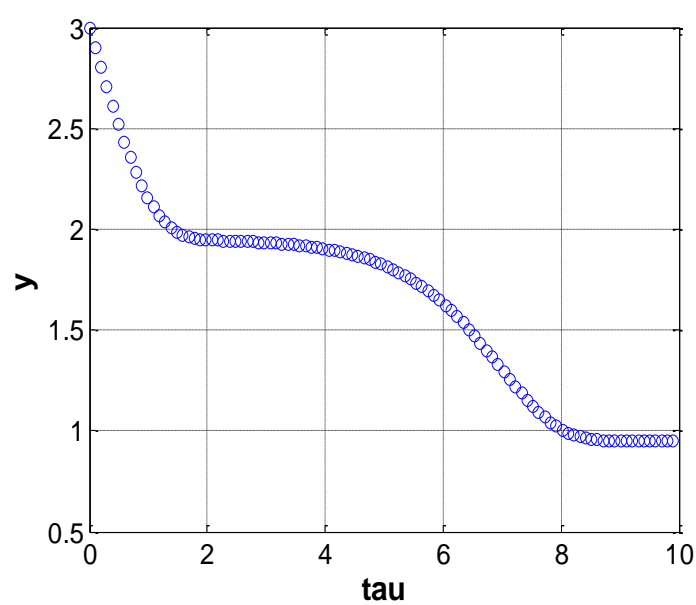

Figure 5.1. a) Equation 5.5 Variation with Time [s] , b) Equation 5.5 Variation along Logarithmic Time Space.

In order to quantify the performance of the LTIM with compared to other methods for the solution of stiff equations, the LTIM was applied to different reacting system problems which are presented in the following sections.

\subsubsection{Zero Dimensional Transient Model - NO System}

The LTIM was compared against the fifth order Runge-Kutta (R.K. $5^{\text {th }}$ order) method (Cash and Karp 1990). The comparison was done for an initial value problem. The problem consists in the prediction of the mass fraction for each of the chemical species that participate in the following reaction. 


$$
\mathrm{N}+\mathrm{O}_{2} \leftrightarrow \mathrm{NO}+\mathrm{O}
$$

The Reaction described in Equation 5.6 is one of the main reactions in the calculation of thermal NO production, the rates of consumption or production of the chemical species are given in the Arrhenius form $k=A T^{b} \exp \left(-\frac{E_{a}}{R T}\right)$. Rate coefficients values were found in Turns (Turns 1996) and are listed next; $A_{f}=1.8 \times 10^{7}\left[\mathrm{~m}^{3} / \mathrm{kmol}-\mathrm{s}-\mathrm{K}\right], b_{f}=1, \frac{E_{a, f}}{R}=4680[\mathrm{~K}]$, and $A_{r}=3.8 \times 10^{6}$ $\left[\mathrm{m}^{3} / \mathrm{kmol}-\mathrm{s}-\mathrm{K}\right], b_{r}=1, \frac{E_{a, r}}{R}=20820[\mathrm{~K}]$, where the subscripts $f$ and $r$ stand for forward and backward respectively. Reaction rate units are $\left[\mathrm{m}^{3} / \mathrm{kmol}-\mathrm{s}\right]$. The source term in Equation 5.1 is calculated in the following form.

$\dot{\omega}_{i}=\sum_{j=1}^{L} R_{i, j}$

Where $R_{i, j}$ was calculated using Equation 3.17, $j$ is the reaction index (for this case $j=1$ ) and the $L$ is the total number of reactions.

The integration of Equation 5.1 started from the following initial condition; mass fractions were set equal to 0.25 for all species, the temperature and pressure were fixed at $1000[\mathrm{~K}]$ and 1 [atm] respectively.

The solution of equation 5.1 was compared with the R.K. $5^{\text {th }}$ order integration method. The R.K. $5^{\text {th }}$ order integration method was especially designed for rapidly varying functions. The method consists of the evaluation of six slopes. Equation 5.8 shows how mass fraction at the next time step is calculated. 
$Y^{n+1}=Y^{n}+h\left[c_{1} k_{1}+c_{2} k_{2}+c_{3} k_{3}+c_{4} k_{4}+c_{5} k_{5}+c_{6} k_{6}\right]$

where $Y$ is mass fraction, $h$ is the time step, $k$ is the right hand side of Equation 5.1 evaluated at different fractional time steps, and $c$ values are constants that weight the right-hand side values calculated at different fractional time steps. The R.K. $5^{\text {th }}$ order method can be reviewed in the Appendix 2 section of the present study.

a)

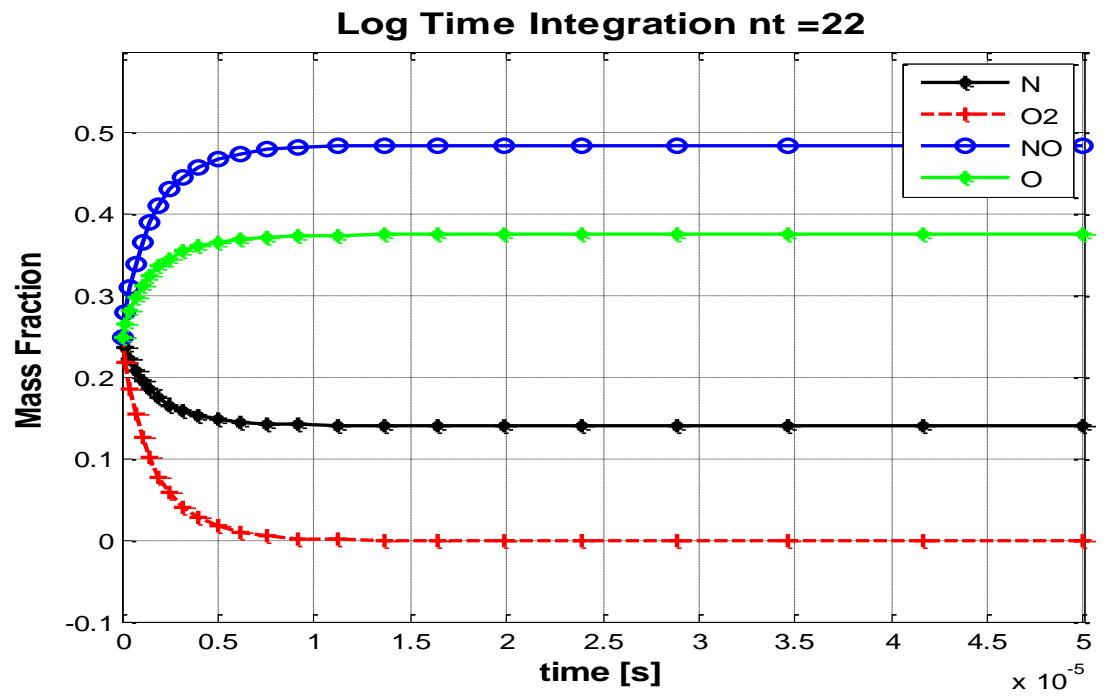

b)

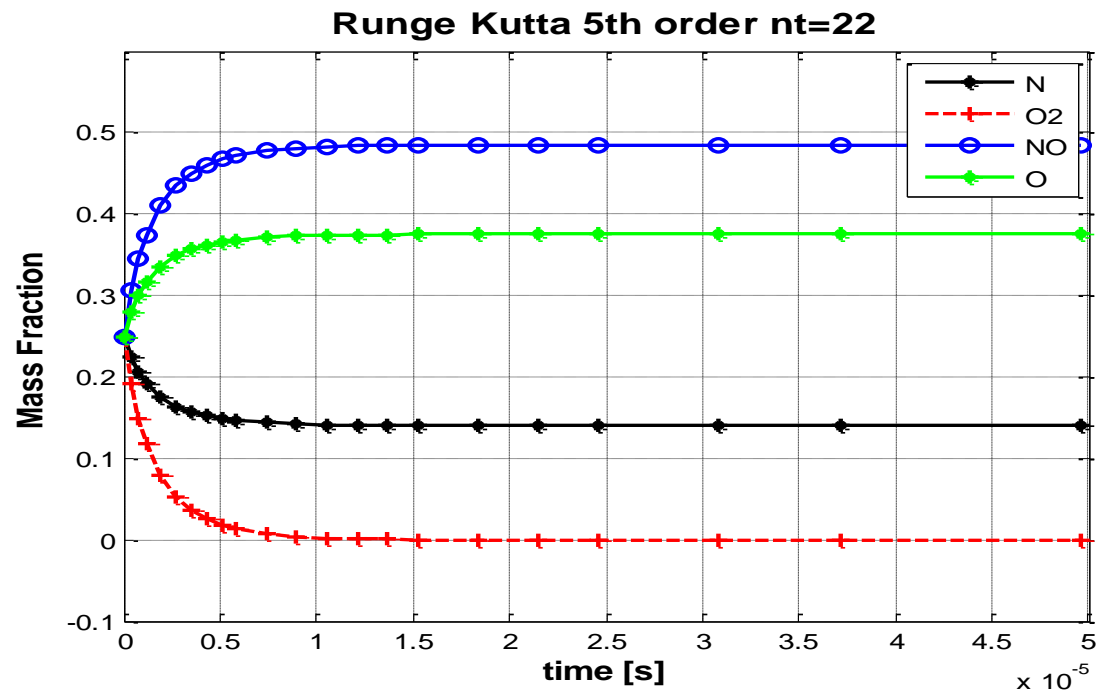

Figure 5.2. Transient Solution of Reaction 5.6. a) LTIM, b) R.K. $5^{\text {th }}$ Order Method. 
The predicted mass fractions (Figure 5.2) did not vary after $5 \times 10^{-5}[\mathrm{~s}]$. Results show that the LTIM predictions match closely the results obtained using a higher order integration method. But the LTIM needed 0.02 [s] CPU time to complete the simulation whereas the R.K. $5^{\text {th }}$ order method took 0.10 [s] CPU time. The CPU time was recorded in a PC with an Intel® Core Duo 2.4 [GHz] microprocessor with 2 [GB] of RAM. The time needed for the R.K. $5^{\text {th }}$ order method is considerably larger because it needs more calculations per time step in order to calculate the six slopes that is required per time step.

\subsubsection{Zero Dimensional Transient Model - Methane-Air System}

The LTIM was also tested for a more complex chemical mechanism and the results were also compared with the R.K. $5^{\text {th }}$ order method. Equation 5.1 was employed to solve the evolution of a methane-air system at constant temperature and pressure. The mechanism employed in these simulations was the five step ARM9 reduced mechanism originally developed by Mallampalli et al. (Mallampalli, Chen and Fletcher 1998). A mixture of methane air with an equivalence ratio equal to $0.9, T=2135[\mathrm{~K}]$, and $P=1[\mathrm{~atm}]$ was used as initial condition.

The solution of the system is carried out for a large time in order to compare the results with equilibrium values $(t \rightarrow \infty)$. Equilibrium results were obtained using the Gordon-McBride program (Chemical Equilibrium with Applications 2010).

Table 5.1. ARM9 Mass Fractions.

\begin{tabular}{|c|c|c|c|}
\hline Specie & Gordon-McBride & LTIM & R.K. $^{\text {th }}$ \\
\hline $\mathbf{H}_{\mathbf{2}}$ & $6.79 \times 10^{-5}$ & $6.79 \times 10^{-5}$ & $6.77 \times 10^{-5}$ \\
\hline $\mathbf{O}_{\mathbf{2}}$ & $2.13 \times 10^{-3}$ & $2.30 \times 10^{-2}$ & $2.30 \times 10^{-2}$ \\
\hline $\mathbf{O H}$ & $1.85 \times 10^{-3}$ & $1.72 \times 10^{-3}$ & $1.71 \times 10^{-3}$ \\
\hline $\mathbf{H}_{\mathbf{2}} \mathbf{O}$ & $1.10 \times 10^{-1}$ & $1.10 \times 10^{-1}$ & $1.10 \times 10^{-1}$ \\
\hline $\mathbf{C H}_{\mathbf{4}}$ & $1.46 \times 10^{-19}$ & $1.36 \times 10^{-19}$ & $1.36 \times 10^{-19}$ \\
\hline $\mathbf{C O}$ & $2.35 \times 10^{-3}$ & $2.37 \times 10^{-3}$ & $2.36 \times 10^{-3}$ \\
\hline $\mathbf{C O} \mathbf{2}$ & $1.33 \times 10^{-1}$ & $1.33 \times 10^{-1}$ & $1.33 \times 10^{-1}$ \\
\hline $\mathbf{N O}$ & $3.33 \times 10^{-3}$ & $3.33 \times 10^{-4}$ & $3.33 \times 10^{-4}$ \\
\hline $\mathbf{N}_{\mathbf{2}}$ & $7.27 \times 10^{-1}$ & $7.28 \times 10^{-1}$ & $7.28 \times 10^{-1}$ \\
\hline
\end{tabular}


Table 5.1 shows that LTIM predictions matched the equilibrium values calculated using the Gordon-McBride program for the species; $\mathrm{H}_{2}, \mathrm{OH}, \mathrm{H}_{2} \mathrm{O}, \mathrm{CH}_{4}, \mathrm{CO}, \mathrm{CO}_{2}, \mathrm{~N}_{2}$ (maximum difference is $7 \%$ ). LTIM predictions are close (maximum difference is $0.6 \%$ ) to the values obtained using the R.K. $5^{\text {th }}$ order method. Both approaches, LTIM and R.K. $5^{\text {th }}$ order showed a significant difference in the predicted mass fractions of $\mathrm{O}_{2}$ and $\mathrm{NO}$ (maximum difference is $98 \%$ ) when compared to the equilibrium values. This is because the NO reactions are slow and it is necessary to use a very large tie in order to allow the system to reach equilibrium. Since the difference between the LTIM and the R.K. $5^{\text {th }}$ order was very small (maximum difference is $6 \%$ ) and the difference between the predicted mass fraction of the rest of the species with the equilibrium values it was decided to stop the simulation and not reach complete equilibrium.

\subsubsection{Zero Dimensional Transient Model - Propane-Air System}

Reduced mechanisms available in the literature were created by optimizing the reaction rates to match experimental results for certain operational conditions. As a product of the optimization, the final rates have the peculiarity of having some of the species concentrations elevated to a negative power which creates a very difficult problem to handle. An example of such reduced reaction mechanisms is the propane-air mechanism developed by Kiehene et al. (Kiehne, Matthews and Wilson 1987). The propane mechanism is composed of eight reactions (Table 5.2) and eight species $\left(\mathrm{C}_{3} \mathrm{H}_{8}, \mathrm{C}_{2} \mathrm{H}_{4}, \mathrm{H}_{2}, \mathrm{O}_{2}, \mathrm{CO}, \mathrm{CO}_{2}, \mathrm{H}_{2} \mathrm{O}, \mathrm{N}_{2}\right.$ as an inert gas).

Table 5.2. Summary of the Modified Eight-Step Kinetic Model.

$$
\begin{gathered}
2 \mathrm{C}_{3} \mathrm{H}_{8} \rightarrow 3 \mathrm{C}_{2} \mathrm{H}_{4}+2 \mathrm{H}_{2} \\
\mathrm{C}_{2} \mathrm{H}_{4}+\mathrm{O}_{2} \rightarrow 2 \mathrm{H}_{2}+2 \mathrm{CO} \\
2 \mathrm{CO}+\mathrm{O}_{2} \rightarrow 2 \mathrm{CO}_{2} \\
2 \mathrm{H}_{2}+\mathrm{O}_{2} \rightarrow 2 \mathrm{H}_{2} \mathrm{O} \\
3 \mathrm{C}_{2} \mathrm{H}_{4}+2 \mathrm{H}_{2} \rightarrow 2 \mathrm{C}_{3} \mathrm{H}_{8} \\
2 \mathrm{H}_{2}+2 \mathrm{CO} \rightarrow \mathrm{C}_{2} \mathrm{H}_{4}+\mathrm{O}_{2} \\
2 \mathrm{CO}_{2} \rightarrow 2 \mathrm{CO}+\mathrm{O}_{2}
\end{gathered}
$$




$$
2 \mathrm{H}_{2} \mathrm{O} \rightarrow 2 \mathrm{H}_{2}+\mathrm{O}_{2}
$$

With rate expressions

$$
\begin{aligned}
& R_{1}=f_{1}(P) * 2.089 x 10^{17} \exp (-49600 / R T)\left[C_{3} H_{8}\right]^{0.50}\left[O_{2}\right]^{1.07}\left[C_{2} H_{4}\right]^{0.40} \\
& R_{2}=f_{2}(P) * 2 x 10^{13} \exp (-50000 / R T)\left[C_{2} H_{4}\right]^{0.90}\left[O_{2}\right]^{1.18}\left[C_{3} H_{8}\right]^{-0.37} \\
& R_{3}=S(\phi) * 1.5 x 10^{13} \exp (-40000 / R T)[C O]^{1.0}\left[O_{2}\right]^{0.25}\left[H_{2} O\right]^{0.50} \\
& R_{4}=3.311 \times 10^{13} \exp (-38100 / R T)\left[H_{2}\right]^{0.85}\left[O_{2}\right]^{1.42}\left[C_{2} H_{4}\right]^{-0.56} \\
& R_{5}=4.920 \times 10^{8} \exp (-49600 / R T)\left[C_{3} H_{8}\right]^{0.127}\left[O_{2}\right]^{1.07}\left[C_{2} H_{4}\right]^{0.40} \\
& R_{6}=2.25 \times 10^{9} \exp (-50000 / R T)\left[C_{2} H_{4}\right]^{0.528}\left[O_{2}\right]^{1.18}\left[C_{3} H_{8}\right]^{-0.37} \\
& R_{7}=4.16 x 10^{16} T^{-1 / 2} \exp (-106950 / R T)\left[C O_{2}\right]^{1.0}\left[O_{2}\right]^{-0.25}\left[H_{2} O\right]^{0.50} \\
& R_{8}=6.12 \times 10^{15} T^{-1 / 2} \exp (-100586 / R T)\left[H_{2}\right]^{-0.153}\left[O_{2}\right]^{0.916}\left[C_{2} H_{4}\right]^{-0.563}\left[H_{2} O\right]^{1.0}
\end{aligned}
$$

According to Kiehene et al. (Kiehne, Matthews and Wilson 1987) $R_{j}$ is given in [gmole/ $/ \mathrm{cm}^{3}-\mathrm{s}$ ], $T$ is in $[\mathrm{K}], R$ is the universal gas constant 1.986 [cal/K-gmole], and the specie concentration is in $\left[\mathrm{gmole} / \mathrm{cm}^{3}\right]$. The mechanism shown in Table 5.2 was developed in such a way that each reaction only proceeds in the forward direction. The optimization process results in expressions which, in some circumstances, depend on three species as shown in Table 5.2 and not only in two species as it is in the elementary reactions. The feature that makes the calculation of the composition extremely difficult is the negative exponents that appear in the rate expressions. For example, in the $R_{2}$ expression the power of $\mathrm{C}_{3} \mathrm{H}_{8}$ is negative. The propane concentration for most combustions problems tends to be very small in the regions where the combustion was completed such as post-flame regions or central recirculation zones as the ones reported in Chapter 3 of the present study. The problem arises when the concentration is close to zero, due the numerical errors 
the predicted concentration tends to go to a negative value. Negative values for species concentrations are unphysical and in most of the codes that calculate species concentration the negative concentration is eliminated and the concentration is replaced by a small value (small = $\left.1 \times 10^{-15}\right)$. This procedure does not affect the reaction rate value when its power is a positive number but if the value is negative, as it is for propane, then a sudden change to a low concentration produces non-physical concentration values.

Calculations using the propane-air mechanism showed that the system is very stiff; for this reason it was selected to test the LTIM. Calculations were performed using the LTIM procedure described in Section 5.1.1. The goal was to calculate the accuracy of the LTIM for the transient evolution of the results and to compare with the equilibrium results. LTIM results were compared with the simple but effective Euler method.

The initial condition composition was set to have an equivalence ratio equal to 0.7 , a constant temperature $=1500[\mathrm{~K}]$, and pressure fixed at $1[\mathrm{~atm}]$. No analytic transient solution is available for the current system so the predicted transient values of the composition using the explicit Euler method with a very small constant time step, $d t$, were used as the 'exact' solution. The error was calculated as the difference between the calculated and the reference solution values.

The explicit Euler method used in the calculation of the species concentration is given by

$$
Y_{i}^{n+1}=Y_{i}^{n}+d t \frac{M W_{i} \dot{\omega}_{i}}{\rho}
$$

Where $\dot{\omega}_{i}$ was evaluated using the chemical species at the previous time step $n$. In order to accelerate the calculation of the Euler method a variable time step was determined using 


$$
d t=\frac{d t_{\max }}{\log \left(\exp (1)+\max \left(\operatorname{abs}\left(\dot{\omega}_{i}\right)\right)^{n}\right)}
$$

Equation 5.10 shows how the $d t$ is adjusted depending on the value of the reaction rate. In the current approach a maximum $d t_{\max }$ is selected to avoid the time step becoming too large. The assumption for the adjustable $d t$ is if the reaction is happening too fast that means the reaction rate will become large so the $d t$ used in the calculation will be smaller. But when the system is approaching the state of equilibrium, the reaction rate will tend to zero and thus to the $d t_{\max }$ value. The extra term $\exp (1)$ was added to the expression as a safety mechanism to division by zero. Results are shown in Figure 5.3. 
a)

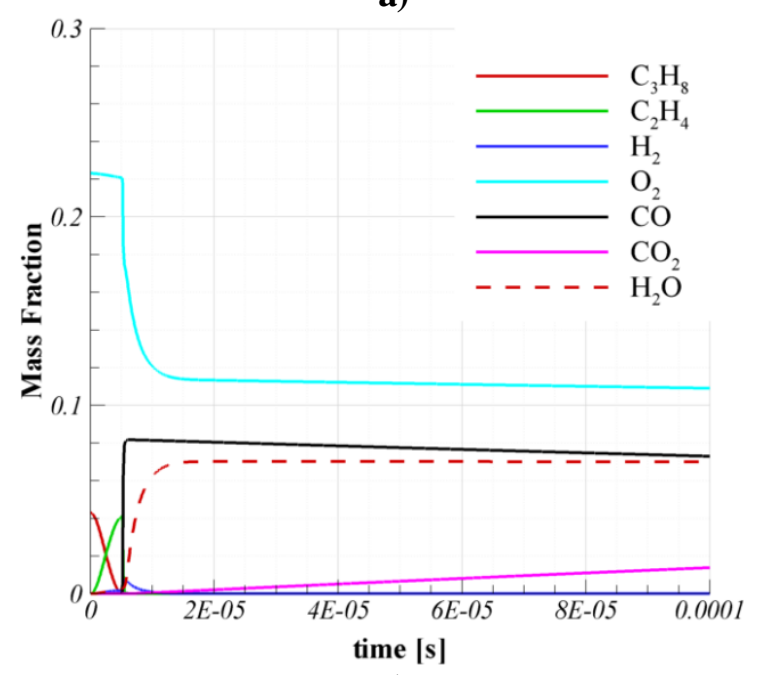

c)

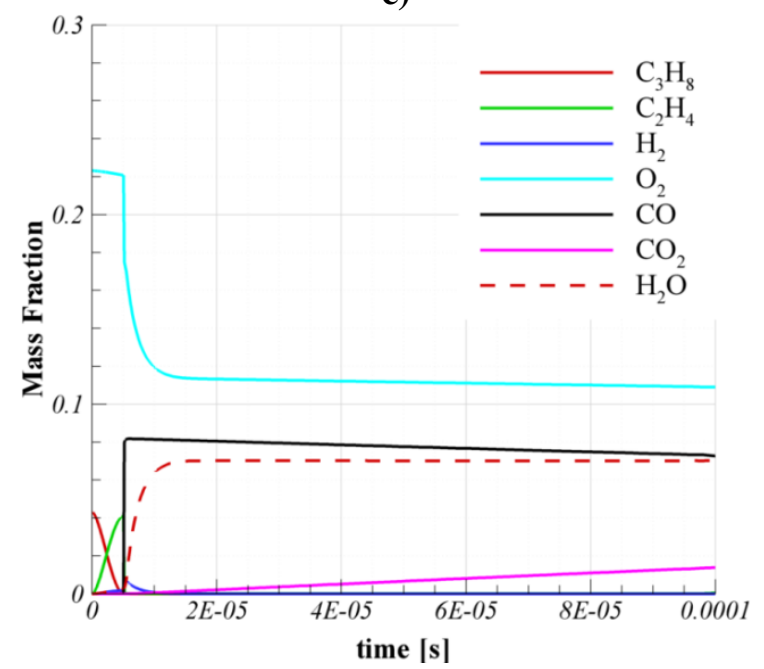

b)

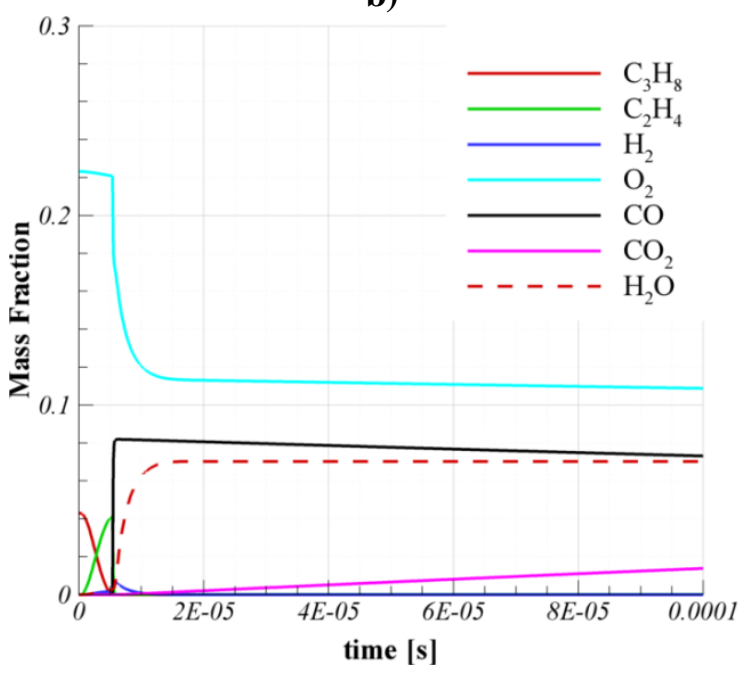

Figure 5.3. Transient Mass Fraction of Propane-Air Mechanism, $t_{\max }=1 \times 10^{-4}[\mathrm{~s}]$. a) Explicit Euler Constant $d t$, b) Explicit Euler Variable $d t$, c) LTIM.

In the calculations using the explicit Euler method the $d t$ was set to a constant value of $1 \times 10^{-7}[\mathrm{~s}]$. For the variable $d t$ case (Figure $5.3 \mathrm{~b}$ )) the best results were found for $d t_{\max }=2.1 \times 10^{-6}[\mathrm{~s}]$ and the power $n=2$ in Equation 5.10. The LTIM used the following parameters; $\alpha=1, t_{r e f}{ }^{*}=1$, $t_{\text {chem }}=1 \times 10^{-6}[\mathrm{~s}]$, and 221 time steps. Figure 5.3 shows that for all cases the transient solution is similar. In order to assess the accuracy of each method another case with the explicit Euler method using a $d t=1 \times 10^{-9}[\mathrm{~s}]$ was used as the reference 'exact' solution. 
The $L 2_{\text {norm }}$ of the error (Equation 5.11) is used as a metric for accuracy check.

$$
L 2_{n o r m}=\frac{s q r t\left(\sum_{j=1}^{s p e} \sum_{i=1}^{n t}\left(Y_{r e f_{-} j, i}-Y_{\text {pred }_{-}, i, i}\right)^{2}\right)}{s p e^{*} n t}
$$

Here spe is the total number of species, $n t$ is the total number of time steps, $Y_{\text {ref }}$ is the mass fraction from the reference case, and $Y_{\text {pred }}$ is the predicted mass fraction using any of the three aforementioned methods.

Table 5.3. Propane Air Mechanism Summary.

\begin{tabular}{|c|c|c|c|}
\hline Method & Time steps & CPU time [s] & L2 $_{\text {norm }}$ \\
\hline $\begin{array}{c}\text { Explicit Euler } \\
\boldsymbol{d} \boldsymbol{t}=\text { const }\end{array}$ & 1000 & 0.3330 & $\mathbf{7 . 5 6 6 1 \times 1 0 ^ { - 6 }}$ \\
\hline $\begin{array}{c}\text { Explicit Euler } \\
\boldsymbol{d} \boldsymbol{t}=\text { var }\end{array}$ & 369 & $\mathbf{0 . 0 9 8 6}$ & $4.9276 \times 10^{-4}$ \\
\hline LTIM & $\mathbf{2 2 1}$ & 0.1170 & $4.2110 \times 10^{-5}$ \\
\hline
\end{tabular}

Table 5.3 shows that the method that takes the least number of time steps to reach maximum time step is the LTIM, the fastest method is the Euler method with variable time step because it only requires the evaluation of one slope per time step and the LTIM requires two, and the smallest $L 2_{\text {norm }}$ was obtained with the Explicit Euler with constant $d t$. Since the error is a function of the size of the time steps it is expected than the case with the smallest $d t$ has the least error.

Table 5.3 also shows that the utilization of a variable $d t$ depending on the magnitude of the variation of the right hand side of the solved equation helps to increase the speed of the solution. This feature will be used in the following section when the LTIM is applied in the solution of a perfectly stirred reactor (PSR). 


\subsection{PSR Test Case}

The main component of the CRN simulations is the perfectly stirred reactor (PSR). The current study proposes a novel method to solve this ideal reactor with enough speed that could be comparable with the available commercial codes such as the PSR (Glarborg, et al. 1986) which is part of the CHEMKIN software.

\subsubsection{PSR-CHEMKIN}

The perfectly stirred reactor is an ideal reactor in which perfect mixing is achieved inside the control volume. Figure 4.7 shows a diagram of a PSR in which the mass $\dot{m}$ enters the domain with an inlet composition and temperature. The mixture inside the reactor changes due to the chemical reactions that take place. Due to the assumption of perfect mixing inside the reactor the composition of the mixture is same anywhere inside the reactor so the spatial dependence of the mixture composition disappears producing a zero dimensional transient mass conservation equation:

$\frac{d m_{i, c v}}{d t}=\dot{m}_{i}^{\prime \prime \prime} V+\dot{m}_{i, \text { in }}-\dot{m}_{i, o u t}$

The left hand side of Equation 5.12 represents the rate at which the mass of specie $i$ accumulates inside the control volume, the first term on the right hand side is related to the mass consumption or production of the specie $i$ inside the control volume; the second term is the mass flow of the specie $i$ into the control volume, and the last term is the mass flow of the specie $i$ leaving the control volume. When Equation 5.12 is applied to the PSR assuming steady state and no diffusion fluxes at the inlet and the outlet boundaries of the reactor so that $\dot{m}_{i}=\dot{m} Y_{i}$, Equation 5.12 reduces to 
$\dot{\omega}_{i} M W_{i} V+\dot{m}\left(Y_{i, \text { in }}-Y_{i, \text { out }}\right)=0$

Equation 5.13 is valid for all the chemical species inside the reactor. The temperature was assumed constant inside the PSR used for the CRN calculations for this reason it is not required to solve the energy equation for the current problem.

The PSR-CHEMKIN program solves Equation 5.13 using the modified damped Newton method. The Newton's method is an iterative solution procedure, it substitutes arbitrary values of the vector $\phi$ (for the current problem $\phi=Y_{i}$ ) into the governing equations. The method iterates until it finds a set of values of the vector $\phi$ that will generate a residual vector of the governing equations $F$ equal to zero. The goal is to find $\phi$ such that.

$F(\phi)=0$

The modified damped Newton method requires that the initial estimate of $\phi$ must be a value close to the solution of the system otherwise the method will diverge. The PSR-CHEMKIN uses the equilibrium concentrations at the given temperature and pressure as the initial guess. With the initial estimate the Newton algorithm starts to iterate until the solution converges. The new iteration value of the variable $\phi$ is calculated by the Newton method using the following expression.

$\phi^{(n+1)}=\phi^{(n)}-\lambda^{(n)}\left(J^{(n)}\right)^{-1} F\left(\phi^{n}\right)$

Where the Jacobian matrix, $\boldsymbol{J}$, is equal to $\partial F / \partial \phi_{k}$, and $\lambda^{(n)}$ is a damping factor. Evaluation of the Jacobian in Equation 5.15 makes the calculations extremely costly for this reason the PSR- 
CHEMKIN does not evaluate the Jacobian every iteration but it saves the Jacobian for several iterations. The value of $\lambda$ is restricted to be between 0 and 1 ; the calculation of this variable depends on several criteria but it is designed to keep the iteration stable and within bounds. A detailed description of the solution algorithm, called TWOPNT, can be found in (Grcar 1992). The solution is considered converged when the following condition is satisfied.

$|\Delta \phi| \leq \max (A, R *|\phi|)$

Where $A$ is the absolute tolerance and $R$ is the relative tolerance.

\subsubsection{PSR-LTIM}

The LTIM was applied to the solution of a PSR. The LTIM works differently than the modified Newton's damped method because it is not an iterative method. The governing equation solved by the LTIM is Equation 5.17. Integration of Equation 5.17 was performed until the term on the left hand side of the equation approaches zero, as $t \rightarrow \infty$.

$$
\frac{d\left(\rho V Y_{i, c v}\right)}{d t}=M W_{i} \dot{\omega}_{i} V+\dot{m}\left(Y_{i, \text { in }}-Y_{i, \text { out }}\right)
$$

During the integration process of Equation 5.17 the value of the mass fraction at the old time level $Y_{i, c v}^{n}$ was the same as the $Y_{i, o u t}$ due to the assumption of perfect mixing in the reactor meaning that the concentration is same inside the reactor as what it is at its outlet. The time variable transformation described in Section 5.1.1 was applied to Equation 5.17 giving the following expression. 
$\frac{\rho V *\left(Y_{i}^{(n+1)}-Y_{i}^{(n)}\right)}{\Delta \tau}=t_{c h e m}\left(\frac{\alpha t^{*}+t_{r e f}^{*}}{\alpha}\right) *\left(M W_{i} \dot{\omega}_{i} V+\dot{m} *\left(Y_{i, i n}-Y_{i}^{(n)}\right)\right)$

where $\alpha=1$, and $t_{r e f}^{*}=1$ for the current calculations, and $t_{c h e m}=5 \times 10^{-9}[\mathrm{~s}]$.

As discussed in the propane-air section, implementation of variable time step as a function of the rate of the reactions proved to be an effective strategy that reduced the computational time. The adaptive time step strategy was implemented to the LTIM integration method as follows:

First Equation 5.18 is written as follows.

$Y_{i}^{(n+1)}=Y_{i}^{(n)}+d \tau * R H S$

where all the terms shown in the right hand side of Equation 5.18 were collapsed into the $R H S$ variable. Equation 5.19 shows that the mass fraction at the next time is equal to its old value plus the rate of change, the second term in the right hand side of Equation 5.19 can be expressed as $Y_{i}^{v a r}=d \tau * R H S$. It was found that if $Y_{i}^{v a r}$ was limited to change only a fraction $\left(\Delta Y_{i, \max }\right)$ of the previous time step, the system remained stable and predicted relatively accurate results. In order to satisfy the fractional change it is necessary to adjust the $d \tau$ accordingly using the following expression.

$d \tau=\frac{1}{\Delta Y_{i, \max }} \max \left(\frac{Y_{i}^{(n)}}{\operatorname{abs}(R H S)}\right)$

Since the mass fraction variable is an array whose size depends on the total number of chemical species it was necessary to calculate the maximum variation of the array and adjust $d \tau$ 
accordingly. In order to maintain the stability of the solution near the steady state, it was also necessary to limit the maximum $d \tau$ value. In this study it was found that a $d t_{\max }=1 \times 10^{-4}[\mathrm{~s}]$ avoided the prediction of non-physical values (the maximum $d t$ was given in real time but it was adjusted to logarithmic time inside the program).

\subsubsection{Results for PSR Simulation}

The solution of the PSR calculated using the detailed chemical mechanism GRI 3.0 for methane-air combustion had the following conditions; $T=1800[\mathrm{~K}], P=1[\mathrm{~atm}], V=5.0\left[\mathrm{~cm}^{3}\right]$, $\dot{m}_{\text {in }}=1.0[\mathrm{~g} / \mathrm{s}]$, and the initial composition is given in Table 5.4. Solution of the problem was carried out by integrating Equation 5.18 until steady state was reached.

Table 5.4. PSR Conditions.

\begin{tabular}{|c|c|}
\hline Temperature $[\mathrm{K}]$ & 1800 \\
\hline Pressure $[\mathrm{atm}]$ & 1.0 \\
\hline Volume $\left[\mathrm{cm}^{3}\right]$ & 5.0 \\
\hline Mass Flux [g/s] & 1.0 \\
\hline Inlet Oxygen Mole Fraction & 0.158872 \\
\hline Inlet Methane Mole Fraction & 0.078651 \\
\hline Inlet Carbon Dioxide Mole Fraction & 0.01938 \\
\hline Inlet Nitrogen Mole Fraction & Balance \\
\hline
\end{tabular}

Two solution procedures were used; first the solution was calculated using the inlet mixture composition as the initial composition of the reactor, the second approach used the equilibrium composition of the mixture at the given temperature and pressure of the reactor as initial condition.

It was found that using the inlet composition as the initial composition restricted the size of the time step. When calculating the solution the $\Delta Y_{i, \max }$ had to be limited to $5 \%$. But when the equilibrium composition was used as the initial condition the $\Delta Y_{i, \max }$ was limited to $50 \%$ and the results were within reasonable accuracy (the maximum difference was 1\%) for the species of interest. 
Figure 5.4 shows the transient behavior of two monitor species, namely carbon monoxide and nitrogen oxide.

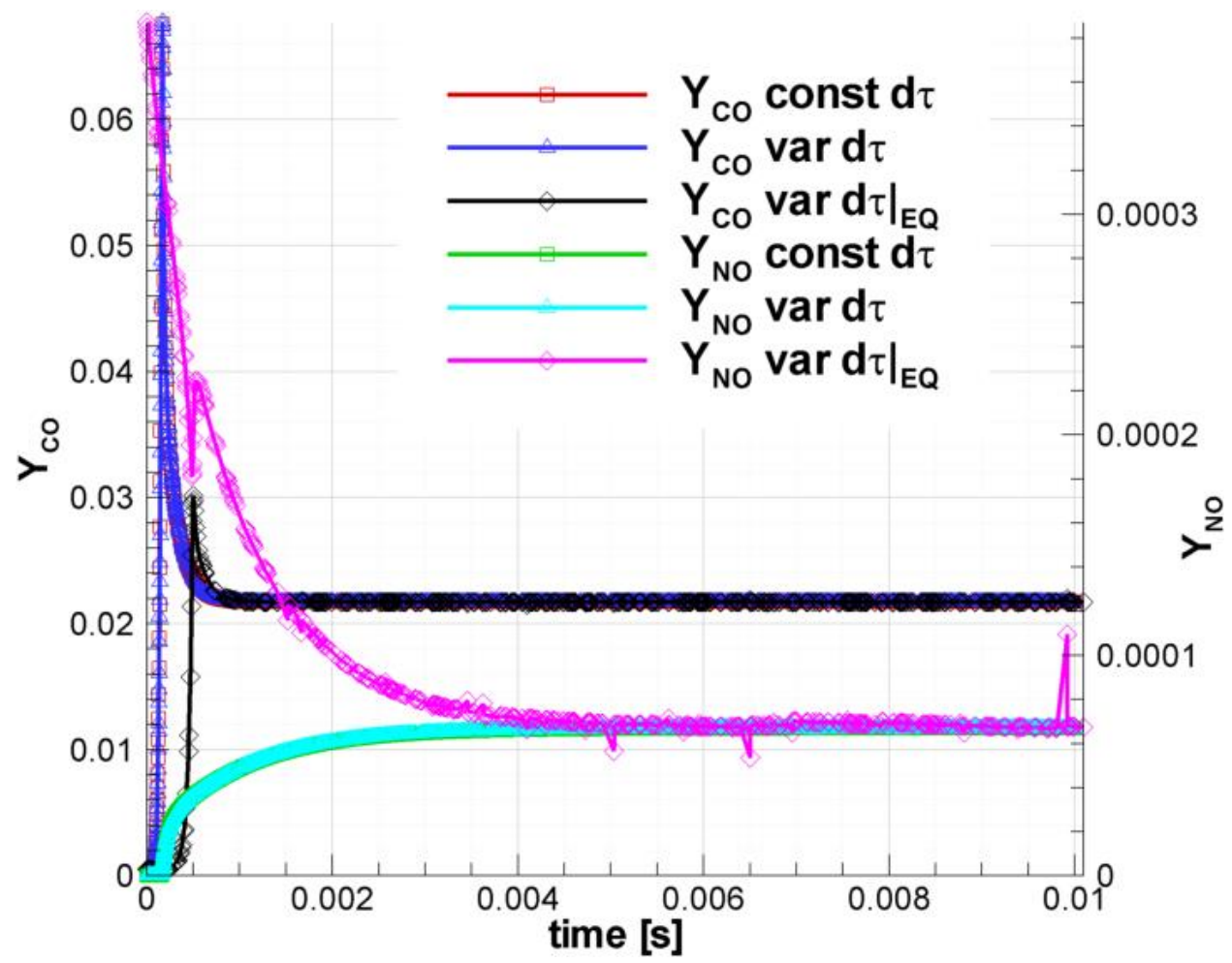

Figure 5.4. Transients of Carbon Monoxide and Nitrogen Oxide Mass Fractions from the PSRLTIM Calculations.

In Figure 5.4 three sets of calculations are depicted: (i) with constant $d \tau$ in the logarithmic space, (ii) with variable $d \tau$ in logarithmic space, and the initial composition equal to the inlet composition, and finally (iii) with variable $d \tau$ in the logarithmic space using the equilibrium composition as the initial condition. Species mass fraction reached a constant value around $t_{\max }=$ 0.01 [s]. Results obtained from the variable $d \tau$ using the equilibrium composition as the initial composition showed fluctuations which are higher than the other set of results, nevertheless the predicted values approached to the same final value reported in the other approaches. Fluctuations are due the variable $d \tau$ used for that specific time step, using large time steps increase the numerical error in the predictions. 
Utilization of the equilibrium composition partially removed the stiffness of the problem allowing the utilization of relatively large time steps. The percentage variation reported in Table 5.5 was calculated using the following expression.

$\%$ diff $=\frac{a b s\left(X_{i, \text { Chemkin }}-X_{i, L T I M}\right)}{X_{i, \text { Chemkin }}} * 100$

Table 5.5. PSR-LTIM Predicted Mole Fractions of the Species of Interest.

\begin{tabular}{|c|c|c|c|c|c|c|}
\hline & Specie & CHEMKIN & $\begin{array}{c}\text { LTIM Var } \\
\quad d \tau \\
\end{array}$ & $\begin{array}{c}\text { LTIM Var } \\
d \tau+\text { EQ }\end{array}$ & $\begin{array}{c}\text { \% diff Var } \\
d \tau\end{array}$ & $\begin{array}{c}\% \text { diff Var } \\
d \tau+\mathrm{EQ}\end{array}$ \\
\hline 1 & $\mathbf{H}_{2}$ & $8.76 \times 10^{-3}$ & $8.76 \times 10^{-3}$ & $8.75 \times 10^{-3}$ & $4.57 \times 10^{-3}$ & $8.90 \times 10^{-2}$ \\
\hline 2 & $\mathbf{O}_{2}$ & $1.61 \times 10^{-2}$ & $1.61 \times 10^{-2}$ & $1.61 \times 10^{-2}$ & $2.24 \times 10^{-1}$ & $1.99 \times 10^{-1}$ \\
\hline 3 & OH & $4.48 \times 10^{-3}$ & $4.48 \times 10^{-3}$ & $4.48 \times 10^{-3}$ & $6.70 \times 10^{-2}$ & $8.26 \times 10^{-2}$ \\
\hline 4 & $\mathrm{H}_{2} \mathrm{O}$ & $1.41 \times 10^{-1}$ & $1.41 \times 10^{-1}$ & $1.41 \times 10^{-1}$ & $2.84 \times 10^{-1}$ & $2.77 \times 10^{-1}$ \\
\hline 5 & $\mathrm{CH}_{4}$ & $1.99 \times 10^{-4}$ & $1.99 \times 10^{-4}$ & $1.99 \times 10^{-4}$ & $2.01 \times 10^{-1}$ & $1.06 \times 10^{-1}$ \\
\hline 6 & $\mathrm{CO}$ & $2.13 \times 10^{-2}$ & $2.13 \times 10^{-2}$ & $2.13 \times 10^{-2}$ & $1.69 \times 10^{-1}$ & $1.13 \times 10^{-1}$ \\
\hline 7 & $\mathrm{CO}_{2}$ & $7.45 \times 10^{-2}$ & $7.45 \times 10^{-2}$ & $7.45 \times 10^{-2}$ & $1.21 \times 10^{-2}$ & $1.21 \times 10^{-2}$ \\
\hline 8 & NO & $6.12 \times 10^{-5}$ & $6.12 \times 10^{-5}$ & $6.18 \times 10^{-5}$ & $8.01 \times 10^{-2}$ & 1.02 \\
\hline 9 & $\mathbf{N}_{2}$ & $7.28 \times 10^{-1}$ & $7.28 \times 10^{-1}$ & $7.28 \times 10^{-1}$ & $2.20 \times 10^{-2}$ & $1.65 \times 10^{-2}$ \\
\hline \multicolumn{5}{|c|}{ Maximum Variation \% } & $2.84 \times 10^{-1}$ & 1.02 \\
\hline
\end{tabular}

The results reported in Table 5.5 showed that the maximum variation for the PSR-LTIM using the inlet composition as the initial composition is about $0.28 \%$ and for the case with the equilibrium composition as the initial composition it is $1.02 \%$. The rest of the species concentration values are reported in Table 5.6.

Table 5.6. PSR-LTIM Predicted Mole Fractions Continued.

\begin{tabular}{|c|c|c|c|c|}
\hline & Specie & CHEMKIN & LTIM Var $d \tau$ & $\begin{array}{c}\text { LTIM Var } d \tau+ \\
\text { EQ }\end{array}$ \\
\hline 10 & $\mathbf{H}$ & $4.07 \times 10^{-3}$ & $4.13 \times 10^{-3}$ & $4.02 \times 10^{-3}$ \\
\hline 11 & $\mathbf{O}$ & $1.75 \times 10^{-3}$ & $1.74 \times 10^{-3}$ & $1.75 \times 10^{-3}$ \\
\hline 12 & $\mathrm{HO}_{2}$ & $6.31 \times 10^{-6}$ & $6.38 \times 10^{-6}$ & $6.24 \times 10^{-6}$ \\
\hline 13 & $\mathrm{H}_{2} \mathrm{O}_{2}$ & $3.05 \times 10^{-7}$ & $3.05 \times 10^{-7}$ & $3.08 \times 10^{-7}$ \\
\hline 14 & C & $4.43 \times 10^{-7}$ & $4.19 \times 10^{-7}$ & $4.51 \times 10^{-7}$ \\
\hline 15 & $\mathbf{C H}$ & $6.79 \times 10^{-7}$ & $1.37 \times 10^{-6}$ & $3.77 \times 10^{-7}$ \\
\hline 16 & $\mathrm{CH}_{2}$ & $3.08 \times 10^{-6}$ & $1.00 \times 10^{-20}$ & $4.06 \times 10^{-6}$ \\
\hline 17 & $\mathrm{CH}_{2}(\mathrm{~S})$ & $3.15 \times 10^{-7}$ & $2.80 \times 10^{-5}$ & $1.00 \times 10^{-20}$ \\
\hline 18 & $\mathrm{CH}_{3}$ & $5.19 \times 10^{-5}$ & $4.58 \times 10^{-5}$ & $5.21 \times 10^{-5}$ \\
\hline 19 & HCO & $3.03 \times 10^{-6}$ & $3.07 \times 10^{-6}$ & $2.85 \times 10^{-6}$ \\
\hline 20 & $\mathrm{CH}_{2} \mathrm{O}$ & $2.13 \times 10^{-5}$ & $1.98 \times 10^{-5}$ & $2.13 \times 10^{-5}$ \\
\hline 21 & $\mathrm{CH}_{2} \mathrm{OH}$ & $4.36 \times 10^{-7}$ & $1.83 \times 10^{-7}$ & $4.49 \times 10^{-7}$ \\
\hline
\end{tabular}




\begin{tabular}{|c|c|c|c|c|}
\hline 22 & $\mathrm{CH}_{3} \mathrm{O}$ & $2.92 \times 10^{-8}$ & $2.28 \times 10^{-8}$ & $2.88 \times 10^{-8}$ \\
\hline 23 & $\mathrm{CH}_{3} \mathrm{OH}$ & $6.39 \times 10^{-7}$ & $5.06 \times 10^{-7}$ & $6.43 \times 10^{-7}$ \\
\hline 24 & $\mathrm{C}_{2} \mathrm{H}$ & $6.25 \times 10^{-10}$ & $4.10 \times 10^{-10}$ & $7.86 \times 10^{-10}$ \\
\hline 25 & $\mathrm{C}_{2} \mathrm{H}_{2}$ & $1.63 \times 10^{-7}$ & $1.64 \times 10^{-7}$ & $2.08 \times 10^{-7}$ \\
\hline 26 & $\mathrm{C}_{2} \mathrm{H}_{3}$ & $1.76 \times 10^{-8}$ & $1.75 \times 10^{-8}$ & $1.79 \times 10^{-8}$ \\
\hline 27 & $\mathrm{C}_{2} \mathrm{H}_{4}$ & $1.92 \times 10^{-7}$ & $1.93 \times 10^{-7}$ & $1.97 \times 10^{-7}$ \\
\hline 28 & $\mathrm{C}_{2} \mathrm{H}_{5}$ & $3.52 \times 10^{-9}$ & $3.56 \times 10^{-9}$ & $3.63 \times 10^{-9}$ \\
\hline 29 & $\mathrm{C}_{2} \mathrm{H}_{6}$ & $1.19 \times 10^{-8}$ & $1.19 \times 10^{-8}$ & $1.20 \times 10^{-8}$ \\
\hline 30 & HCCO & $2.34 \times 10^{-8}$ & $2.00 \times 10^{-8}$ & $2.62 \times 10^{-8}$ \\
\hline 31 & $\mathrm{CH}_{2} \mathrm{CO}$ & $1.31 \times 10^{-7}$ & $1.30 \times 10^{-7}$ & $1.29 \times 10^{-7}$ \\
\hline 32 & НССОH & $9.12 \times 10^{-10}$ & $9.15 \times 10^{-10}$ & $5.87 \times 10^{-10}$ \\
\hline 33 & $\mathbf{N}$ & $5.25 \times 10^{-8}$ & $5.20 \times 10^{-8}$ & $5.33 \times 10^{-8}$ \\
\hline 34 & NH & $1.90 \times 10^{-8}$ & $1.12 \times 10^{-7}$ & $1.00 \times 10^{-20}$ \\
\hline 35 & $\mathbf{N H}_{2}$ & $1.26 \times 10^{-8}$ & $1.26 \times 10^{-8}$ & $1.26 \times 10^{-8}$ \\
\hline 36 & $\mathrm{NH}_{3}$ & $9.81 \times 10^{-9}$ & $9.81 \times 10^{-9}$ & $9.88 \times 10^{-9}$ \\
\hline 37 & NNH & $6.12 \times 10^{-9}$ & $1.00 \times 10^{-20}$ & $6.52 \times 10^{-5}$ \\
\hline 38 & $\mathrm{NO}_{2}$ & $9.22 \times 10^{-9}$ & $9.22 \times 10^{-9}$ & $9.34 \times 10^{-9}$ \\
\hline 39 & $\mathrm{~N}_{2} \mathrm{O}$ & $1.04 \times 10^{-7}$ & $1.04 \times 10^{-7}$ & $1.04 \times 10^{-7}$ \\
\hline 40 & HNO & $1.06 \times 10^{-8}$ & $1.02 \times 10^{-8}$ & $1.00 \times 10^{-20}$ \\
\hline 41 & CN & $3.72 \times 10^{-9}$ & $3.64 \times 10^{-9}$ & $3.92 \times 10^{-9}$ \\
\hline 42 & HCN & $1.13 \times 10^{-6}$ & $1.13 \times 10^{-6}$ & $1.13 \times 10^{-6}$ \\
\hline 43 & $\mathrm{H}_{2} \mathrm{CN}$ & $7.17 \times 10^{-11}$ & $8.54 \times 10^{-11}$ & $1.00 \times 10^{-20}$ \\
\hline 44 & HCNN & $1.02 \times 10^{-9}$ & $7.74 \times 10^{-10}$ & $1.26 \times 10^{-9}$ \\
\hline 45 & HCNO & $1.71 \times 10^{-8}$ & $1.71 \times 10^{-8}$ & $1.74 \times 10^{-8}$ \\
\hline 46 & HOCN & $2.27 \times 10^{-9}$ & $2.27 \times 10^{-9}$ & $2.28 \times 10^{-9}$ \\
\hline 47 & HNCO & $1.78 \times 10^{-7}$ & $1.78 \times 10^{-7}$ & $1.79 \times 10^{-7}$ \\
\hline 48 & NCO & $2.07 \times 10^{-8}$ & $2.07 \times 10^{-8}$ & $2.07 \times 10^{-8}$ \\
\hline 49 & Ar & $1.18 \times 10^{-20}$ & $9.80 \times 10^{-21}$ & $9.80 \times 10^{-21}$ \\
\hline 50 & $\mathrm{C}_{3} \mathbf{H}_{7}$ & $8.00 \times 10^{-14}$ & $7.99 \times 10^{-14}$ & $7.80 \times 10^{-14}$ \\
\hline 51 & $\mathrm{C}_{3} \mathbf{H}_{8}$ & $3.76 \times 10^{-13}$ & $3.76 \times 10^{-13}$ & $3.76 \times 10^{-13}$ \\
\hline 52 & $\mathrm{CH}_{2} \mathrm{CHO}$ & $8.60 \times 10^{-10}$ & $2.67 \times 10^{-9}$ & $8.59 \times 10^{-10}$ \\
\hline 53 & $\mathrm{CH}_{3} \mathrm{CHO}$ & $7.38 \times 10^{-9}$ & $7.38 \times 10^{-9}$ & $7.33 \times 10^{-9}$ \\
\hline
\end{tabular}

The PSR-LTIM mole fraction predictions for the rest of the chemical species of the GRI

3.0 detailed mechanism are shown in Table 5.6. The largest difference is seen for the species whose concentration became negative but PSR-LTIM code changed the value to a small value $\left(1 \times 10^{-20}\right)$.

Numerical simulations were performed using an Intel Xeon processor operating at 2.33 [GHz]. PSR-CHEMKIN and PSR-LTIM calculations were performed using serial computer programs. 


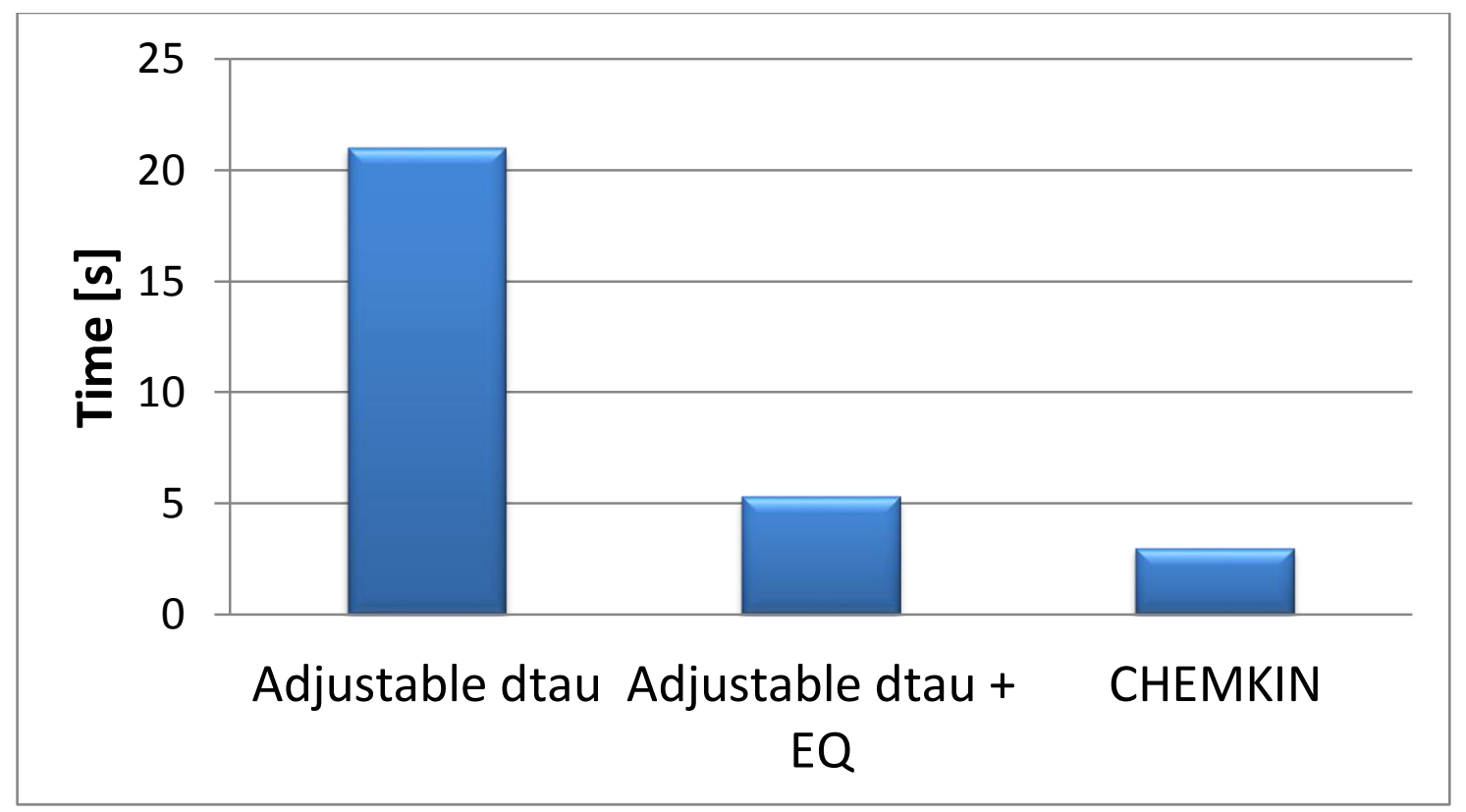

Figure 5.5. Calculated CPU Time of the PSR Calculations.

Figure 5.5 shows the calculated CPU time that took to calculate the solution of the PSR. The PSR-LTIM with variable time step using the inlet concentration as the initial composition took the longest time $(21[\mathrm{~s}])$ because the calculation used small time steps due to the stiffness of the problem. But the simulation of the PSR-LTIM using the equilibrium concentration as the initial condition allowed the code to utilize larger time steps and the time required to calculate the solution was reduced to 5.3 [s]. PSR CHEMKIN took 3.0 [s] in total. Here it should be mention that the CHEMKIN calculation also uses the equilibrium composition as the initial guess for its iterative procedure and the $3.0[\mathrm{~s}]$ of CPU time includes all the calculations (equilibrium calculations + solution of the damped Newton method).

The equilibrium composition used in the PSR-LTIM approach was calculated using an open source software called CANTERA (Goodwin, et al. 2009). The CPU time it took CANTERA to calculate the equilibrium composition was 0.7 [s]. When this time is added to the PSR-LTIM approach the total time adds up to 6 [s] compared to the 3 [s] it takes to CHEMKIN to retrieve the solution. 
It is considered that the PSR-LTIM is a reasonable fast approach to solve systems of stiff equations seen in reactive systems. The PSR-LTIM could still be optimized by reducing the time the code takes to call the subroutines related to the calculation of the chemical reaction source term (consumption or production of chemical species). The computational time could also be reduced by using polynomials instead of calculating logarithms and exponentials in the code because the computation of the aforementioned functions is computationally expensive. Another component that can be optimized is the calculation of the equilibrium composition using CANTERA. The CPU time reported here was calculated in Pentium Core Duo Machine operating at $2.4[\mathrm{GHz}]$. Current equilibrium calculations were performed in the CANTERA version that is linked to MATLAB. MATLAB programs run significantly slower than similar versions written in FORTRAN or C++ languages. There is a CANTERA version in C++ so it is believed that the CPU time of the equilibrium calculations will be reduced if this version is used.

\subsubsection{Thin Shear Layer Reactor}

In the first stage of the present study it was envisioned to further reduce the computational time of the CRN by the utilization of a novel reactor which was called Thin Shear Layer Reactor (TSLR). It was proposed to use this reactor in the combustor region that cannot be accurately represented by PSRs or PFRs because the flow was basically one dimensional but the temperature varied perpendicular to the flow direction. This zone is usually located in the flame region (zone A Figure 3.2), this zone using CRN is modelled by a series of PSRs. It was intended that instead of using several PSRs this zone could be modeled only with one TSLR and the solution was going to be obtained using the LTIM. But because the PSRs are the main unit in the CRN it was decided that the implementation of the LTIM in the PSR solution (PSR-LTIM) will have a more impact than the TSLR implementation. However, the implementation of the TSLR along with the LTIM is subject of future work. 


\subsection{One Dimensional Flame LTIM}

The LTIM concept was applied to solve a one dimensional freely propagating flame. The computer program has the following characteristics.

- Energy and species transport equations were solved using finite volume method.

- The time dimension was transformed to a logarithm domain which allowed the use of relatively larger time steps.

- Time integration was performed using the second order modified Euler method. (i.e. the chemical source term was evaluated at $n$ and $n+1 / 2$ ).

- Energy and species transport equation were solved using the operator splitting technique. The transport part of the equations (convection + diffusion) was solved first then the chemical source term was integrated.

- Discretization of the transport (convection + diffusion) was performed as follows; quasisecond order upwind (QSOU) method (Amsden, O'Rouke and Butler 1989) for the convection part and $2^{\text {nd }}$ order central differences for diffusion.

- The new code is flexible and can be used with any chemical reaction mechanism in CHEMKIN format; ARM9 was used for the present study.

The physical properties and chemical source terms were calculated using CHEMKIN software subroutines.

The one dimensional flame program solved the species transport equation in the conservative form. 
$\frac{\partial\left(\rho Y_{i}\right)}{\partial t}+\frac{\partial\left(\rho u Y_{i}\right)}{\partial x}=\frac{\partial}{\partial x}\left(\rho D_{i, m} \frac{\partial}{\partial x}\left(Y_{i}\right)\right)+M W_{i} \dot{\omega}_{i}$

Where $D_{i, m}$ is the diffusion coefficient of the specie $i$ in the mixture. $D_{i, m}$ is calculated using Equation 5.23.

$D_{i, m}=\frac{1-Y_{i}}{\sum_{j \neq i}^{N} \frac{X_{j}}{D_{j, i}}}$

The term $D_{i, j}$ is the binary diffusion coefficient of the specie $i$ into the specie $j$ calculated using kinetic theory. The operator splitting was done as follows.

$$
\begin{aligned}
& \frac{\rho\left(Y_{i}^{*}-Y_{i}^{n}\right)}{\Delta t}=- \text { Conv }_{i}+\text { Diff }_{i} \\
& \frac{\rho\left(Y_{i}^{n+1}-Y_{i}^{*}\right)}{\Delta t}=S_{i}
\end{aligned}
$$

Where $\operatorname{Conv}_{i}=\frac{\partial\left(\rho u Y_{i}\right)}{\partial x}$ and Diff $f_{i}=\frac{\partial}{\partial x}\left(\rho D_{i, m} \frac{\partial}{\partial x} Y_{i}\right)$. Adding Equations 5.24 and 5.25 yields the following expression.

$$
\frac{\rho\left(Y_{i}^{n+1}-Y_{i}^{n}\right)}{\Delta t}=-\operatorname{Conv}_{i}+\operatorname{Diff}_{i}+S_{i}
$$

Equation 5.26 is equivalent to 5.22. The energy equation used in this one dimensional system has the following form. 
$\rho c_{p, m}\left(\frac{\partial T}{\partial t}+u \frac{\partial T}{\partial x}\right)=+\frac{\partial}{\partial x}\left(k_{m} \frac{\partial T}{\partial x}\right)+\sum_{i=1}^{N_{\text {ouml }}} c_{p, i} \frac{\partial T}{\partial x}\left(\rho D_{i, m} \frac{\partial Y_{i}}{\partial x}\right)-\sum_{i=1}^{N_{\text {otoml }}} h_{i} M W_{i} \omega_{i}$

Where $c_{p, m}$ is the specific heat of the mixture, $k_{m}$ thermal conductivity of the mixture, $h_{i}$ enthalpy of the specie $i$.

Integration of the chemical source terms in Equations 5.26 and 5.27 was performed using the LTIM. Finally the velocity is corrected using the continuity equation given by.

$\frac{\partial \rho}{\partial t}+\frac{\partial \rho u}{\partial x}=0$

Figure 5.6 shows the flowchart of the one dimensional flame LTIM program. 


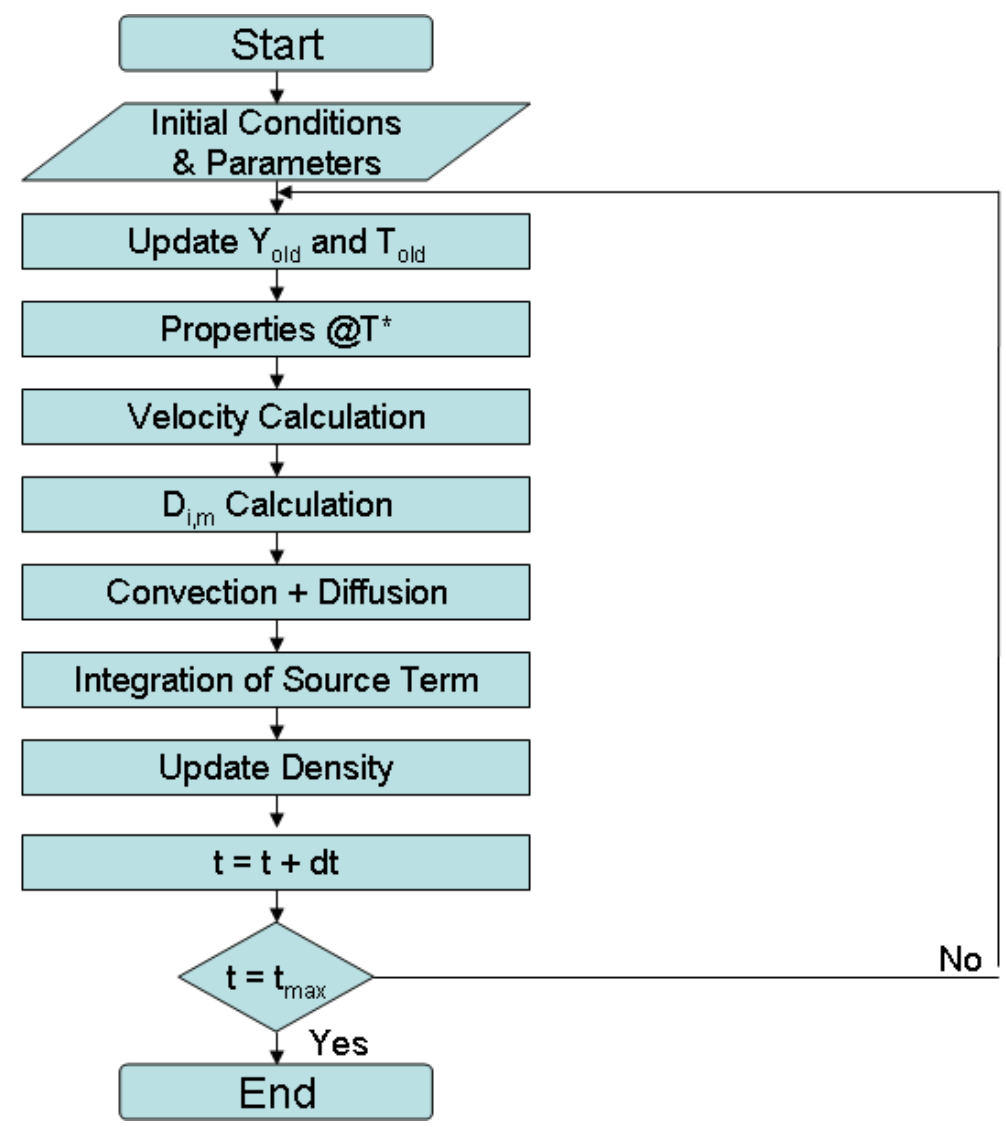

Figure 5.6. One Dimensional Flame Program Flowchart.

Figure 5.6 shows the calculation steps used in the one dimensional program. The program starts the solution reading the initial conditions supplied by the user. The transport part of the equations is solved first and then the transient source term is integrated using the LTIM. The convection and diffusion part are treated implicitly. In order to solve the implicit part the TDMA (Tri-Diagonal Matrix Algorithm) method is used.

\subsubsection{One Dimensional Flame LTIM Results at Fixed Temperature.}

In order to verify the correct implementation of the LTIM method the problem was solved for a fixed temperature problem. The solution of a methane-air flame (equivalence ratio $=0.8$ ) at atmospheric pressure with a constant temperature profile is presented next. The methane-air flame chemistry was calculated using ARM9 mechanism. 
The boundary conditions used in the species transport equation are the following.

- Dirichlet (or fixed value) boundary condition. At the inlet of the domain (west boundary).

- Neumann boundary condition, the derivative is set to zero on the exit of the domain (east boundary).

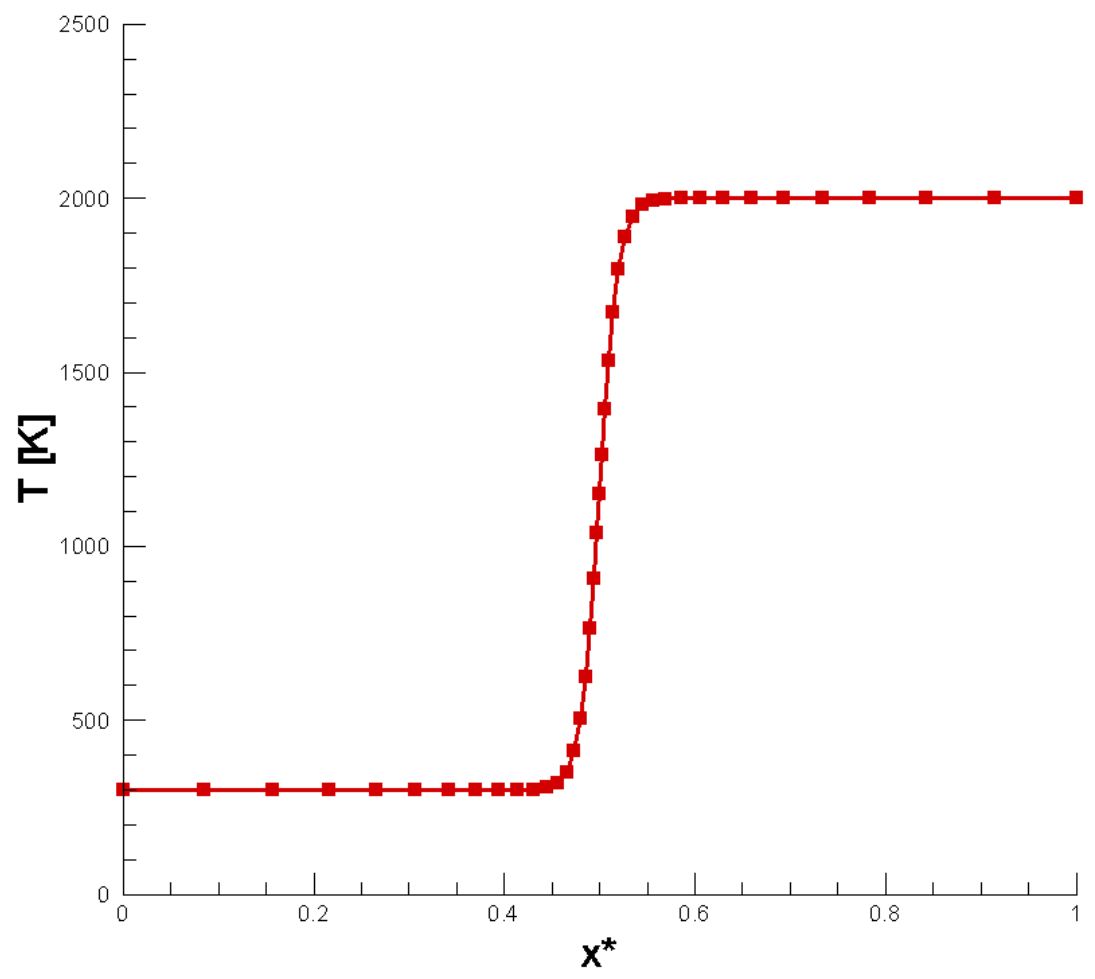

Figure 5.7. One Dimensional Flame LTIM Temperature Profile (Fixed).

The prescribed temperature profile has a similar trend to that seen in the cases where a flame is present. The fresh reactants enter from $x^{*}=0$ and travel to the right of the domain, then there is a sudden temperature increase that represents the flame region and finally the temperature remains constant at post-flame region located at the exit of the $x^{*}=1.0\left(x^{*}=\frac{x}{x_{t o t}}, x_{t o t}=5[\mathrm{~cm}]\right)$. Solution of Eq. 5.26 for the nine species present in the ARM9 mechanism is shown next. 


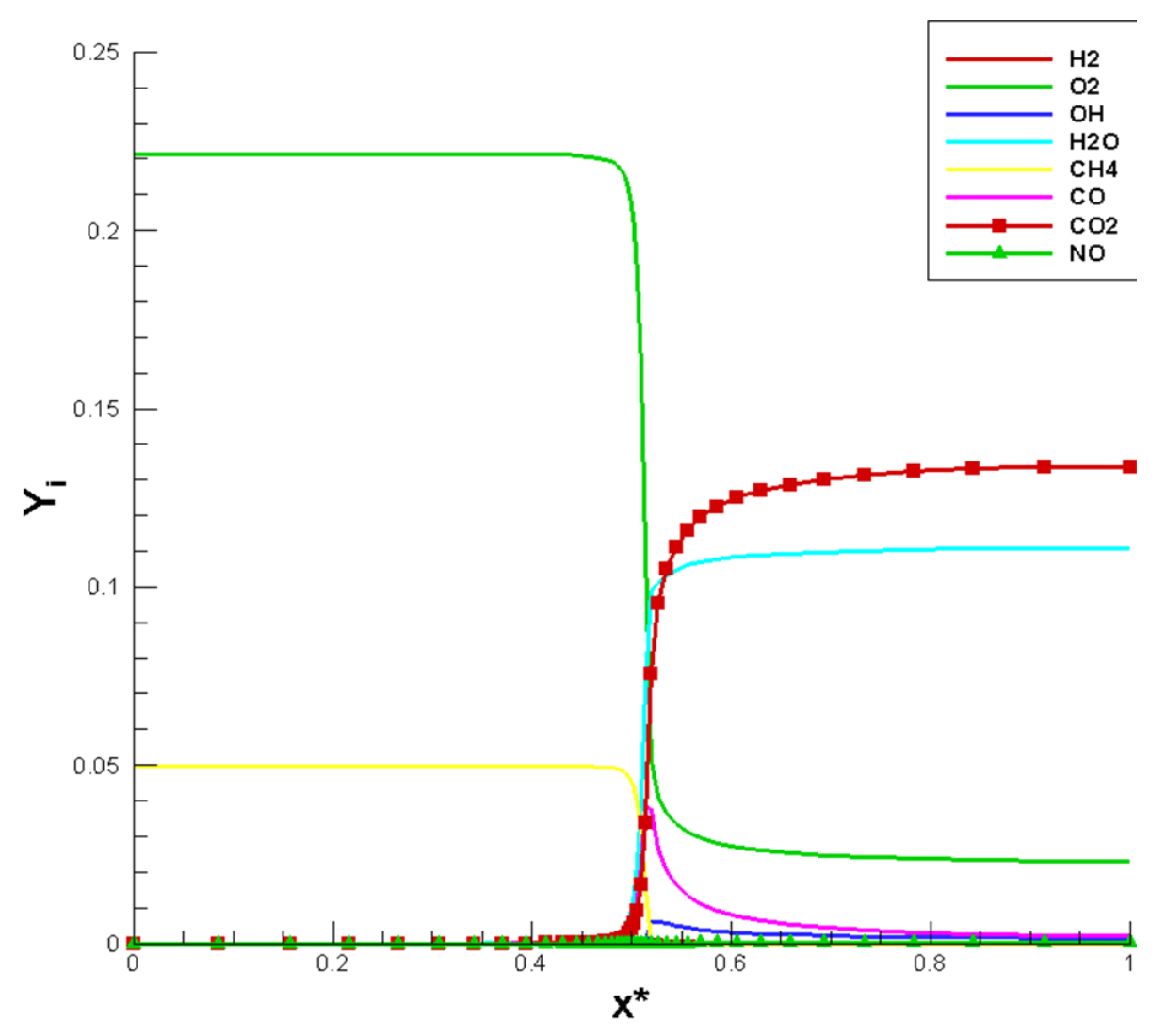

Figure 5.8. Mass Fraction Profiles at Fixed Temperature, $t=0.1[\mathrm{~s}]$.

The initial composition was set to the concentration of the system for an equivalence ratio equal to 0.8. Species profiles at $t=0.1[\mathrm{~s}]$ are shown in Figure 5.8. It was found that the species profiles did not change after $0.1[\mathrm{~s}]$. These profiles have the expected shape, due to the temperature profile shown in the Figure 5.8. Profiles were obtained using the LTIM for the integration of the chemical source term present in the transport equations. The next step was to solve the species and energy equations simultaneously.

\subsubsection{One Dimensional Flame LTIM Results at Variable Temperature.}

The LTIM is also applied to a one dimensional freely propagating methane-air flame where the chemistry is also modeled using the ARM9 mechanism. The flame prediction is for the following conditions, equivalence ratio equal to $0.9, T_{\text {in }}=300[\mathrm{~K}]$, and $P=1$ [atm]. The boundary conditions used in the simulations are: 
- Dirichlet boundary condition. At the inlet of the domain (west boundary) for temperature and species.

- Neumann boundary condition. The derivative for both species and temperature is set to zero on the exit of the domain (east boundary).

- No heat transfer in the domain. Adiabatic conditions.

Results using the LTIM (Flame-LTIM) approach were compared against the predictions obtained with CHEMKIN PREMIX software (Kee, et al. 1985) at steady state conditions.

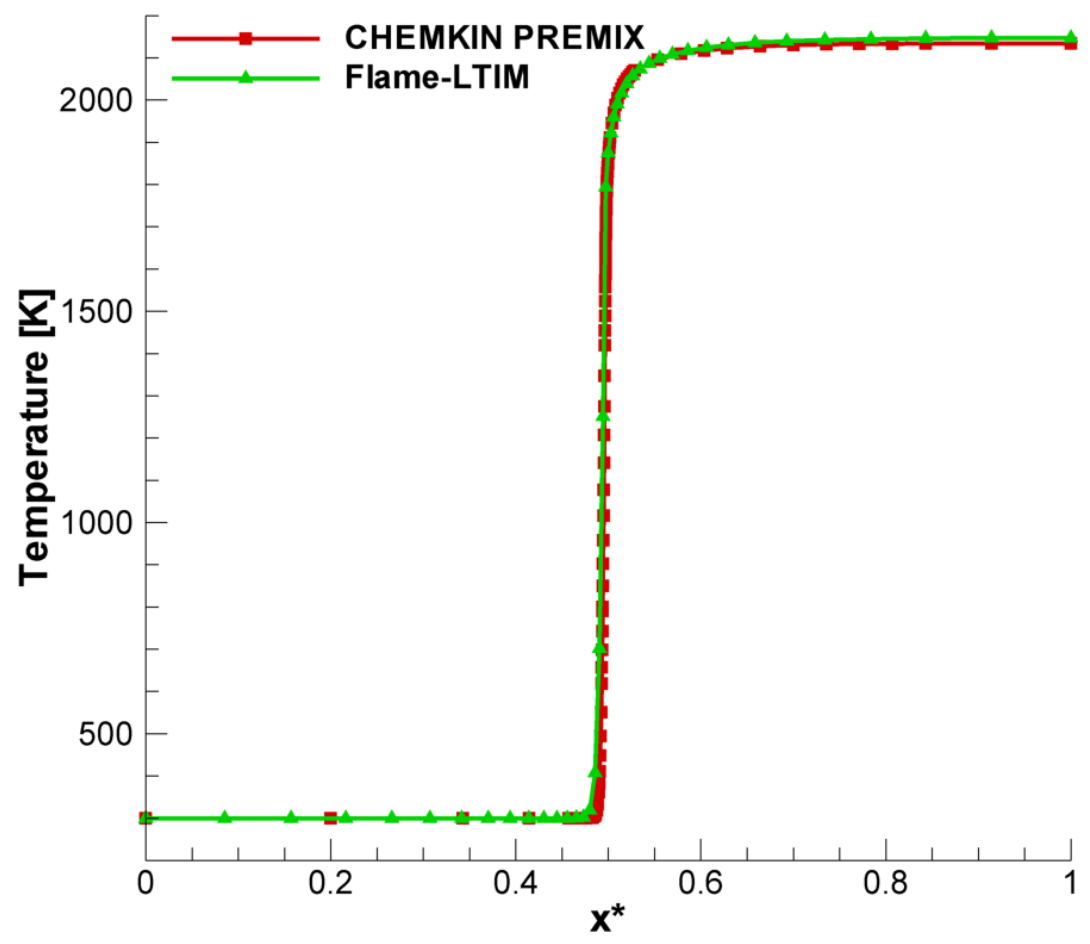

Figure 5.9. Temperature Profile Prediction.

Results obtained using the Flame-LTIM program are in good agreement with CHEMKIN PREMIX results. The adiabatic flame temperature difference between the two codes is 12 [K]. Selected species predictions are shown in Fig. 5.10. Although NO predictions for both codes 
suggest that the outflow boundary condition on the east boundary will fit better for this problem than the zero derivative boundary condition.

a)

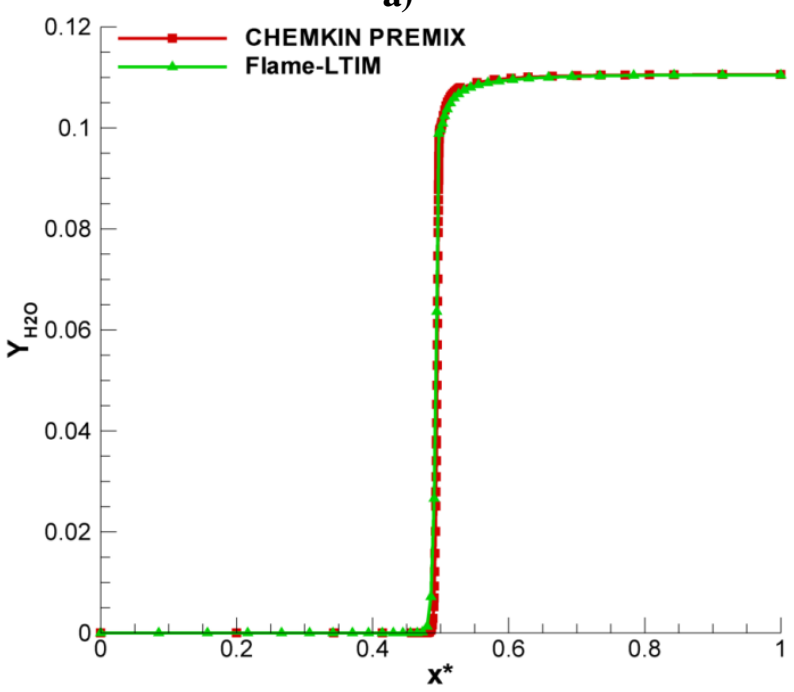

c)

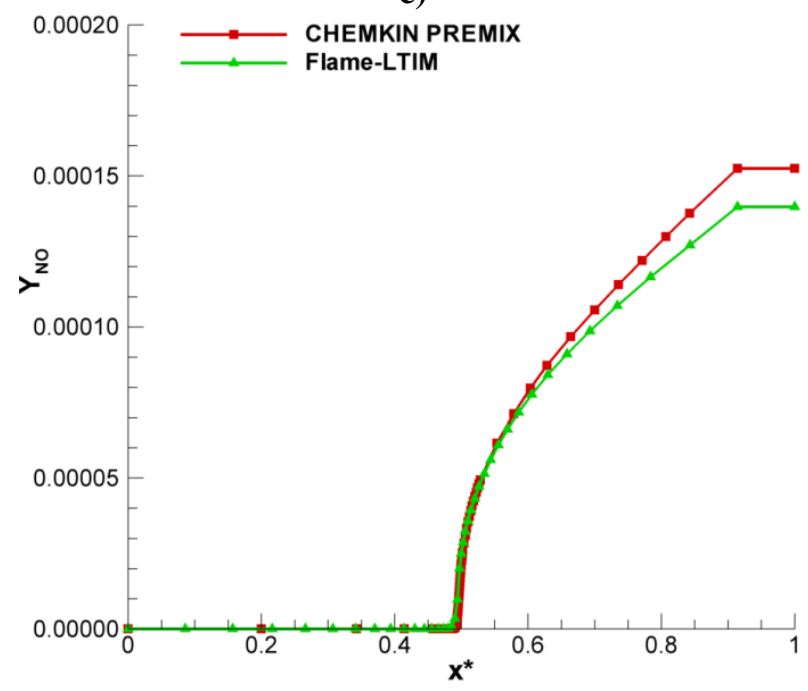

b)

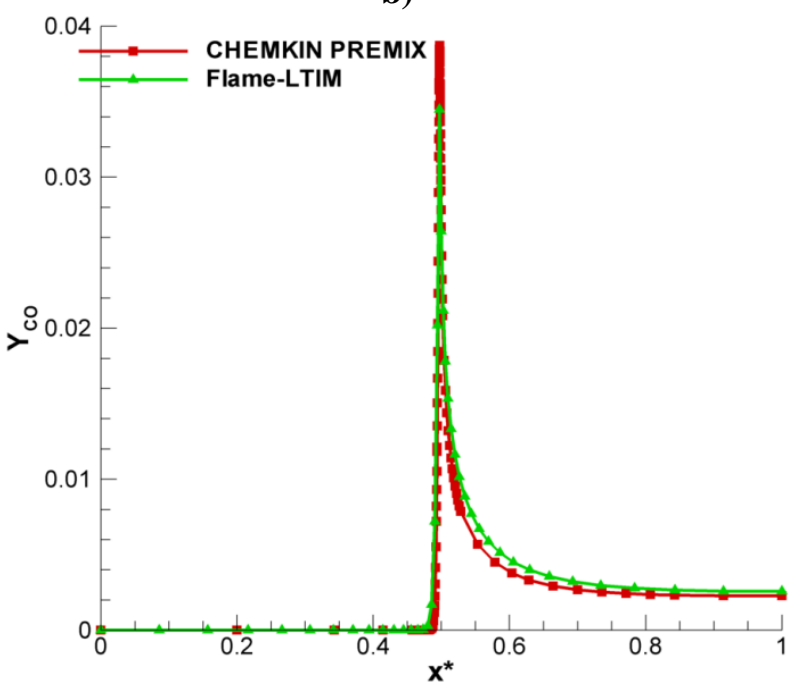

Figure 5.10. Species Mass Fraction Comparison.

The flame speed was calculated using the Flame-LTIM code by an iterative method. The method simulates the problem using an iterative scheme by probing various possible flame speed values. If the velocity proposed is larger than the flame speed the flame profile will move to the right, on the other hand if the velocity is smaller the flame will move to the left. 
Table 5.7. Flame Speed Calculation for an Equivalence Ratio $=0.9$, and $P=1$ [atm].

\begin{tabular}{|c|c|c|c|c|}
\hline $\begin{array}{c}\text { Inlet Velocity } \\
{[\mathrm{cm} / \mathrm{s}]}\end{array}$ & 40 & 31.9 & 30.6 & 30 \\
\hline $\begin{array}{c}\text { Flame } \\
\text { displacement } \\
{\left[\Delta x^{*}\right]}\end{array}$ & $2.5 \times 10^{-2}$ & $1.82 \times 10^{-2}$ & $8.5 \times 10^{-5}$ & $-8.9 \times 10^{-3}$ \\
\hline
\end{tabular}

The inlet velocity of $30.6[\mathrm{~cm} / \mathrm{s}]$ moves the flame the least for this reason this velocity value is accepted as the flame speed of the problem. The flame velocity calculated using CHEMKIN PREMIX is $30.14[\mathrm{~cm} / \mathrm{s}]$. 


\section{Chapter 6: Conclusions}

Several strategies to reduce the computational time for combustion simulations of laboratory scaled swirl stabilized burners and ideal reactors were studied. The chemical reactor network (CRN) model along with the log-time integration method (LTIM) was applied to different combustor set ups with satisfactory results.

The success of the CRN model relies on accurate flow and temperature predictions from the CFD model. It was found that the NETL burner simulations can be simplified by performing two dimensional axisymmetric simulations of the reacting flow by specifying the inlet velocity profiles obtained from the three dimensional calculations as boundary conditions. However the closest match to measurements was obtained when the velocity profiles from experiments were used as inlet boundary conditions. The predicted velocity and mass fraction profiles near the dump plane were satisfactory but they started to deviate from measurements further downstream of the dump plane. This is attributed to the inability of the turbulence model to properly capture the velocity and temperature field for this type of flow. In this regard a detailed validation study has been conducted to assess the accuracy of the CFD simulations.

It was noted that the swirl number value does not provide enough information to properly characterize the flow field in swirl combustors. The swirl number is a ratio of two integral quantities and any properly scaled velocity profile can give the same swirl number value but the predicted flow field could be very different. The difference can be appreciated in the misrepresentation of the main recirculation zone compared to experiments. It was shown that the shape of the tangential profile plays a critical role.

Numerical simulation of unconfined flames is a challenging problem due to the entrainment of the surrounding air. Simulations of the unconfined premixed swirl burner could not capture the flame shape reported in the experiments. Several simulations were performed using different 
velocity profiles and turbulence models without success. The source of this mismatch should be a topic of further study.

CRN simulations for premixed and non-premixed burners were performed using a detailed methane-air mechanism with 53 species and 325 reactions. CRN simulations of a premixed burner were compared with CFD simulations that used more complete chemical mechanisms with similar results. The simulation time needed by the CRN was considerably lower than the CFD simulations with a more complete mechanism, CRN took only one fifth of the time of CFD using ARM9 and one seventh of the time of CFD using ARM19. The concentration of the major species (mole fraction above $1 \times 10^{-3}$ ) matched closely for both CRN and CFD simulations (maximum difference was $2.8 \%$ ) for the premixed case but there was a relatively significant difference of the concentration of the minor species (e.g. for NO the difference between CRN and CFD using ARM9 was $25 \%$ and for CRN and CFD using ARM19 was 75\%). It was expected that the concentration of the species must change because the CRN predictions included more chemical species in the mechanism and the concentrations have to redistribute because the total mass of the system must be conserved.

CRN simulation of the non-premixed methane-air burner, TECFLAM, closely matched the experimental concentration of most species but overpredicted the concentration of NO (the CRN predicted an outlet NO concentration of 50 [ppm] whereas a concentration of 20 [ppm] was measured in the experiments). This was expected because the CFD simulation overpredicted the temperature (the maximum difference was $250[\mathrm{~K}]$ ). As it is stated in the literature, NO concentration significantly depends on the temperature especially above $1800[\mathrm{~K}]$ where the thermal NO pathway becomes dominant. CRN results also showed dependence on the number of reactors. The predicted NO concentration was low for the CRN with a few number of reactors $\left(n_{\text {reactors }}=26\right)$ because the temperature in each reactor is relatively low because the average 
temperature value is biased towards low temperature reactors. As the number of zones increased the NO prediction from CRN converge to a value of 50 [ppm].

The computational time of the $\mathrm{CRN}$ is a function of the number of reactors used in the network but even for large chemical reactor networks the computational time is significantly lower than the CFD simulations with more complete chemical mechanisms.

To further facilitate faster computations, various stiff solvers were considered. The present study proposed a novel integration method (LTIM) which can be applied to; closed transient reactors, perfectly stirred reactors, and one dimensional flames. The LTIM can be used as an option of the commercial software CHEMKIN.

The LTIM was successfully applied to the solution of stiff systems. The method proved to be as accurate as high order integration schemes (maximum difference was $0.6 \%$ ) while at the same time being faster (LTIM CPU is one fifth of the CPU needed by the R.K. $5^{\text {th }}$ order method).

The PSR-LTIM approach with variable time step and using equilibrium concentrations as initial condition was shown to be as accurate as the commercial PSR-CHEMKIN computer program. The computational time required by the PSR-LTIM program was found to be close to the time required by the PSR-CHEMKIN program. The CPU time needed by the PSR-LTIM was 5.3 [s] and for the PSR-CHEMKIN was 3 [s].

Finally, a one dimensional flame code was successfully solved using the LTIM for a premixed methane-air flame. Results of the Flame-LTIM matched the predicted values using CHEMKIN-PREMIX program. The temperature difference at the outlet was $0.56 \%$ and the difference in the flame velocity was $1.5 \%$. 


\subsection{Future Work}

One of the outstanding issues in combustion simulation is indeed the accurate prediction of the flow and temperature fields. RANS turbulence models are limited in this regard. Large Eddy Simulations (LES) can be performed in order to improve the prediction of the temperature and flow fields in the combustor.

CRN simulation can also be improved by modifying the reaction rates in order to include the effect of turbulent mixing. Current simulations were performed using only the finite rate approach but mixing can be incorporated using the same logic employed in the derivation of the Eddy-Dissipation concept (EDC).

The LTIM can be improved by using a totally implicit or semi-implicit scheme. In most of applications the steady state solution is what is being sought. Implicit schemes are known to allow the use of large time steps than explicit schemes but they require a special treatment of the reaction rate expressions. This is a challenging problem due the complexity of the rate expressions for detailed reaction mechanisms. 


\section{References}

Amsden, A.A., P.J. O'Rouke, and T.D. Butler. KIVA II: A Computer Program for Chemical Reactive Flows with Sprays. LA-11669-MS, Los Alamos National Laboratory, 1989.

Andreini, A. and B. Facchini. "Gas Turbines Design and Off-Design Performance Analysis with Emissions Evaluation." J. Eng. Gas Turbines Power 126, 2004: 83-91.

ANSYS-FLUENT. "Theory Guide." Canonsburg, PA, 2009.

ANSYS-FLUENT. "User's Guide." Canonsburg, PA, 2013.

Bockle, S., J. Kazenwadel, and C. Schulz. "Laser-Diagnostic Multi-Species Imaging in Strongly Swirling Natural Gas Flames." Applied Physics B, 2000.

Bragg, S.L. Aplication of Reaction Theory to Combustion Chamber Analysis. London, England: Aeronautical Research Council Pub. ARC 16170, Ministry of Defense, 1953.

Cash, J.R. and A.H. Karp. "A Variable Order Runge-Kutta Method for Initial Value Problems with Rapidly Varying Right-Hand Sides." ACM Transactions on Mathematical Software, Vol 16, 1990: 201-222.

Celik, I.B. Introductory Numerical Methods for Engineering Applications. Morgantown, WV: Ararat Books \& Publishing, 2008.

Chemical Equilibrium with Applications. February 3, 2010.

http://www.grc.nasa.gov/WWW/CEAWeb/ceaHome.htm (accessed May 22, 2014).

Chigier, N.A. and J.M. Beer. "Velocity and Static-Pressure Distributions in Swirling Air Jets Issuing from Annular and Divergent Nozzles." Transactions of the ASME, 1964: 788-796.

Escobar, J., D. Ferguson, J. Ranalli, J. Nanduri, and I. Celik. "An Experimental and Computational Study of Emissions from a Swirl Stabilized Burner under EGR Conditions." Proceeedings of the 2010 Technical Meeting of the Central States Section of the Combustion Institute. Champaign, Illinois, 2010. 
Escobar, J., I. Celik, and D. Ferguson. "Development of a Log-Time Integration Method for Reactive Flows." Proceedings of the ASME 2012 Fluids Engineering Division Summer Meeting. San Juan, P.R., 2012.

Escobar, J., J. Nanduri, I.B. Celik, and D. Ferguson. "Analysis of NOx Pathways in EGR Combustion." US National Combustion Meeting. Atlanta, GA, 2011.

Falcitelli, M., S. Pasini, and L. Tognotti. "Modelling Practical Combustion Systems and Predicting NOx Emissions with Integrated CFD Based Approach." Computers \& Chemical Engineering, Vol 26, 2002: 1171-1183.

Faravelli, T., A. Antichi, C. Callierotti, E. Ranzi, and D. Benedetto. "A Kinetic Study of an Advanced Reburning Process." Combustion Theory and Modelling, Vol 1, 1997: 377-393.

Frassoldati, A., S. Frigerio, E. Colombo, F. Inzoli, and T. Faravelli. "Determination of NOx Emissions from Strong Swirling Confined Flames with an Integrated CFD-based Procedure." Chemical Engineering Science, Vol 60, 2005: 2851-2869.

Gear, C.W. "Simultaneous Numerical Solution of Differential-Algebraic Equations." IEEE Transactions on Circuit Theory, Vol 18, 1971: 89-95.

Gicquel, L.Y.M., G. Staffelbach, and T. Poinsot. "Large Eddy Simulations of Gaseous Flames in Gas Turbine Combustion Chambers." Progress in Energy and Combustion Science Vol 38, 2012: $782-817$.

Glarborg, P., R.J. Kee, J.F. Grcar, and J.A. Miller. "PSR: A Fortran Program for Modeling WellStirred Reactors." Technical Report, SAND86-8209, Livermore, CA, 1986.

Goodwin, D., N. Malaya, H. Moffat, and R. Speth. "Cantera: An Object-Oriented Software Toolkit for Chemical Kinetics, Thermodynamics, and Transport Processes." Pasadena, CA, 2009.

Gou, X., W. Sun, Z. Chen, and Y. Ju. "A Dynamic Multi-Time Scale Method for Combustion Modeling with Detailed and Reduced Chemical Kinetic Mechanisms." Combustion and Flame, Vol 157, 2010: 1111-1121.

Grcar, J.F. "The Twopnt Program for Boundary Value Problems." Technical Report, SAND918230, Livermore, CA, 1992. 
Gupta, A.K. and A.E.E. Khalil. Private Communications, 2013.

Gustafson, J.L. "Reevaluating Amdahl's Law." Communications of the ACM Vol 31, 1988: 532533.

Hawkes, E.R., R. Sankaran, J.C. Sutherland, and J.H. Chen. "Direct Numerical Simulation of Turbulent Combustion: Fundamental Insights towards Predictive Models." Journal of Physics: Conference Series 16, 2005: 65-79.

Hindmarsh, A.C. "ODEPACK, A Systematized Collection of ODE Solvers." Technical Report, UCRL-88007, Livermore, CA, 1982.

Kanniche, M. "Coupling CFD with Chemical Reactor Network for Advanced NOx Prediction in Gas Turbine." Clean Technologies and Environmental Policy, Vol 12, 2010: 661-670.

Kee, R., J. Grcar, M. Smooke, and J. Miller. "Premix: A Fortran Program for Modeling Steady Laminar One-Dimensional Premixed Flames." Technical Report SAND85-8240, Livermore, CA, 1985.

Kee, R., R. Rupley, and J. Miller. "Chemkin III. A Fortran Chemical Kinetics Package for the Analysis of Gas-Phase Chemical and Plasma Kinetics." Technical Report SAND96-8216, Livermore, CA, 1996.

Kee, R., R. Rupley, J. Miller, M. Coltrin, J, Grcar, and E. Meeks. "Chemkin-PRO Reaction Design." San Diego, CA, 2008.

Kiehne, T.M., R.D. Matthews, and D.E. Wilson. "An Eight-Step Kinetics Mechanism for High Temperature Propane Flames." Combustion Science and Technology, Vol 54, 1987: 1-23.

Kim, H.S., A.K. Arghode, and A.K. Gupta. "Flame Characteristics of Hydrogen-Enriched MethaneAir Premixed Swirling Flames." International Journal of Hydrogen Energy, Vol 34, 2009: 1063-1073.

Landenfeld, T., A. Kremer, E.P. Hassel, J. Janicka, T. Schafer, J. Kazenwadel, C. Schulz, J. Wolfrum, "Laser-Diagnostic and Numerical Study of Strongly Swirling Natural Gas Flames." 27th Symposium (International) on Combustion, 1998. 
Launder, B.E. and D.B. Spalding. Lectures in Mathematical Models of Turbulence. New York, NY: Academic Press, 1972.

Levenspiel, O. Chemical Reaction Engineering: An Introduction to the Design of Chemical Reactors. New York, NY: John Wiley and Sons, 1962.

Lilley, D.G. "Swirl Flows in Combustion: A Review." AIAA Journal, Vol 15, 1977: 1063-1078.

Longwell, J. and M. Weiss. "High Temperature Reaction Rates in Hydrocarbon Combustion." Industrial \& Engineering Chemistry, Vol 47, 1955: 1634-1643.

Lu, T. and C.K. Law. "Diffusion Coefficient Reduction through Species Bundling." Combustion and Flame, Vol 148, 2007: 117-126.

Lu, T., C.K. Law, C.S. Yoo, and J.H. Chen. "Dynamic Stiffness Removal for Direct Numerical Simulations." Combustion and Flame, Vol 156, Issue 8, 2009: 1542-1551.

Magnussen, B.F. "On the Structure of Turbulence and a Generalized Eddy Dissipation Concept for Chemical Reaction in Turbulent Flow." 19th American Institute of Aeronautics and Astronautics Aerospace Science Meeting. St. Louis, Missouri, USA, 1981.

Magnussen, B.F. and B.H. Hjertager. "On Mathematical Models of Turbulent Combustion with Special Emphasis on Soot Formation and Combustion." Symposium (International) on Combustion Vol 16, 1977: 719-729.

Mallampalli, H.P., J.Y. Chen, and T.H. Fletcher. "Evaluation of CH4/NOx Reduced Mechanisms used for Modeling Lean Premixed Turbulent Combustion of Natural Gas." Journal of Engineering for Gas Turbines and Power, Vol 120, 1998: 703-712.

Meier, W., O. Keck, B. Noll, O. Kunz, and W. Stricker. "Investigations in the TECFLAM Swirling Diffusion Flame: Laser Raman Measurements and CFD Calculations." Applied Physics B. Vol 71, 2000: 725-731.

Momentive. Thermal Properties of Fused Quartz. http://www.momentive.com/Products/Main.aspx?id=20352 (accessed Jul 28, 2014). 
Niksa, S. and G.S. Liu. "Incorporating Detailed Reaction Mechanisms into Simulations of CoalNitrogen Conversion in P.F. Flames." Fuel, Vol 81, 2002: 2371-2385.

Novosselov, I. Chemical Reactor Networks for Combustion Systems Modeling. PhD Thesis, University of Washington, 2006.

Odgers, J. "Current Theories of Combustion within Gas Turbine Chambers." Symposium (International) on Combustion, Vol 15, 1975: 1321-1338.

Oran, E. and J. Boris. Numerical Simulation of Reactive Flow. Cambridge, UK: Cambridge University Press, 2001.

Orszag, S.A., V. Yakhot, W.S. Flannery, F., Choudhury, D. Boysan, J. Maruzewski, and B. Patel. "Renormalization Group Modeling and Turbulence Simulations." Near-Wall Turbulent Flows, 1993: 1031-1046.

Pope, S.B. "Computationally Efficient Implementation of Combustion Chemistry using In Situ Adaptive Tabulation." Combustion Theory and Modelling, 1997: 41-63.

Rubins, P.M. and D.T. Pratt. "Zone Combustion Model Development and Use: Applications to Emissions Control." American Society of Mechanical Engineers, 91-JPGC-FACT-25, 1991.

Schmittel, P., B. Gunther, B. Lenze, W. Leuckel, and H. Bockhorn. "Turbulent Swirling Flames: Experimental Investigation of the Flow Field and Formation of Nitrogen Oxide." Proceedings of the Combustion Institute, Vol 28, 2000: 303-309.

Smith, Gregory P., David M. Golden, Michael Frenklach, and Nigel W. Moriarty. GRI 3.0 Mech. 1999. http://www.me.berkeley.edu/gri_mech/ (accessed May 6, 2014).

Strakey, P. Private Communications, 2010.

Sung, C.J., C.K. Law, and J.Y. Chen. "Augmented Reduced Mechanisms for NO Emission in Methane Oxidation." Combustion and Flame, Vol 125, 2001: 906-919.

Swithenbank, J., I. Poll, and M.W. Vincent. "Combustion Design Fundamentals, Vol 14." Symposium (International) on Combustion, 1973: 627-638. 
Syred, N. and M. Beer. "Combustion in Swirling Flows: A Review." Combustion and Flame Vol 23, 1974: 143-201.

Turns, S.R. An Introduction to Combustion: Concepts and Applications. New York, NY: McGrawHill, 1996.

UCSD-mech. "Chemical-Kinetic Mechanism for Combustion Applications", San Diego Mechanism Webpage, Mechanical and Aerospace Engineering (Combustion Research), UCSD. 2012. http://combustion.ucsd.edu (accessed May 13, 2014).

Valera-Medina, A., N. Syred, P. Bowen, and A. Crayford. "Studies of Swirl Burner Characteristics, Flame Lengths and Relative Pressure Amplitudes." Journal of Fluids Engineering, Vol 133, 2011: 1-11.

Versteeg, H.K. and W. Malalasekera. An Introduction to Computational Fluid Dynamics: The Finite Volume Method. Essex, England: Pearson Education Limited, 2007.

Westbrook, C.K. and F.L. Dryer. "Simplified Reaction Mechanisms for the Oxidation of Hydrocarbon Fuels in Flames." Combustion Science and Technology, Vol 27, 1981: 31-43. 


\section{Appendix 1: Chemical Reaction Mechanisms}

\section{One step global mechanism}

The rate data for the one step global mechanism was obtained from a previously reported study by Westbrook and Dryer (Westbrook and Dryer 1981). The chemical reaction is defined in Equation

\section{A.1.1}

$\mathrm{CH}_{4}+2 \mathrm{O}_{2} \rightarrow \mathrm{CO}_{2}+2 \mathrm{H}_{2} \mathrm{O}$

The reaction rate of the one step global mechanism is defined as it is shown in Equation A.1.2

$k=A T^{n} \exp \left(-\frac{E_{a c t}}{R T}\right)\left[C H_{4}\right]^{a}\left[O_{2}\right]^{b}$

Where $A=2.119 \times 10^{11}, n=0, E_{a c t}=2.027 \times 10^{5}, a=0.2$, and $b=1.3$. The units are given in [kmol$\mathrm{m}-\mathrm{s}-\mathrm{kJ}-\mathrm{K}]$, and the reaction rate, $k$, units are $\left[\mathrm{kmol} / \mathrm{m}^{3}-\mathrm{s}\right]$.

\section{Two step global mechanism}

In the same fashion as the one step global mechanism the rate data was obtained from Westbrook (1981). The chemical reactions are given in Equations A.1.3 and A.1.4.

$$
\begin{aligned}
& \mathrm{CH}_{4}+\frac{3}{2} \mathrm{O}_{2} \rightarrow \mathrm{CO}+2 \mathrm{H}_{2} \mathrm{O} \\
& \mathrm{CO}+\frac{1}{2} \mathrm{O}_{2} \stackrel{f, r}{\leftrightarrow} \mathrm{CO}_{2}
\end{aligned}
$$


Where the $f$ and $r$ stand for forward and reverse, respectively. The reaction rates are defined as follows:

$k_{A .1 .3}=A T^{n} \exp \left(-\frac{E_{a c t}}{R T}\right)\left[C H_{4}\right]^{a}\left[O_{2}\right]^{b}$

$k_{A .1 .4, f}=A T^{n} \exp \left(-\frac{E_{a c t}}{R T}\right)\left[O_{2}\right]^{a}\left[H_{2} O\right]^{b}[C O]^{c}$

$k_{A .1 .4, r}=A T^{n} \exp \left(-\frac{E_{a c t}}{R T}\right)\left[C O_{2}\right]^{a}$

Where for (A.1.5) $A=5.012 \times 10^{11}, n=0, E_{a c t}=2.0 \times 10^{5}, a=0.7$, and $b=0.8$, (A.1.6) $A=$ $2.239 \times 10^{12}, n=0, E_{a c t}=1.7 \times 10^{5}, a=0.25, b=0.5$, and $c=1.0$, (A.1.7) $A=5 \times 10^{8}, n=0, E_{a c t}=$ $1.7 \times 10^{5}, a=1.0$. The units are given in $[\mathrm{kmol}-\mathrm{m}-\mathrm{s}-\mathrm{kJ}-\mathrm{K}]$, and the reaction rate, $k$, units are $\left[\mathrm{kmol} / \mathrm{m}^{3}-\mathrm{s}\right]$.

\section{ARM9 mechanism}

The five-step global mechanism with nine chemical species (ARM9) used in the present study was reported by Mallampalli et al. (Mallampalli, Chen and Fletcher 1998). The five reaction steps are shown in Equations A.1.8 to A.1.12.

$3 \mathrm{H}_{2}+\mathrm{O}_{2}+\mathrm{CO}_{2} \leftrightarrow 3 \mathrm{H}_{2} \mathrm{O}+\mathrm{CO}$

$\mathrm{H}_{2}+2 \mathrm{OH} \leftrightarrow 2 \mathrm{H}_{2} \mathrm{O}$ 
$3 \mathrm{H}_{2}+\mathrm{CO} \leftrightarrow \mathrm{H}_{2} \mathrm{O}+\mathrm{CH}_{4}$

A. 1.10

$\mathrm{H}_{2}+\mathrm{CO}_{2} \leftrightarrow \mathrm{H}_{2} \mathrm{O}+\mathrm{CO}$

A.1.11

$3 \mathrm{H}_{2}+\mathrm{CO}_{2}+2 \mathrm{NO} \leftrightarrow 3 \mathrm{H}_{2} \mathrm{O}+\mathrm{CO}+\mathrm{N}_{2}$

A.1.12

The kinetic data file in CHEMKIN format of the ARM9 mechanism can be accessed in the following website; www.et.byu.edu/ tom/Papers/Hemant-WSS96/WSS.html. The website was last visited on May 30, 2014.

\section{ARM19 mechanism}

The fifteen-step global mechanism with 19 chemical species (ARM19) used in the present study was reported by Sung et al. (Sung, Law and Chen 2001). The fifteen reaction steps are shown in Equations A.1.13 to A.1.27.

$2 \mathrm{H}+2 \mathrm{OH} \leftrightarrow 2 \mathrm{H}_{2}+\mathrm{O}_{2}$

$2 \mathrm{H} \leftrightarrow \mathrm{H}_{2}$

A.1.14

$\mathrm{H}+\mathrm{HO}_{2} \leftrightarrow \mathrm{H}_{2}+\mathrm{O}_{2}$

$\mathrm{H}+\mathrm{H}_{2} \mathrm{O}_{2} \leftrightarrow \mathrm{H}_{2}+\mathrm{HO}_{2}$ 


$$
\mathrm{OH}+\mathrm{CH}_{3} \leftrightarrow \mathrm{H}_{2}+\mathrm{CH}_{2} \mathrm{O}
$$

$$
\mathrm{H}+\mathrm{CH}_{4} \leftrightarrow \mathrm{H}_{2}+\mathrm{CH}_{3}
$$

$$
\mathrm{H}+\mathrm{OH}+\mathrm{CO} \leftrightarrow \mathrm{H}_{2}+\mathrm{CO}_{2}
$$

$$
\mathrm{CH}_{2} \mathrm{O} \leftrightarrow \mathrm{H}_{2}+\mathrm{CO}
$$

$$
\mathrm{O}_{2}+\mathrm{C}_{2} \mathrm{H}_{2} \leftrightarrow \mathrm{H}_{2}+2 \mathrm{CO}
$$

$\mathrm{OH}+\mathrm{C}_{2} \mathrm{H}_{4} \leftrightarrow \mathrm{H}_{2}+\mathrm{CH}_{3}+\mathrm{CO}$

$\mathrm{C}_{2} \mathrm{H}_{6} \leftrightarrow \mathrm{H}_{2}+\mathrm{C}_{2} \mathrm{H}_{4}$

$\mathrm{H}+\mathrm{OH} \leftrightarrow \mathrm{H}_{2} \mathrm{O}$

$2 N O \leftrightarrow O_{2}+N_{2}$

$\mathrm{H}_{2}+\mathrm{CO}+\mathrm{NO} \leftrightarrow \mathrm{H}+\mathrm{O}_{2}+\mathrm{HCN}$

$$
3 \mathrm{H}+\mathrm{H}_{2} \mathrm{O}+\mathrm{NH}_{3} \leftrightarrow 4 \mathrm{H}_{2}+\mathrm{NO}
$$

The kinetics of the ARM19 mechanism are reported in (Sung, Law and Chen 2001). 


\section{Appendix 2: Fifth Order Runge-Kutta Integration Method}

The fifth order Runge-Kutta method was originally developed by Cash (Cash and Karp 1990), the method was developed for the solution of ordinary differential equations (ODE). The R.K. $5^{\text {th }}$ order method is an explicit integration method hence is not necessary to calculate the Jacobian matrix in order to solve the system of equations at every integration step. Because of the explicitness of the method is necessary to use small time steps in order to maintain the stability of the solution.

In order to describe the method the following ODE is used.

$y^{\prime}=\frac{d y}{d t}=f(t, y)$

Equation A.2.1 is to be integrated between $t_{n}$ and $t_{n+1}$ where the time step is $h$. In order to perform the integration is necessary to calculate the slope of Eq. A.2.1 six times as it is shown next.

$k_{1}=f\left(t_{n}, y_{n}\right)$

$k_{2}=f\left(t_{n}+a_{2} h, y_{n}+h\left[b_{21} k_{1}\right]\right)$

$k_{3}=f\left(t_{n}+a_{3} h, y_{n}+h\left[b_{31} k_{1}+b_{32} k_{2}\right]\right)$

$k_{4}=f\left(t_{n}+a_{4} h, y_{n}+h\left[b_{41} k_{1}+b_{42} k_{2}+b_{43} k_{3}\right]\right)$

$k_{5}=f\left(t_{n}+a_{5} h, y_{n}+h\left[b_{51} k_{1}+b_{52} k_{2}+b_{53} k_{3}+b_{54} k_{4}\right]\right)$ 
$k_{6}=f\left(t_{n}+a_{6} h, y_{n}+h\left[b_{61} k_{1}+b_{62} k_{2}+b_{63} k_{3}+b_{64} k_{4}+b_{65} k_{5}\right]\right)$

$y_{n+1}=y_{n}+h\left[c_{1} k_{1}+c_{2} k_{2}+c_{3} k_{3}+c_{4} k_{4}+c_{5} k_{5}+c_{6} k_{6}\right]$

Where $y_{n+1}$ is the predicted value of the function at the new time level. The values of the $a, b$, and $c$ constants are given next.
$a_{2}=\frac{1}{5}$
$a_{3}=\frac{3}{10}$
$a_{4}=\frac{3}{5}$
$a_{5}=1$
$a_{6}=\frac{7}{8}$
$b_{21}=\frac{1}{5}$
$b_{31}=\frac{3}{40}$
$b_{32}=\frac{9}{40}$
$b_{41}=\frac{3}{10}$
$b_{42}=-\frac{9}{10}$
$b_{43}=\frac{6}{5}$
$b_{51}=-\frac{11}{54}$
$b_{52}=\frac{5}{2}$
$b_{53}=-\frac{70}{27}$
$b_{54}=\frac{35}{27}$
$b_{61}=\frac{1631}{55296}$
$b_{62}=\frac{175}{512}$
$b_{63}=\frac{575}{13824}$
$b_{64}=\frac{44275}{110592}$
$b_{65}=\frac{253}{4096}$
$c_{1}=\frac{37}{378}$
$c_{2}=0$
$c_{3}=\frac{250}{621}$
$c_{4}=\frac{125}{594}$
$c_{5}=0$
$c_{1}=\frac{512}{1771}$ 


\section{Appendix 3: $k$-epsilon $R N G$ and $R S M$ Turbulence Model Comparison}

It was of interest to compare the CFD simulation results using the k-epsilon RNG with the RSM turbulence model. A simulation of the unconfined premixed burner (described in Section 3.4) was performed using the RSM turbulence model. The numerical prediction was calculated using the inlet velocity profiles from experiments (Gupta and Khalil 2013), Figure $3.31 \mathrm{c}$ ). The details of the simulations are listed in Table A.3.1.

Table A.3.1. Effect of the Turbulence Model - Simulation Details.

\begin{tabular}{|c|c|}
\hline & Reacting Flow Simulation \\
\hline Computational Domain & Two Dimensional Axisymmetric \\
\hline Time Formulation & Steady State \\
\hline Pressure-Velocity Coupling & SIMPLE \\
\hline Pressure Discretization Scheme & PRESTO \\
\hline Convective Term Discretization Scheme & $1^{\text {st }}$ Order Upwind \\
\hline Turbulence Model & a) $k$-epsilon $R N G$ \\
& b) $R S M$ \\
\hline Chemical Mechanism & a) One Step Global Mechanism \\
\hline Turbulence Chemistry Interaction Model & Eddy-Dissipation Concept (EDC) \\
\hline Chemical Species Integration & ISAT \\
\hline
\end{tabular}

Numerical simulations had the same operating operation conditions that were listed in Section 3.4 and the geometry used was shown in Figure 3.28 a) along with the boundary conditions listed in Figure 3.29 a). 
a)

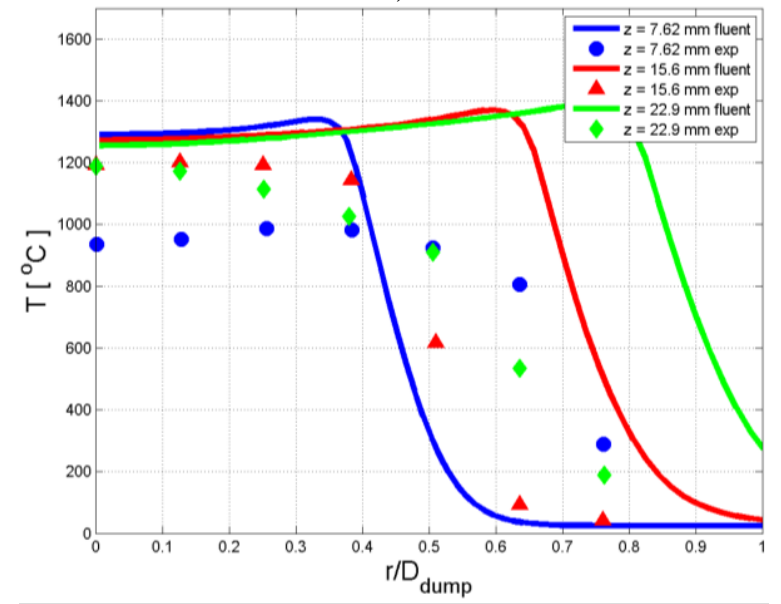

b)

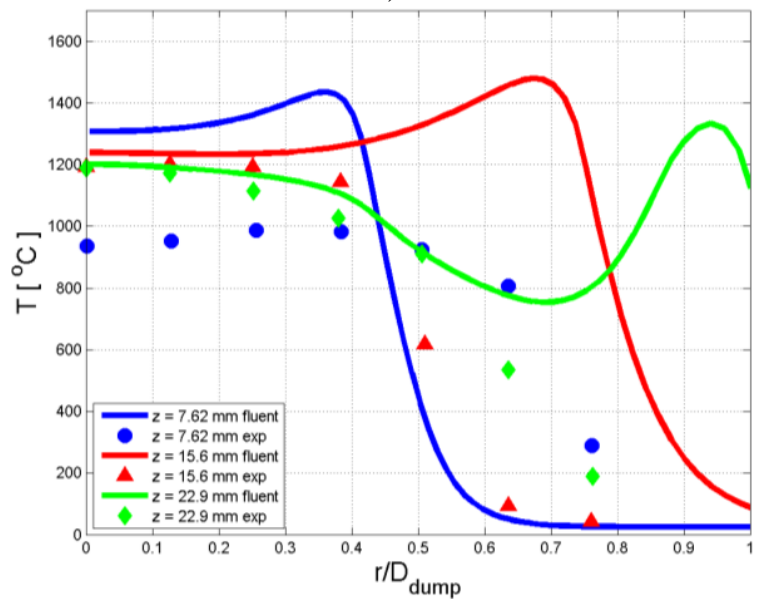

Figure A.3.1. Temperature Profile Comparison, a) k-epsilon $R N G$, b) RSM Turbulence Model.

Figure A.3.1 shows the temperature profile comparison between the k-epsilon $R N G$ and the $R S M$ turbulence model. Predicted results using RSM turbulence model overpredicted the temperature near the axis $\left(r / D_{\text {dump }}=0\right)$ by a $\Delta T=372\left[{ }^{\circ} \mathrm{C}\right]$ compared to a $\Delta T=356\left[{ }^{\circ} \mathrm{C}\right]$ calculated from the $k$ epsilon $R N G$ for the first station downstream the dumplane $(z=7.62[\mathrm{~mm}])$. But the $R S M$ prediction was better for the station located at $z=15.6[\mathrm{~mm}] \Delta T=12\left[{ }^{\circ} \mathrm{C}\right]$ compared to $\Delta T=84\left[{ }^{\circ} \mathrm{C}\right]$ from the k-epsilon $R N G$. In the same fashion the difference in the third station $z=22.9$ [mm] was close to the experiments when the $R S M$ model was used $\Delta T=45\left[{ }^{\circ} \mathrm{C}\right]$ compared to $\Delta T=62\left[{ }^{\circ} \mathrm{C}\right]$. However the temperature difference from the $R S M$ increases as the position moves in the radial direction $\left(r / D_{\text {dump }}=1\right)$. The predicted species concentrations showed no significant improvement when compared to the k-epsilon RNG turbulence model as it can be seen in Figures A.3.2 and A.3.3. 
a)

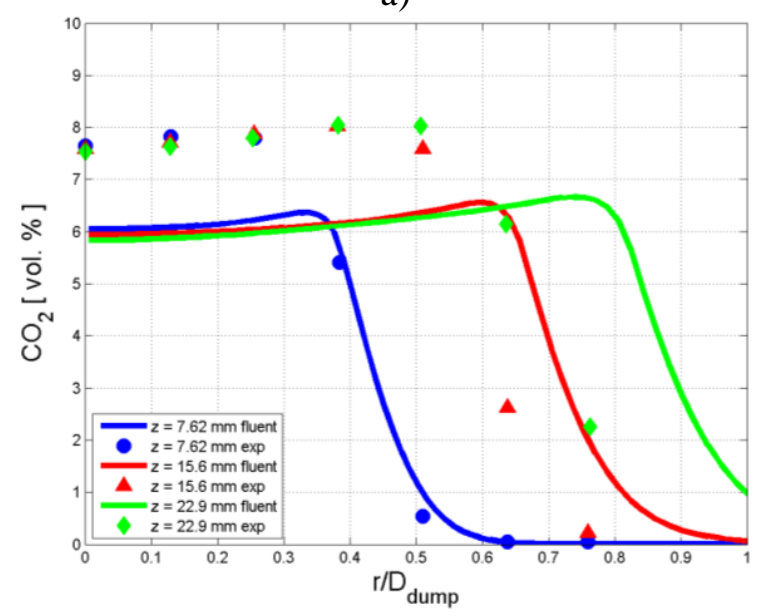

b)

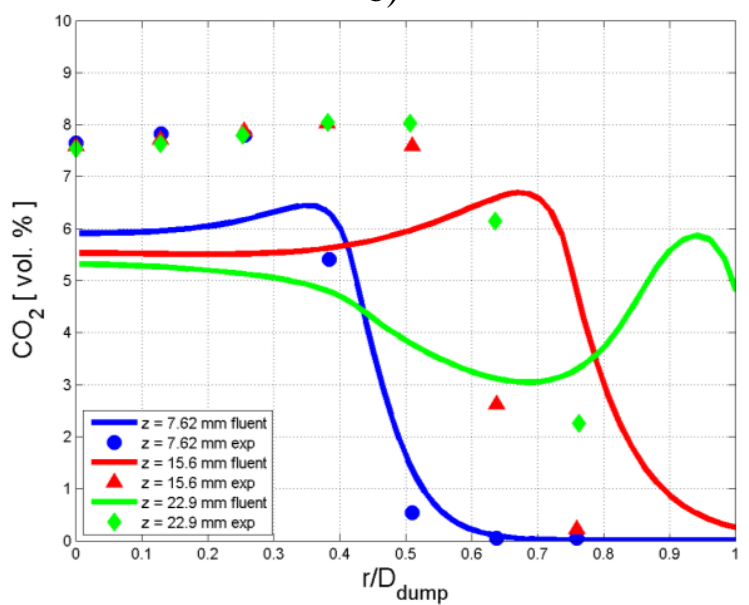

Figure A.3.2. \% Vol Carbon Dioxide Profile Comparison, a) k-epsilon RNG, b) RSM Turbulence Model.

a)

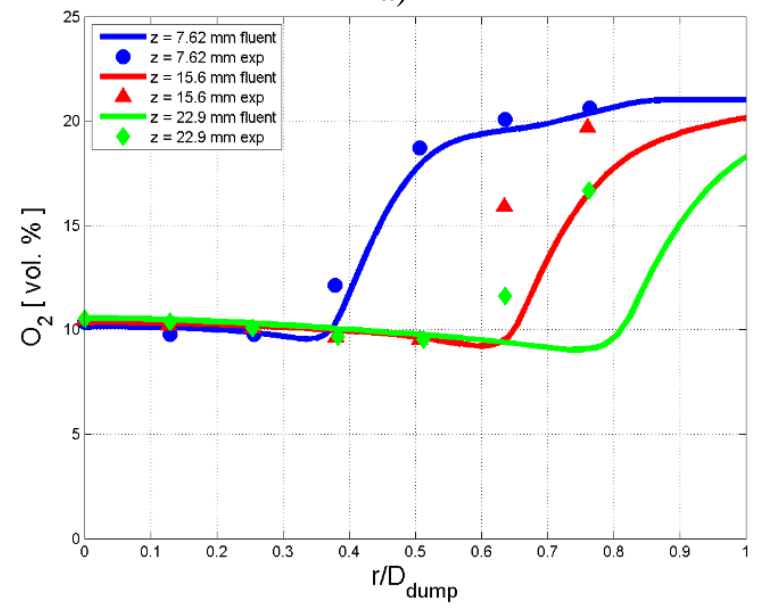

b)

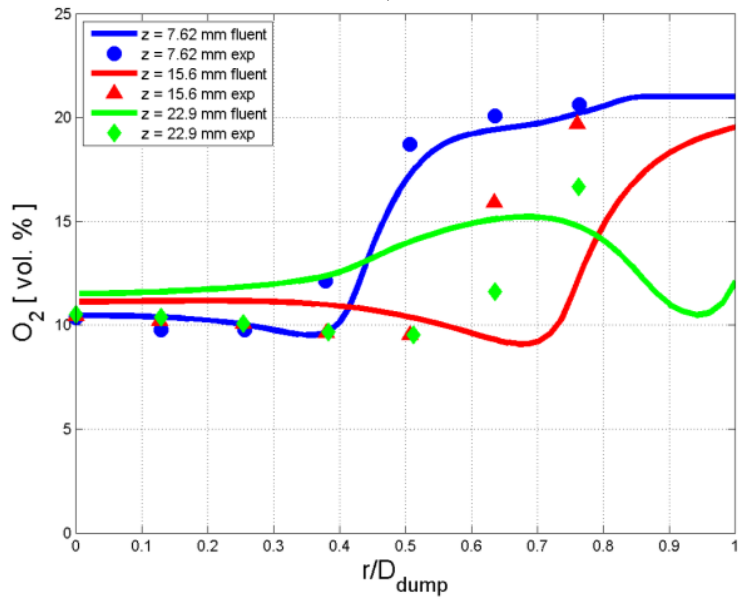

Figure A.3.3. \% Vol Oxygen Profile Comparison, a) k-epsilon RNG, b) RSM Turbulence Model.

The predicted species profiles shown in Figures A.3.2 and A.3.3 did not show a significant improvement when the RSM turbulence model was employed. The prediction using the k-epsilon $R N G$ turbulence behavior showed a better agreement with the experiments at locations near to $r / D_{\text {dump }}=1$. 